\title{
ISOLATOR-FREE DFB LASER FOR ANALOG CATV APPLICATIONS
}

\author{
By \\ Ayman Mokhtar,B.Sc., M.Sc., \\ A Thesis Submitted to \\ The Faculty of Graduate Studies and Research \\ in partial fulfillment of the requirements for the degree of \\ Doctor of Philosophy \\ Ottawa-Carleton Institute for Electrical and Computer Engineering \\ Faculty of Engineering \\ Department of Systems and Computer Engineering \\ Carleton University \\ Ottawa, Ontario, Canada
}

January, 2007

(C) 2007 Ayman Mokhtar 


$\begin{array}{ll}\begin{array}{l}\text { Library and } \\ \text { Archives Canada }\end{array} & \begin{array}{l}\text { Bibliothèque et } \\ \text { Archives Canada }\end{array} \\ \begin{array}{l}\text { Published Heritage } \\ \text { Branch }\end{array} & \begin{array}{l}\text { Direction du } \\ \text { Patrimoine de l'édition }\end{array} \\ \begin{array}{l}\text { 395 Wellington Street } \\ \text { Ottawa ON K1A ON4 }\end{array} & \begin{array}{l}\text { 395, rue Wellington } \\ \text { Ottawa ON K1A ON4 } \\ \text { Canada }\end{array}\end{array}$

Your file Votre référence ISBN: 978-0-494-23295-8 Our file Notre référence ISBN: 978-0-494-23295-8

NOTICE:

The author has granted a nonexclusive license allowing Library and Archives Canada to reproduce, publish, archive, preserve, conserve, communicate to the public by telecommunication or on the Internet, loan, distribute and sell theses worldwide, for commercial or noncommercial purposes, in microform, paper, electronic and/or any other formats.

The author retains copyright ownership and moral rights in this thesis. Neither the thesis nor substantial extracts from it may be printed or otherwise reproduced without the author's permission.
AVIS:

L'auteur a accordé une licence non exclusive permettant à la Bibliothèque et Archives Canada de reproduire, publier, archiver, sauvegarder, conserver, transmettre au public par télécommunication ou par l'Internet, prêter, distribuer et vendre des thèses partout dans le monde, à des fins commerciales ou autres, sur support microforme, papier, électronique et/ou autres formats.

L'auteur conserve la propriété du droit d'auteur et des droits moraux qui protège cette thèse. $\mathrm{Ni}$ la thèse ni des extraits substantiels de celle-ci ne doivent être imprimés ou autrement reproduits sans son autorisation.
In compliance with the Canadian

Privacy Act some supporting forms may have been removed from this thesis.

While these forms may be included in the document page count, their removal does not represent any loss of content from the thesis.
Conformément à la loi canadienne sur la protection de la vie privée, quelques formulaires secondaires ont été enlevés de cette thèse.

Bien que ces formulaires aient inclus dans la pagination, il n'y aura aucun contenu manquant.

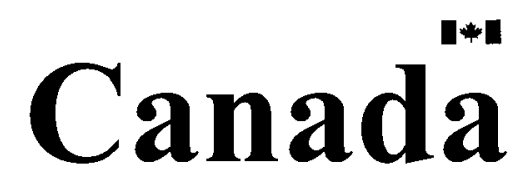




\section{Abstract}

Fiber-pigtailed Distributed Feedback (DFB) laser diode modules often employ isolators to reduce the optical feedback induced intensity noise and distortion, and to meet the requirements imposed on the DFB lasers in analog optical transmission systems such as Cable Television (CATV). For some situations however, isolators could be avoided to reduce cost and simplify laser module manufacture while maintaining the desired DFB laser's performance.

In this thesis, Fabry Perot (FP) and DFB laser diode rate equations were augmented to include the effect of optical back-reflections. The proposed laser model was implemented in software and used as the basis for linearity and noise simulations in which the effect of the reflected optical power on the laser diode performance was studied. The model's results were verified experimentally using an tunable induced back-reflection setup.

Results suggest that it is beneficial to operate unisolated DFB laser diodes in feedback Regime V. This was achieved by attaching appropriate FBG to DFB laser. Two telecommunications wavelengths, $1310 \mathrm{~nm}$ and $1550 \mathrm{~nm}$, were used to examine the performance of a DFB laser coupled to an Fiber Bragg Grating (FBG). A stable spectrum was showed and low Relative Intensity Noise (RIN) comparable to that of the isolated DFB laser was achieved for both wavelengths.

A pre-distorter model including optical back-reflection effects was derived, implemented in software, and cascaded to the laser model to compensate for the laser nonlinearity. Simulation results showed that at 0.10 modulation index, average improvements of $45 \mathrm{~dB}$ in second order harmonic distortion, $55 \mathrm{~dB}$ in third order harmonic distortion, and $40 \mathrm{~dB}$ in different intermodulation distortions can be achieved using the proposed pre-distortion technique. Furthermore, at a modulation index of 0.04 , the predistorter was found to 
reduce the second order harmonic distortion and two-tone third order intermodulation distortion levels to less than $-75 \mathrm{~dB}$ and $-100 \mathrm{~dB}$ respectively. This hence renders the laser suitable for CATV applications.

Our proposed pre-distorter constitutes a contribution to the state-of-the-art as it enhances the precision of currently used models by adding the effect of the optical feedback. 


\section{Acknowledgements}

I would like to express my deep appreciation and gratitude to Professor Samy Mahmoud, my supervisor and President of Carleton University, for his guidance and encouragement. I am greatly indebted to Professor Mahmoud for his dedication, talent, and generosity. I would also like to express my gratitude to Prof Leonard MacEachern, co-supervisor of this thesis, for his excellent advice and guidance during my doctoral research. His promptness and continual advice were highly appreciated.

I would like to express my thanks to Dr. Samy Ghoniemy for his assistance and support. I am grateful to the Department of Systems and Computer Engineering for providing a suitable research environment, and for their kind support. I would also like to recognize Prof Jacques Albert and all members of Carleton Laboratory for Induced Photonic Structures for their kind assistance. I would like to express my appreciation to Mr. Nagui Mikhail for his highly qualified assistance. I deeply thank my best friend in Ottawa, Mohamed Abou El Saoud, for his efforts.

I am grateful to my beloved country Egypt; this research would have not been possible without the financial support of the Egyptian Ministry of Defense.

I would also like to express my deep thanks to my dearest mother, her prayers and encouragement facilitate my life. I deeply thank my brothers and sisters, especially my eldest brother Wael, for all the support and encouragement.

I am indeed indebted to my dearest wife for all the sacrifices she made in order to facilitate a suitable environment for my doctoral work. I thank my daughters, MenatAllah, Yasmine, and Nour; completion of my doctorate would not have been possible without their love.

Last but never the least, thanks to my great father who passed away before I could realize this dream. 


\title{
Table of Contents
}

\begin{abstract}
$\quad$ iv
Acknowledgements $\quad$ vi

Table of Contents vii

List of Tables $\quad$ xii

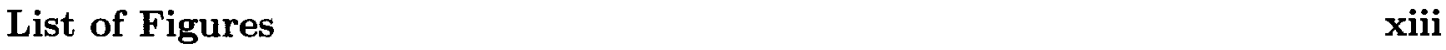

1 Introduction 1

1.1 Introduction . . . . . . . . . . . . . . . . . . 1

1.2 Thesis Motivation . . . . . . . . . . . . . . . . . . . 2

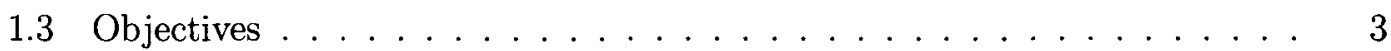

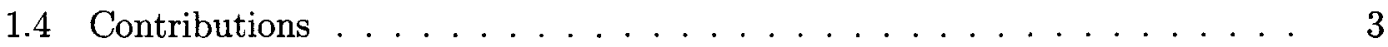

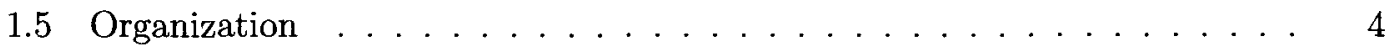

2 Background and Motivation 5

2.1 Laser Principles and Rate Equations . . . . . . . . . . . . . . . . 5

2.1.1 Laser Principles . . . . . . . . . . . . . . . . . . 5

2.1.2 Laser Diode Rate Equations Concept . . . . . . . . . . . . . . 6

2.1.3 FP vs DFB Laser Diode Rate Equations . . . . . . . . . . . . . . 7

2.2 Conventional Laser Diode Rate Equations . . . . . . . . . . . . . . 8

2.3 Modified Laser Diode Rate Equations . . . . . . . . . . . . . . . . . . . . . . . . 8

2.4 Feedback Regimes . . . . . . . . . . . . . . . . . . . . . . 10

2.5 Conventional Laser Diode Rate Equations Including Optical Feedback effect 14

2.6 Analysis of Feedback Phenomenon . . . . . . . . . . . . . . . . 16

2.7 Changes to Laser Performance due to External Cavity . . . . . . . . . . . 22

2.7.1 Emission Frequency Shift due to Feedback . . . . . . . . . . . 22

2.7.2 Single External Cavity Mode Condition . . . . . . . . . . . . . . 23

2.7.3 Spectral Linewidth Change due to Optical Feedback . . . . . . . . . 23

vii 
2.7.4 Phase and Frequency Noise of Laser with Optical Back-reflections . 23

2.7.5 Laser Intensity Noise due to Optical Feedback . . . . . . . . . . . . 24

2.7.6 Dynamic Properties of Laser with External Cavity . . . . . . . . . 25

2.8 CATV Systems . . . . . . . . . . . . . . . . . . . . . 25

2.8.1 CATV Transmitter Performance . . . . . . . . . . . . . . 28

CATV Transmitter Noise . . . . . . . . . . . . . . . . . . . . 28

CATV Transmitter Linearity . . . . . . . . . . . . . . . . . 29

Typical CATV Transmitter Performance . . . . . . . . . . . 30

2.8.2 Limiting Factors to CATV System Performance . . . . . . . . . . . 31

2.9 Motivation . . . . . . . . . . . . . . . . . . . . 32

3 Proposed Model and Simulation Results $\quad 34$

3.1 Introduction . . . . . . . . . . . . . . . . . . . . . 34

3.2 The Proposed Rate Equations . . . . . . . . . . . . . . . . . . . . . 34

3.2.1 Derivation of the Feedback Terms . . . . . . . . . . . . 35

3.2.2 Feedback Term Normalization and the Proposed Rate Equations . 38

3.3 Model Implementation . . . . . . . . . . . . . . . . . . . . . . . 39

3.3.1 Symbolically Defined Laser Diode Models Including Feedback Term 39

3.3.2 Parameters Extraction . . . . . . . . . . . . . . . . . . . 41

3.4 Simulation Results . . . . . . . . . . . . . . . . . . . . . . 41

3.4.1 Simulation of Laser Performance changes due to Feedback . . . . . 41

Possible emission frequencies . . . . . . . . . . . . . . . . . 43

Maximum oscillation frequency shift due to feedback . . . . . . . 43

Spectral linewidth change due to feedback . . . . . . . . 45

3.4 .2 DC Simulation . . . . . . . . . . . . . . . . . 47

3.4 .3 DFB Laser Diode Frequency Response . . . . . . . . . . . . . . . . 47

3.4 .4 DFB Laser Diode Distortion . . . . . . . . . . . . . . . . . 50

3.5 Laser Model Including Feedback and Noise terms . . . . . . . . . . . 55

3.5.1 Relative Intensity Noise Calculation . . . . . . . . . . . . . . . . 58

3.5 .2 Laser output . . . . . . . . . . . . . . . . . . 58

3.5.3 Modulation Frequency Response Simulation . . . . . . . . . . . . 58

3.5 .4 RIN Simulation . . . . . . . . . . . . . . . . . . 58

RIN versus bias current for fixed unintended optical back-reflection 60

RIN versus external reflectivity . . . . . . . . . . . 60

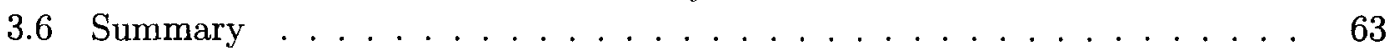

4 Experimental Work and Model Verification $\quad 64$

4.1 Introduction . . . . . . . . . . . . . . . . . . . 64

4.2 DC Measurements . . . . . . . . . . . . . . . . . . 64

4.2.1 Threshold Current Measurements Setup . . . . . . . . . . . 64

4.2.2 Threshold Current Results and Model Verification . . . . . . . . . 65

viii 
4.2.3 DFB Laser Diode Spectra Measurement Setup and Results . . . . 66

4.3 Noise and Linearity Measurements . . . . . . . . . . . . . . 70

4.3.1 RIN Measurements Experimental Setup . . . . . . . . . . . . . . 72

4.3.2 RIN Measurement Results and Model Verification . . . . . . . . . . 72

RIN versus bias current . . . . . . . . . . . . . . . 72

RIN versus external reflectivity . . . . . . . . . . 76

4.3.3 Modulation Frequency Response Measurements . . . . . . . . . . 82

4.3.4 Distortion Measurements Setup and Results . . . . . . . . . . . 83

4.4 Summary . . . . . . . . . . . . . . . . . . . 88

$5 \quad$ FBG Design and Implementation $\quad \mathbf{8 9}$

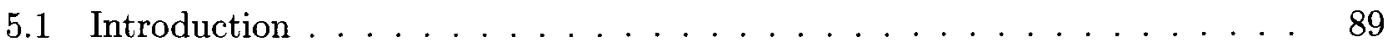

5.2 FBG Introduction and Basic Calculations . . . . . . . . . . . . . 89

$5.2 .1 \quad$ FBG Principles . . . . . . . . . . . . . . . . . . . . . . 89

5.2.2 FBG Parameter Calculations . . . . . . . . . . . . . . 91

Laser output parameters . . . . . . . . . . . . . . . . . 91

FBG parameters ...................... 91

5.3 OptiGrating Design Software . . . . . . . . . . . . . . . . . . . . . . . . . . . . . . . .

5.4 FBG Implementation . . . . . . . . . . . . . . . . . . . . . . . . 93

5.4 .1 FBG Implementation Procedures . . . . . . . . . . . . . . . . . . . 93

Optical fiber preparation . . . . . . . . . . . . . . 93

Phase mask . . . . . . . . . . . . . . . . 93

Interferometric (Holographic) technique . . . . . . . . . . . 94

Post processing of Bragg grating . . . . . . . . . . . . . . . 94

5.4 .2 The Implemented FBG Characteristics . . . . . . . . . . . . . . 94

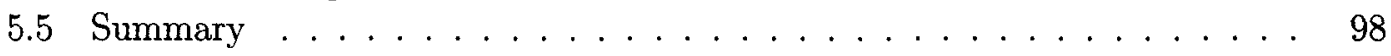

6 DFB Laser Diode with External FBG $\quad 99$

6.1 Spectrum Stability . . . . . . . . . . . . . . . . . . 100

$6.1 .11550 \mathrm{~nm}$ wavelength range . . . . . . . . . . . 100

$6.1 .21310 \mathrm{~nm}$ wavelength range . . . . . . . . . . . . . . . . . . . . . . . . . . . 102

6.2 Relative Intensity Noise Measurements . . . . . . . . . . . . . . . . 103

$6.2 .11550 \mathrm{~nm}$ wavelength range . . . . . . . . . . . . . 103

$6.2 .21310 \mathrm{~nm}$ wavelength range . . . . . . . . . . . . . . 107

6.3 Distortion Measurements . . . . . . . . . . . . . . . . . . . . 108

$6.3 .11550 \mathrm{~nm}$ wavelength range . . . . . . . . . . . . . 108

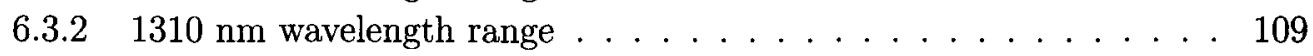

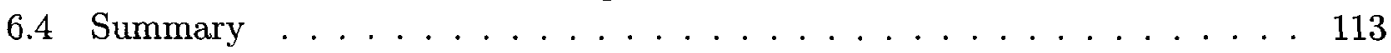

$\mathrm{ix}$ 
7 Inverse Laser Model Including Feedback Derivation and Modeling $\mathbf{1 1 4}$

7.1 Introduction . . . . . . . . . . . . . . . . . . . . . . 114

7.2 Inverse Laser Model Including Feedback Derivation . . . . . . . . . . . . . 115

7.2 .1 Volterra Series. . . . . . . . . . . . . . . . . . 120

7.2 .2 Extraction of Volterra Transfer Functions . . . . . . . . . . . . 120

Harmonic-input method . . . . . . . . . . . . . . . 120

Direct expansion method . . . . . . . . . . . . . . . 121

7.3 Inverse Laser Model Including Feedback Implementation . . . . . . . . . . 124

7.4 Typical CATV system . . . . . . . . . . . . . . . . . . . . 132

7.5 Summary . . . . . . . . . . . . . . . . . . . 137

8 Conclusions and Future Work $\quad 138$

8.1 Conclusions . . . . . . . . . . . . . . . . . . . . . . . . . . 138

8.2 Future Work . . . . . . . . . . . . . . . . . . . . . . 140

Appendix A Measurements Equipments and Results $\quad 143$

A.1 Different Equipments Used in The Measurements . . . . . . . . . . . . . . 143

A.1.1 DFB laser diodes, laser mount and laser controller . . . . . . . . . . 143

A.1.2 Fiber optic beam splitter . . . . . . . . . . . . . . . . . . 143

A.1.3 Fiber optic reflector . . . . . . . . . . . . . . . . . . . . 144

A.1.4 Variable attenuator . . . . . . . . . . . . . . . . 144

A.1.5 Optical multimeter . . . . . . . . . . . . . . . . . . 145

A.1.6 Optical Spectrum Analyzer . . . . . . . . . . . . . . . 145

A.1.7 Polarization controller . . . . . . . . . . . . . . . . . . . . 147

A.2 RIN Measurements Results . . . . . . . . . . . . . . . . . . . . . . 147

$\begin{array}{lr}\text { Appendices } & 143\end{array}$

Appendix B DFB Laser Diode with External FBG 151

B.1 FBG Design Steps . . . . . . . . . . . . . . . . . . . 151

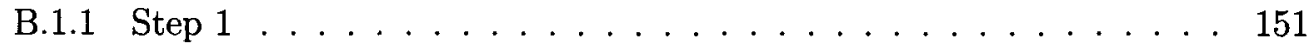

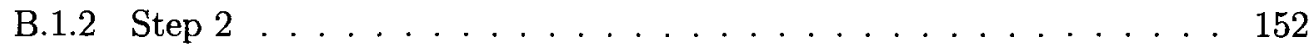

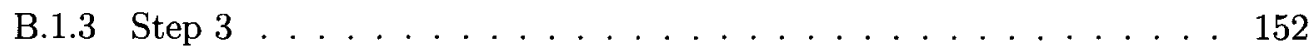

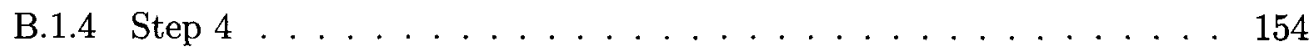

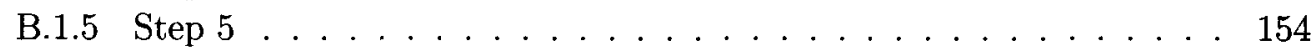

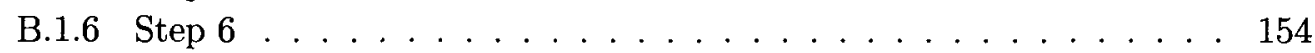

B.2 FBG Transmission and Reflection Characteristics . . . . . . . . . 156

B.3 Spectrum Stability of DFB Laser with FBG . . . . . . . . . . . . . . 163

B.4 RIN Measurements of DFB Laser with FBG . . . . . . . . . . . . . . . . 166

B.5 Distortion Measurements of DFB Laser with FBG . . . . . . . . . . 168 
Bibliography 


\section{List of Tables}

2.1 Typical CATV transmitter specifications . . . . . . . . . . . . . . 31

3.1 Laser parameters . . . . . . . . . . . . . . . . . . . . . . 42

4.1 Measured average RIN at $1 \mathrm{GHz}$ frequency offset for different bias levels . $\quad 76$

4.2 Measured average RIN at $1 \mathrm{GHz}$ frequency offset for $15 \mathrm{~mA}$ bias current and different feedback levels . . . . . . . . . . . . . . . . . . 81

6.1 Various components used in the measurements . . . . . . . . . . . . . . 99

6.2 Measured average RIN for various DFB5 configurations . . . . . . . . . 106

6.3 Measured distortion for various DFB5 configurations . . . . . . . . . 111

7.1 Various distortion components improvement . . . . . . . . . . 133 


\section{List of Figures}

2.1 Laser principle operation . . . . . . . . . . . . . . 6

2.2 Cavity loss and related effective reflectivity for DFB lasers . . . . . . 9

2.3 FeedBack regimes . . . . . . . . . . . . . . . . . . . . 12

2.4 DFB laser diode spectra under different feedback levels . . . . . . . . . . . 13

2.5 Laser cavity with external feedback . . . . . . . . . . . . . . . . 17

2.6 Round trip phase change . . . . . . . . . . . . . . . . . . 21

2.7 Calculated frequency noise spectrum . . . . . . . . . . . . . . 24

2.8 Calculated RIN for different external cavity lengths . . . . . . . . . . 26

2.9 Measured RIN for different feedback levels . . . . . . . . . . . . . . 27

2.10 Basic structure of a Hybrid Fiber/Coax CATV system . . . . . . . . . 28

3.14 -port SDD laser model with external feedback . . . . . . . . . . . . . 41

3.2 Round trip phase change versus the optical frequency . . . . . . . . . . . . 44

3.3 Maximum oscillation frequency shift versus laser cavity length . . . . . . . 44

3.4 Maximum oscillation frequency shift versus laser exit facet reflectivity . . . 45

3.5 Maximum oscillation frequency shift versus external reflectivity . . . . . 46

3.6 Spectral linewidth with feedback . . . . . . . . . . . . . . . . . 46

3.7 Swept-parameter DC simulation setup . . . . . . . . . . . . . . 48

3.8 Simulation results for threshold current change due to feedback . . . . . 49

3.9 Simulated threshold current levels at different external reflectivity . . . . . 49

3.10 Harmonic balance simulation setup for the laser model with external feedback ............................. 50 50

3.11 Modulation frequency response for the the laser diode with and without external feedback . . . . . . . . . . . . . . . . 51

xiii 
3.12 Distortion simulation setup for laser diode with and without feedback . . .

3.13 Simulated second harmonic distortion vs. external reflectivity for different bias current levels . . . . . . . . . . . . . . . . . . . 53

3.14 Simulated third harmonic distortion vs. external reflectivity for different bias current levels . . . . . . . . . . . . . . . . . . . 54

3.15 Simulated second harmonic distortion vs. bias current for different external reflectivity levels . . . . . . . . . . . . . . . . . . 54

3.16 Simulated third harmonic distortion vs. bias current for different external reflectivity levels . . . . . . . . . . . . . . . . . . 55

3.17 ADS laser model including feedback terms and noise operators . . . . . . 57

3.18 Time domain laser model's output . . . . . . . . . . . . . . . . . . 59

3.19 Simulated modulation frequency response . . . . . . . . . . . . 60

3.20 Simulated RIN . . . . . . . . . . . . . . . . . . . . . . 61

3.21 Simulated RIN versus bias current . . . . . . . . . . . . . . . . . 61

3.22 Simulated RIN versus external reflectivity $\ldots \ldots \ldots$. . . . . . . . 62

4.1 DC measurements experiment setup block diagram . . . . . . . . . . 65

4.2 Experiment setup schematic diagram for DC measurements . . . . . . . . . 66

4.3 The P-I curve for $1550 \mathrm{~nm}$ DFB laser diode under $-14 \mathrm{~dB}$ feedback ratio . $\quad 67$

4.4 Threshold current change for $1550 \mathrm{~nm}$ DFB laser diode under $-14 \mathrm{~dB}$ feedback ratio . . . . . . . . . . . . . . . . . . 67

4.5 Threshold current change for $1310 \mathrm{~nm}$ DFB laser diode under -14 $\mathrm{dB}$ feedback ratio . . . . . . . . . . . . . . . . . . . 68

4.6 Measured and simulated threshold current change due to optical back-reflection 68

4.7 DFB laser diode spectra experiment setup block diagram . . . . . . . . . 69

$4.81310 \mathrm{~nm}$ DFB laser diode spectra under different feedback powers . . . . . 70

4.9 Agilent lightwave signal analyzer . . . . . . . . . . . . . . . . 71

4.10 Relative intensity noise measurement setup . . . . . . . . . . . . . 73

4.11 Measured relative intensity noise at $25 \mathrm{~mA}$ bias current . . . . . . . . 73

4.12 Measured relative intensity noise at $30 \mathrm{~mA}$ bias current . . . . . . . . . . . 74

4.13 Measured relative intensity noise at $35 \mathrm{~mA}$ bias current . . . . . . . . . . 74

xiv 
4.14 Measured relative intensity noise at $40 \mathrm{~mA}$ bias current . . . . . . . . 75

4.15 Measured relative intensity noise at $60 \mathrm{~mA}$ bias current . . . . . . . . . 75

4.16 Measured and simulated relative intensity noise . . . . . . . . . . 77

4.17 measured relative intensity noise for bias current level of $15 \mathrm{~mA}$ and external reflectivity of $-45 \mathrm{~dB} \ldots \ldots \ldots \ldots 77$

4.18 Measured relative intensity noise for bias current level of $15 \mathrm{~mA}$ and external reflectivity of $-40 \mathrm{~dB} \ldots \ldots \ldots \ldots 78 \ldots \ldots \ldots$

4.19 Measured relative intensity noise for bias current level of $15 \mathrm{~mA}$ and external reflectivity of $-27 \mathrm{~dB} \ldots \ldots \ldots \ldots \ldots \ldots$

4.20 measured relative intensity noise for bias current level of $15 \mathrm{~mA}$ and external reflectivity of $-20 \mathrm{~dB} \ldots \ldots \ldots \ldots 79 \ldots \ldots \ldots$

4.21 Measured relative intensity noise for bias current level of $15 \mathrm{~mA}$ and external reflectivity of $-10 \mathrm{~dB} \ldots \ldots \ldots \ldots 79$

4.22 Simulated and measured RIN for different feedback powers . . . . . . . . 81

4.23 Modulation frequency response measurement setup . . . . . . . . . . 83

4.24 DFB relaxation oscillation frequency with $-45 \mathrm{~dB}$ feedback level . . . . . 84

4.25 DFB relaxation oscillation frequency with $-40 \mathrm{~dB}$ feedback level . . . . . . 84

4.26 DFB relaxation oscillation frequency with $-15 \mathrm{~dB}$ feedback level . . . . . 85

4.27 DFB relaxation oscillation frequency with $-10 \mathrm{~dB}$ feedback level . . . . . 85

4.28 Distortion measurement setup . . . . . . . . . . . . . . . 86

4.29 Measured second order harmonic distortion of DFB laser at different feedback levels . . . . . . . . . . . . . . . . . . . . 87

4.30 Measured third order harmonic distortion of DFB laser at different feedback levels . . . . . . . . . . . . . . . . . . . . 88

$5.1 \quad$ FBG structure . . . . . . . . . . . . . . . . . . . . 9 90

5.2 DFB laser diode spectrum at different bias current levels . . . . . . . . 92

5.3 Phase mask . . . . . . . . . . . . . . . . . . . . . . 93

5.4 Interferometric technique $\ldots \ldots \ldots \ldots \ldots$

$5.5 \quad$ FBG setup . . . . . . . . . . . . . . . . . . . 95

5.6 Writing FBG $\ldots \ldots \ldots \ldots \ldots$ 
5.7 The phase mask used in FBG implementation . . . . . . . . . . . . 96

5.8 FBG1 reflection and transmission characteristics . . . . . . . . . 97

6.1 Spectrum of DFB5 attached to FBG1 . . . . . . . . . . . . . . 101

6.2 Spectrum of DFB5 attached to FBG2 . . . . . . . . . . . . . . 101

6.3 Spectrum of DFB2 attached to FBG12 . . . . . . . . . . . . . . 102

6.4 Spectrum of DFB2 attached to FBG12 . . . . . . . . . . . . . 103

6.5 Measured average RIN of DFB5 . . . . . . . . . . . . . . . 104

6.6 Measured average RIN of DFB5 attached to a $30 \mathrm{~dB}$ optical isolator . . . 104

6.7 Measured average RIN of DFB5 attached to FBG10 . . . . . . . . . . 105

6.8 Measured RIN of DFB5 attached to FBG11 . . . . . . . . . . . 106

6.9 Measured RIN of DFB2 laser diode attached to FBG12 . . . . . . . . 107

6.10 Measured RIN of DFB1 _. . . . . . . . . . . . . . . 108

6.11 Measured second order harmonic distortion of DFB5 attached to FBG1 . . 109

6.12 Measured third order harmonic distortion of DFB5 attached to FBG1 . . . 110

6.13 Measured second order harmonic distortion of DFB5 attached to FBG2 . . 110

6.14 Measured third order harmonic distortion of DFB5 attached to FBG2 . . . 111

6.15 Measured second order harmonic distortion of DFB2 attached to FBG12 . 112

6.16 Measured third order harmonic distortion of DFB2 attached to FBG12 . . 113

7.1 Block diagram of the laser inverse model . . . . . . . . . . . . . . 123

7.2 ADS implementation of the laser inverse model . . . . . . . . . . . . 125

7.3 ADS setup of the laser inverse model cascaded to the laser model . . . . 126

7.4 Simulated second order harmonic with and without PD . . . . . . . . . 127

7.5 Simulated third order harmonic with and without PD . . . . . . . . . 127

7.6 Simulated second intermodulation distortion with and without PD . . . 128

7.7 Simulated third intermodulation distortion $\left(2 f_{1}+f_{2}\right)$ with and without PD 129

7.8 Simulated third intermodulation distortion $\left(2 f_{1}-f_{2}\right)$ with and without PD 130

7.9 Simulated third intermodulation distortion $\left(2 f_{2}+f_{1}\right)$ with and without PD 131

7.10 Simulated third intermodulation distortion $\left(2 f_{2}-f_{1}\right)$ with and without PD 132

7.11 Simulated second order harmonic with and without PD for 0.04 modulation $\operatorname{index} \ldots \ldots \ldots \ldots \ldots \ldots 134$

xvi 
7.12 Simulated third order harmonic with and without PD for 0.04 modulation index . . . . . . . . . . . . . . . . . 135

7.13 Simulated third intermodulation distortion $\left(2 f_{1}+f_{2}\right)$ with and without PD 135

7.14 Simulated third intermodulation distortion $\left(2 f_{1}-f_{2}\right)$ with and without PD 136

7.15 Simulated third intermodulation distortion $\left(2 f_{2}+f_{1}\right)$ with and without PD 136

7.16 Simulated third intermodulation distortion $\left(2 f_{2}-f_{1}\right)$ with and without PD 137

8.1 Block diagram of the suggested adaptive pre-distorter . . . . . . . . . . 141

A.1 OZ Optics fiber optic beam splitter . . . . . . . . . . . . . . . . . . . 144

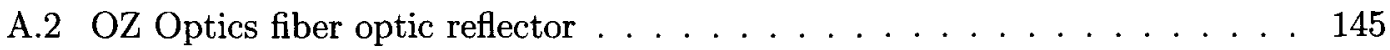

A.3 OZ Optics variable attenuator . . . . . . . . . . . . 145

A.4 Optical multimeter and laser controller . . . . . . . . . . . . 146

A.5 Agilent 86140B optical spectrum analyzer . . . . . . . . . . . . . . 146

A.6 OZ Optics polarization controller . . . . . . . . . . . . . . . . 147

A.7 Measured average relative intensity noise for bias current level of $15 \mathrm{~mA}$ and external reflectivity of $-42 \mathrm{~dB} \ldots \ldots \ldots \ldots . \ldots \ldots$

A.8 Measured average relative intensity noise for bias current level of $15 \mathrm{~mA}$ and external reflectivity of $-36 \mathrm{~dB} \ldots \ldots \ldots . \ldots 148$

A.9 Measured average relative intensity noise for bias current level of $15 \mathrm{~mA}$ and external reflectivity of $-33 \mathrm{~dB} \ldots \ldots . \ldots . \ldots . . \ldots 149$

A.10 Measured average relative intensity noise for bias current level of $15 \mathrm{~mA}$ and external reflectivity of $-29 \mathrm{~dB} \ldots \ldots \ldots \ldots$

A.11 Measured average relative intensity noise for bias current level of $15 \mathrm{~mA}$ and external reflectivity of $-24 \mathrm{~dB} \ldots \ldots \ldots \ldots$

A.12 Measured average relative intensity noise for bias current level of $15 \mathrm{~mA}$ and external reflectivity of $-15 \mathrm{~dB} \ldots \ldots \ldots \ldots$

B.1 FBG $\operatorname{design} \operatorname{step} 1 \ldots \ldots \ldots \ldots \ldots \ldots \ldots$

B.2 FBG design step $2 \ldots \ldots \ldots \ldots \ldots \ldots \ldots$

B.3 FBG design step $3 \ldots \ldots \ldots \ldots \ldots \ldots \ldots$

B.4 FBG design step $4 \ldots \ldots \ldots \ldots \ldots \ldots$

xvii 


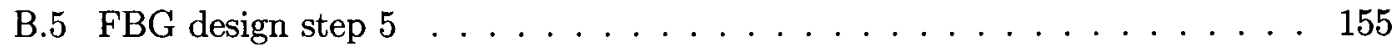

B.6 FBG design step $6 \ldots \ldots \ldots \ldots \ldots \ldots \ldots \ldots$

B.7 FBG2 reflection and transmission characteristics . . . . . . . . . . . 157

B.8 FBG4 reflection and transmission characteristics . . . . . . . . . . . 158

B.9 FBG7 reflection and transmission characteristics . . . . . . . . . . . 159

B.10 FBG10 reflection and transmission characteristics . . . . . . . . . . 160

B.11 FBG11 reflection and transmission characteristics . . . . . . . . . . . 161

B.12 FBG12 transmission spectrum . . . . . . . . . . . . . . . . 162

B.13 Spectrum of DFB5 attached to FBG4 . . . . . . . . . . . . . . 163

B.14 Spectrum of DFB5 attached to FBG7 . . . . . . . . . . . . . 164

B.15 Spectrum of DFB5 attached to FBG7 . . . . . . . . . . . . . 164

B.16 Spectrum of DFB5 attached to FBG10 . . . . . . . . . . . . 165

B.17 Spectrum of DFB5 attached to FBG11 operating at bias level of $15 \mathrm{~mA}$. . 165

B.18 Spectrum of DFB5 attached to FBG11 operating at bias level of $20 \mathrm{~mA}$. . 166

B.19 Measured RIN of DFB5 attached to FBG10 . . . . . . . . . . . . . 167

B.20 Measured RIN of DFB5 attached to FBG2 . . . . . . . . . . . . 167

B.21 Measured second order harmonic distortion of DFB5 attached to FBG4 . . 168

B.22 Measured third order harmonic distortion of DFB5 attached to FBG4 . . 169

B.23 Measured second order harmonic distortion of DFB5 attached to FBG7 . . 169

B.24 Measured third order harmonic distortion of DFB5 attached to FBG7 . . . 170

B.25 Measured second order harmonic distortion of DFB5 attached to FBG10 . 170

B.26 Measured third order harmonic distortion of DFB5 attached to FBG10 . . 171

B.27 Measured second order harmonic distortion of DFB5 attached to FBG11 . 171

B.28 Measured third order harmonic distortion of DFB5 attached to FBG11 . . 172

xviii 


\section{Chapter 1}

\section{Introduction}

\subsection{Introduction}

Fiber optic cables have many advantages over copper cables in terms of data transmission. Some of these advantages include: high bandwidth, light weight, immunity from electromagnetic radiation, and low cost [1]. Fiber optics can be used in analog optical transmission applications such as Cable Television (CATV) systems to distribute CATV signals over large geographic areas with high quality. Semiconductor laser nonlinearity and intensity noise are of concern in CATV systems. In order to provide high quality video to subscribers, CATV systems require a Carrier to Noise Ratio (CNR) of $50 \mathrm{~dB}$, Composite Second Order distortion (CSO), and Composite Triple Beat (CTB) of $-60 \mathrm{dBc}[2-4]$. $\mathrm{CSO}$ and CTB are mainly related to the different harmonic and intermodulation distortion mechanisms in the laser diode; CNR is limited by the laser's Relative Intensity Noise (RIN). One major factor increasing the RIN and distortion is optical back-reflections which can modify the static and dynamic characteristics of the laser. These reflections originate from the laser facet, laser/fiber interface, and fiber connectors.

There can be benefits to operating laser diodes with optical feedback: (i) selection of a distinct longitudinal mode of a Fabry Perot (FP) laser [5,6]; (ii) tuning of Distributed Feedback (DFB) laser emission frequency [7]; (iii) considerable linewidth narrowing [8,9]. However, optical feedback is typically expected to be detrimental to laser diode performance. For example, at some optical feedback levels laser spectral linewidth has been found to broaden by several tens of gigahertz ( $20 \mathrm{GHz}$ is equivalent to $0.16 \mathrm{~nm}$ at $\lambda=1550 \mathrm{~nm}$ ) [10]; an increase in RIN has been also observed. This large variety of both positive and negative 
effects suggests that optical feedback phenomena should be studied in detail.

Laser diode rate equations describe the interaction between the injected electrons and the generated phonons in the laser cavity. Rate equations typically neglect optical backreflections for simplicity, thereby making them ill-suited for analysis of laser diode performance with consideration of optical back-reflection effects. This work addresses the need for a comprehensive laser model with the ability to evaluate the performance changes due to optical back-reflections.

The phenomena arising in laser diodes subject to optical back reflection can be classified based on the feedback magnitude in five regimes, identified as Regimes I, II, III, IV, and $\mathrm{V}$ (ordered from least to greatest magnitude of feedback) [11]. In Regime I, the feedback level is weak and the narrowing or broadening of the laser spectral linewidth depends on the phase of the feedback. In Regime II, rapid mode hopping causes a splitting of the laser spectrum. The laser spectrum returns to a single narrow line again in Regime III. Regime IV is reached if further increase of the feedback strength is applied, the linewidth is dramatically broadened as a result. This regime is also known as coherence collapse regime. When the feedback level is increased further, the laser returns to narrow-linewidth single-mode operation and operate in Regime $\mathrm{V}$ [12].

\subsection{Thesis Motivation}

A need for a comprehensive laser model which has the ability to predict the laser diode performance due to the reflected waves (RIN, distortion, etc.) is vital. The model should also suggest the operating point based on the laser diode parameters so that transition to the coherence collapse regime can be avoided. The laser spectrum is narrowed for all feedback phases only in Regime V; the laser exhibits low RIN level and becomes insensitive to further reflections. In general this regime cannot be achieved for uncoated lasers without intentional external reflections. The challenge is to force the laser to operate in Regime V. FBG provides a good solution: including a suitable external FBG in the fiber pigtail can drive the laser diode to operate in Regime $\mathrm{V}$ and make it suitable for CATV applications. CATV systems require low distortion levels for the laser. This necessitates an effective linearization technique to compensate for laser nonlinearity. A suitable laser 
model that contains major causes of laser distortions can be a good building block for such distortion compensation technique. Further elaboration is found in the Motivation section of Chapter 2.

\subsection{Objectives}

The main objective of this thesis is to enhance the performance of the DFB laser diode for analog CATV applications and to avoid the costly isolator, thereby simplifying the laser module fabrication process while still maintaining the required laser performance. This can be achieved in the following key steps:

- Review and analyze the available semiconductor laser rate equations that include the optical back-reflections terms.

- Advance the state of the art in the area of modeling signal transmission over optical fiber links by introducing the proposed model based on the laser diode rate equations that contain the optical back-reflections terms.

- Evaluate the laser performance under optical back-reflections using the proposed models.

- Analyze the different feedback regimes.

- Avoid using the isolator needed for DFB laser diode.

- Develop a suitable technique to enhance the DFB laser diode performance in the existence of the reflected power by using an external FBG.

- Design and implement the proposed FBG.

- Evaluate laser diode performance with and without the FBG.

- Develop a suitable technique to improve the laser linearity.

\subsection{Contributions}

The main contributions in this thesis are summarized below:

- Enhancing the mathematical modeling of DFB laser diodes by including the feedback terms in the rate equations.

- Using Agilent ADS software to implement the laser model with feedback effect. 
- Prediction of the the coherence collapse regime and other feedback regimes of the DFB laser diode.

- Prediction of the DFB laser diode DC behavior, distortion, RIN, and modulation response with different back-reflection powers.

- Model verification.

- Identification of the the DFB laser diode spectrum under different feedback powers through experimentation.

- Design and implementation of the FBG needed to force the DFB laser diode to operate in Regime V.

- Performance analysis for the DFB laser diode with the FBG compared to the isolated DFB laser diode.

- Derivation and modeling of the inverse laser model including feedback to compensate for the laser diode distortion.

\subsection{Organization}

Chapter 1 introduced this work. Background and motivation to this work are presented in Chapter 2. Chapter 3 presents the model implementation and simulation results. Chapter 4 presents the experimental work and model verification. FBG design and implementation are presented in Chapter 5. In Chapter 6, the performance evaluation of DFB laser diode with an external FBG is presented and compared to that of the isolated DFB laser diode. The derivation, software implementation, and testing of the inverse laser model including optical back-reflections effect is demonstrated in Chapter 7. Finally, Chapter 8 summarizes the results obtained from this work, gives the overall conclusions, and poses recommendations for future work. 


\section{Chapter 2}

\section{Background and Motivation}

This chapter provides an explanation for the laser diode principles and the rate equation concept. The conventional laser diode rate equations and the modified gain laser diode rate equations are reviewed. The feedback phenomenon and its different regimes are analyzed, previous work that investigated feedback effects is also surveyed. Changes to laser diode performance due to optical back-reflections are discussed. Challenges to CATV systems performance are presented at the end of this chapter.

\subsection{Laser Principles and Rate Equations}

\subsubsection{Laser Principles}

LASER is an acronym for Light Amplification by the Stimulated Emission of Radiation. Ideal laser light is of a single wavelength, formed in parallel beams and it is coherent, that is, in a single phase. Laser operation involves three key light-matter interaction processes [13]:

- Photon absorbtion: when a photon of energy $\mathrm{h} \nu$ impinges on the system, an electron in state $E_{1}$ can absorb the photon energy and be excited to state $E_{2}$ as shown in Figure 2.1.a.

- Spontaneous emission: the electron at state $E_{2}$ will shortly return to the ground state without external stimulation as shown in Figure 2.1.b.

- Stimulated emission: if a photon of energy $\mathrm{h} \nu$ impinges on the system while the electron is still in its excited state, the electron is immediately stimulated to drop to the ground state and give off a photon of energy $h \nu$ in phase with the incident 
photon as shown in Figure 2.1.c.

As shown in Figure 2.1 there are two energy states, ground state $E_{1}$ and the excitedstate energy $E_{2}$. The transition between these two states involves the absorption or emission of photon of energy $E_{2}-E_{1}$. In thermal equilibrium the density of excited electrons is very small. Most photons incident on the system will therefore be absorbed, so that stimulated emission is essentially negligible. Stimulated emission will exceed absorption only if the population of the excited states is greater than that of the ground state. This condition is known as population inversion. It is achieved by various pumping techniques. In semiconductor lasers, this is accomplished by the injection of electrons into the material at the device contacts to fill the lower energy states of the conduction band.

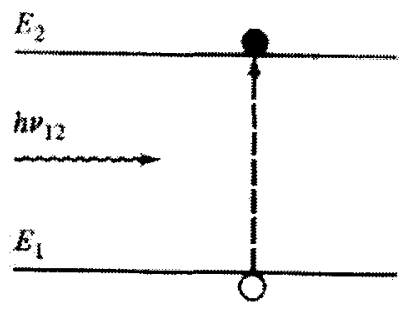

(a) Absorption

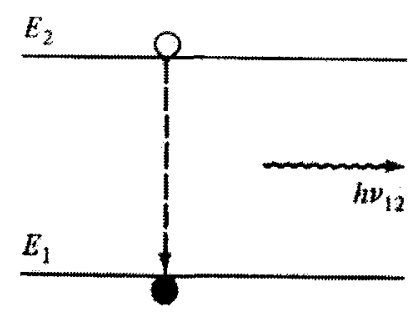

(b) Spontaneous emission

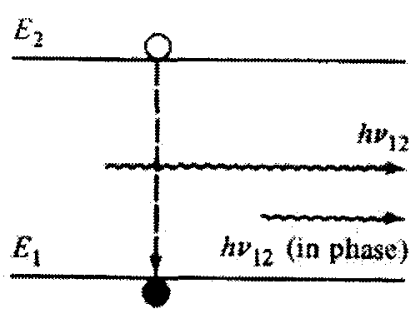

(c) Stimulated emission

Figure 2.1: The three key processes in laser operation: absorption, spontaneous emission, and stimulated emission process, reproduced from [13].

\subsubsection{Laser Diode Rate Equations Concept}

The semiconductor laser diode rate equations describe the interaction between the injected carriers and the generated photons in the laser diode active region. The total carrier population is determined by carrier injection, spontaneous recombination, and stimulated emission. For a PN junction with carrier-confinement region of depth $d$, the rate equations are given by: $\frac{d P}{d t}=$ Stimulated emission + Spontaneous emission + Photon loss; which governs the number of photons $P$, and $\frac{d C}{d t}=$ Injection + Stimulated emission + Spontaneous emission; which governs the number of electrons $C$. Under steady state condition $\frac{d P}{d t}=0$, $\frac{d C}{d t}=0$, the steady state photon density $P_{s}$ is given by: $P_{s}=$ Number of photons resulting 
from stimulated emission + Spontaneously generated photons [13].

\subsubsection{FP vs DFB Laser Diode Rate Equations}

A FP laser diode uses two end mirrors to produce multiple reflections; the lasing threshold is attained when the roundtrip gain reaches unity. The FP laser diode cavity loss $\alpha_{m}$ and the photon lifetime, $\tau_{p h}$, are given by:

$$
\begin{gathered}
\alpha_{m}=\frac{1}{2 L} \ln \left(\frac{1}{R_{1} R_{2}}\right) \\
\frac{1}{\tau_{p h}}=\nu_{g}\left(\alpha_{m}+\alpha_{i n t}\right)
\end{gathered}
$$

where $L$ is the laser cavity length, $R_{1}$ and $R_{2}$ are the two mirrors reflectivities, $\alpha_{\text {int }}$ is the internal losses, and $\nu_{g}$ is the group velocity.

A DFB laser uses a Bragg grating in the active region in order to suppress multiple longitudinal modes and enhance a single longitudinal mode, similar to the FP laser, the lasing threshold is obtained when the roundtrip gain reaches unity. The coupling coefficient $\kappa$ for a DFB laser represents the coupling between the forward and backward traveling waves due to the active region refractive index periodic change (i.e, it determines the amount of feedback due to the grating structure).

Laser diode rate equations can be used to describe DFB lasers in the same way they are used to describe FP lasers. In order to apply the photon rate equation, the photon life time $\alpha_{m}$ is required. The threshold gain of DFB depends on the coupling strength $\kappa L$. For a typical coupling strength $\kappa L=2$, Figure 2.2 on page 9 yields [10]:

$$
\alpha_{m} L \simeq 1.95
$$

for non-phase shifted DFB laser and

$$
\alpha_{m} L \simeq 1.4
$$

for $\pi / 2$-phase shifted DFB laser

The DFB cavity loss $\alpha_{m}$ can be related to an effective reflectivity $R\left(R=R_{1}=R_{2}\right)$ for a given coupling strength $\kappa L[10]$. As shown in Figure 2.2 on page 9, for a coupling 
strength of $\kappa L=2$, a DFB laser exhibits the same threshold gain as FP laser with facet reflectivities of $R=0.25$ with $\pi / 2$ phase shift, or of $R=0.14$ without phase shift.

\subsection{Conventional Laser Diode Rate Equations}

Simplified single mode rate equations were expressed in many references as follows [14-18],

$$
\begin{gathered}
\frac{d P(t)}{d t}=\Gamma G P(t)+\Gamma \beta_{s p} \frac{C(t)}{\tau_{e}}-\frac{P(t)}{\tau_{p h}} \\
\frac{d C(t)}{d t}=\frac{I(t)}{q V_{c}}-G P(t)-\frac{C(t)}{\tau_{e}}
\end{gathered}
$$

where $P(t)$ represents the intracavity photon density, $C$ is the active region carrier density, $\Gamma$ is the mode confinement factor, $\beta_{s p}$ is the spontaneous emission factor, $\tau_{e}$ is the electron life time, $\tau_{p h}$ is the photon life time, $I(t)$ is the injected current, $q$ is the electronic charge and $V_{c}$ is the volume of the active region.

\subsection{Modified Laser Diode Rate Equations}

Many works have been done by Ghoniemy et al [19] in modifying the rate equations governing the dynamics of the laser diode theory. These include, semiconductor laser gain modifications [20], the effects of device heating [21], current leakage [22] and noise [23]. Results from these work concluded the following normalized laser rate equations:

$$
\begin{gathered}
\frac{d P_{n}(T, t)}{d t}=\frac{\left(C_{n}(T, t)-C_{t r}^{n}(T)\right)}{\tau_{p h}(T)\left(1-C_{t r}^{n}(T)\right)} \frac{P_{n}(T, t)}{\sqrt{1+\bar{P}_{r}^{n}(T, t)}}-\frac{P_{n}(T, t)}{\tau_{p h}(T)} \\
+\left(\beta_{s p} B_{o} \tau_{e}(T) C_{t h o} \exp \left(\frac{T-T_{r}}{T_{o}}-\frac{E_{1} q}{K_{B} T}\right)\right) C_{n}^{2}(T, t)+F_{P_{n}}(t) \\
\frac{d C_{n}(T, t)}{d t}=\frac{I_{\text {eff }}(t)}{q V_{c} C_{t h}(T)}-\frac{\left(C_{n}(T, t)-C_{t r}^{n}(T)\right)}{\tau_{e}(T) \tau_{p h}(T)\left(1-C_{t r}^{n}(T)\right)} \frac{P_{n}(T, t)}{\sqrt{1+P_{r}^{n}(T, t)}}-\frac{C_{n}(T, t)}{\tau_{e}(T)}+F_{C_{n}}(t) \\
\frac{d \Phi_{n}(t)}{d t}=\frac{\beta}{2} G n\left(C_{n}(T, t)-\bar{C}_{n}\right)+F_{\Phi_{n}}(t)
\end{gathered}
$$




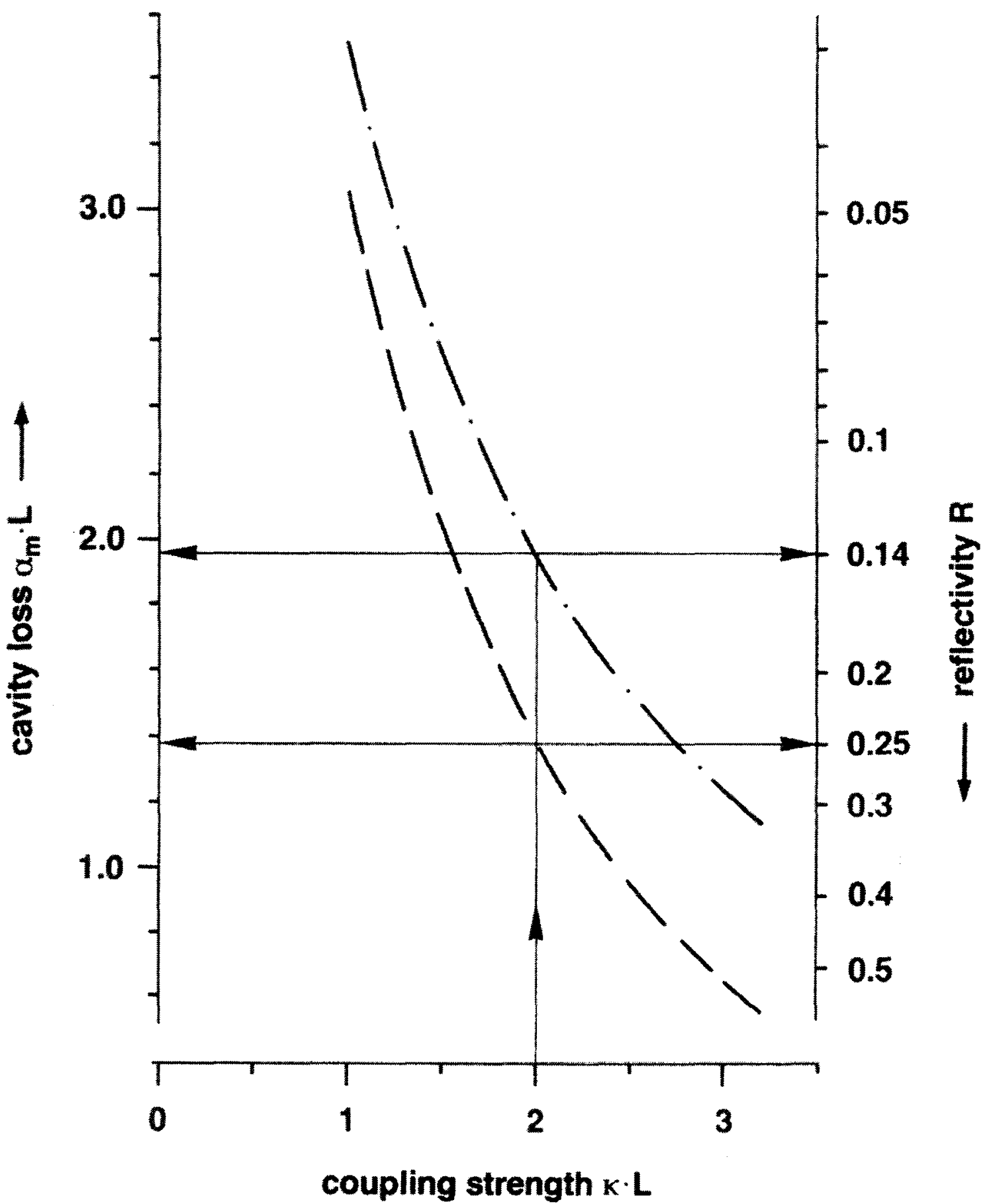

Figure 2.2: Cavity loss and related effective reflectivity for DFB lasers without phase adjustment $(-\cdot-\cdot \cdot-)$ and with a $\pi / 2$-phase shift $(-----)$, reproduced from [10]. 
where $P_{n}(T, t)$ is the normalized photon density, $C_{n}(T, t)$ is the normalized carrier density, $C_{t r}$ is the threshold carrier density, $\tau_{p h}$ is the photon life time, $\tau_{e}$ is the electron life time, $\beta_{s p}$ is the spontaneous emission factor, $T$ is the average active region temperature, $T_{o}$ is the characteristic temperature of the active region, $E_{1}$ is the activation energy of the radiative recombination, $T_{r}$ is the equilibrium temperature without injection, $K_{B}$ is the Boltzman's Constant, $B_{o}$ is the radiative recombination coefficient, $F_{P_{n}}(t)$ is the normalized photon Langevin noise sources, $I_{\text {eff }}(t)$ is the part of the external current that passes through the active region, $q$ is the electronic charge, $v_{c}$ is the volume of the active region, $C_{t h}$ is the threshold carrier density, $F_{C_{n}}(t)$ is the normalized carrier Langevin noise sources, $\Phi_{n}(t)$ is the normalized phase, $\beta$ is the linewidth enhancement factor, $\bar{C}$ is the mean of the carrier number, and $F_{\Phi_{n}}(t)$ is the normalized phase Langevin noise sources.

\section{$2.4 \quad$ Feedback Regimes}

Due to the need for coherent optical communication systems that require stable narrow linewidth sources, the effect of the optical back-reflections should be studied carefully. In [11] the laser operation in the presence of optical feedback was categorized into five regimes as follows:

- Regime I: At low feedback levels, narrowing or broadening of the laser spectrum is observed, depending on the phase of the reflected power. This was observed in [11] at a feedback level of $-80 \mathrm{~dB}$ with an external reflector at a distance of $40 \mathrm{~cm}$ from the laser facet. It is difficult to remain in this regime for long external cavity lengths. Assuming $\tau_{\text {ext }}$ of $10 \mathrm{~ns}$ the effective external reflectivity must be lower than $10^{-8}$ which is very difficult to achieve practically and the laser quite unlikely remains in Regime I.

- Regime II: Rapid mode-hopping causes a splitting in the laser emission; this splitting magnitude depends on the feedback level and the reflector location. This allows for multiple cavity modes.

- Regime III: At higher levels of feedback (approximately $-45 \mathrm{~dB}$ ), independent of the reflector distance from the laser facet, the laser is observed to operate on a single narrow line again. The range of feedback level in this region is very small, and the 
laser becomes sensitive to further reflections.

- Regime IV: Independent of the reflector distance and the feedback phase, and at a higher feedback level (approximately $-40 \mathrm{~dB}$ ), the laser linewidth broadens to as much as $50 \mathrm{GHz}$. This regime is also called coherence collapse regime. The coherence length of the laser is vastly reduced and the relative intensity noise increases to very high levels.

- Regime V: At high feedback levels (greater than $-10 \mathrm{~dB}$ ), the laser is driven to operate in the extended cavity operation with a narrow linewidth, the linewidth reduction is attributed to the low phase noise associated with this feedback regime. A long cavity is formed by the external reflector with only a short active region. The laser operates on a single longitudinal mode with narrow linewidth for all phases of the feedback. At this regime the feedback dominates the field in the laser, the laser becomes immune to any additional external optical reflections from the fiber transmission line because of the large round trip delay in these compound cavity lasers [10]. The phase of the feedback light suppress the intensity noise of the laser and the intensity noise decreases in this regime.

Figure 2.3 on the next page shows the aforementioned feedback regimes.

The spectra of a DFB laser diode with different feedback levels are shown in Figure 2.4 on page 13. Figure 2.4(a) shows the spectrum of the solitary laser diode operating in a single longitudinal mode. The influence of weak optical feedback is shown in Figure 2.4(b)under which the spectrum shows no change from that of the solitary laser. Multimode operation is reached when the feedback ratio is increased to a level that can drive the laser diode to the coherence collapse regime as shown in Figure 2.4(c). Further increase of the feedback ratio makes the laser again operate in a single mode, Regime V, as shown in Figure 2.4(d). The output power of the laser diode in this regime is higher than that of the solitary laser, this is because the threshold of the laser with feedback is reduced relative to the solitary value. 


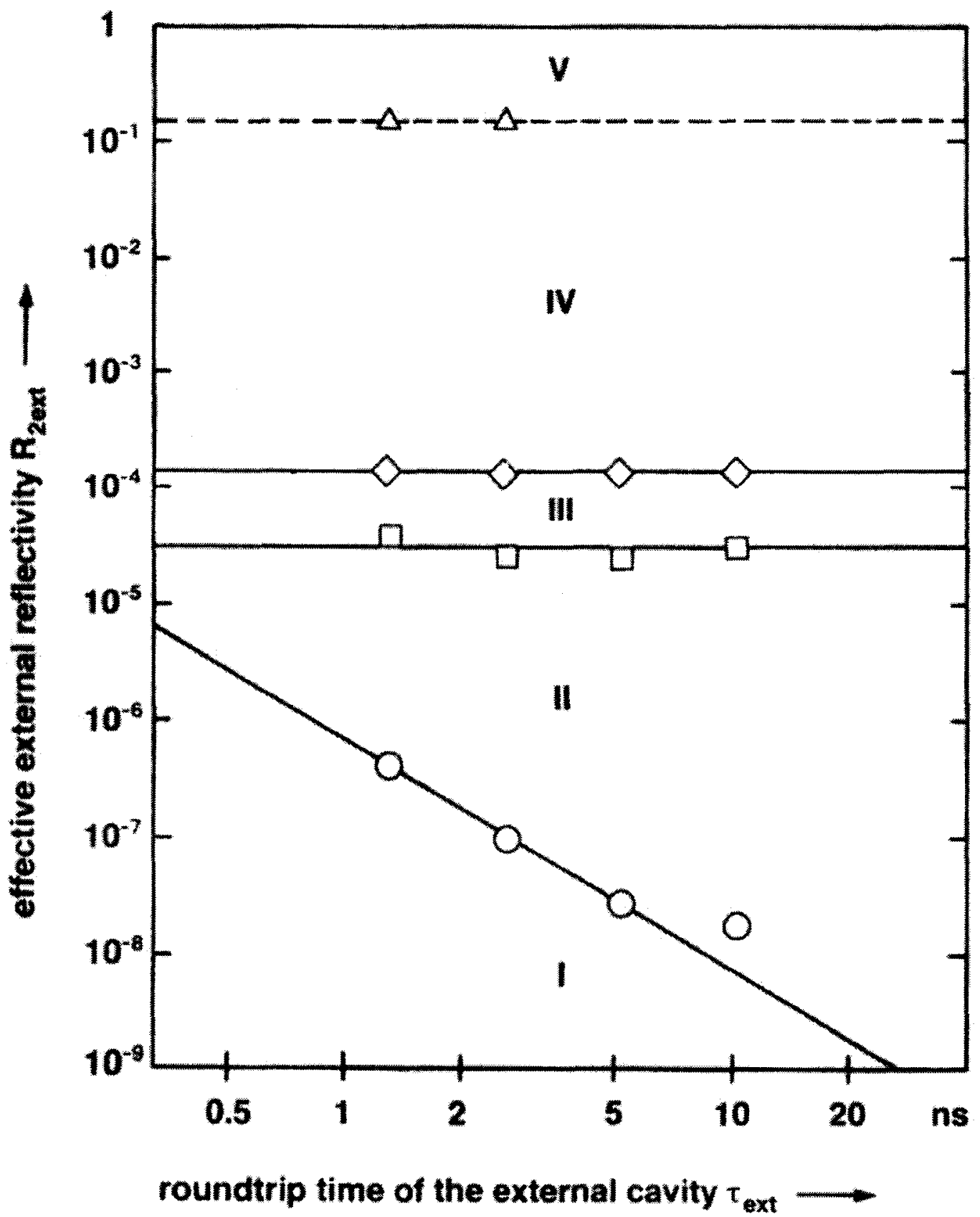

Figure 2.3: Different feedback regimes (Regime I - Regime V) for a DFB laser diode, reproduced from [11]. 

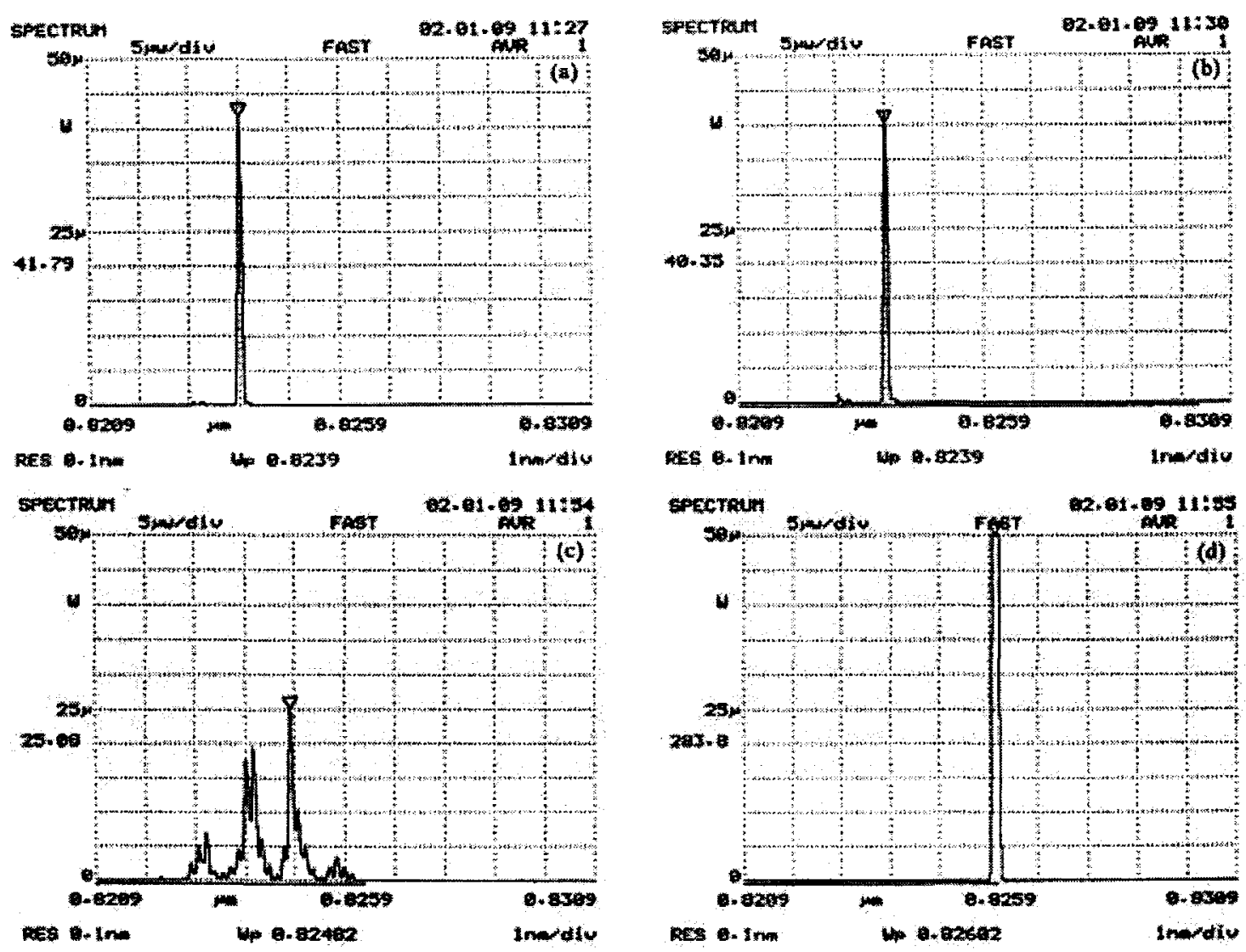

Figure 2.4: DFB laser diode spectra under different feedback levels: (a) the spectrum of the solitary laser diode, (b) DFB laser diode with weak feedback, (c) DFB laser diode operating in coherence collapse regime, and (d) DFB laser diode operating in feedback Regime V, reproduced from [12]. 


\subsection{Conventional Laser Diode Rate Equations Includ- ing Optical Feedback effect}

Optical back-reflections influence semiconductor laser diode nonlinearity and intensity noise, and can modify the static and dynamic characteristics. These reflections originate from the laser facet, laser/fiber interface, and fiber connectors. Rate equations typically neglect optical back-reflections for simplicity, therefore making them ill-suited for analysis of laser diode performance where optical back-reflection is significant. The following is a survey of some of the published work for the conventional laser diode rate equations that include the feedback effect.

Kikushima et al [24] reported an excess harmonic distortion due to the external coherent reflection. They added a term to the laser diode rate equations to describe the backreflected light into the laser, but did not include the time delay of the reflected waves. It was concluded that in order to reduce feedback induced distortion an optical isolator of 30 $\mathrm{dB}$ should be used, this will reduce the reflection coefficient down to $-50 \mathrm{~dB}$.

Czylwik's analysis [25] included the time delay of the reflected waves in the feedback term to predict the upper bound on the harmonic distortion due to reflected waves. $\mathrm{He}$ showed an excess $2^{\text {nd }}, 3^{\text {rd }}$, and $4^{\text {th }}$ order harmonic distortion for the laser diode under -20 $\mathrm{dB}$ and $-30 \mathrm{~dB}$ back-reflection ratios for distances $1 \mathrm{~m}$ and $3 \mathrm{~m}$. However, he did not report on the other feedback regions, nor the laser diode's different behaviors under optical back-reflections.

Helms [26] showed theoretically and experimentally that optical feedback from the fiber enhanced the intermodulation and the harmonic distortion of the laser diode, and this can be achieved if the optical feedback level was kept under $-60 \mathrm{~dB}$.

Cohen et al [27] studied the effect of the optical feedback on the laser diode relaxation oscillation, and found that the relaxation oscillation is modified due to optical feedback.

Agrawal [28] studied the linewidth narrowing in a single-mode injection laser due to external optical feedback, and investigated the effect of fiber-far-end $(7.5 \mathrm{~km})$ reflections on intensity noise and phase noise in InGaAsP semiconductor lasers [29].

Schunk et al [30] measured the feedback-induced intensity noise for a $1.3 \mu \mathrm{m}$ DFB laser diode, the RIN was experimentally measured for a wide feedback range from $-80 \mathrm{~dB}$ 
to $-20 \mathrm{~dB}$. They showed that the transition to the coherence collapse regime occurs at larger feedback levels if the laser is operated at larger optical powers; additionally, a large coupling strength in a DFB laser is beneficial in improving the stability against optical feedback. They also solved the nonlinear rate equations numerically [31] to estimate the external feedback effect on a single-mode semiconductor laser. They reported that the transition to the coherence-collapse regime occurs at a $-40 \mathrm{~dB}$ feedback ratios, and this can be shifted to larger feedback ratio by either increasing the laser output power, laser cavity length, or decreasing the laser linewidth enhancement factor $\alpha$.

Lang and Kobayashi [32] used an external reflector placed a few centimeters apart from the laser diode to investigate the influence of the optical feedback on the semiconductor laser properties. They found that the coherent reflection can either enhance or suppress the relaxation oscillation of the laser depending on interference conditions between returned waves and the field inside the laser diode. They introduced also the so-called Lang/Kobayashi rate equations.

Hirota et al [33] studied the noise properties of the laser diode under direct modulation and influenced by the reflected waves. They estimated the required optical isolation to be $30 \mathrm{~dB}$ to reduce the intensity noise of the laser.

Petermann et al [34] discussed the major sources of noise and distortions in optical fiber communication systems, and concluded that an optical isolator should be included between the laser and the fiber to achieve low distortions and low noise. Petermann attributed the mode hopping and the coherence collapse strong excess noise to the optical feedback phenomena [35].

Favre [36] has shown that the laser facet sensitivity to the optical feedback is related to the emitted power from that facet. It was found that DFB lasers are more sensitive to the feedback than FP lasers. Additionally, DFB lasers with larger $\kappa L$ was found to be relatively insensitive to optical feedback.

Way et al [37] controlled the optical feedback from $-50 \mathrm{~dB}$ to $-25 \mathrm{~dB}$ at the far end of a $2 \mathrm{~m}$ pigtail $1.3 \mu \mathrm{m}$ DFB laser and a multimode laser and compared the linearity performance for both laser diodes. They found that by using suitable bias level and modulation frequency, both of the two types of lasers give good linearity even under strong feedback. 
In [38] Helms et al presented a simple analytic expression describing the maximum feedback level under which the semiconductor laser can operate stably. They also calculated the RIN for several linewidth enhancement factors $\alpha$.

Petermann [39] gave guidelines for designing semiconductor lasers immune to external optical feedback. The mode hopping, linewidth narrowing and broadening, and the transition to the coherence collapse regime due to the weak external optical feedback were reviewed.

Pan et al [40] investigated experimentally the behavior of a semiconductor laser under moderate to strong optical feedback, and showed that the standard Lang/Kobayashi rate equations [32] accurately predict the transition between feedback Regime IV and Regime V.

Jones et al [41] used Lang/Kobayashi rate equations [32] to study the effect of the external cavity length on the coherence collapse regime.

Hong et al [12] showed experimentally the optical spectra of the semiconductor laser subject to different levels of optical feedback. They also calculated the RIN within Regimes I to $\mathrm{V}$. They found that at a low bias current the laser exhibits low RIN with low feedback ratio. The RIN increased too much in the coherence collapse regime (Regime IV) and decreased in Regime $\mathrm{V}$ to a level lower than that of the solitary laser. For higher bias current the transition from Regime IV to $\mathrm{V}$ needs higher feedback ratios.

In 2004, Ju et al [42] numerically calculated the RIN of semiconductor lasers under weak and strong feedback levels to identify the various feedback regimes, the modified Lang/Kobayashi model was used in the calculations.

\subsection{Analysis of Feedback Phenomenon}

The feedback phenomenon in semiconductor lasers can be analyzed based on the model shown in Figure 2.5 on the next page, consisting of laser facet reflection coefficients $r_{1}$ and $r_{2 s}$, the external cavity reflection coefficient $r_{2 e x t}$, and the laser diode cavity length $L$. Introducing an effective reflection coefficient $r_{2}$ at $Z=L$, we obtain [10]:

$$
r_{2}(v)=r_{2 s}+\left(1-\left|r_{2 s}\right|^{2}\right) r_{2 e x t} \exp \left(-j 2 \pi \nu \tau_{\text {ext }}\right)
$$

where $v$ is the optical frequency, and $\tau_{\text {ext }}$ is the round trip delay back and forth through 


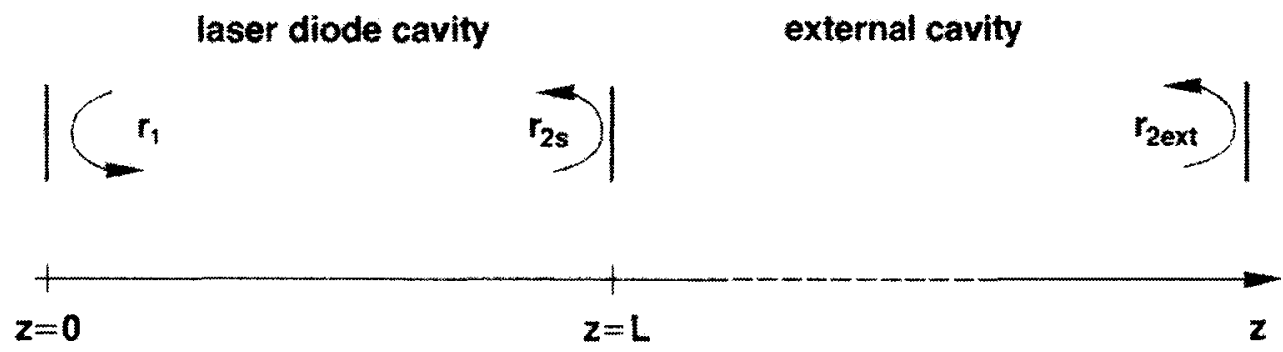

Figure 2.5: Schematic representation of a laser diode cavity with external feedback, reproduced from $[10]$.

the external cavity of length $L_{\text {ext }}$. Also $r_{2}$ can be expressed with respect to amplitude and phase as

$$
r_{2}(v)=\left|r_{2}\right|^{2} \exp \left(-j \phi_{r}\right)
$$

The laser diode phase condition is obtained by equating the round trip phase within the laser diode cavity to an integer multiple of $2 \pi$

$$
2 \beta L+\phi_{r}=2 \pi m
$$

where $m$ is integer and $\beta$ is the propagation constant. For $\phi_{r}=0$, this corresponds to the phase condition for the solitary laser. To obtain the the threshold gain $g=g_{c}$ that satisfies the amplitude condition for the compound cavity laser:

$$
r_{1}\left|r_{2}\right| \exp \left(\left[g_{c}-\alpha_{s}\right] L\right)=1
$$

The feedback strength is classified, based on the ratio between $r_{2 s}$ and $r_{2 e x t}$, into weak feedback $\left|r_{2 e x t}\right| \ll\left|r_{2 s}\right|$ and strong feedback $\left|r_{2 e x t}\right| \gg\left|r_{2 s}\right|$. We will analyze the amplitude and phase condition for the weak feedback case. On the other hand, the analysis of the strong feedback and the dynamics of laser diodes requires a numerical analysis for the laser diode rate equations that include the feedback term. The reflection coefficients $r_{2 s}, r_{2 e x t}$ may be considered as real and positive values, so that (2.6.1) yields 


$$
\left|r_{2}\right|=r_{2 s}\left(1+\kappa_{e x t} \cos \left(2 \pi \nu \tau_{e x t}\right)\right)
$$

where $\kappa_{e x t}$ is the coupling coefficient to the external cavity and is defined as:

$$
\kappa_{e x t}=\frac{r_{2 e x t}}{r_{2 s}}\left(1-\left|r_{2 s}\right|^{2}\right)
$$

and

$$
\phi_{r}=\kappa_{e x t} \sin \left(2 \pi \nu \tau_{e x t}\right)
$$

for $\kappa_{\text {ext }} \ll 1$. There is another feedback coefficient $\mathrm{C}$ that can describe the feedback strength and is defined as:

$$
C=X \sqrt{1+\alpha^{2}}
$$

with

$$
X=\frac{\tau_{e x t}}{\tau_{L}} \kappa_{e x t}
$$

where $\alpha$ is the linewidth enhancement factor, $\tau_{e x t}$ is the round trip delay, back and forth through the external cavity, $\tau_{L}$ is the laser round trip delay. The phase condition in ( 2.6 .3 on the preceding page) may be rewritten in terms of the effective refractive index $\mu_{e}=c \beta /(2 \pi v)$ as

$$
4 \pi \mu_{e} v L / c+\phi_{r}=2 \pi m
$$

The emission frequency $\nu=\nu_{t h}$ is obtained when $\phi_{r}=0$ (without feedback).

The emission frequency, the threshold gain, and the refractive index may change due to weak feedback, yielding a change of $\mu_{e} v$ given by,

$$
\Delta\left(\mu_{e} v\right)=v_{t h} \Delta \mu_{e}+\left(v-v_{t h}\right) \mu_{e}
$$

Expressing (2.6.11) in the phase condition yields

$$
\Delta \phi_{L}=\Delta\left(\mu_{e} v\right) 4 \pi L / c+\phi_{r}=(4 \pi L / c)\left[v_{t h} \Delta \mu_{e}+\mu_{e}\left(v-v_{t h}\right)\right]+\phi_{r}
$$


where $\Delta \phi_{L}$ corresponds to a change in the round trip phase compared to $2 \pi \mathrm{m}$. As the compound cavity round trip phase must equal to $2 \pi \mathrm{m}$, the emission frequencies are obtained from (2.6.12) for $\Delta \phi_{L}=0$ (or $2 \pi$ multiples). The refractive index change $\Delta \mu_{e}$ may be given as

$$
\Delta \mu_{e}=\frac{\partial \mu_{e}}{\partial n}\left(n-n_{t h}\right)+\frac{\partial \mu_{e}}{\partial v}\left(v-v_{t h}\right)
$$

where $n_{t h}$ is the threshold carrier density without feedback. Combining (2.6.12) and (2.6.13) yields

$$
\Delta \phi_{L}=\frac{4 \pi L}{c}\left[v_{t h} \frac{\partial \mu_{e}}{\partial n}\left(n-n_{t h}\right)+\bar{\mu}_{e}\left(v-v_{t h}\right)\right]+\phi_{r}
$$

where $\overline{\mu_{e}}$ is the effective group refractive index.

The linewidth enhancement factor $\alpha$ links the variation of the refractive index with varying carrier density $\frac{\partial \mu_{e}}{\partial n}$ and the gain variations $\frac{\partial g}{\partial n}$ through the following relation [10]:

$$
\frac{\partial \mu_{e}}{\partial n}=\alpha \frac{\partial \mu_{e}^{\prime \prime}}{\partial n}=-\alpha \frac{\partial g}{\partial n} \frac{c}{4 \pi v_{t h}}
$$

with the complex effective refractive index $\mu_{e}=\mu_{e}^{\prime}-j \mu_{e}^{\prime \prime}$, and $\alpha=\delta \mu_{e}^{\prime} / \delta \mu_{e}^{\prime \prime}$. Hence

$$
\frac{\partial \mu_{e}}{\partial n}\left(n-n_{t h}\right)=-\frac{\alpha c}{4 \pi v_{t h}}\left(g-g_{t h}\right)
$$

As the amplitude condition must be satisfied with $g$ equal to the threshold gain for the compound cavity $g_{c},(2.6 .17)$ implies

$$
\Delta \phi_{L}=-\alpha\left(g_{c}-g_{t h}\right) L+\frac{4 \pi \overline{\mu_{e}} L}{c}\left(v-v_{t h}\right)+\phi_{r}
$$

where $g_{t h}$ is the threshold gain without feedback and is given by,

$$
g_{t h}=\alpha_{s}+L^{-1} \ln \left(1 / r_{1} r_{2 s}\right)
$$

The threshold gain difference $\left(g_{c}-g_{t h}\right)$ due to feedback obtained from (2.6.4) and (2.6.5), for $\kappa_{\text {ext }} \ll 1$ is given by,

$$
\left(g_{c}-g_{t h}\right)=-\frac{\kappa_{e x t}}{L} \cos \left(2 \pi \nu \tau_{e x t}\right)
$$


The round trip phase change $\Delta \phi_{L}$ is obtained from (2.6.7), (2.6.17), and (2.6.19) as,

$$
\Delta \phi_{L}=\frac{4 \pi \bar{\mu}_{e} L}{c}\left(v-v_{t h}\right)+\kappa_{e x t}\left[\sin \left(2 \pi v \tau_{e x t}\right)+\alpha \cos \left(2 \pi v \tau_{e x t}\right)\right]
$$

Introducing the round trip delay of the solitary laser cavity $\tau_{L}=2 \overline{\mu_{e}} L / c$, the round trip phase change $\Delta \phi_{L}$ can be rewritten as [10],

$$
\Delta \phi_{L}=2 \pi \tau_{L}\left(\nu-\nu_{t h}\right)+\kappa_{e x t} \sqrt{1+\alpha^{2}} \sin \left(2 \pi \nu \tau_{e x t}+\arctan \alpha\right) .
$$

The laser diode threshold gain is either reduced or increased depending on the phase of the reflected light $\phi_{e x t}=2 \pi \nu \tau_{e x t}$. The maximum reduction occurs when $\phi_{e x t}$ is an integer multiple of $2 \pi$ (in phase feedback).

Figure 2.6 on the next page shows a plot for the round trip phase change $\Delta \phi_{L}$ versus the frequency $\nu-\nu_{t h}$. The phase condition is satisfied when $\Delta \phi_{L}=0$, at which the possible emission frequencies are expected. Without feedback, the round trip phase change $\Delta \phi_{L}$ varies linearly with frequency $\nu$, yielding a single zero at $\nu=\nu_{t h}$. For very small feedback level $C<1$, the round trip phase change $\Delta \phi_{L}$ monotonically increases with $\nu$, again yielding a single zero at $\nu=\nu_{t h}$, i.e., single emission frequency. For high feedback level $C>1$, the round trip phase change $\Delta \phi_{L}$ undergoes strong oscillations yielding multiple zeros for $\Delta \phi_{L}$, at which several external cavity modes around the emission frequency $\nu_{t h}$ of the solitary laser may oscillate.

The above analysis is carried out for FP lasers, it uses the reflectivity $R_{2 s}=r_{2 s}{ }^{2}$ of the laser mirror. To be able to apply the same analysis to DFB lasers, the FP laser mirror reflectivity should be related to the DFB coupling, $\kappa L$, as in Figure 2.2 on page 9.

Equations (2.6.6), (2.6.8), and (2.6.9) govern the feedback sensitivity of laser diodes. For a given external cavity with a given $R_{2 e x t}$ and $\tau_{\text {ext }}$ the feedback sensitivity depends on $R_{2 s}$ and $\tau_{L}$. Larger values for $R_{2 s}$ and $\tau_{L}$ make the laser less sensitive for the feedback due to the low values of $\mathrm{C}$ and $\mathrm{X}$. Given that $\kappa L$ is usually small for DFB lasers, the related effective reflectivity is also small. Additionally, the effective round trip delay for DFB lasers is smaller when compared to that of FP lasers of the same cavity length. DFB lasers are therefore more sensitive to feedback than FP lasers. 


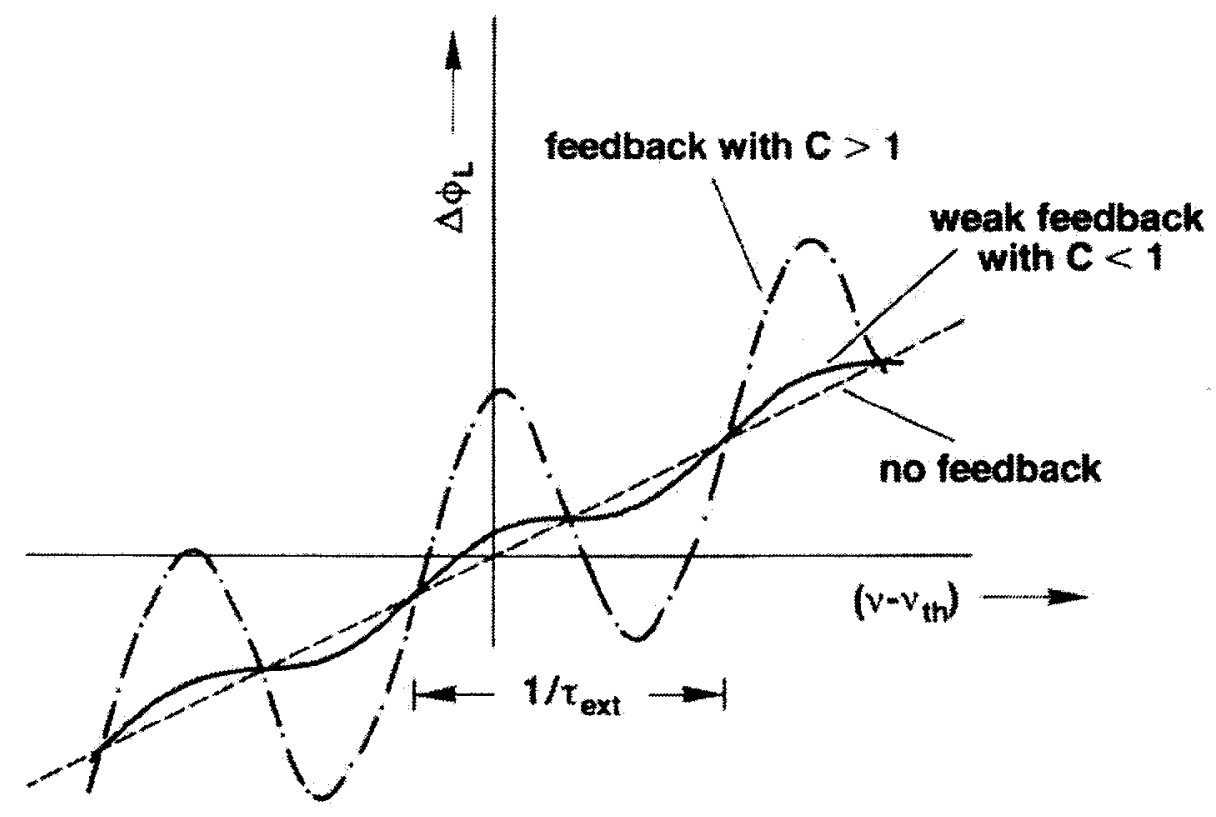

Figure 2.6: Round trip phase change $\Delta \phi_{L}$ versus the optical frequency $\nu-\nu_{\text {th }}$ with no feedback, weak feedback with $C<1$, and feedback with $C>1$, reproduced from [13]. 
DFB laser feedback sensitivity may be reduced for large grating coupling coefficient $\kappa$, but for large $\kappa$, the DFB laser diode external quantum efficiency is reduced. Thus, $\kappa L$ should be chosen carefully.

\subsection{Changes to Laser Performance due to External Cavity}

When coupling the light of the laser diode to an optical fiber, the optical fiber front face represents a reflector, creating an unwanted short external cavity. A tapped fiber end with a hemispherical end face is used to achieve high coupling efficiency and low feedback level. The optimum hemispherical end radius is of the order of $15 \mu \mathrm{m}$, yielding coupling distance of $L_{\text {ext }} \simeq 30 \mu \mathrm{m}$, and external reflectivity of the order of $10^{-5}$ to $10^{-4}$ [43], which is large enough to change the laser diode spectral characteristics.

\subsubsection{Emission Frequency Shift due to Feedback}

The laser diode emission frequency is affected by optical feedback. For short external cavities, the parameter $C$ according to $(2.6 .9$ on page 18$)$ is small $(C<1)$ and $\Delta \phi_{L}$ versus $\nu$ is still a monotonic function. This means that no additional modes due to optical feedback will be of concern. The phase equation ( 2.6 .21 on page 20) is satisfied for $\Delta \phi_{L}=0$ yielding,

$$
\nu-\nu_{t h}=\Delta \nu_{\max } \sin \left(2 \pi \nu \tau_{\text {ext }}+\arctan \alpha\right)
$$

and then the maximum frequency shift due to feedback is given by,

$$
\Delta \nu_{\max }=\frac{\kappa_{e x t} \sqrt{1+\alpha^{2}}}{2 \pi \tau_{L}}
$$

The above equation shows that even with the feedback level as low as $10^{-4}$, facet reflectivity $R_{2 s}=0.32, \kappa_{\text {ext }}=1.2 \times 10^{-2}, \alpha=6$, and $\tau_{L}=10 \mathrm{ps}$, the maximum frequency shift is $\Delta \nu_{\max } \simeq 1.2 \mathrm{GHz}$, which has been observed experimentally in [44]. Even using optical isolators, we cannot guarantee complete removal of the optical back-reflections, and we must still consider the feedback from the isolator's front face. 


\subsubsection{Single External Cavity Mode Condition}

For low external feedback level, the single mode condition (for an arbitrary feedback phase) is satisfied when $C<1$. Larger feedback coefficients may result in a single oscillating frequency if restrictions on $\phi_{e x t}$ are applied. The feedback coefficient $C$ may be tolerated up to $3 \pi / 2$ if the feedback phase is carefully adjusted [10]. For high feedback level, it is still possible for the laser to emit in a single frequency within certain feedback regime.

\subsubsection{Spectral Linewidth Change due to Optical Feedback}

The laser diode spectral linewidth may be influenced considerably due to optical feedback. The spectral linewidth $\Delta \nu$ can be derived from (2.6.21) as [39],

$$
\Delta v \sim\left[d\left(\Delta \phi_{L}\right) / d v\right]^{-2} \quad \text { at } \quad \Delta \phi_{L}=0
$$

yielding,

$$
\Delta v=\frac{\Delta v_{0}}{\left[1+C \cos \left(\phi_{e x t}+\arctan \alpha\right)\right]^{2}}
$$

where $\Delta v_{0}$ is the linewidth of the solitary laser without feedback, and $\phi_{e x t}=2 \pi \nu \tau_{e x t}$. For $C \approx 1$, no additional external cavity modes are introduced. Depending on the feedback phase $\phi_{e x t}$ the linewidth varies between minimum linewidth

$$
\Delta v_{\min }=\frac{\Delta v_{0}}{(1+C)^{2}}
$$

and maximum linewidth

$$
\Delta v_{\max }=\frac{\Delta v_{0}}{(1-C)^{2}}
$$

\subsubsection{Phase and Frequency Noise of Laser with Optical Back- reflections}

For low feedback levels, the frequency noise spectrum can be evaluated analytically as described in [45]. For high levels of feedback, a numerical analysis for the laser diode rate equations is required to evaluate the frequency noise. Figure 2.7 on the next page 
shows the calculated frequency noise spectrum for a $1 \mathrm{~m}$ long external cavity at a feedback ratio of $2.5 \times 10^{-5}$, this amount of feedback corresponds to Regime III. The frequency noise is suppressed and its spectrum shows a white noise behavior for frequencies lower than the inverse external round trip time $1 / \tau_{\text {ext }}$, yielding the strong linewidth narrowing. The frequency noise spectrum exhibits peaks at frequencies corresponding to multiples of $1 / \tau_{\text {ext }}$.

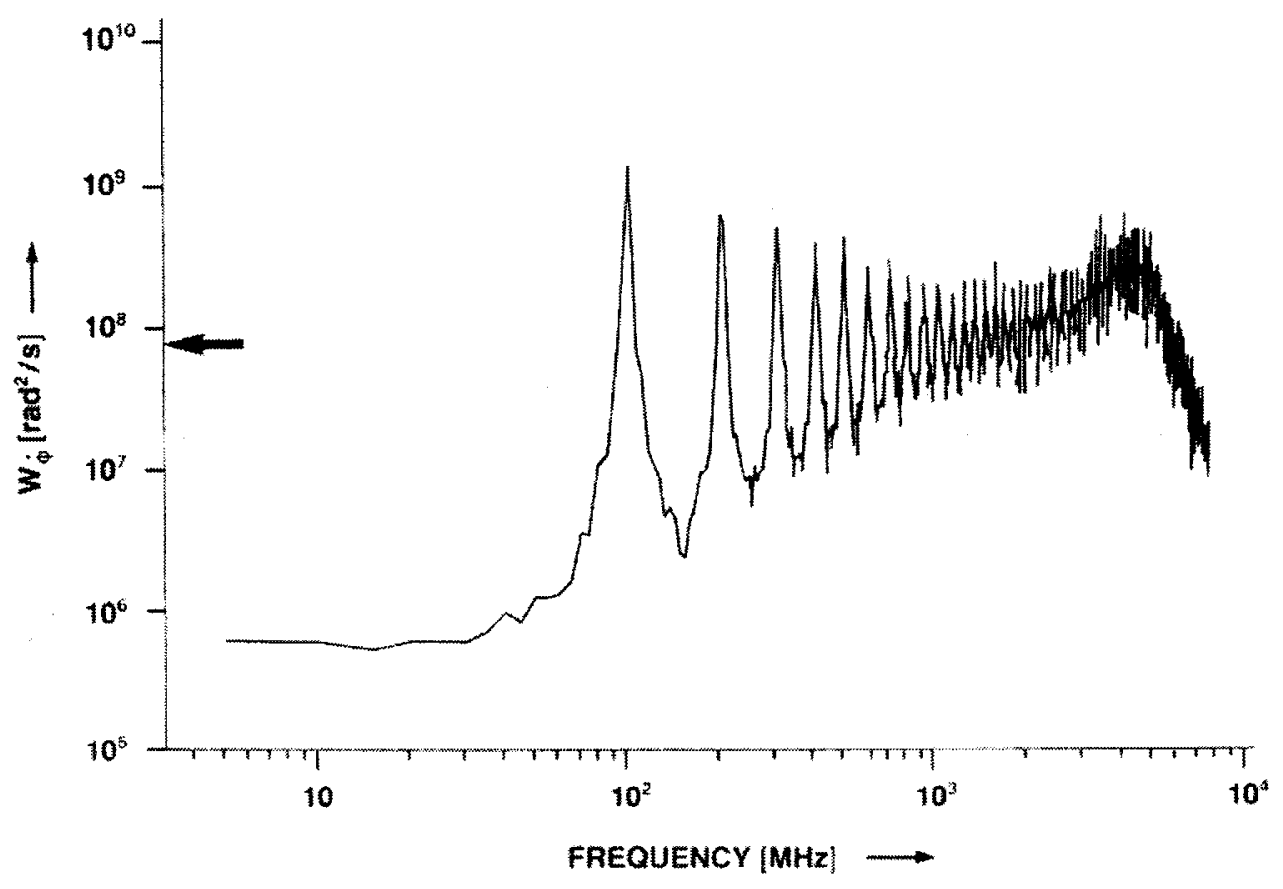

Figure 2.7: Calculated frequency noise spectrum for $1 \mathrm{~m}$ long external cavity at a feedback fraction of $2.5 \times 10^{-5}$ for the solitary laser diode, reproduced from [46].

\subsubsection{Laser Intensity Noise due to Optical Feedback}

Considering a single mode DFB laser diode with optical feedback, a numerical analysis for the laser diode rate equations yields the RIN shown in Figure 2.8 on page 26. RIN remains relatively low for $R_{2 e x t}<10^{-4}$, and it drastically increases for $R_{2 e x t}>10^{-4}$. The sharp increase in the RIN is due to the transition between Regimes III and IV. Any further increase in the feedback level drives the laser to feedback Regime V, at which the RIN is decreased to a value lower than that of the solitary laser, as shown in Figure 2.9 for the 
measured RIN at different feedback levels [12].

\subsubsection{Dynamic Properties of Laser with External Cavity}

The modulation characteristics of external cavity lasers can be described by the laser diode rate equations that include the optical back-reflections term. The modulation characteristics of the external cavity laser with very short external cavity lengths (a few hundred microns) are similar to those of solitary lasers, almost no change due to the optical backreflections. For external cavity length ranges from few millimeters up to $2 \mathrm{~cm}$, and depending on the reflected light phase, an enhancement or damping of the relaxation oscillations may occur due to the optical back-reflections.

\subsection{CATV Systems}

Cable Television (CATV) system uses bi-directional signal transmission in order to enable the distribution of TV signals and a range of various other services such as radio and satellite signals. The bi-directional communication nature of CATV makes it possible to receive signals (TV, radio program, etc) passively as well as enable users to select the type and content of the offered services.

Today's CATV networks use a Hybrid Fiber/Coax (HFC) configuration to reduce cost while increasing performance and transmission range. This is achieved by exploiting the large bandwidth and low loss offered by optical fibers. An HFC network uses coaxial cable for shorter transmission lengths often between video equipment and the transmitter, or the receiver. On the other hand, the transmitter and the receiver are connected using singlemode optical fiber in order to extend the transmission distances by orders of magnitude, thereby reducing the number of RF amplifiers (spaced every 300-600 m) often associated with coaxial cables. This combination of coaxial cables and optical fibers allows system designers to employ the lowest cost solution for each portion of the network. CATV systems can carry more than 100 analog channels spaced at $6 \mathrm{MHz}$ intervals.

A typical CATV system comprises a CATV transmitter and receiver. Figure 2.10 on page 28 illustrates the basic structure of a HFC CATV system. A CATV receiver contains a PIN detector which is considered one of the most linear optical components. A CATV 


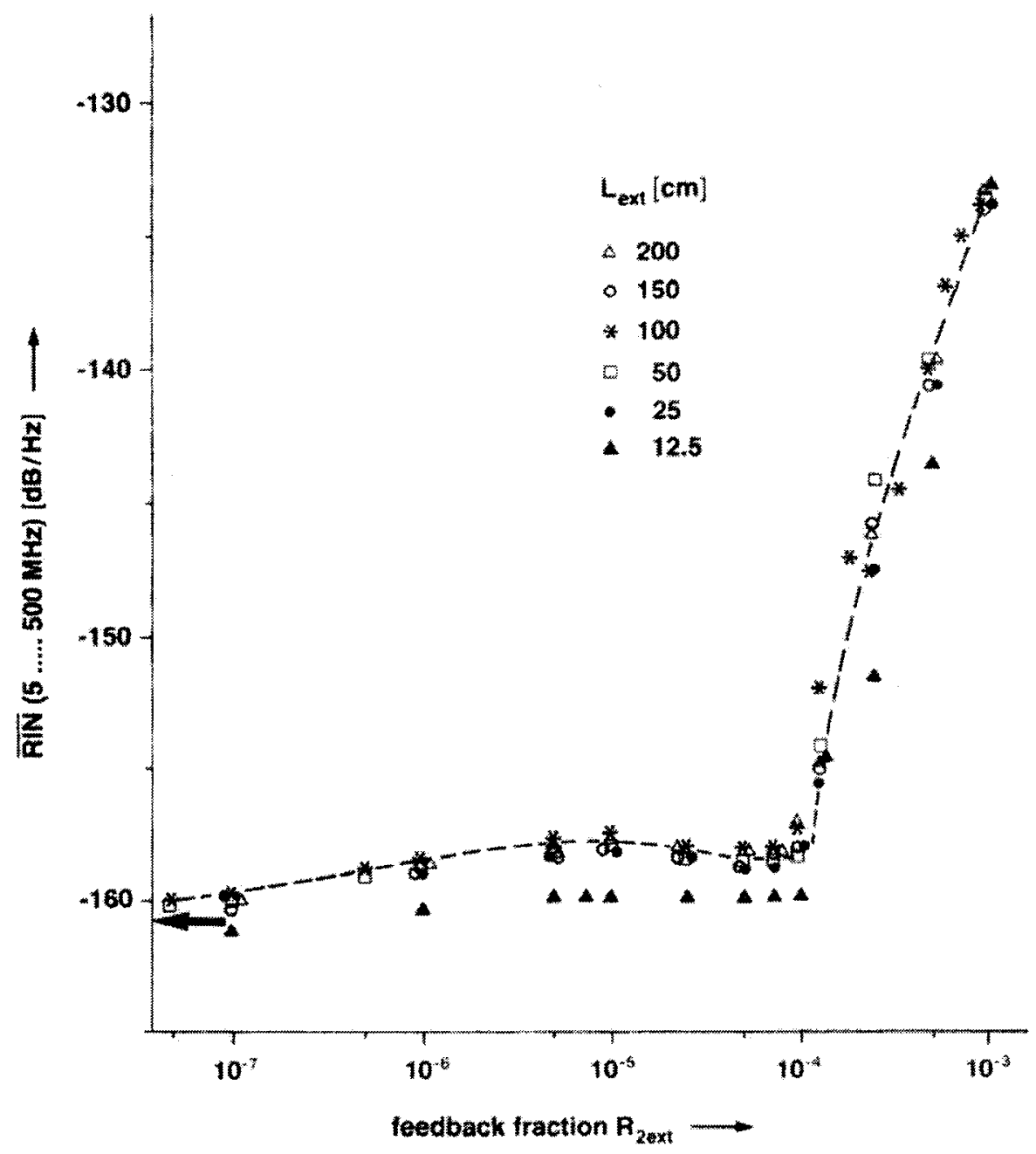

Figure 2.8: Calculated relative intensity noise averaged over the frequency range $5 \cdots 500$ $\mathrm{MHz}$ versus the feedback fraction for various external cavity lengths at an output power $(5 \mathrm{~mW})$, reproduced from [31]. 


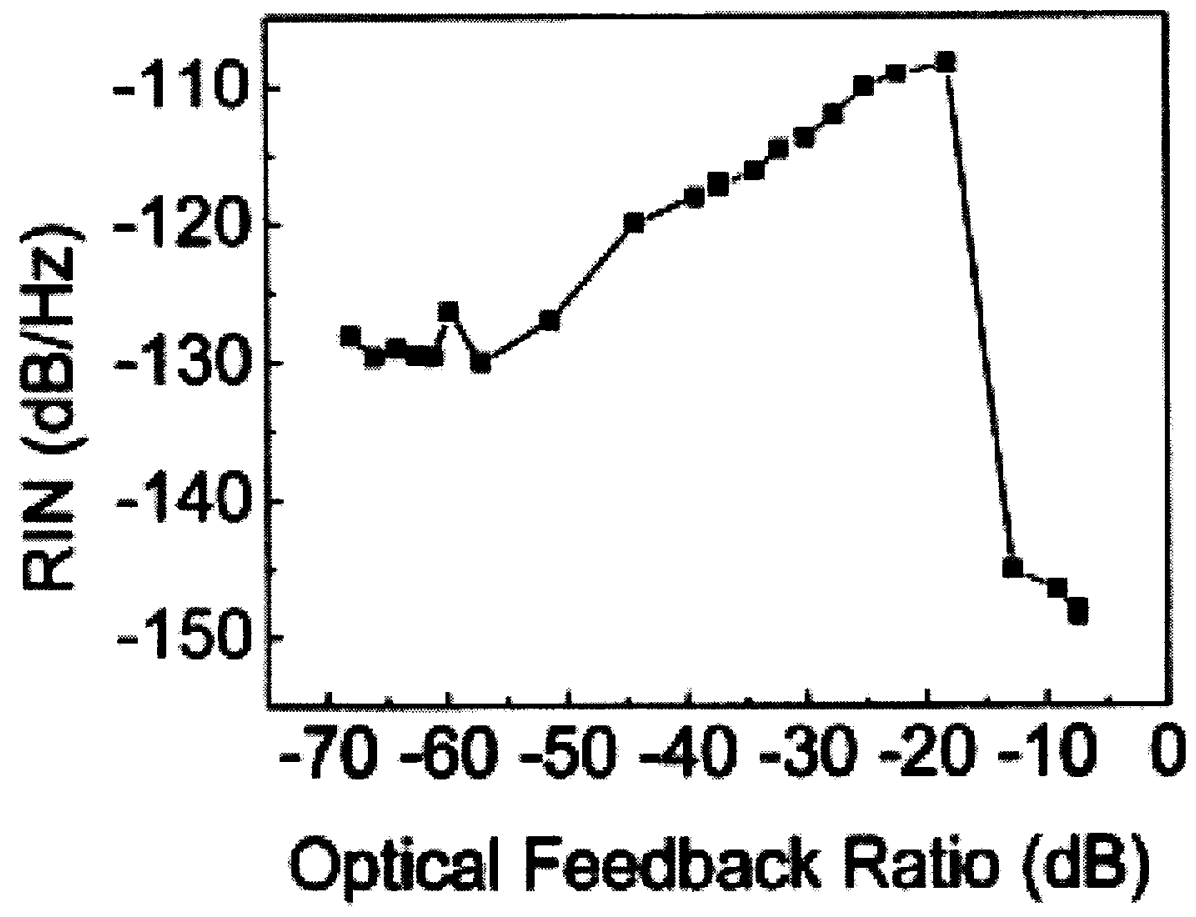

Figure 2.9: Measured relative intensity noise as a function of feedback ratio with $29.3 \mathrm{~mA}$ laser bias current, reproduced from [12]. 
transmitter employs a laser diode; the biggest challenge usually lies within the nonlinear nature of the laser. $1310 \mathrm{~nm}$ DFB lasers dominated CATV transmission for many years. Many enhancements to improve the lasers' linearity as well as to lower their Relative Intensity Noise (RIN) were implemented. Recently, the popularity of $1550 \mathrm{~nm}$ wavelength allows transmission distance to increase from $20-30 \mathrm{~km}$ to $60-70 \mathrm{~km}$ [1]. Nonetheless, nonlinear distortions and intensity noise introduced by lasers can degrade the system performance as well as limit the maximum transmission distance.

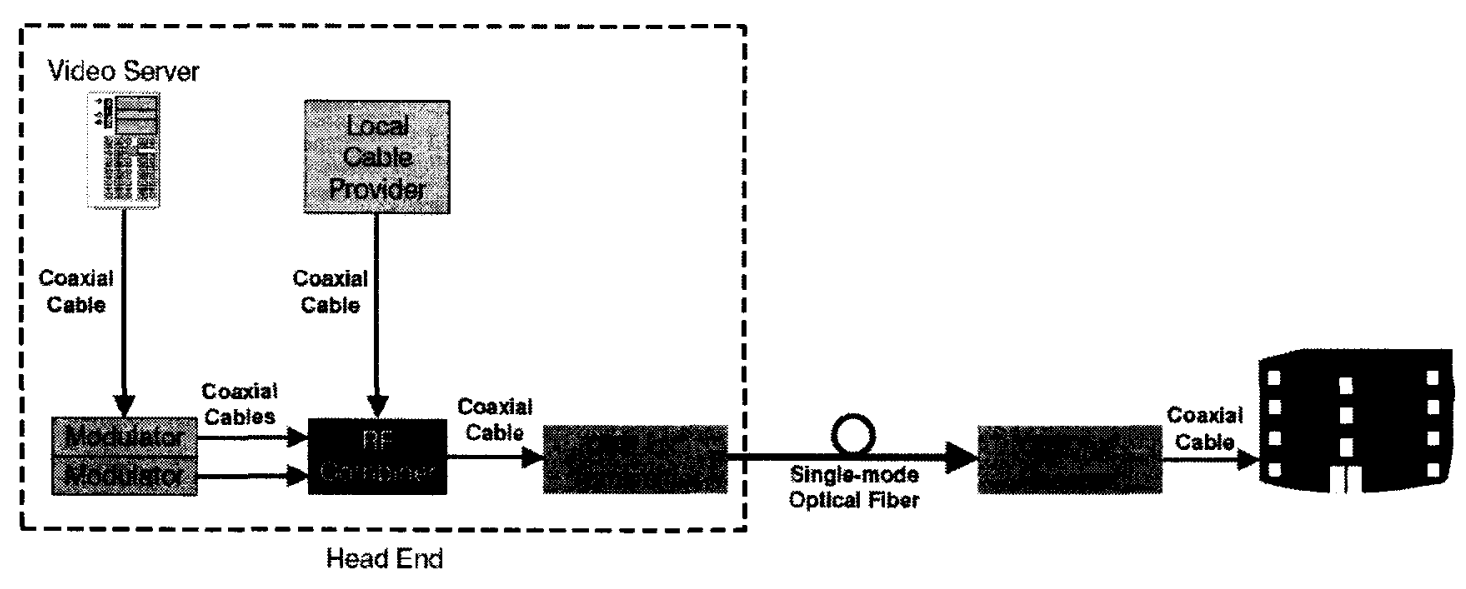

Figure 2.10: Basic structure of a Hybrid Fiber/Coax 1310/1550 nm CATV system. Reproduced from Force Incorporated (www.forceinc.com).

\subsubsection{CATV Transmitter Performance}

In this section we will discuss the performance of CATV transmitter. We will start with the laser noise and then discuss how the laser linearity affect the CATV performance. A typical CATV transmitter specifications is given at the end of the section.

\section{CATV Transmitter Noise}

Carrier-to-Noise Ratio (CNR) is defined as the ratio of the carrier to the total noise power in a $4 \mathrm{MHz}$ bandwidth. Analog CATV signals require a CNR around $50 \mathrm{~dB}$ for acceptable picture quality. RIN from the laser is one of the major factors that limit the CNR. Low RIN is highly recommended for the DFB lasers in order to achieve the desired CRN for 
analog CATV systems [4].

\section{CATV Transmitter Linearity}

Composite Triple Beat (CTB) and Composite Second Order (CSO) are two important measures of linearity in wideband systems handling many signals simultaneously such as CATV systems. To characterize CATV system linearity, N signals of equal power level are applied to the system spaced $6 \mathrm{MHz}$ apart [47].

Considering three signals of equal amplitude, $x_{1}, x_{2}$, and $x_{3}$, the third order nonlinearity results in:

$$
\begin{aligned}
\left(x_{1}+x_{2}+x_{3}\right)^{3} & =x_{1}^{3}+x_{2}^{3}+x_{3}^{3} \\
+ & 3 x_{1}^{2} x_{2}+3 x_{1}^{2} x_{3}+3 x_{2}^{2} x_{1}+3 x_{3}^{2} x_{1}+3 x_{2}^{2} x_{3}+3 x_{3}^{2} x_{2} \\
& +6 x_{1} x_{2} x_{3}
\end{aligned}
$$

The first line of (2.8.1) causes third order harmonics (HD3), second line causes third order intermodulation (IM3), and last line causes CTB in that it creates terms at frequencies $\left(f_{1} \pm f_{2} \pm f_{3}\right)$. Except for the case where all three frequencies are added $\left(f_{1}+f_{2}+f_{3}\right)$, these tones can fill into any of the channels being used and many will fall into the same channel.

Similar to CTB, CSO is used to measure CATV system linearity. For N signals of same power, consider the second order distortion products of each pair of signals falling at frequencies $\left(f_{1} \pm f_{2}\right)$. If the carriers are not some even multiple of the channel spacing, the signals fall at frequencies either above or below the carriers instead of falling right on top of them as in the case of the triple-beat terms.

The Intercept Point is a common distortion specification for nonlinear systems. If the output versus input of a device is displayed graphically on a logarithmic versus logarithmic scale, the slope of the linear portion will be "1". If second and third order distortion products are displayed on the same scale, they will have a slope of "2" and " 3 " respectively. The third order intercept point, $I P 3$, is a theoretical point where the amplitudes of the intermodulation tones at $2 f_{1}-f_{2}$ and $2 f_{2}-f_{1}$ are equal to the amplitudes of the fundamental tones at $f_{1}$ and $f_{2}$. The power at this point, $P_{I P 3}$, is given by, 


$$
P_{I P 3}=P_{i}+\frac{1}{2}\left[P_{1}-P_{3}\right]
$$

where, $P_{i}$ is the input signal power, $P_{1}$ is the output power at the fundamental frequency, and $P_{3}$ is the output power at the IM3 frequency.

Similar to $I P 3$, the second order intercept point, IP2, can be defined in a similar way. The power at this point, $P_{I P 2}$, is given by,

$$
P_{I P 2}=P_{i}+\frac{1}{2}\left[P_{1}-P_{2}\right]
$$

where $P_{2}$ is the output power at the second order intermodulation frequency, IM2.

The power of the Triple Beat (TB) and the Second Order beat (SO) tones are given by (2.8.4) and (2.8.5) respectively [47].

$$
\begin{gathered}
T B(d B m)=P_{I P 3}-3\left(P_{I P 3}-P_{s}\right)+6 \\
S O(d B m)=P_{I P 2}-2\left(P_{I P 2}-P_{s}\right)
\end{gathered}
$$

where $P_{s}$ is the fundamental tone signal power. It is highly desirable to decrease the level of the TB and SO tones in order to get CTB and CSO distortions to a minimum. This can be achieved by deceasing second and third order intermodulation distortions, $P_{2}$ and $P_{3}$, consequently increasing $P_{I P 2}$ and $P_{I P 3}$. This will decrease the TB and SO levels. To meet CSO and CTB requirements for CATV systems, the laser's second order harmonic distortion and third order intermodulation distortion of types $\left(2 f_{1} \pm f_{2}\right.$ and $\left.2 f_{2} \pm f_{1}\right)$ must be in the vicinity of $-75 \mathrm{dBc}$ and $-100 \mathrm{dBc}$ respectively, for a modulation index of 0.04 [4].

\section{Typical CATV Transmitter Performance}

Table 2.1 on the following page lists the typical CATV transmitter related specifications. Carrier-to-Noise Ratio (CNR) is determined both by the number of transmitted channels, as well as the received optical power at the input of the receiver. CNR is highly affected by the laser RIN; increasing the RIN decreases the CNR. Composite Second Order (CSO) and Composite Triple Beat (CTB) are measures of CATV transmitter linearity. Both attributes are affected by various types of laser distortions; increasing a laser's second order harmonic 
distortion, third order harmonic distortion, or intermodulation distortions, increases CSO and CTB.

\begin{tabular}{|c|c|c|c|c|}
\hline Specification & Minimum & Typical & Maximum & Units \\
\hline \hline Channel Loading & & & 110 & Channels \\
\hline Operating Wavelength & 1290 & 1310 & 1330 & $\mathrm{~nm}$ \\
\hline Optical Output Power & +6 & & +13 & $\mathrm{dBm}$ \\
\hline Required Fiber Bandwidth & 1,000 & & & $\mathrm{MHz}$ \\
\hline CNR & & 50 & & $\mathrm{~dB}$ \\
\hline CSO & & -64 & -62 & $\mathrm{~dB}$ \\
\hline CTB & & -69 & -67 & $\mathrm{~dB}$ \\
\hline Side Mode Suppression Ratio & & 30 & & $\mathrm{~dB}$ \\
\hline Back-reflection Tolerance & & & -50 & $\mathrm{~dB}$ \\
\hline Operating Temperature Range & 0 & & 45 & ${ }^{\circ} \mathrm{C}$ \\
\hline
\end{tabular}

Table 2.1: Typical CATV transmitter specifications, reproduced after [1].

\subsubsection{Limiting Factors to CATV System Performance}

Many factors limit the performance of CATV systems. Most important of these factors include:

- Laser nonlinearity.

- Optical back-reflections in the fiber plant.

- Fiber nonlinearities.

- Stimulated Brillouin scattering.

- Fiber-induced relative intensity noise.

In this section we will discuss the first two factors. Over the years, laser manufacturers have developed chip designs that have improved linearity [1]. Today one can find $10 \mathrm{dBm}$ lasers that can carry 77 or more analog channels and deliver CSO and CTB better than $-55 \mathrm{~dB}$. However, there is a price to pay for this performance; high linearity lasers require extensive screening that results in low yields.

Several linearization techniques were reviewed in [19] to compensate for distortions these included: optical feedback and optical feed-forward, post-distortion, and pre-distortion. The analysis made in [19] supported the use of pre-distortion techniques. The laser linearity 
can be improved using predistortion circuits. Complex predistortion circuits have a wide room for adjustments. Hence, each laser has its own custom-tuned predistortion board.

The CNR of a CATV system is limited by the laser's RIN. One major factor increasing the RIN and distortion is optical back-reflections which can modify the static and dynamic characteristics of the laser. These reflections originate from the laser facet, laser/fiber interface, and fiber connectors. Optical isolators were used to reduced the level of the reflected optical power. The use of optical isolators cannot completely remove the optical feedback effect since the built-in isolators typically allow for an isolation level of around $30 \mathrm{~dB}$, which is insufficient in some applications.

\subsection{Motivation}

Most current DFB laser modules contain a built-in optical isolator to reduce the noise and distortion induced by optical back-reflections. The optical isolator increases costs and complicates the construction of multi-function integrated devices. Extra time is required to optically align the isolator. An uncooled, isolator free laser module is desirable for low cost lasers. A significant amount of previous research aimed at removing the isolator [48-52].

Hence, a need for a comprehensive laser model which has the ability to predict the laser diode performance due to the reflected waves is required.

The laser spectrum is narrowed for all feedback phases only in Regime V; it becomes insensitive to further reflections. In general this regime cannot be achieved for uncoated lasers without intentional external reflections. Otherwise the laser will suffer a degradation due to unintended reflections from splices and connectors (typically -30 to $-40 \mathrm{~dB}$ ). The 30 $\mathrm{dB}$ isolators will not be enough to prevent the broadening of the spectral linewidth [11].

The challenge is to force the laser to operate in Regime V. FBG provides a good solution: including a suitable external FBG in the fiber pigtail can drive the laser diode to operate in Regime V. The FBG can be implemented using a simple and cheap technique. The laser output power reduction due to the presence of the grating is self-compensated as the optical feedback from the grating reduces the laser threshold as will be shown in Section 4.2.1.

To meet linearity requirements for CATV systems, an effective linearization technique 
is required to compensate for the laser's nonlinearity. This linearization technique should take into account major causes for the laser nonlinearity.

In this work, two major objectives were accomplished. Firstly, improve laser RIN and hence achieve the desired CNR required for CATV systems. Secondly, achieve lower levels of CTB and CSO by decreasing the various laser harmonic and intermodulation distortions. The first objective was achieved by a feedback topology that favorably applies optical feedback to the DFB laser in order to overcome the feedback induced RIN and get rid of the optical isolator. Since existing laser models do not enable designers to properly simulate laser with optical back-reflection, laser rate equations that include the optical feedback effect were implemented. This enabled us to build a laser model that has the ability to analyze various laser performance parameters taking into account optical feedback. Furthermore, based on the proposed rate equations, we developed a laser predistorter model. Simulation results shows that a considerable improvement in the laser's linearity was achieved when the laser model was cascaded to the predistorter. This improvement makes the laser suitable for CATV applications, hence fulfilling our second set objective. 


\section{Chapter 3}

\section{Proposed Model and Simulation Results}

\subsection{Introduction}

Introducing the effects of optical back-reflections to the laser diode rate equations came with the challenge of solving the resulting complicated rate equations. Attempts to find numerical solutions met convergence difficulty. Such convergence problems can be avoided with a suitable normalization technique that can enhance the convergence of numerical solvers. In this chapter the proposed rate equations which include the optical backreflection effects are introduced. The introduced feedback terms are derived and normalized. An Agilent-ADS model for system level simulation will be implemented based on the new proposed rate equations. $\mathrm{DC}$, transient, and harmonic balance simulation results will be demonstrated.

\subsection{The Proposed Rate Equations}

The analysis of a laser diode with optical back-reflections involves comprehensive rate equations that have the ability to describe linearity and noise characteristics. Ghoniemy et al [19] introduced a comprehensive laser model that included semiconductor laser gain modifications and the effects of device heating, current leakage, and noise. This model has been augmented to include feedback terms that account for optical back-reflections phenomenon. Two different feedback terms have been added to the photon rate equation and phase rate equation; the electron rate equation remains unchanged. 


\subsubsection{Derivation of the Feedback Terms}

Consider the model shown in Figure 2.5 on page 17. Starting from the first principles and using the technique presented in [10], the laser oscillation occurs when:

$$
r_{1} r_{2} \exp \left(-2 j \beta L+\left(g-\alpha_{s}\right) L\right)=1
$$

with:

$$
r_{2}=\left|r_{2}\right| \exp \left(-j \phi_{r}\right)=r_{2 s}+\left(1-\left|r_{2 s}\right|^{2}\right) r_{2 e x t} \exp \left(-j 2 \pi \nu \tau_{\text {ext }}\right)
$$

where $r_{1}$ and $r_{2 s}$ are the laser facet reflection coefficients, $r_{2}$ is the effective reflection coefficient at $Z=L, \mathrm{~g}$ in this equation accounts for the compound cavity stimulated emission gain, $\alpha_{s}$ is the optical loss (scattering loss), $\mathrm{L}$ is the laser cavity length, $\phi_{r}$ is the reflected power phase, and $\beta$ is the optical wave phase constant. The $\left(1-\left|r_{2 s}\right|^{2}\right)$ term represents the light transmission through the exit facet of the laser diode.

The required gain condition is obtained from the absolute value of (3.2.1), while the phase condition is obtained from the phase of (3.2.1). With real values for $r_{1}=\sqrt{R_{1}}$ and $r_{2}=\sqrt{R_{2}}$, where $R_{1}$ and $R_{2}$ are the laser facets' power reflectivities, the phase and amplitude conditions of the compound cavity are given by (2.6.3 on page 17) and ( 2.6 .4 on page 17) respectively.

Considering the case of a solitary laser diode for the moment, the phase constant, $\beta$, depends on the optical frequency $\nu$ and the possible emission frequencies are obtained from (2.6.3) (with $\phi_{r}=0$ ). Introducing the effective refractive index $\mu_{e}$ for the lasing mode as:

$$
\mu_{e}=c \frac{\beta}{2 \pi \nu}
$$

and the possible frequencies are:

$$
\nu=\frac{m c}{2 L \mu_{e}}
$$

The effective group refractive index $\bar{\mu}_{e}$ is given by

$$
\overline{\mu_{e}}=\mu_{e}+\frac{d \mu_{e}}{d \nu}
$$


The spacing between two adjacent emission frequencies $\delta \nu$ is defined by

$$
\delta \nu=\frac{c}{2 L \overline{\mu_{e}}}
$$

The round trip time delay $\tau_{L}$ corresponds to the inverse of the spacing between adjacent emission frequencies $1 / \delta \nu$, therfore,

$$
\tau_{L}=\frac{2 L \overline{\mu_{e}}}{c}
$$

Introducing the carrier density at the lasing threshold, $n_{t h}, g\left(n_{t h}\right)=g_{t h}$, and because of the dependance of the effective refractive index, $\mu_{e}$, on the optical frequency and the carrier density, the resonance frequency, $\nu_{t h}$, at $n=n_{t h}$ is obtained from:

$$
\nu_{t h}=\frac{m c}{2 L \mu_{e}\left(\nu_{t h}, n_{t h}\right)} .
$$

If the carrier density deviates slightly from $n_{t h}$, the effective refractive index $\mu_{e}$ can be expanded in terms of $\nu_{t h}$ and $n_{t h}$, yielding:

$$
\mu_{e}=\mu_{e}\left(\nu_{t h}, n_{t h}\right)+\frac{\partial \mu_{e}}{\partial \nu}\left(\nu-\nu_{t h}\right)+\frac{\partial \mu_{e}}{\partial n}\left(n-n_{t h}\right) .
$$

Combining (3.2.4), (3.2.8), and (3.2.9) gives:

$$
\left(\nu-\nu_{t h}\right)=\frac{\nu_{t h}}{\bar{\mu}_{e}} \frac{\partial \mu_{e}}{\partial n}\left(n-n_{t h}\right)
$$

The round trip gain, $\mathrm{G}$, is given by:

$$
G=r_{1} r_{2} \exp \left(-2 j \beta L+\left(g-\alpha_{s}\right) L\right) .
$$

Expanding $\beta=\frac{\omega}{c} \mu_{c}$ in terms of $\omega_{t h}$ and $n_{t h}$, and in a fashion similar to (3.2.9) yields:

$$
\beta=\frac{\omega_{t h}}{c}\left(\mu_{e}\left(\nu_{t h}, n_{t h}\right)+\frac{\partial \mu_{e}}{\partial n}\left(n-n_{t h}\right)+\frac{\overline{\mu_{e}}}{\omega_{t h}}\left(\omega-\omega_{t h}\right)\right)
$$

The parameters: $\mathrm{g}, \alpha_{s}, r_{1}$, and $r_{2}$ are frequency independent parameters. Substituting with (3.2.12) in (3.2.11), the round trip gain, G, can be written in terms of two different 
terms: Firstly, frequency independent term $G_{1}$ :

$$
G_{1}=\sqrt{R_{1} R_{2}} \exp ([g-\alpha s] L) \exp \left(-j\left[\left(2 \omega_{t h} L / c\right) \frac{\partial \mu_{e}}{\partial n}\left(n-n_{t h}\right)\right]\right)
$$

and secondly frequency dependent term $G_{2}$ :

$$
G_{2}=\exp \left(-\frac{2 j \omega_{t h} L}{c}\left[\mu_{e}\left(\nu_{t h}, n_{t h}\right)+\frac{\bar{\mu}_{e}}{\omega_{t h}}\left(\omega-\omega_{t h}\right)\right]\right)
$$

The electric field within the laser cavity is characterized by the complex amplitude $E(t)$ :

$$
E(t)=\sqrt{P(t)} \exp (j \phi(t))
$$

and the actual electrical field is given by $\operatorname{Re}\left(E(t) \exp \left(j \omega_{t h} t\right)\right)$, where $P(t)$ is the photon number, and $\phi(t)$ is the phase of the slowly varying amplitude of the forward traveling wave in the laser cavity. Neglecting the spontaneous emission term, the time evolution of electric field, $E(t)$, can be written as:

$$
E(t)=G_{1} E\left(t-\tau_{L}\right)
$$

As $E(t)$ is a slowly varying field amplitude, then the variations during the round trip delay, $\tau_{L}$, will be small, yielding:

$$
E\left(t-\tau_{L}\right)=E(t)-\tau_{L} \frac{d E(t)}{d t}
$$

and then

$$
\frac{d E(t)}{d t}=\frac{1}{\tau_{L}}\left(1-1 / G_{1}\right) E(t)
$$

To achieve the lasing condition the round trip gain should be close to unity, so that the exponential expression in (3.2.13) gives:

$$
\frac{1}{G_{1}} \approx 1+\frac{1}{2} \ln \left(1 / R_{1} R_{2}\right)-g L+\alpha_{s} L+j\left[\left(2 \omega_{t h} L / c\right) \frac{\partial \mu_{e}}{\partial n}\left(n-n_{t h}\right)\right] .
$$

Substituting with (3.2.19) in (3.2.18) gives:

$$
\frac{d E(t)}{d t}=\left(j \frac{\left(2 \omega_{t h} L / c\right) \frac{\partial \mu_{e}}{\partial n}\left(n-n_{t h}\right)}{\tau_{L}}+\frac{g L}{\tau_{L}}-\frac{\alpha_{s} L+(1 / 2) \ln \left(1 / R_{1} R_{2}\right)}{\tau_{L}}\right) E(t)
$$


The group velocity of an optical wave is given by $v_{g}=c / \overline{\mu_{e}}=2 L / \tau_{L}$, and using (3.2.10) yields $\left(2 \omega_{t h} L / c\right) \frac{\partial \mu_{e}}{\partial n}\left(n-n_{t h}\right) / \tau_{L}=2 \pi\left(\nu-\nu_{t h}\right)=\omega-\omega_{t h}$, with $\omega$ representing the actual resonance frequency of the laser cavity. Including the feedback term yields:

$$
\frac{d E(t)}{d t}=\left(j\left(\omega-\omega_{t h}\right)+\frac{1}{2}\left[g\left(\frac{c}{\bar{\mu}_{e}}\right)-\frac{1}{\tau_{p h}}\right]\right) E(t)+\frac{1}{\tau_{L}} \kappa_{e x t} E\left(t-\tau_{e x t}\right) \exp \left(-j \omega_{t h} \tau_{e x t}\right)
$$

where $\tau_{p h}$ is the photon lifetime of the solitary laser and is defined by:

$$
\frac{1}{\tau_{p h}}=\left(c / \overline{\mu_{e}}\right)\left(\alpha_{s}+\frac{1}{2 L} \ln \left(\frac{1}{R_{1} R_{2}}\right)\right)
$$

Using (3.2.7) the photon lifetime $\tau_{p h}$ can be written as:

$$
\tau_{p h}=\frac{\tau_{L}}{2 L g_{t h}} .
$$

For $\frac{d P}{d t}=2 \operatorname{Re}\left(E^{*}(t) \frac{d E}{d t}\right)$ and $\frac{d \phi}{d t}=\operatorname{Im}\left(E^{*}(t) \frac{d E}{d t}\right) / P(t),(3.2 .15)$ yields:

$$
\frac{2}{\tau_{L}} \kappa_{e x t} \sqrt{P(t) P\left(t-\tau_{e x t}\right)} \cos \left(\omega_{t h} \tau_{e x t}+\phi(t)-\phi\left(t-\tau_{e x t}\right)\right)
$$

for the photon rate equation feedback term and

$$
-\frac{1}{\tau_{L}} \kappa_{e x t} \frac{\sqrt{P\left(t-\tau_{e x t}\right)}}{\sqrt{P(t)}} \cdot \sin \left(\omega_{t h} \tau_{e x t}+\phi(t)-\phi\left(t-\tau_{e x t}\right)\right)
$$

for the phase rate equation feedback term.

\subsubsection{Feedback Term Normalization and the Proposed Rate Equa- tions}

The normalization techniques enhance the convergence of the numerical solvers and make them suitable for transient, small signal, and large signal analysis. Applying the normalization methodology introduced by Ghoniemy et al [19] on the unnormalized photon rate equation feedback term given by (3.2.24) yields:

$$
\frac{2}{\tau_{L}} \kappa_{e x t} \sqrt{P(t) P\left(t-\tau_{e x t}\right)} \cos \left(\omega_{t h} \tau_{e x t}+\phi(t)-\phi\left(t-\tau_{e x t}\right)\right) \cdot \frac{\tau_{e}}{\Gamma C_{t h}} .
$$


For simplification, we will neglect the time delay of the reflected waves in the feedback term for the moment. Including the above normalized feedback term in the normalized photon rate equations (which include gain saturation, spectral hole burning, carrier transport, and gain nonlinearities effects) yields:

$$
\frac{d P_{n}(t)}{d t}=\frac{\left(C_{n}(t)-C_{t r}^{n}\right)}{\tau_{p h}\left(1-C_{t r}^{n}\right)} \frac{P_{n}(t)}{\sqrt{1+P_{r}^{n}}}-\frac{P_{n}(t)}{\tau_{p h}}+\beta_{s p} C_{n}(t)+\frac{2}{\tau_{L}} \kappa_{e x t} P_{n}(t) \cos \left(\omega_{t h} \tau_{e x t}\right)
$$

and

$$
\frac{d C_{n}(t)}{d t}=\frac{I(t)}{q V_{c} C_{t h}}-\frac{\left(C_{n}(t)-C_{t r}^{n}\right)}{\tau_{e} \tau_{p h}\left(1-C_{t r}^{n}\right)} \frac{P_{n}(t)}{\sqrt{1+P_{r}^{n}}}-\frac{C_{n}(t)}{\tau_{e}}
$$

where $P_{n}(t)$ is the normalized photon density, $C_{n}(t)$ is the normalized carrier density, $\tau_{p h}$ is the photon lifetime, $\omega_{t h}$ is the angular resonance frequency for $n=n_{t h}, n_{t h}$ is the carrier density at the lasing threshold, $C_{t r}$ is the threshold carrier density, $\tau_{e}$ is the electron lifetime, $\beta_{s p}$ is the spontaneous emission factor, and $q$ is the electronic charge, and $V_{c}$ is the volume of the active region.

The above rate equations describe the laser diode's characteristics in coupled cavity. Numerical solution methods are required to solve these equations due to their extensive nonlinearity.

\subsection{Model Implementation}

\subsubsection{Symbolically Defined Laser Diode Models Including Feed- back Term}

Agilent ADS design software was used to implement the laser model based on the augmented rate equations. DC, transient, and harmonic balance simulation tools were used to analyze the laser performance. The time-domain and frequency domain simulation capabilities of the Agilent ADS software makes it a good candidate for the proposed laser mode implementation. In particular, a "rapid model prototyping" component in ADS referred to as "Symbolically Defined Device" (SDD) makes the software well-suited for exploratory work in laser model development. ADS can cover all aspect of communications system design from circuit level to system level simulation and from analog to digital systems design. Moreover, it is a suitable environment to integrate the laser model with the 
other components in the system, these include laser driving circuits and laser pre-distorter models.

A SDD describes a set of equations in terms of port currents and port voltages and automatically generates the derivatives and load information. SDDs automatically determine the relationship between equations. A SDD is represented in the circuit schematic as an $n$-port device, with up to 10 ports. The equations that specify the voltage and current of a port are defined as functions of other voltages and currents.

The $n$-port SDD is described by $n$ equations, called constitutive relationships, that relate the $n$ port currents and the $n$ port voltages. The constitutive relationships may be specified in either explicit or implicit representations. For the explicit representation, the current at port $k$ is specified as a function of port voltages, $i_{k}=f\left(v_{1}, v_{2}, \ldots ., v_{n}\right)$, and is written as $\mathrm{I}[\mathrm{a}, \mathrm{b}]$, where $a$ is the port number, and $\mathrm{b}$ indicates the order of the derivatives (e.g. 1 means $1^{\text {st }}$ derivative). The implicit representation uses an implicit relationship between any of the port currents and any of the port voltages, $f_{k}=f\left(v_{1}, v_{2}, \ldots ., i_{n}, i_{1}, i_{2}, \ldots ., i_{n}\right)$, and is written as $F[a, b]$.

A 4-port SDD, shown in Figure 3.1 on the following page, was used to implement the augmented rate equations as a combination of implicit and explicit part relationships. The drive current is represented by the current flowing into Port 1 of the SDD and is defined by the explicit function $\mathrm{I}[1,0] . P_{n}$ is represented by the current flowing into Port 2 of the SDD and is defined by the implicit function $F[2,0]$. Similarly, $C_{n}$ is represented by the current flowing into Port 3 of the SDD and is defined by the implicit function F[3,0]. The derivative, $P_{n}^{\prime}(t)$, is related to the current flowing out of Port 2 of the SDD by the explicit relation $\mathrm{F}[2,1]$. The derivative $C_{n}^{\prime}(t)$ is related to the current flowing out of Port 3 of the SDD by the explicit relation $F[3,1]$. The introduced feedback term is represented by port 4 of the SDD. The current-controlled voltage source shown by the schematic diagram in Figure 3.1 on the next page is used to de-normalize the normalized photon density into the actual intracavity photon density. This is accomplished by dividing the photons number by the normalization factor. 


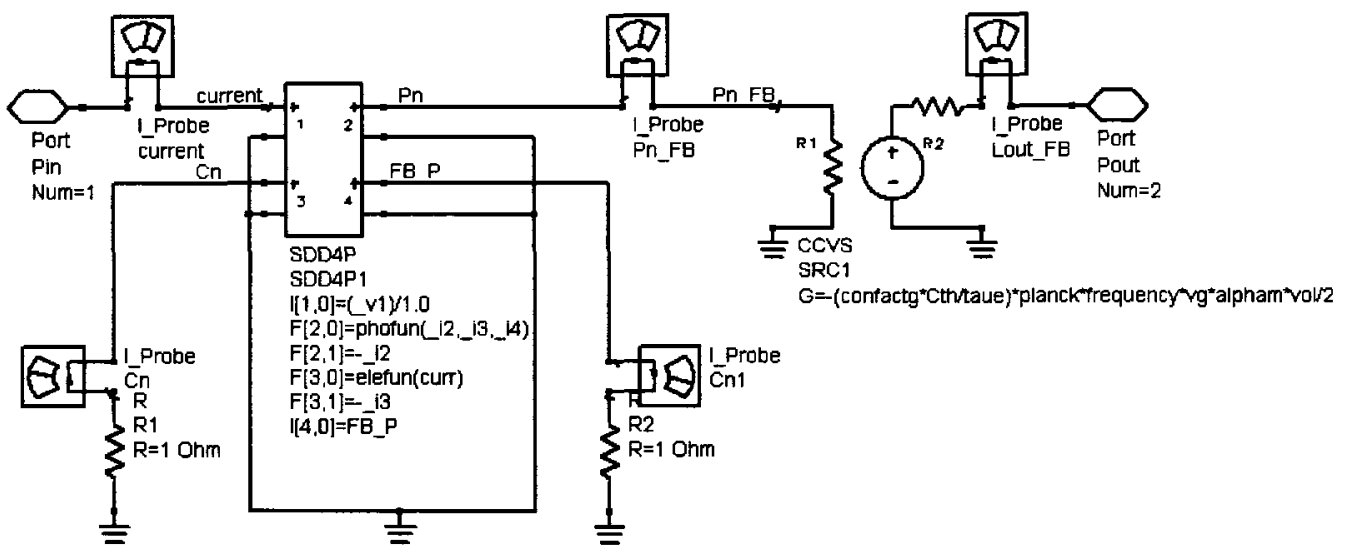

Figure 3.1: 4-port SDD laser model with external feedback. The drive current is represented by the current flowing into Port $1 . P_{n}$ is represented by the current flowing into Port 2. $C_{n}$ is represented by the current flowing into Port 3. The introduced feedback term is represented by port 4 of the SDD.

\subsubsection{Parameters Extraction}

The simulations are carried out with the parameters given in Table 3.1 on the following page. These parameters were obtained using the parameter extraction algorithm presented in [19], in which the DC and frequency response measurements data for a DFB laser diode were acquired from an optical multimeter, an optical spectrum analyzer, and a lightwave signal analyzer. The acquired data together with the laser's data sheet parameters, and the other given parameters for both laser and external cavities were used in the different mathematical relations to extract the laser diode parameters. These parameters are used in the laser rate equations that are represented by the ADS laser model in order to perform the DC, time domain, and frequency domain simulations.

\subsection{Simulation Results}

\subsubsection{Simulation of Laser Performance changes due to Feedback}

Due to the optical back-reflections the required threshold gain of the laser is changed, it can be reduced or increased depending on the phase of the reflected light, $\phi_{L}$. Slight changes in $\phi_{L}$ or the optical frequency, $\nu$, can result in considerable lasing threshold gain, 
Table 3.1: Laser parameters

\begin{tabular}{|c|c|c|}
\hline Symbol & Parameter name & value \\
\hline$L$ & Laser cavity length & $250 \mu \mathrm{m}$ \\
\hline$B_{o}$ & Radiative recombination coefficient & $1 \times 10^{-16} \mathrm{~m}^{3} \mathrm{~s}^{-1}$ \\
\hline$W$ & Laser cavity width & $2 \mu m$ \\
\hline$C_{a}$ & Auger recombination coefficient & $3 \times 10^{-41} \mathrm{~m}^{6} \mathrm{~s}^{-1}$ \\
\hline$d$ & Laser cavity thickness & $0.2 \mu m$ \\
\hline$d_{m}$ & Dipole moment & $9 \times 10^{-29} \mathrm{~m} . \mathrm{C}$ \\
\hline$n_{g}$ & Group refractive index & 4 \\
\hline$\tau_{\text {in }}$ & Dipole relaxation time & $0.1 \times 10^{-12} s$ \\
\hline$\sigma$ & Medium conductivity & $37.2 \Omega^{-1} m^{-1}$ \\
\hline$\tau_{c}$ & Electrons intraband relaxation time & $0.3 \times 10^{-12} s$ \\
\hline $\bar{n}$ & Effective mode index & 3.5 \\
\hline$\tau_{\nu}$ & Holes intraband relaxation time & $0.07 \times 10^{-12} s$ \\
\hline$\Gamma$ & Mode confinement factor & 0.3 \\
\hline$\beta_{s p}$ & Spontaneous emission factor & $1 \times 10^{-4}$ \\
\hline$\wp$ & Differential gain coefficient & $2.5 \times 10^{-20} \mathrm{~m}^{2}$ \\
\hline$L_{e x t}$ & External cavity length & $1.5 \mathrm{~m}$ \\
\hline$C_{\text {tro }}$ & Transparency carrier density & $1 \times 10^{24} m^{-3}$ \\
\hline$r_{2 e x t}$ & External cavity reflectivity & 0.20 \\
\hline$A_{n r}$ & Nonradiative recombination coefficient & $1 \times 10^{8} s^{-1}$ \\
\hline$\eta$ & Coupling coefficient & 0.75 \\
\hline
\end{tabular}


$g_{c}$, changes. FP lasers may suffer from mode hopping noise which can be avoided for single mode DFB lasers. However, for DFB lasers the external cavity may introduce additional external cavity modes, causing possible mode hopping. The optical feedback phenomenon is described by the three parameters $K_{\text {ext }}$, C, and X given by (2.6.6 on page 18), ( 2.6 .8 on page 18), and ( 2.6 .9 on page 18 ) respectively. The following results show how the laser diode performance can be affected by those three parameters.

\section{Possible emission frequencies}

Possible modes of the laser with feedback are characterized by the threshold gain and phase condition, requiring a round trip phase change $\Delta \phi_{L}=0$, or multiples of $2 \pi$. Figure 3.2 is simulation results showing the relationship between round trip phase change and optical frequency when there is no feedback $\left(r_{2 e x t}=0\right)$, weak feedback $\left(r_{2 e x t}=0.005\right)$, and relatively strong feedback $\left(r_{2 e x t}=0.025\right)$. The other laser parameters are: $1310 \mathrm{~nm}$ wavelength, $250 \mu \mathrm{m}$ cavity length, $1 \mathrm{~m}$ external cavity length, and 0.05 laser exit facet reflectivity. With no feedback, $\Delta \phi_{L}$ varies linearly with the optical frequency, yielding a single zero at the solitary laser frequency, $\nu_{t h}$. For weak feedback, $\Delta \phi_{L}$ monotonically increase with optical frequency yielding a single zero for $\Delta \phi_{L}$, hence still resulting in a single emission frequency. For relatively strong feedback, $\Delta \phi_{L}$ undergoes strong oscillations yielding multiple zeros for $\Delta \phi_{L}$; several external cavity modes around the solitary laser may also oscillate.

\section{Maximum oscillation frequency shift due to feedback}

The maximum feedback induced lasing frequency shift depends on the feedback parameter, C. For small values of $\mathrm{C}$, that is weak feedback, $\Delta \phi_{L}$ and the optical frequency, $\nu$, are monotonic functions yielding no additional modes due to the optical feedback. Figure 3.3 shows the simulated maximum oscillation frequency shift due to feedback versus laser cavity length for $1310 \mathrm{~nm}$ wavelength, 0.025 external reflectivity $r_{2 e x t}$, and 0.05 laser exit facet reflectivity $r_{2 s}$. Results show that the maximum frequency shift is a decreasing function of laser cavity length. For a cavity length of $150 \mu \mathrm{m}$, the emission frequency shifts by $101.2 \mathrm{GHZ}$, this is equivalent to $0.5789 \mathrm{~nm}$ emission wavelength shift at wavelength of $1310 \mathrm{~nm}$. 


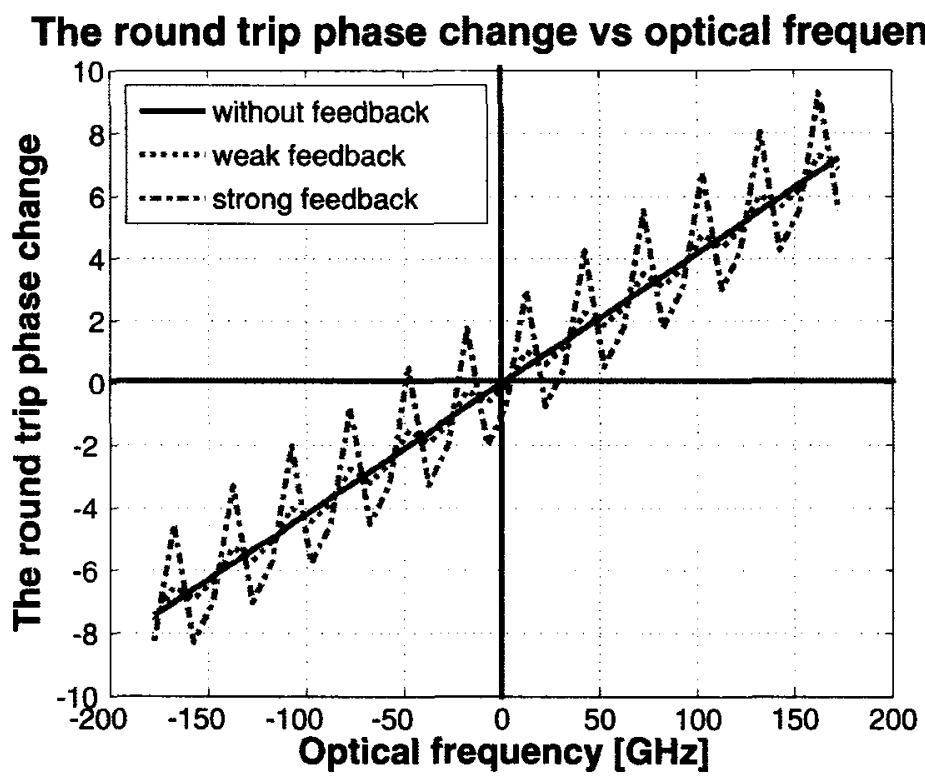

Figure 3.2: Simulated round trip phase change versus the optical frequency with no feedback $\left(r_{2 e x t}=0\right)$, weak feedback $\left(r_{2 e x t}=0.005\right)$, and relatively strong feedback $\left(r_{2 e x t}=0.025\right)$.

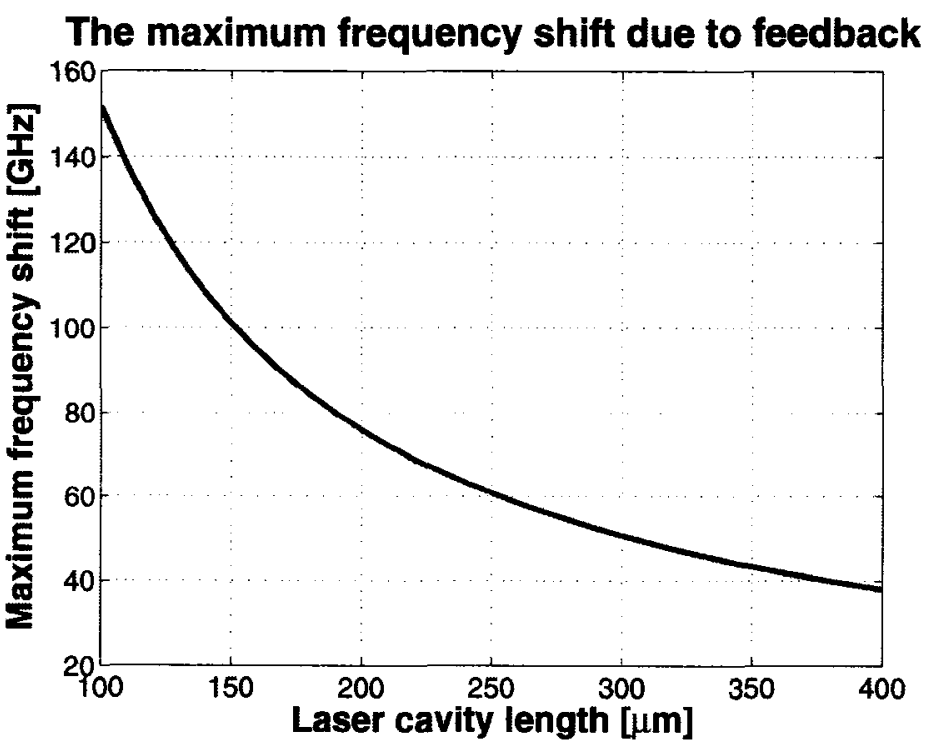

Figure 3.3: Simulated maximum oscillation frequency shift due to feedback versus laser cavity length for $1310 \mathrm{~nm}$ wavelength, 0.025 external reflectivity, and 0.05 laser exit facet reflectivity. 
Simulation results also show that the emission frequency shift is a decreasing function of the laser exit facet reflectivity, $r_{2 s}$, as depicted in Figure 3.4 for $1310 \mathrm{~nm}$ wavelength, $1 \mathrm{~m}$ external cavity length, and 0.1 external reflectivity $r_{2 e x t}$.

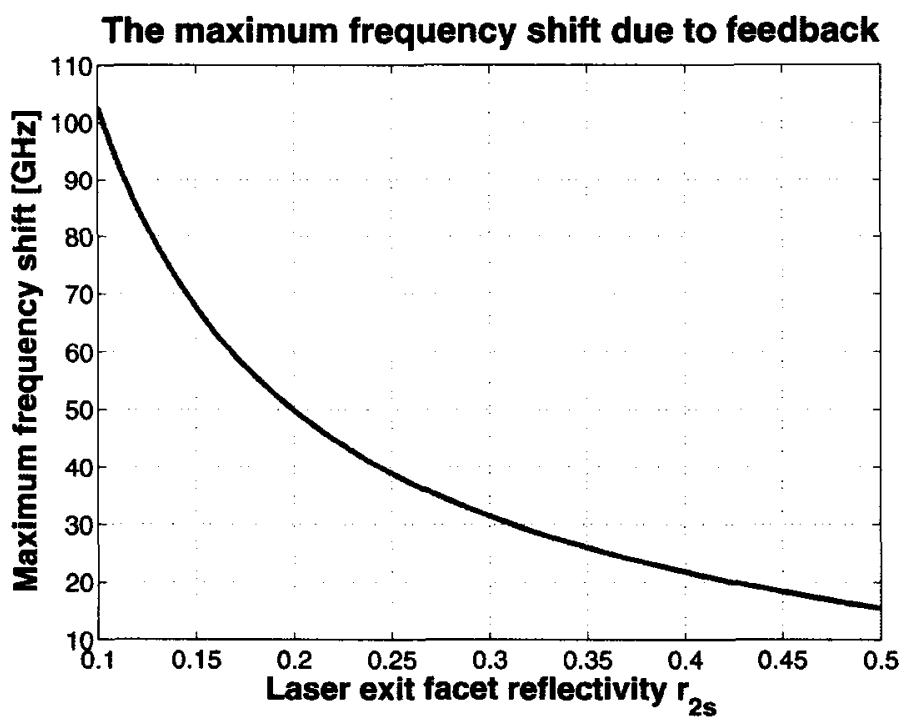

Figure 3.4: Simulated maximum frequency shift versus laser exit facet reflectivity for 1310 $\mathrm{nm}$ wavelength, $1 \mathrm{~m}$ external cavity length, and 0.1 external reflectivity.

The emission frequency shift is an increasing function of the external reflectivity, $r_{2 e x t}$, as shown in Figure 3.5 on the next page for cavity length of $350 \mu \mathrm{m}$, laser exit facet reflectivity $r_{2 s}$ of 0.32 and the same other mentioned parameters.

\section{Spectral linewidth change due to feedback}

As long as the optical back-reflections are limited to weak levels with $C<1$, the external optical feedback introduces no additional external cavity modes. Moreover depending on the phase of the reflected power, the optical feedback may improve the laser linewidth. Figure 3.6 on the following page shows the ratio of simulated spectral linewidth with feedback, $\Delta \nu$, to the spectral linewidth without feedback, $\Delta \nu_{0}$, with the feedback phase adjusted for minimum linewidth. The result shows that for a cavity length of $250 \mu \mathrm{m}$, wavelength of $1310 \mathrm{~nm}$, external cavity length of $1 \mathrm{~m}$, and laser exit facet reflectivity of 0.05 , an improvement of $\Delta \nu / \Delta \nu_{0}=0.51$ can be achieved with $\mathrm{C}=0.4$. 
The maximum frequency shift due to feedback

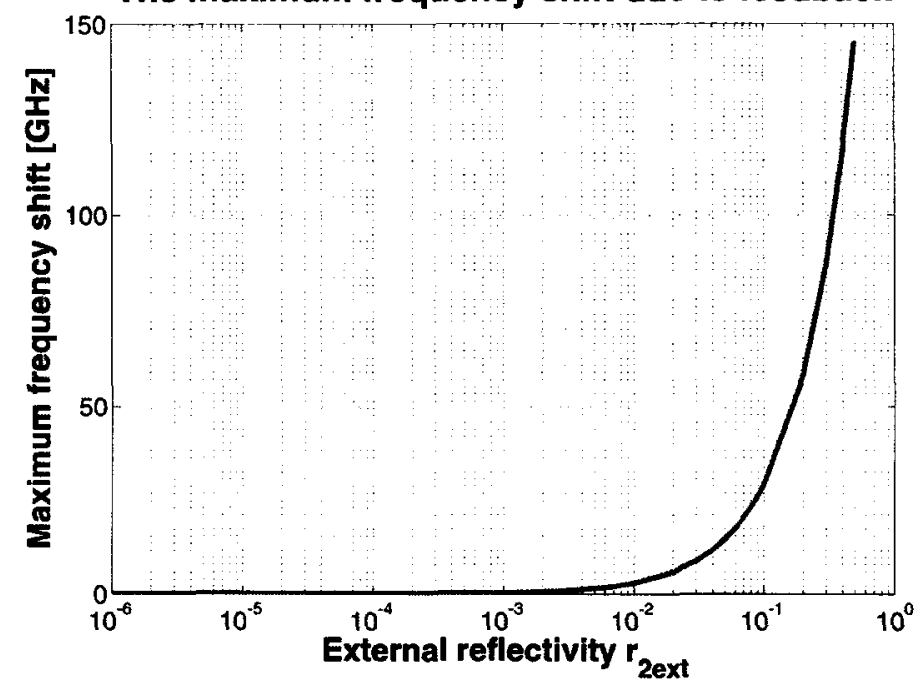

Figure 3.5: Simulated maximum oscillation frequency shift versus external reflectivity for cavity length of $350 \mu \mathrm{m}, 1 \mathrm{~m}$ external cavity length, laser exit facet reflectivity of 0.32 .

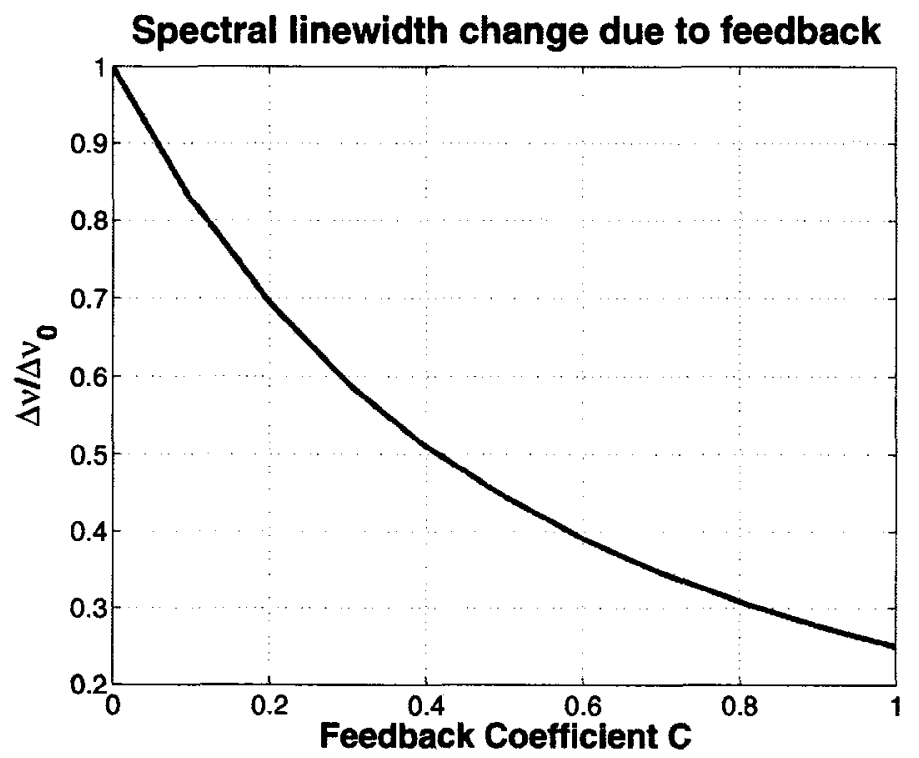

Figure 3.6: Simulated spectral linewidth with weak feedback for $1 \mathrm{~m}$ external cavity length, and 0.05 laser exit facet reflectivity; feedback phase is adjusted for minimum linewidth. 


\subsubsection{Simulation}

A threshold current value, $I_{t h}$, is required for the laser to build up a threshold gain and to start lasing. Below this threshold point, the laser emits a spontaneous radiation and acts like a light-emitting diode.

In this section the proposed model is used to predict the relationship between optical output power and diode drive current for two cases, that is with and without feedback effect. The model is used also to examine the effect of feedback on the laser diode threshold level.

A swept-parameter DC simulation was used to simulate the DC behavior of the DFB laser diode, both with and without feedback as shown in Figure 3.7 on the next page. The bias current was swept from near DC to $100 \mathrm{~mA}$, and the external reflectivity, $r_{2 e x t}$, was swept from 0.1 to 1 in 0.1 step.

The DC simulation results show that as the feedback level increases, the laser threshold current decreases as demonstrated in the optical output power versus current curve given in Figure 3.8 on page 49 [53]. Laser threshold levels at different external reflectivity values were extracted from Figure 3.8 on page 49 and plotted in Figure 3.9 on page 49. These results are confirmed through measurements as will be shown in Chapter 4 .

\subsubsection{DFB Laser Diode Frequency Response}

The proposed model was used to simulate the dynamic behavior of the laser diode and to predict the laser modulation response, modulation bandwidth, and relaxation oscillation frequency. This was performed using the ADS harmonic balance simulation capabilities.

The laser model shown in Figure 3.1 on page 41 and represented by the diode symbol in Figure 3.10 on page 50 was directly modulated with an small input sinusoid signal. The input signal modulation frequency was ranging from $0.1 \mathrm{GHz}$ to $15 \mathrm{GHz}$. The laser diode behavior was simulated both with and without the feedback effect to determine the impact of the feedback on the laser dynamic behavior. The external reflectivity was swept from 0.1 to 0.6 for a total of four levels and the external cavity length, $L_{e x t}$, was set to $2 \mathrm{~m}$.

Figure 3.11 on page 51 shows the simulated laser modulation response with and without feedback. Simulation results demonstrate that higher relaxation oscillation frequencies are obtained at higher feedback levels. An enhancement of $200 \mathrm{MHz}$ can be achieved with 0.6 external reflectivity located at $2 \mathrm{~m}$ apart from the laser exit facet. 


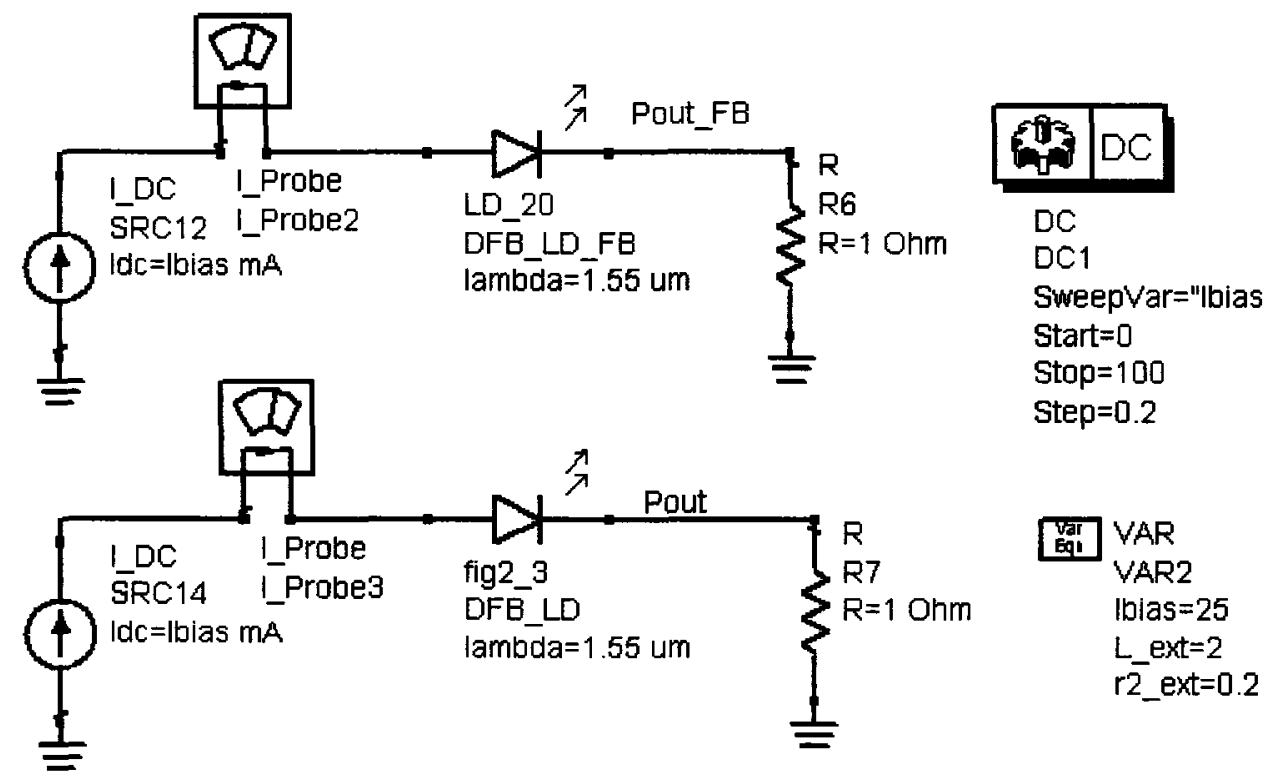

鸠 VAR

global VAR 1

$W=1.26 \mathrm{e}-6 ;$ Cavity width

$L=250 \mathrm{e}-6$; Cavity length

tauv $=0.07 \mathrm{e}-12$; Holes intraband relaxation time $\mathrm{d}=0.2 \mathrm{e}-6$; Cavity hight

$n g=4$; Group refractive index

segma $=0.372 \mathrm{e} 2$;Medium conductivity

nbar=3.5; Effective mode index

confactg $=0.3$ : Mode confinement factor

gaincoefg $=2.5 \mathrm{e}-20$;Differential gain coefficient

Ctro=1e24; Transparency carrier density

Anr $=1 \mathrm{e} 8$; Nonradiative recombination rate

$B 0=1 \mathrm{e}-16$;Radiative recombination coefficient

$\mathrm{Ca}=3 \mathrm{e}-41$;Auger recombination coefficient

betasp=1e-4;:Spontaneous emission factor

dm=9e-29;Dipole moment

\subsection{PARAMETER SWEEP}

ParamSweep

Sweep3

SweepVar="r2_ext"

Start $=0$

Stop $=1$

Step $=$

tauin $=0.1 \mathrm{e}-12$;Dipole relaxation time

tauc $=0.3 \mathrm{e}-12$;Electron intraband relaxation time

Figure 3.7: Agilent-ADS schematic diagram for the swept-parameter DC simulation setup for laser model with and without feedback. 


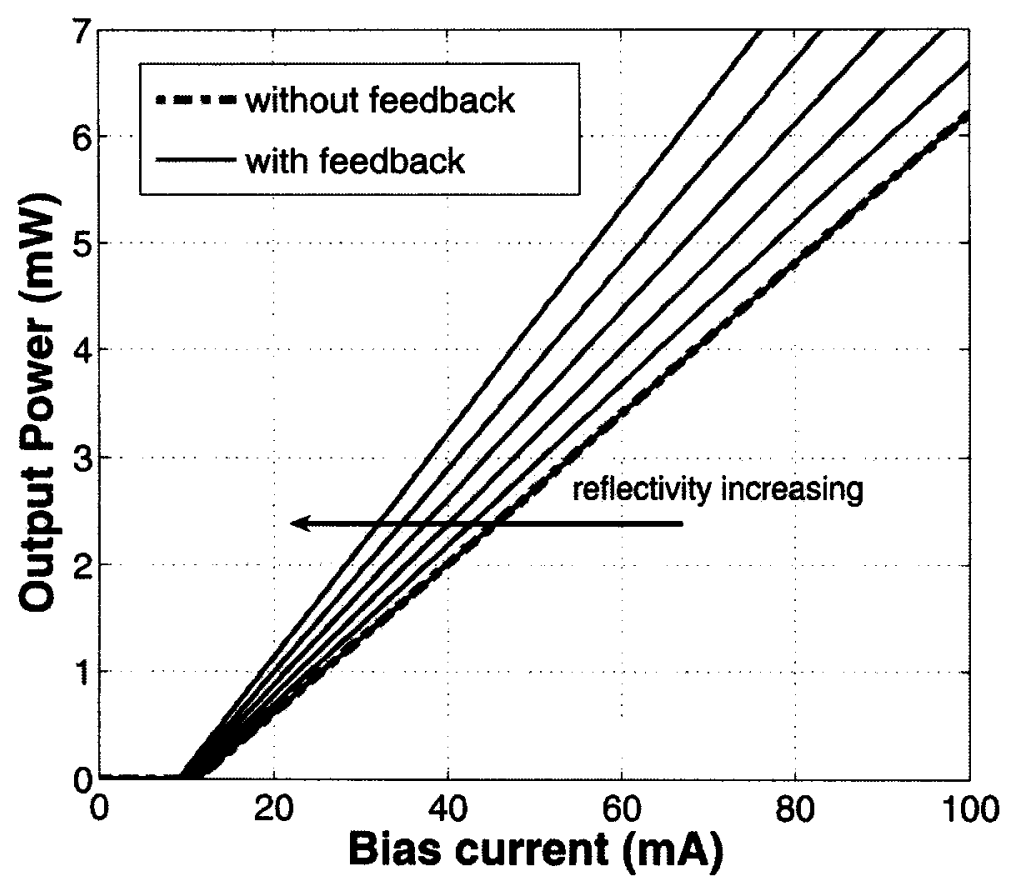

Figure 3.8: Simulated threshold current change due to back-reflection, $r_{2 e x t}$ ranges from 0.1 to 1 in $0.1 \mathrm{step}$, and $L_{e x t}=1.5 \mathrm{~m}$.

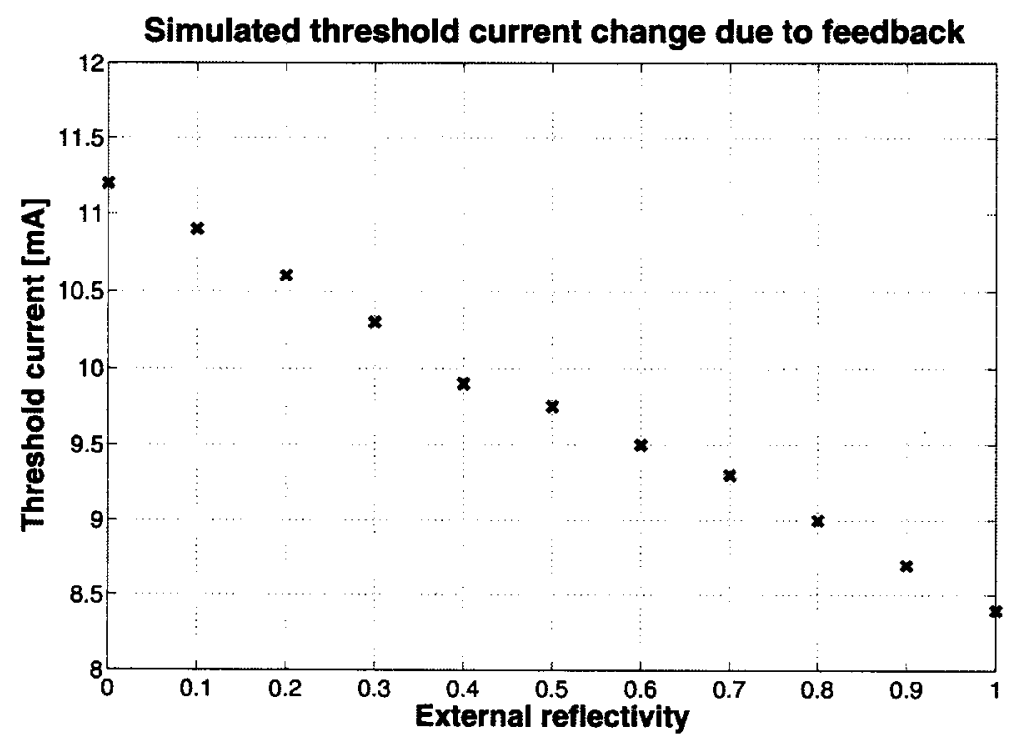

Figure 3.9: Simulated threshold current levels at different external reflectivity $r_{2 e x t}$. 

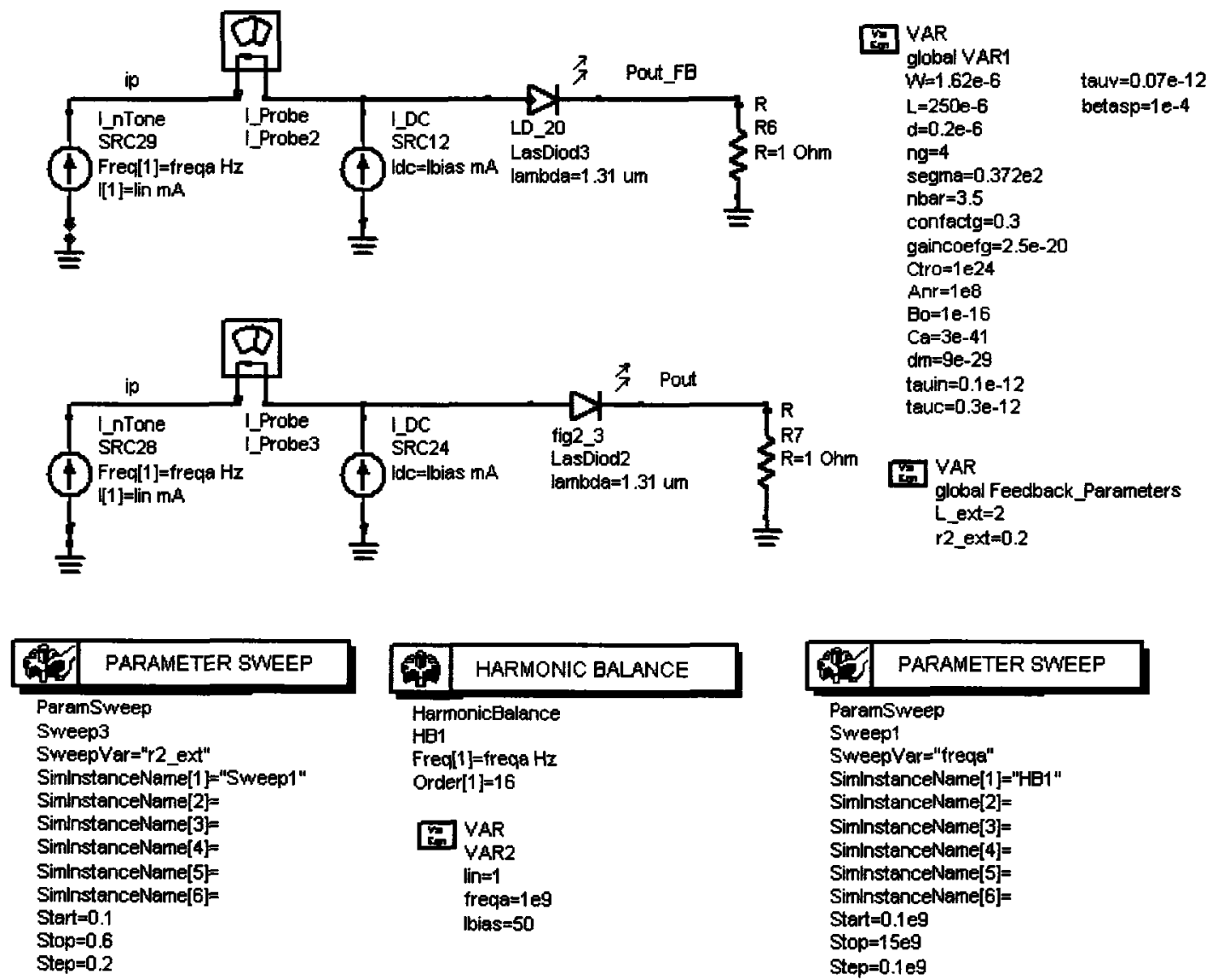

\begin{tabular}{|c|c|}
\hline 6 & HARMONIC BALANCE \\
\hline $\begin{array}{l}\text { Harm } \\
\text { HB1 } \\
\text { Freql } \\
\text { Order }\end{array}$ & $\begin{array}{l}\text { onicElance } \\
\text { 1]=freqa } \mathrm{Hz} \\
{[1]=16}\end{array}$ \\
\hline 易 & $\begin{array}{l}\text { VAR } \\
\text { VAR2 } \\
\text { lin=1 } \\
\text { freqa }=1 \mathrm{eg} \\
\text { lbias }=50\end{array}$ \\
\hline
\end{tabular}

\begin{tabular}{l}
\hline PARAMETER SWEEP \\
\hline ParanSweep \\
Sweep1 \\
SweepVar="treqg" \\
SiminstanceName[1]="HB1" \\
SimlnstanceName[2]= \\
SimnnstanceName[3]= \\
SimnstanceName[4]= \\
SimnnstanceName[5]= \\
SimnnstanceName[6]= \\
Stant=0.1e9 \\
Stop=15e9 \\
Step $=0.1 \mathrm{eg}$
\end{tabular}

Figure 3.10: Harmonic balance (HB) simulation setup for the laser model with and without external feedback.

\subsubsection{DFB Laser Diode Distortion}

Due to the importance of the laser linearity in designing optical transmitters for CATV applications, and since the dynamic range of the directly modulated lasers is mainly limited by the laser distortion levels, accurate simulations for the laser nonlinear distortions are required. The proposed model was used in the ADS HB simulation setup shown in Figure 3.12 on page 52 to simulate the laser diode distortion for both cases, that is, with and without feedback. 


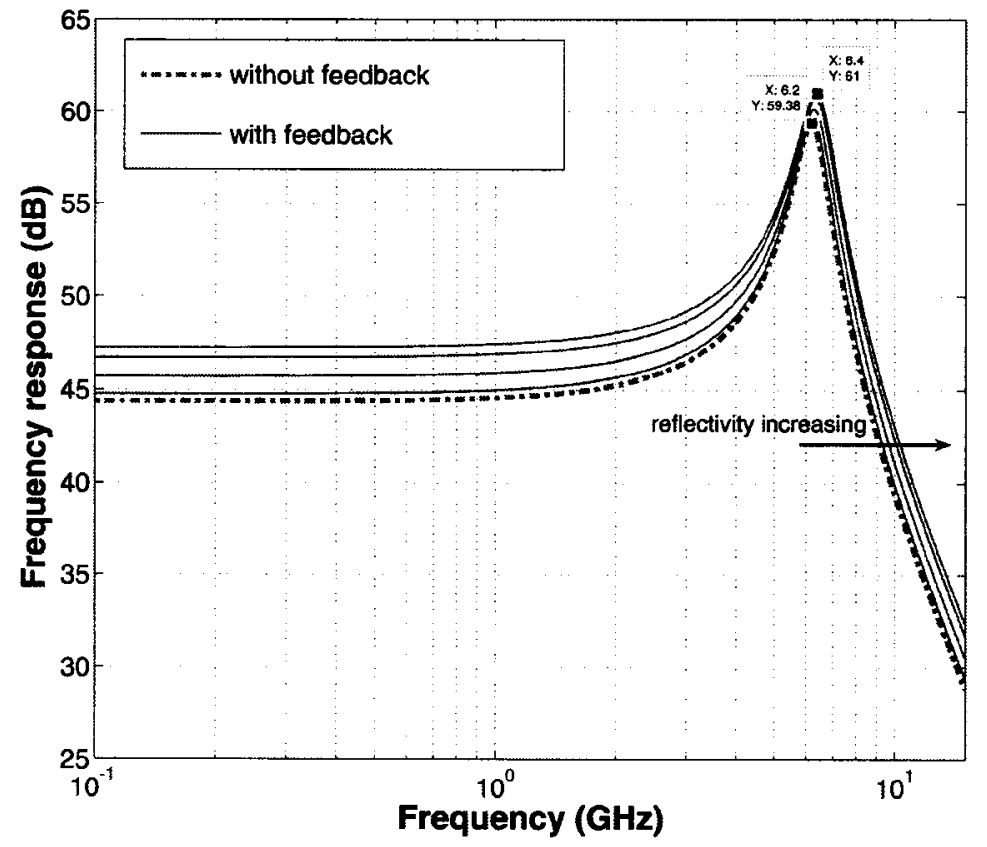

Figure 3.11: Modulation frequency response for the laser diode with and without feedback, $r_{2 e x t}$ swept from 0.1 to 0.6 for a total of four levels, and $L_{e x t}=2 \mathrm{~m}$. 


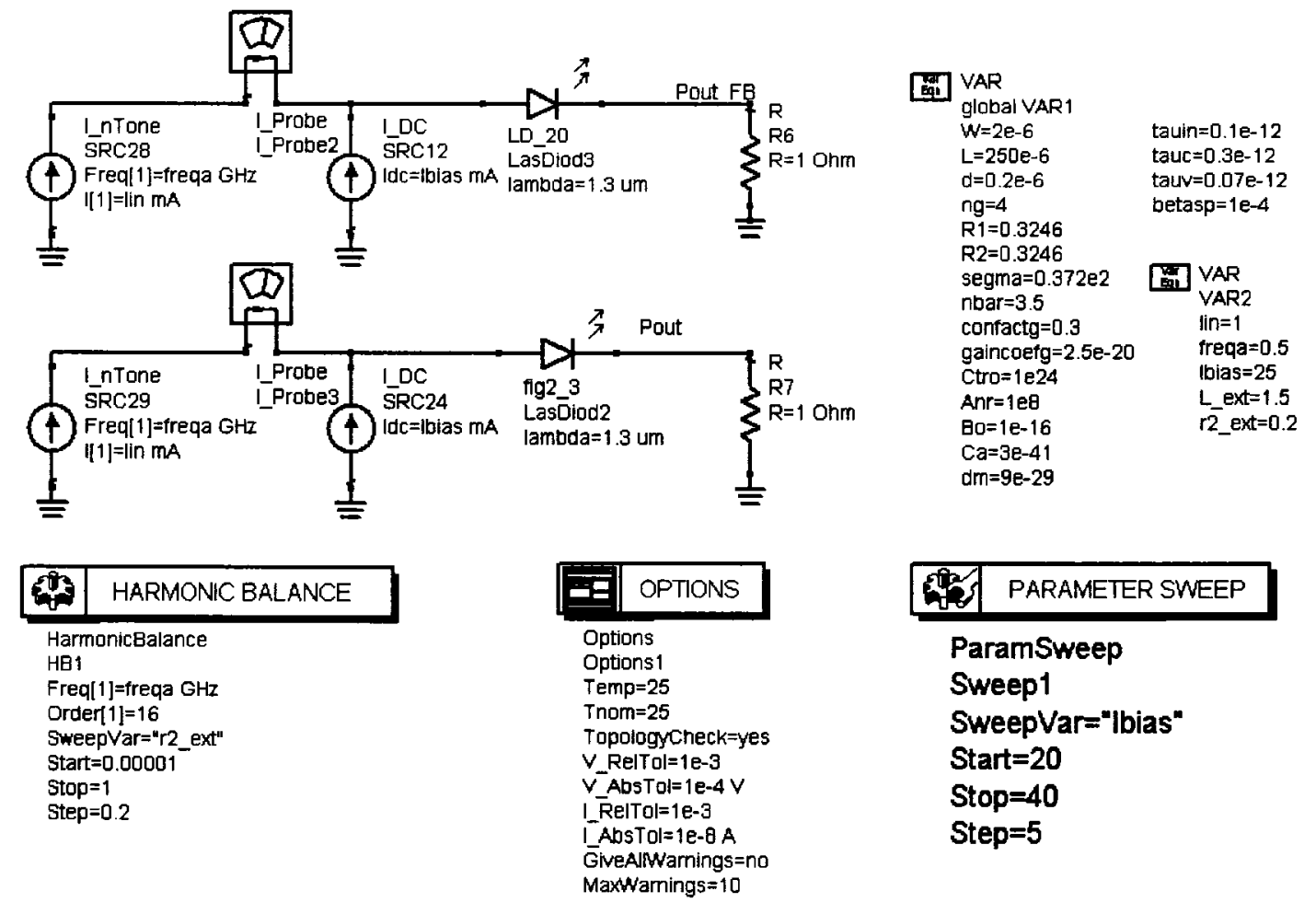

Figure 3.12: Distortion simulation setup for the laser diode with and without feedback. 
Figures 3.13 and 3.14 on the following page show the simulated second and third order harmonic distortions ( $2^{\text {nd }} H D$ and $3^{r d} H D$ ) normalized to the fundamental power versus external reflectivity, $r_{2 e x t}$, for different bias current levels respectively. The bias current was swept from 25 to $35 \mathrm{~mA}$ for a total of three levels, and the external reflectivity was swept from 0 to 1 . The other simulation parameters were: modulation frequency $f=500 \mathrm{MHz}$ , modulation signal input current $I_{i n}=1 \mathrm{~mA}$, and external cavity length $L_{\text {ext }}=1.5 \mathrm{~m}$.

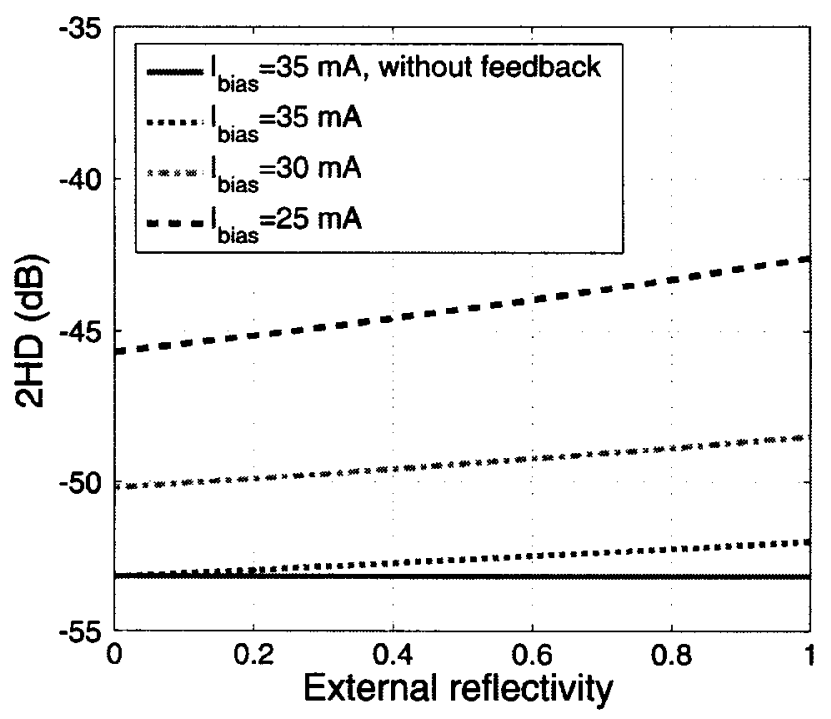

Figure 3.13: Simulated second harmonic distortion vs. external reflectivity for different bias current levels and the following parameters: $f=500 \mathrm{MHz}, I_{\text {in }}=1 \mathrm{~mA}$, and $L_{\text {ext }}=1.5 \mathrm{~m}$.

AS the results show that the $2^{\text {nd }} H D$ and the $3^{\text {rd }} H D$ are increasing functions of $r_{2 e x t}$ and are decreasing functions of $I_{b i a s}$. The simulated $2^{\text {nd }} H D$ and $3^{\text {rd }} H D$ without feedback at $I_{\text {bias }}$ of $35 \mathrm{~mA}$ were included in the figures for comparison, an increase of less than $3 \mathrm{~dB}$ is observed due to feedback at this bias current level for both $2^{\text {nd }} H D$ and $3^{\text {rd }} H D$.

Figures 3.15 on the next page and 3.16 on page 55 show the simulated $2^{\text {nd }} H D$ and $3^{r d} H D$ normalized to the fundamental power versus $I_{b i a s}$ for different $r_{2 e x t}$ levels respectively. The bias current was swept from 20 to $40 \mathrm{~mA}$, and the external reflectivity was swept from $10^{-5}$ to 0.4 for a total of three levels. The other simulation parameters were: modulation frequency $\mathrm{f}=500 \mathrm{MHz}$, modulation signal input current $I_{\text {in }}=1 \mathrm{~mA}$, and external cavity length $L_{\text {ext }}=1.5 \mathrm{~m}$. 


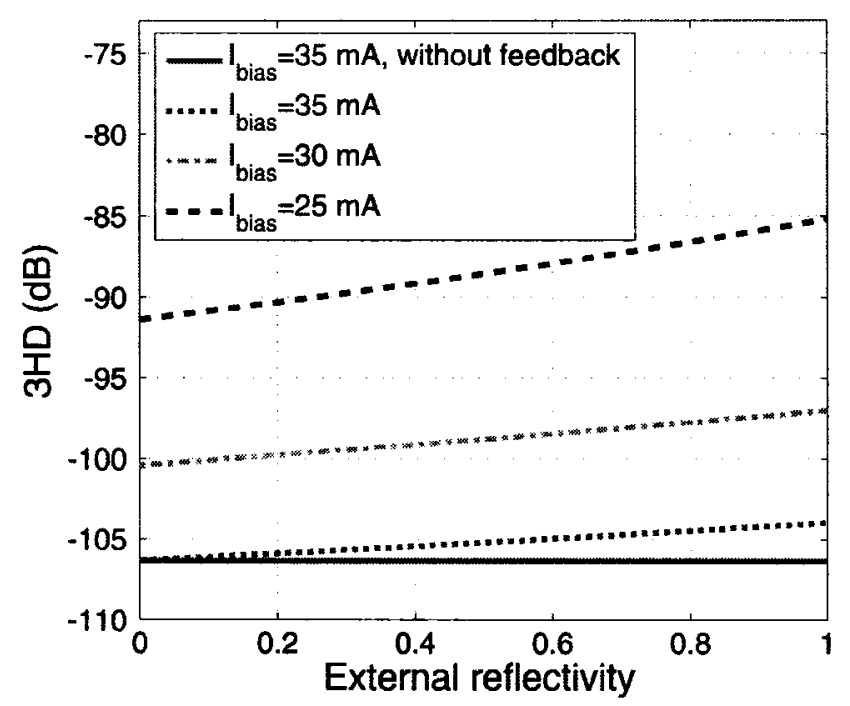

Figure 3.14: Simulated third harmonic distortion vs. external reflectivity for different bias current levels and the following parameters: $f=500 \mathrm{MHz}, I_{\text {in }}=1 \mathrm{~mA}$, and $L_{\text {ext }}=1.5 \mathrm{~m}$.

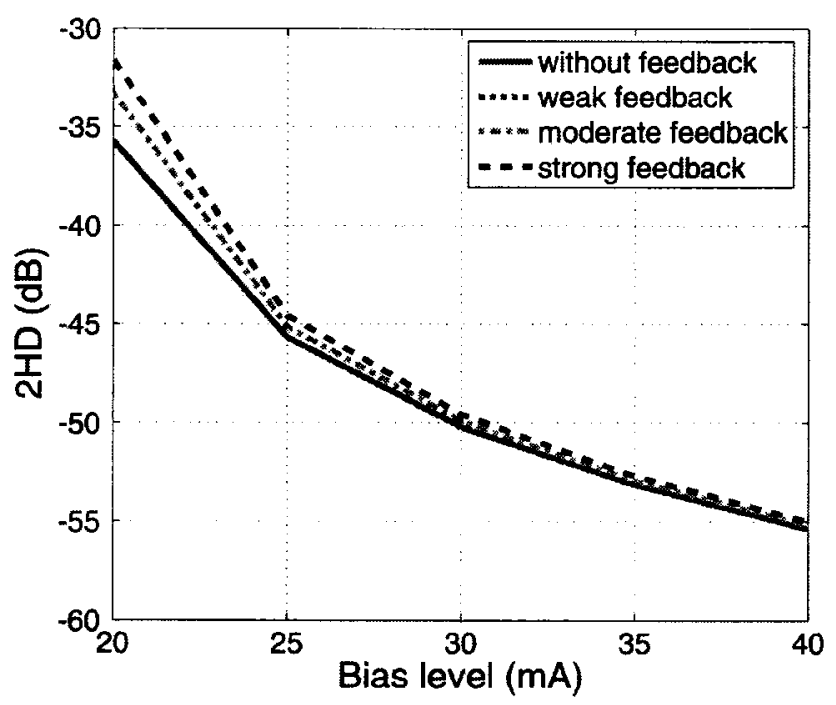

Figure 3.15: Simulated second harmonic distortion vs. bias current for different external reflectivity levels and the following parameters: $f=500 \mathrm{MHz}, I_{i n}=1 \mathrm{~mA}$, and $L_{e x t}=$ $1.5 \mathrm{~m}$. 


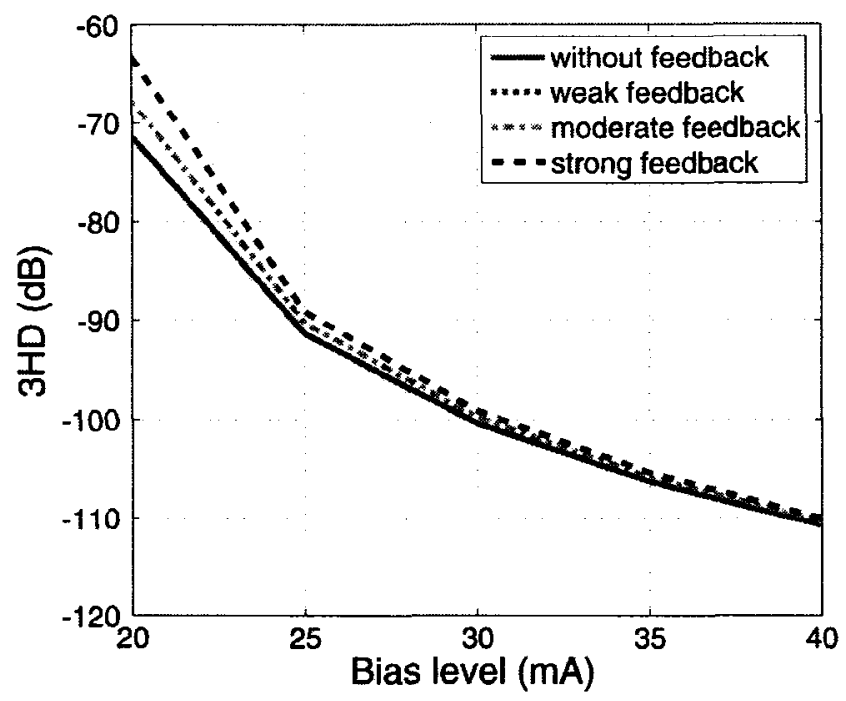

Figure 3.16: Simulated third harmonic distortion vs. bias current for different external reflectivity levels and the following parameters: $f=500 \mathrm{MHz}, I_{\text {in }}=1 \mathrm{~mA}$, and $L_{\text {ext }}=$ $1.5 \mathrm{~m}$.

The results demonstrate that the $2^{\text {nd }} H D$ and the $3^{\text {rd }} H D$ are increasing functions of $r_{2 e x t}$ and are decreasing functions of $I_{\text {bias }}$. The simulated $2^{\text {nd }} H D$ and $3^{\text {rd }} H D$ without feedback coincide with that of $10^{-5}$ external reflectivity due to the very weak reflections.

\subsection{Laser Model Including Feedback and Noise terms}

The model capability is improved by by adding the phase rate equation and including the noise terms together with the feedback terms. This will allow the model to simulate the laser diode RIN under different feedback powers.

The stochastic differential equations (3.5.1) - (3.5.3) completely define the laser system. An exact solution to those equations seems impossible; an approximation and/or numerical simulation was implemented instead. 


$$
\begin{aligned}
\frac{d P_{n}(T, t)}{d t}= & \frac{\left(C_{n}(T, t)-C_{t r}^{n}(T)\right)}{\tau_{p h}(T)\left(1-C_{t r}^{n}(T)\right)} \frac{P_{n}(T, t)}{\sqrt{1+P_{r}^{n}(T, t)}}-\frac{P_{n}(T, t)}{\tau_{p h}(T)} \\
& +\left(\beta_{s p} B_{o} \tau_{e}(T) C_{t h o} \exp \left(\frac{T-T_{r}}{T_{o}}-\frac{E_{1} q}{K_{B} T}\right)\right) C_{n}^{2}(T, t)+F_{P_{n}}(t) \\
& +\frac{2}{\tau_{L}} \kappa_{e x t} \sqrt{P_{n}(t) P_{n}\left(t-\tau_{e x t}\right)} \cos \left(\omega_{t h} \tau_{e x t}+C_{t h} V\left(\phi_{n}(t)-\phi_{n}\left(t-\tau_{e x t}\right)\right)\right) \\
\frac{d C_{n}(T, t)}{d t}= & \frac{I(t)}{q V_{c} C_{t h}(T)}-\frac{\left(C_{n}(T, t)-C_{t r}^{n}(T)\right)}{\tau_{e}(T) \tau_{p h}(T)\left(1-C_{t r}^{n}(T)\right)} \frac{P_{n}(T, t)}{\sqrt{1+P_{r}^{n}(T, t)}}-\frac{C_{n}(T, t)}{\tau_{e}(T)}+F_{C_{n}}(t) \\
\frac{d \Phi_{n}(t)}{d t}= & \frac{\beta}{2} G_{n}\left(C_{n}(T, t)-\overline{\left.C_{n}\right)+F_{\Phi_{n}}(t)}\right. \\
& -\frac{1}{\tau_{L}} \kappa_{e x t} \frac{1}{C_{t h} V} \frac{\sqrt{P_{n}\left(t-\tau_{e x t}\right)}}{\sqrt{P_{n}(t)}} \cdot \sin \left(\omega_{t h} \tau_{e x t}+C_{t h} V \cdot\left(\phi_{n}(t)-\phi_{n}\left(t-\tau_{e x t}\right)\right)\right)
\end{aligned}
$$

where $F_{P_{n}}(t), F_{C_{n}}(t)$, and $F_{\Phi_{n}}(t)$, are the normalized photon, carrier, and phase Langevin noise sources respectively.

The external power reflectivity (feedback fraction) $f_{\text {ext }}$ is given by

$$
f_{e x t}=\eta^{2} r_{2 e x t}
$$

where $\eta$ denotes the coupling efficiency between the laser and the external cavity.

The Langevin noise sources are generated using the technique presented in [17] and [19] as follows:

- Generation of three different Gaussian random variable $\mathrm{X} 1, \mathrm{X} 2$, and $\mathrm{X} 3$ with zero mean, $\langle X 1\rangle=\langle X 2\rangle=\langle X 3\rangle=0$, and unity variances, $\left\langle X 1^{2}\right\rangle=\left\langle X 2^{2}\right\rangle=\left\langle X 3^{2}\right\rangle=1$.

- Construction of Langevin photons, phase, and carrier operators as [17]:

$$
F_{P}=\sqrt{\frac{V_{P P}}{\Delta t}} X 1
$$




$$
\begin{gathered}
F_{\Phi}=\frac{1}{2(P+1)} \sqrt{\frac{V_{P P}}{\Delta t}} X 3 \\
F_{C}=\sqrt{\frac{V_{C C}+2 k V_{P C}}{\Delta t}} X 2-k F_{P}-m F_{\Phi}
\end{gathered}
$$

where $\Delta t$ is the time interval between samples, $V_{x y}$ is the variance (with $\mathrm{x}$ and $\mathrm{y}$ standing for any of $P, C$, or $\phi$ ), the parameters $k$ and $m$ are defined as:

$$
\begin{gathered}
k=-\frac{V_{P C}}{V_{P P}} \\
m=-\frac{V_{C \Phi}}{V_{\Phi \Phi}}=2 k(P+1)
\end{gathered}
$$

The new set of rate equations including feedback and noise terms are implemented in ADS as shown in Figure 3.17.

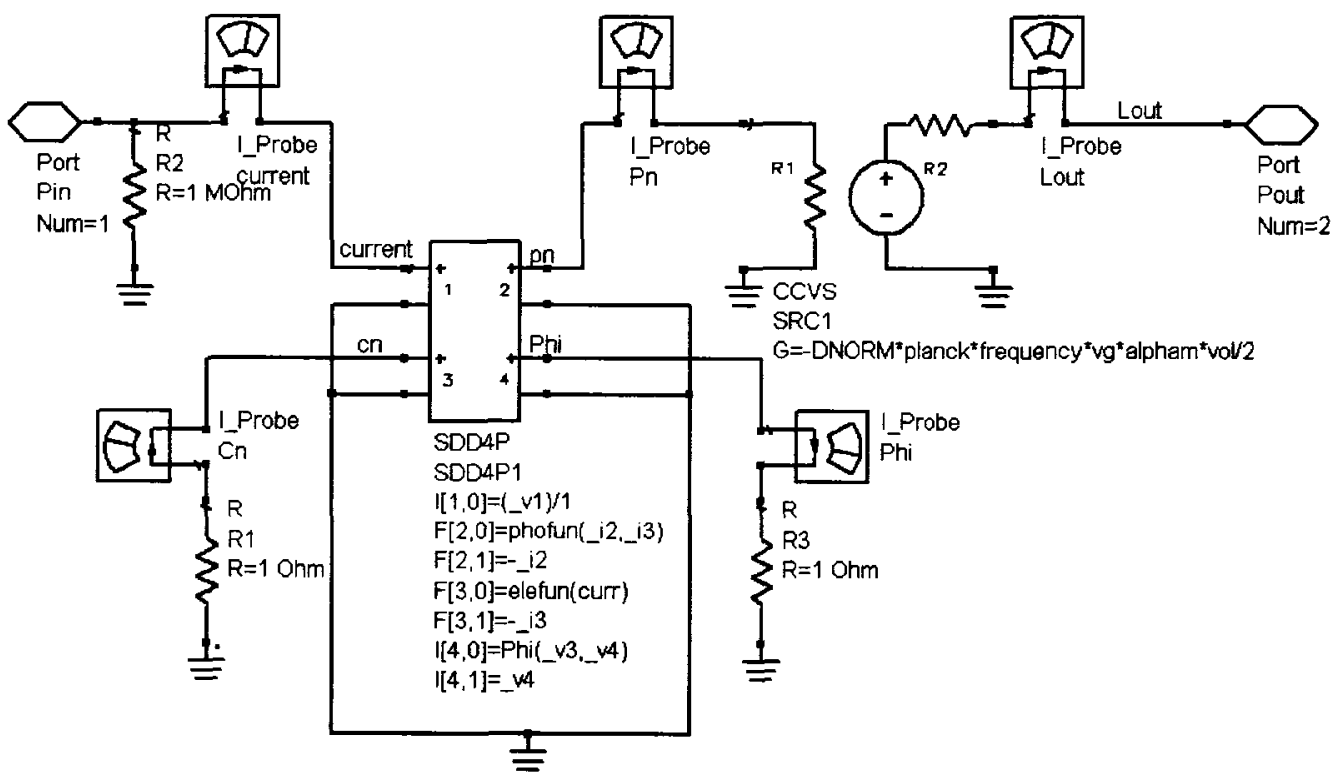

Figure 3.17: ADS laser model including feedback terms and noise operators. 
The amplitude of the noise terms were adjusted in order to get the model to converge, our main concern was to examine the laser noise behavior at different feedback powers as well as to identify the noise peak around the relaxation oscillation rather than the accurate noise amplitude itself.

\subsubsection{Relative Intensity Noise Calculation}

The RIN is defined as the ratio of the mean-square optical intensity noise to the square of the average optical power, it can be calculated using:

$$
R I N=\frac{\overline{(P(t)-\overline{P(t)})^{2}}}{\overline{P(t)}^{2}}
$$

where $P(t)$ is the laser output power, and $\overline{P(t)}$ is the laser mean power.

\subsubsection{Laser output}

Figure 3.18 on the following page shows the time domain output, that is optical power versus time with and without the noise operators. The fluctuations of the output power around the steady state value after the transient time are contributed to the Langevin noise sources that have been added to the laser rate equations. The fluctuations are less visible during the transient time due to the relative level of the transient output and the noise.

\subsubsection{Modulation Frequency Response Simulation}

The laser model was used to predict the modulation frequency response, where the DFB laser model was directly modulated with an input signal frequency ranging from $100 \mathrm{M} \mathrm{Hz}$ to $20 \mathrm{GHz}$ with $0 \mathrm{dBm}$ input signal power. Figure 3.19 on page 60 shows the simulated relaxation frequency at $6.7 \mathrm{GHz}$.

\subsubsection{RIN Simulation}

The RIN was calculated by taking an FFT of the steady state time domain data; the effect of the transients on the simulations was avoided by running the simulation beyond 

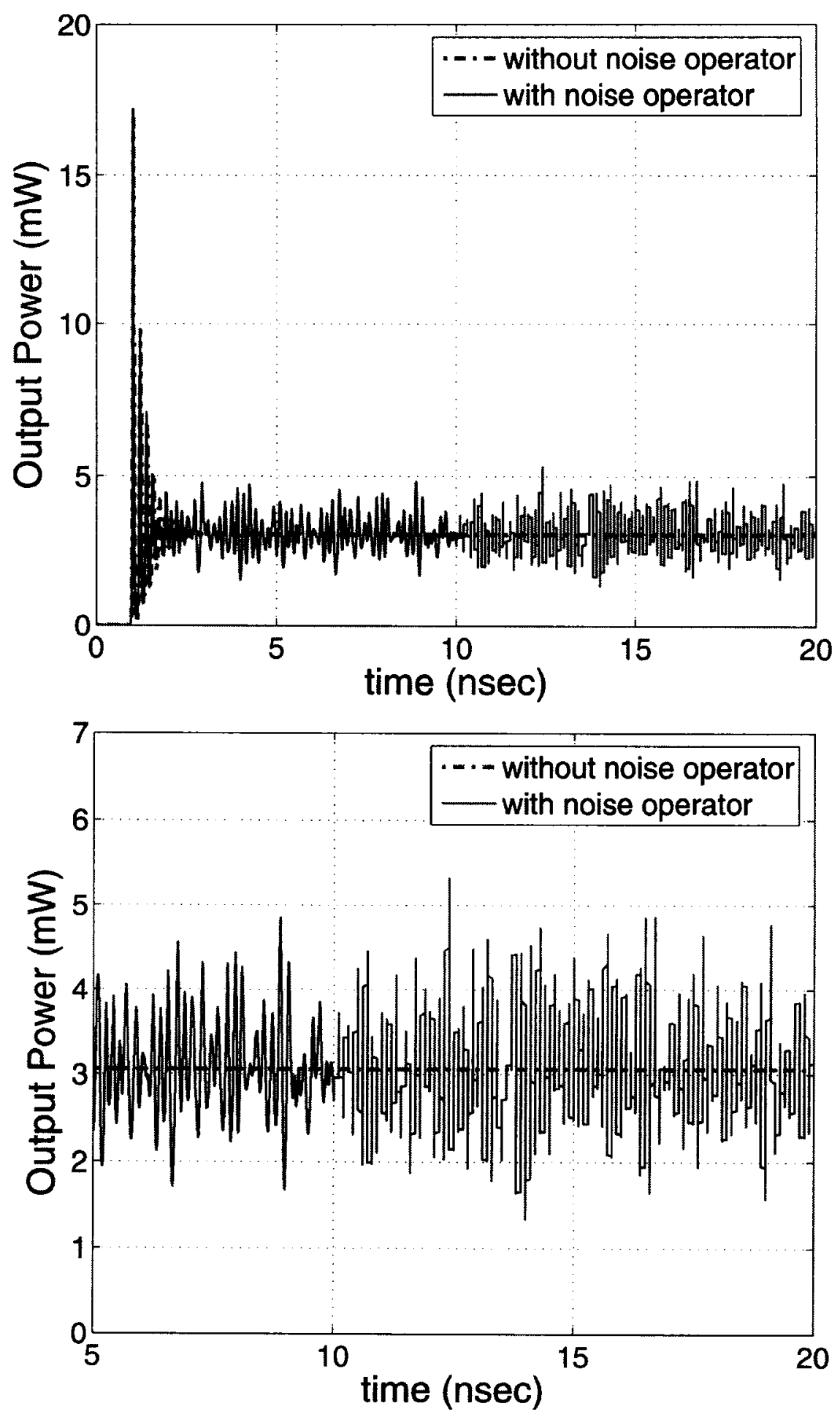

Figure 3.18: Time domain laser model's output with and without noise sources. 


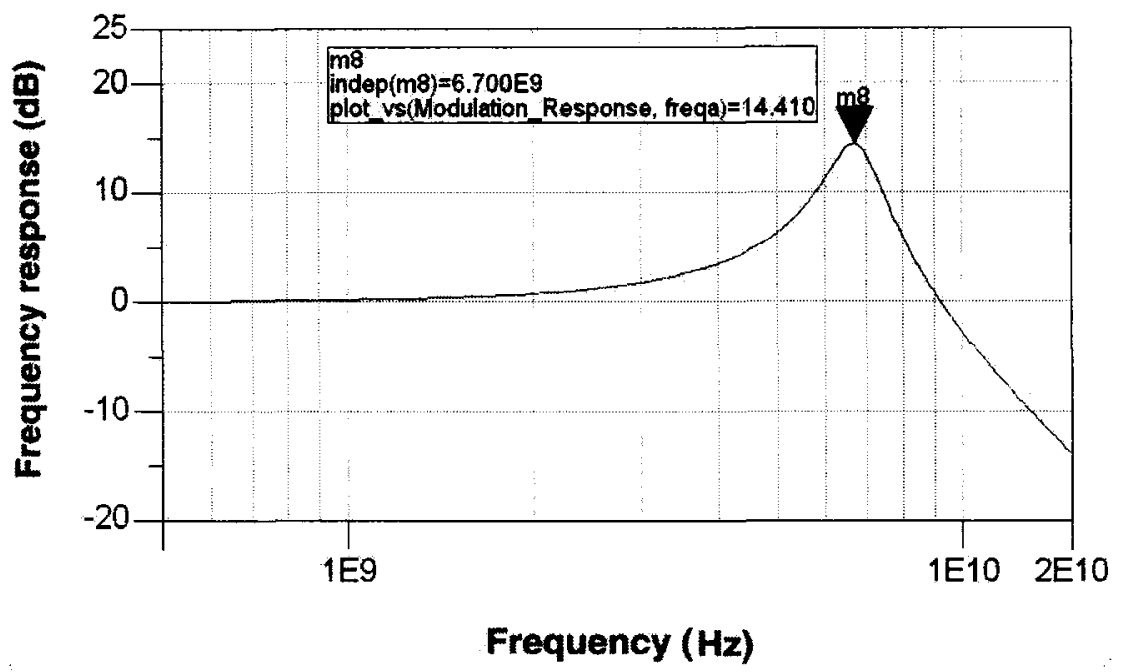

Figure 3.19: Simulated modulation frequency response .

25 nsec. A short time step of $\Delta t=10$ psec was used to cover a frequency spectrum of up to $50 \mathrm{GHz}$. The simulation was extended to up to $250 \mathrm{nsec}$ hence requiring around 22,500 integration steps. Figure 3.20 shows the simulated RIN for a DFB laser operating at the same condition as in Figure 3.19. As expected, the RIN exhibited a peak around the relaxation frequency that is $6.7 \mathrm{GHz}$.

\section{RIN versus bias current for fixed unintended optical back-reflection}

Figure 3.21 on the following page shows the simulated RIN as a function of the bias current $I_{\text {bias }}$ ranging from $12 \mathrm{~mA}$ to $40 \mathrm{~mA}$ for a fixed feedback level of $-45 \mathrm{~dB}$. The result shows that the RIN decreases exponentially as the $I_{\text {bias }}$ increases. This is in good agreement with the results previously presented in [12] and [17].

\section{RIN versus external reflectivity}

Figure 3.22 on page 62 shows the simulated RIN as a function of the external reflectivity, $r_{2 e x}$, for a fixed bias current level of $15 \mathrm{~mA}$. The RIN slightly increases as the external reflectivity increases until a certain feedback level is exceeded $(-30 \mathrm{~dB}$ in this case). A dramatic increase in the RIN occurs beyond that point due to the transition to feedback Regime IV (coherence collapse regime). The simulation results are again in good agreement 


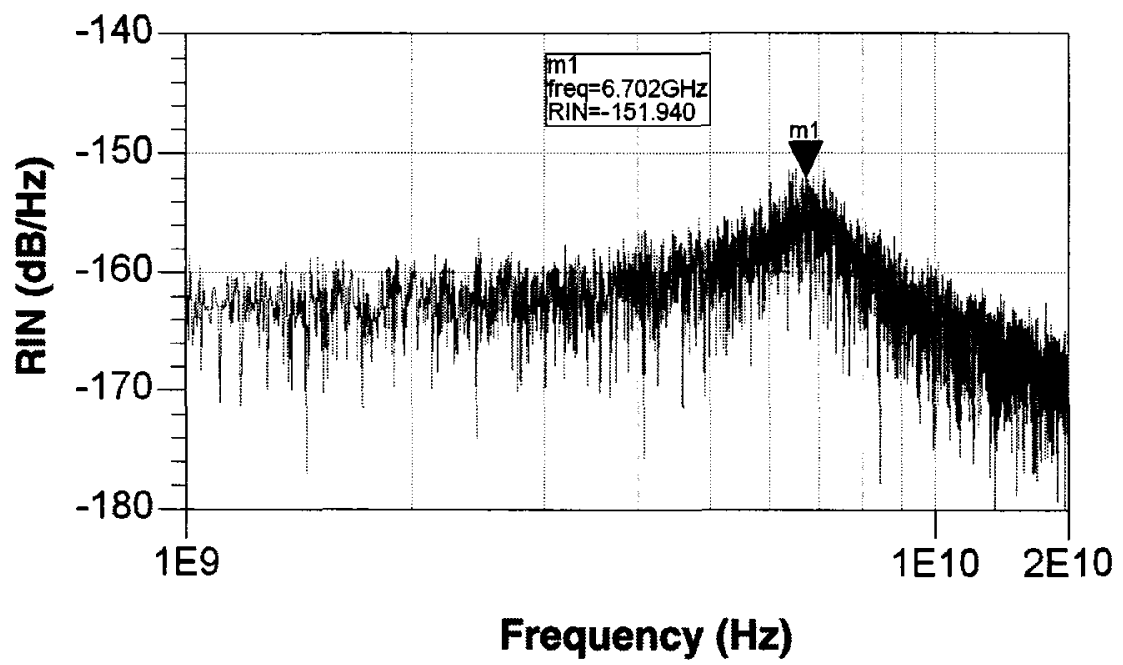

Figure 3.20: Simulated RIN of a DFB laser diode.

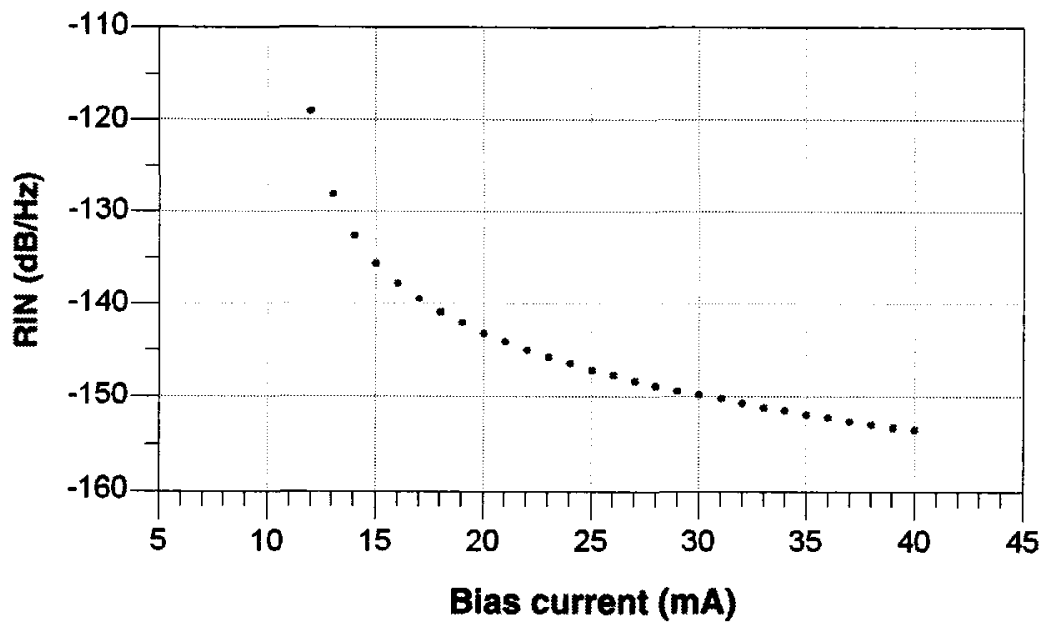

Figure 3.21: Simulated RIN as a function of bias current for a fixed feedback level of $-45 d B$. 
with the results presented in [39] and [10].

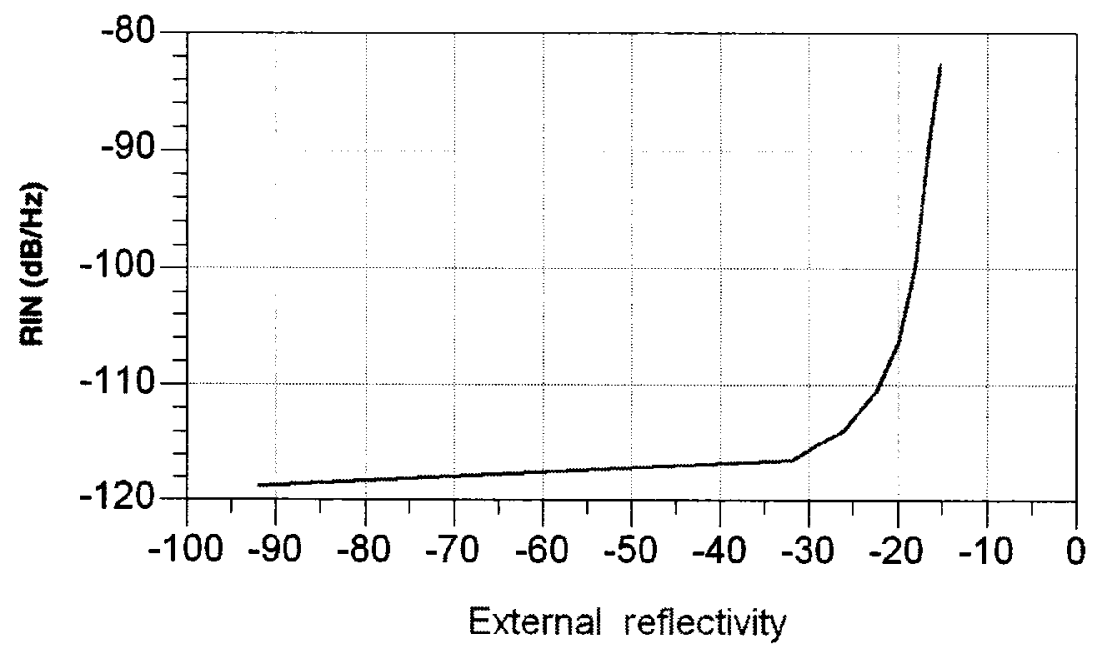

Figure 3.22: Simulated RIN versus external reflectivity.

The results presented in this chapter will next be experimentally verified in Chapter 4 . 


\subsection{Summary}

In this chapter, the simulation of DFB laser's various parameters in existence of optical feedback was presented. The feedback terms for the proposed laser rate equations were derived and normalized. A laser model was implemented in ADS using the proposed rate equations in order to simulate various laser behaviors in existence of feedback. The laser's DC characteristics, noise, and linearity were simulated using various ADS setups. The DC simulation results showed that the laser diode's threshold current level was decreased due to feedback. Modifications to the laser's modulation response and linearity were observed through harmonic balance simulations. The model was improved by including the noise terms to the rate equations and then the laser's intensity noise was simulated in a transient simulation setup. RIN simulation results matched those found in the literature. 


\section{Chapter 4}

\section{Experimental Work and Model Verification}

\subsection{Introduction}

In this chapter, we discuss the experimental setup and demonstrate the results of the simulation work. The influence of the optical back-reflections on the laser DC characteristics, threshold current changes, linearity, and noise are all examined and analyzed.

\subsection{Measurements}

In order to determine the power required for a fiber-optic link, the measurement of the output optical power should be performed and analyzed for different feedback scenarios. This section shows how the feedback power can modify the DC characteristics of the laser diode and decreases the threshold current level. The model is also verified by comparing the measured to the simulation results.

\subsubsection{Threshold Current Measurements Setup}

Two single mode DFB laser diodes with different wavelengths were used in the experimental setup shown in Figure 4.1 on the following page. DFB laser diodes were mounted on the THORLABS TCLDM9 $5.6 \mathrm{~mm} / 9 \mathrm{~mm}$ laser diode mount. The ILX lightwave LDC-3724B laser diode controller was used to control the temperature and drive the laser. The emission wavelengths for the two DFB laser diodes were $1310 \mathrm{~nm}$ and $1550 \mathrm{~nm}$. The DFB laser 
diode output was launched to the $\mathrm{T}$ port of the OZ Optics Fiber Optic Beam Splitter (FOBS) and divided into two parts. Half of the power was coupled to the ILX Lightwave OMM-6810B optical power and wavelength meter to measure the output power. The other half of the power was sent to the OZ Optics fiber optic reflector through the $\mathrm{OZ}$ Optics variable attenuator to achieve the desired back reflection level. The Agilent $86140 \mathrm{~B}$ Optical Spectrum Analyzer (OSA) was connected to the $\mathrm{R}$ port of the FOBS to monitor the reflected power. The feedback ratio was defined as the ratio of the reflected power to the laser output power without feedback. Fiber optic cables were used in the setup to avoid spurious reflections from interconnection points.

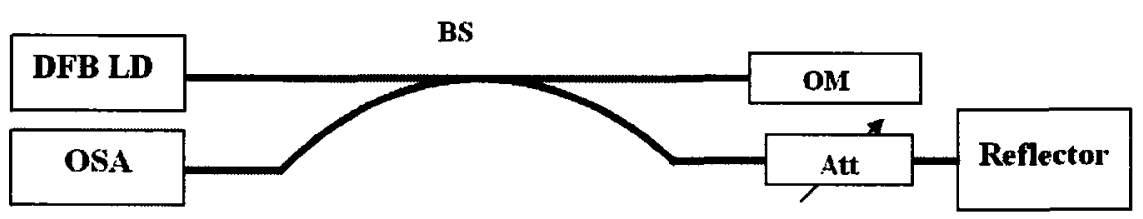

BS........Beam Splitter OSA....... Optical Spectrum Analyzer
OM......Optical Multimeter Att......... Variable attenuator

Figure 4.1: Experiment setup for DC measurements.

Figure 4.2 on the next page shows the setup used to measure the change in threshold current due to optical back-reflections. A brief description for each component is given in Appendix A.

\subsubsection{Threshold Current Results and Model Verification}

In order to obtain the optical power, the bias current was swept from 1 to $40 \mathrm{~mA}$. Figure 4.3 and Figure 4.4 on page 67 show the measured optical power versus the biasing current for the $1550 \mathrm{~nm}$ DFB laser operating without feedback, and with $-14 \mathrm{~dB}$ feedback. Figure 4.5 shows same results obtained using the $1310 \mathrm{~nm}$ DFB laser diode instead. We can conclude 


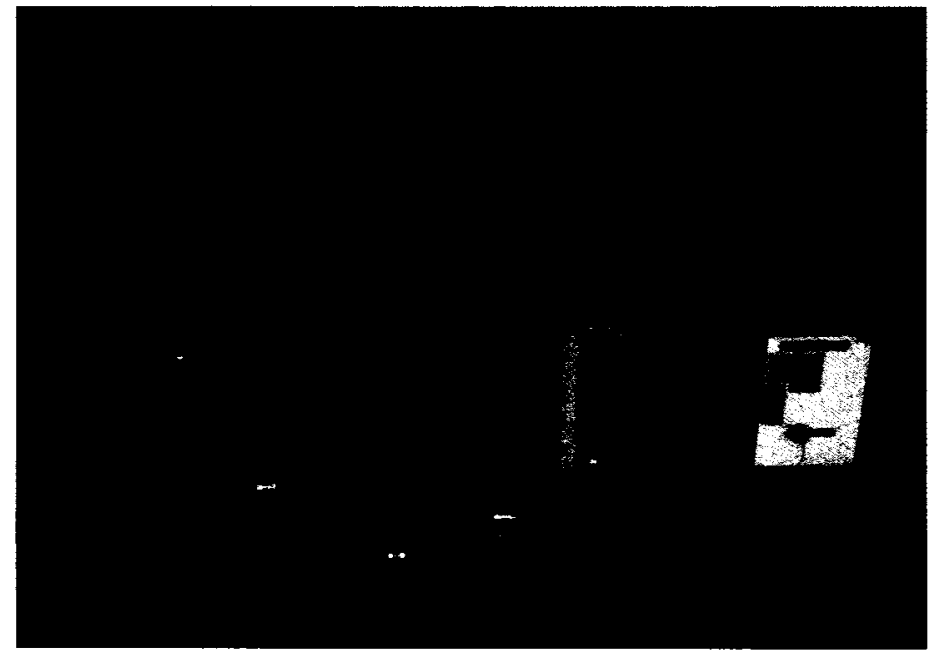

Figure 4.2: DC measurements experiment setup. Front right to left: the fiber optic reflector, the variable attenuator, the polarization controller, the fiber optic beam splitter, and the laser diode on the mount. Back right to left: the optical spectrum analyzer, the fiber optic power wavehead, the optical power and wavelength meter (top), and the laser diode controller (bottom).

from these results that the DFB laser diode threshold current was reduced due to the reflected power, which agrees with the simulated results.

Figure 4.6 on page 68 plots of the simulated threshold current versus the optical backreflection coefficient. Some measured data points were also included for comparison purposes. The power measurements accuracy for the used optical power meter is 0.05 . The results show a good agreement between simulation and measurements, the absolute error values for all measured points lie within a maximum allowable error level of 0.05 of the corresponding simulated values.

\subsubsection{DFB Laser Diode Spectra Measurement Setup and Results}

Figure 4.7 on page 69 shows the experimental setup used to examine the DFB laser diode spectra for different feedback levels. The single mode DFB laser diode was mounted on the THORLABS TCLDM9 $5.6 \mathrm{~mm} / 9 \mathrm{~mm}$ Laser diode Mount, and it was temperature controlled and biased by the ILX lightwave LDC-3724B laser diode controller. The emission wavelength for the DFB laser diode was $1550 \mathrm{~nm}$. The DFB laser diode output is sent 


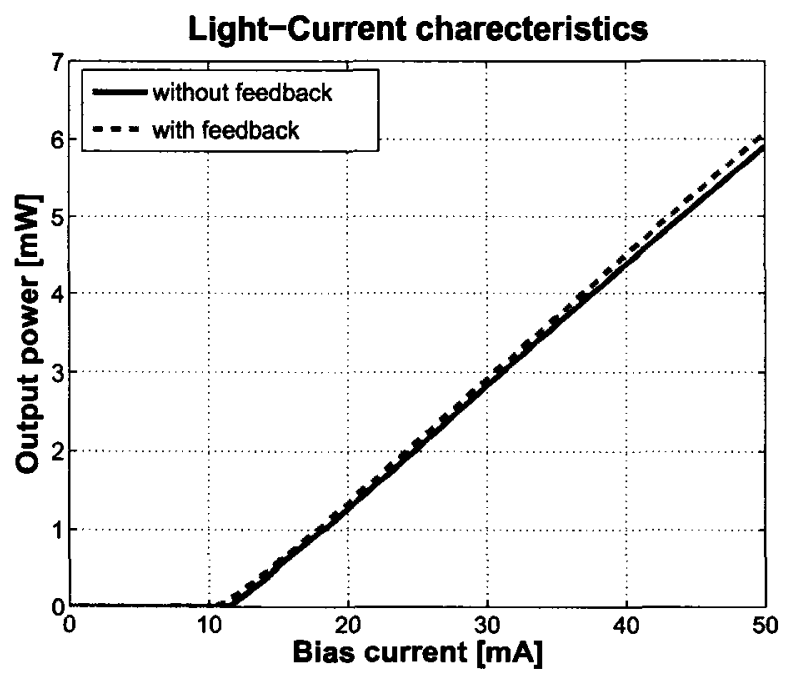

Figure 4.3: P-I curve for $1550 \mathrm{~nm}$ DFB laser diode without feedback and with $-14 \mathrm{~dB}$ feedback ratio.

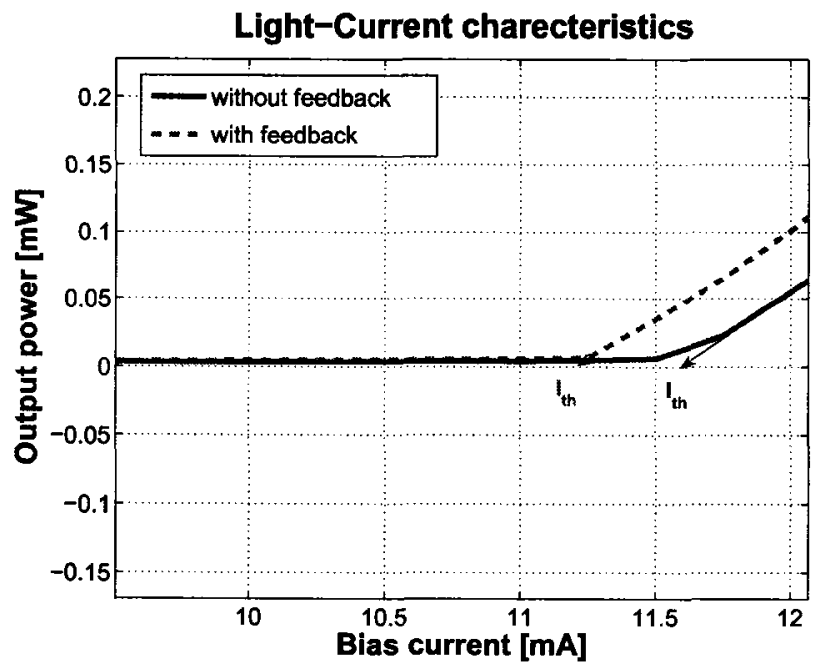

Figure 4.4: Threshold current change for $1550 \mathrm{~nm}$ DFB laser diode with -14 dB feedback ratio compared to the same laser diode without feedback. 


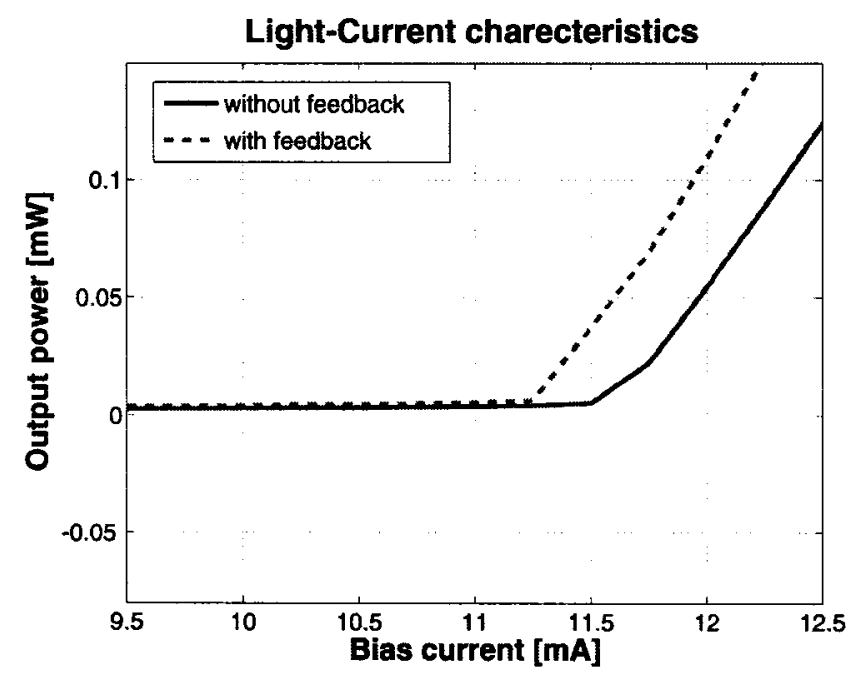

Figure 4.5: Threshold current change for $1310 \mathrm{~nm}$ DFB laser diode with -14 dB feedback ratio compared to the same laser diode without feedback.

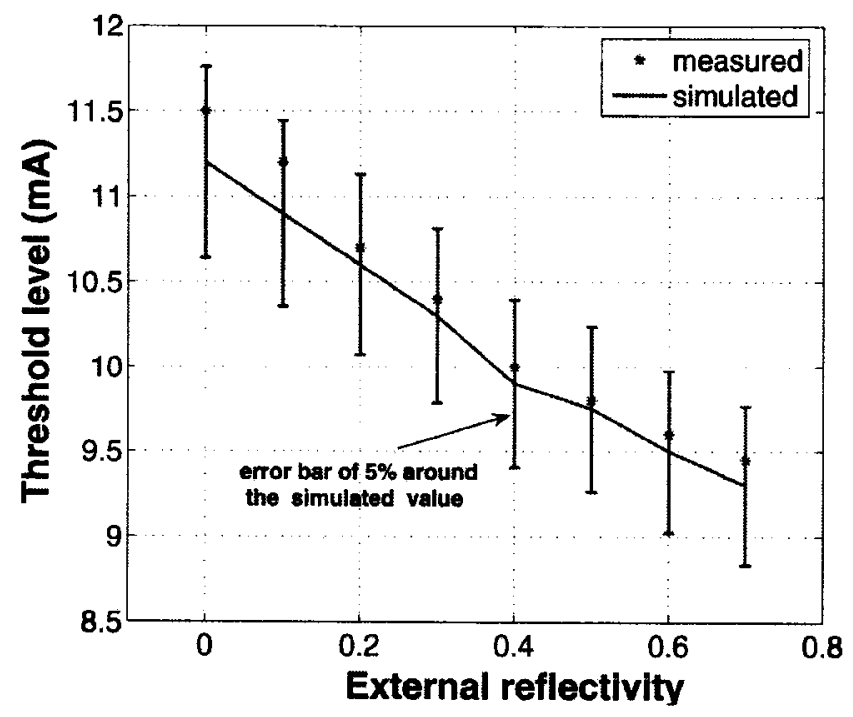

Figure 4.6: Measured and simulated threshold current change due to optical back-reflection. 
to the $\mathrm{T}$ port of the FOBS, where it was divided. One half of the power was coupled to the Agilent $86140 \mathrm{~B}$ OSA, which was used to examine the spectral characteristics of the laser diode output, while the other half was sent to the OZ Optics Fiber optic reflector to achieve the desired back reflection level. The feedback level was controlled by the $\mathrm{OZ}$ Optics variable attenuator. The OZ Optics Polarization controller was used to guarantee that the power reflected back into the laser had the same polarization as the emitted power. The reflected power through the R port of the FOBS was measured using the ILX Lightwave OMM-6810B Optical Power and Wavelength Meter. A brief description of the different components used in the measurements is included in Appendix A.

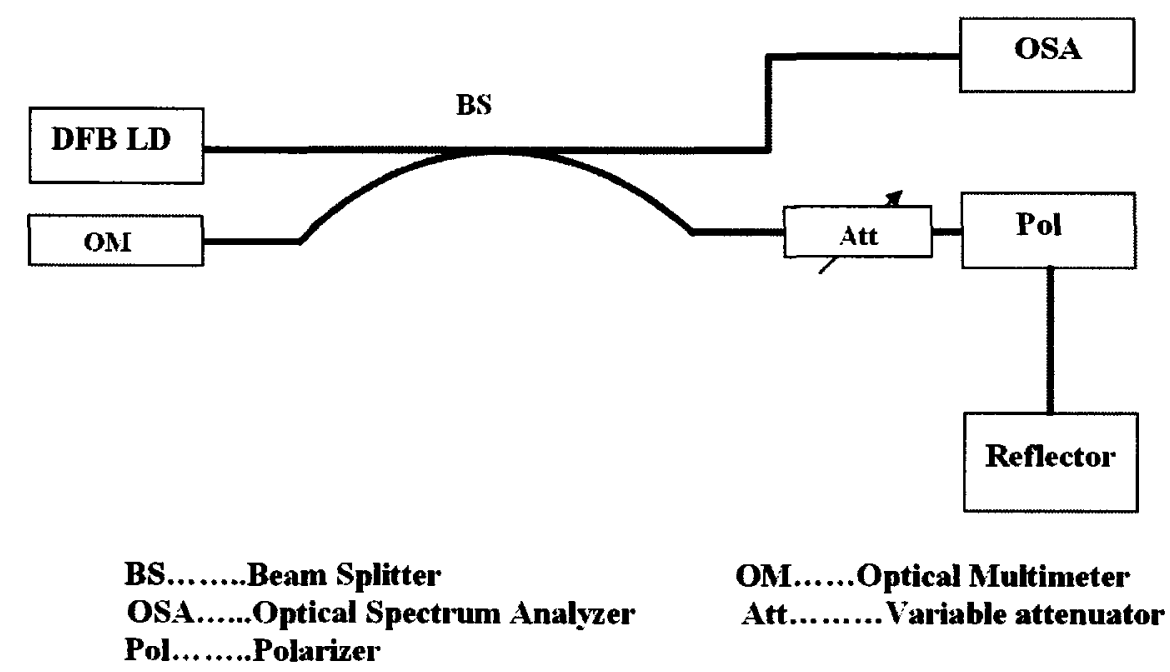

Figure 4.7: DFB laser diode spectra experiment setup.

Figure 4.8 on the next page shows the spectra of the $1310 \mathrm{~nm}$ DFB laser diode with different feedback levels. Figure 4.8(a) shows the spectrum of the free running DFB laser diode biased at $12 \mathrm{~mA}$ without any induced feedback. In this case, the DFB laser diode operates in a single longitudinal mode. The spectrum still shows a single mode operation under $-40 \mathrm{~dB}$ optical reflection as shown in Figure 4.8(b). As the feedback level was increased to $-15 \mathrm{~dB}$, the laser became unstable and the DFB laser diode spectrum was broadened due to its operation in the coherence collapse regime as shown in Figure 4.8(c). The feedback level was further increased to $-10 \mathrm{~dB}$ to drive the DFB laser diode to operate 
in the external cavity operation regime (Regime V) with a single mode operation as shown in Figure 4.8(d) [54].
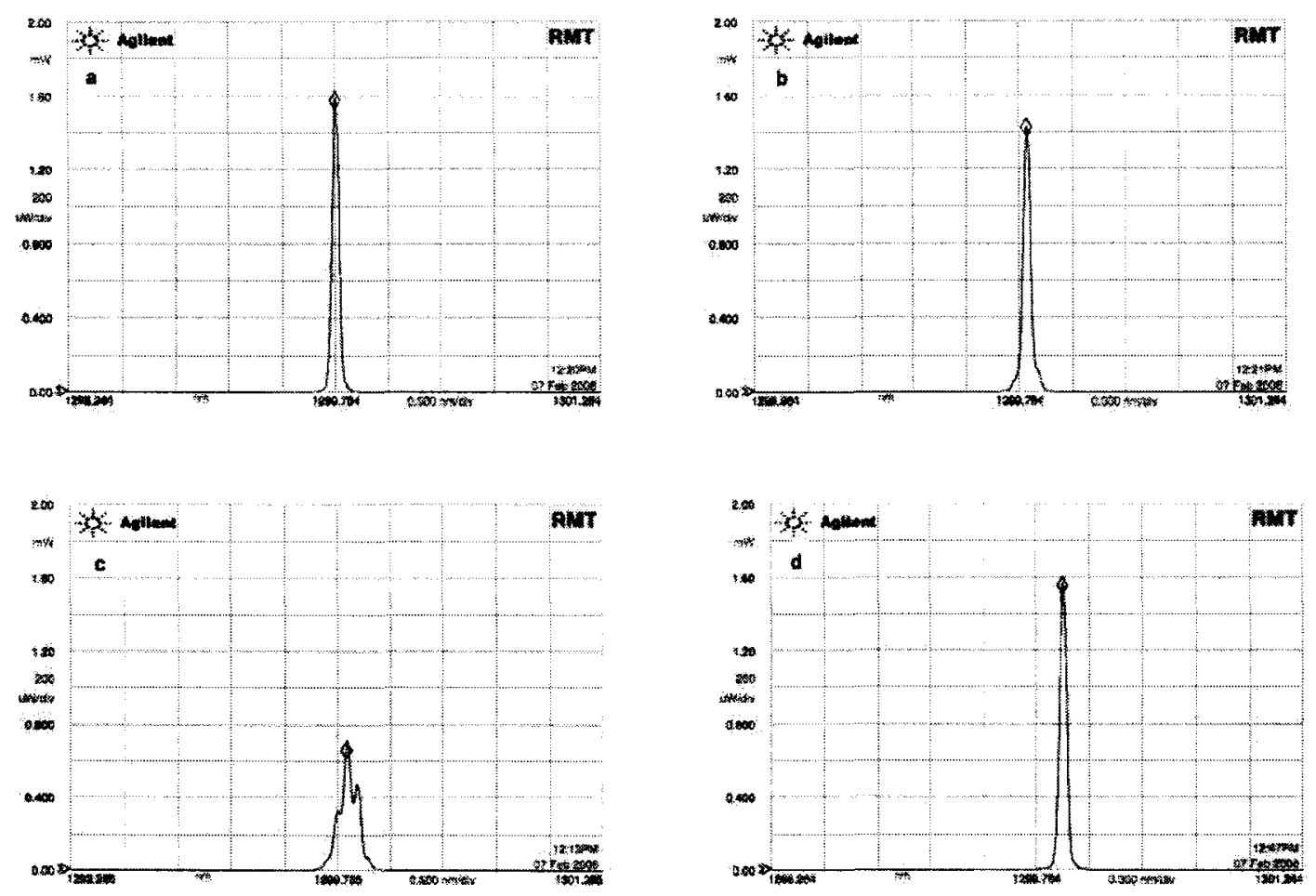

Figure 4.8: Optical spectra of the $1310 \mathrm{~nm}$ DFB laser diode biased at $12 \mathrm{~mA}$ and with $\begin{array}{lllll}\text { different optical feedback powers: } & \text { a) }-45 \mathrm{~dB} & \text { b) }-40 \mathrm{~dB} & \text { c) }-15 \mathrm{~dB} & \text { d) }-10\end{array}$ $\mathrm{dB}$.

\subsection{Noise and Linearity Measurements}

The Agilent $71400 \mathrm{C}$ lightwave signal analyzer shown in Figure 4.9 on the following page is a tool to measure important laser diode characteristics such as signal strength, modulation bandwidth, signal distortion, and noise. The Agilent 71400C lightwave signal analyzer can be used in conjunction with the Agilent 11980A fiber-optic interferometer to measure the laser diode linewidth. 


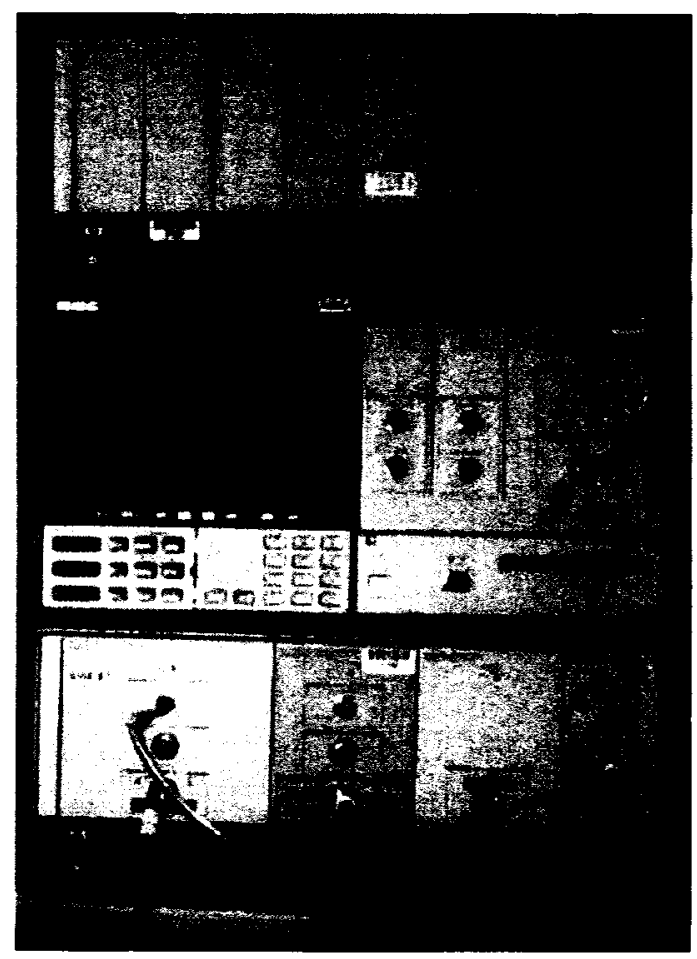

Figure 4.9: Agilent 71400C lightwave signal analyzer. 


\subsubsection{RIN Measurements Experimental Setup}

The Agilent 71400C lightwave signal analyzer with a RIN measurement module (a special software routine) was used for the laser RIN measurements. The RIN module is mainly capable of measuring two quantities, $R I N_{S Y S}$ and $R I N_{\text {laser. }} R I N_{S Y S}$ measures the total system noise taking into account the laser intensity noise, shot, and the thermal noise of the instrument. $R I N_{\text {laser }}$ is equivalent to the ratio of the laser noise to the average power preluding the shot and thermal noise terms.

Figure 4.10 on the next page shows the experimental setup used in the measurements. The DFB laser diode was mounted on the THORLABS TCLDM9 $5.6 \mathrm{~mm} / 9 \mathrm{~mm}$ laser diode mount, and it was temperature controlled and biased by the ILX lightwave LDC$3724 B$ laser diode controller. The emission wavelength for the DFB laser diode was $1310 \mathrm{~nm}$. The DFB laser diode output is sent to the T port of the FOBS, where it was split into two halves. The first half of the power was coupled to the Agilent $71400 C$ lightwave signal analyzer to measure the RIN. The measured RIN was then displayed on a 70004A display unit and connected through a GPIB (IEEE488.2 interface) to a personal computer for acquiring screen images and controlling trace data using the Agilent N1031A BenchLink lightwave software. The remaining half of the power was directed to the OZ Optics fiber optic reflector to achieve the desired back reflection level. The feedback level was controlled by the OZ Optics variable attenuator. The OZ Optics Polarization controller was used to guarantee that the power reflected back into the laser had the same polarization as the emitted power. The reflected power though the $\mathrm{R}$ port of the FOBS is measured using the ILX Lightwave OMM-6810B Optical Power and Wavelength Meter.

\subsubsection{RIN Measurement Results and Model Verification RIN versus bias current}

Figures 4.11 - 4.15 on page 75 show the measured average relative intensity noise for $1300 \mathrm{~nm}$ DFB at $1 \mathrm{GHz}$ frequency offset. The DFB laser was operated at five different bias current levels, $25,30,35,40$, and $60 \mathrm{~mA}$. The measured unintended back-reflection that attributed to the connectors was $-45 \mathrm{~dB}$.

The RIN exhibits a peak at the laser's relaxation oscillation frequency, this peak shifts 


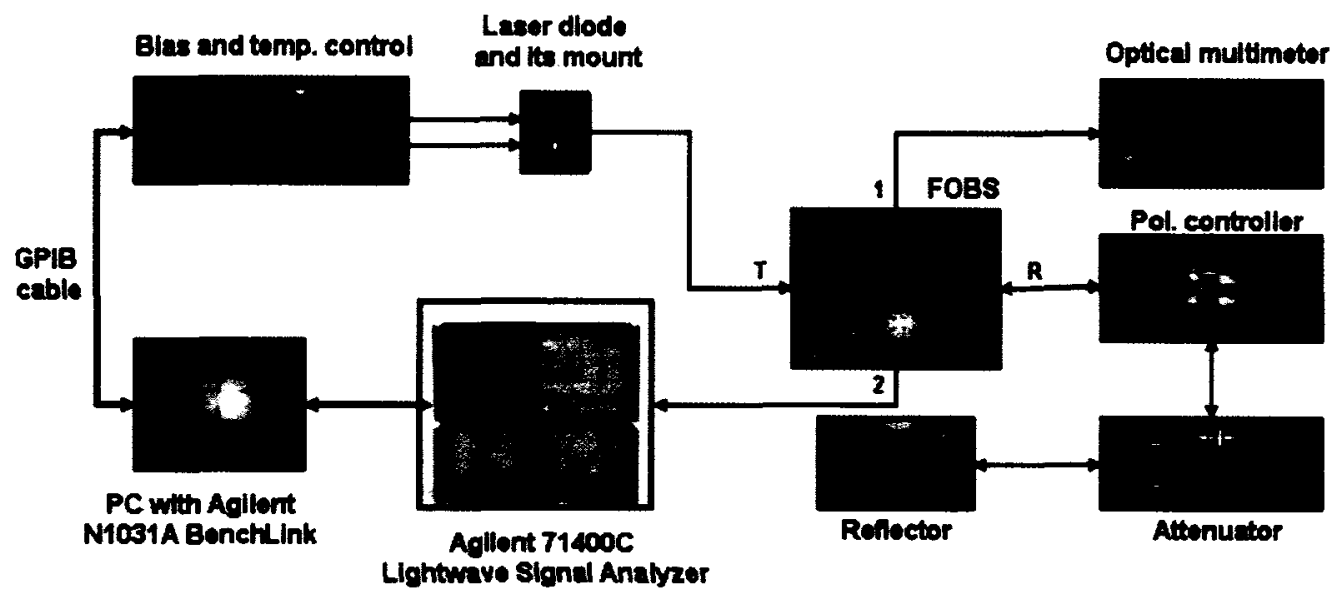

Figure 4.10: Relative intensity noise measurement experimental setup.

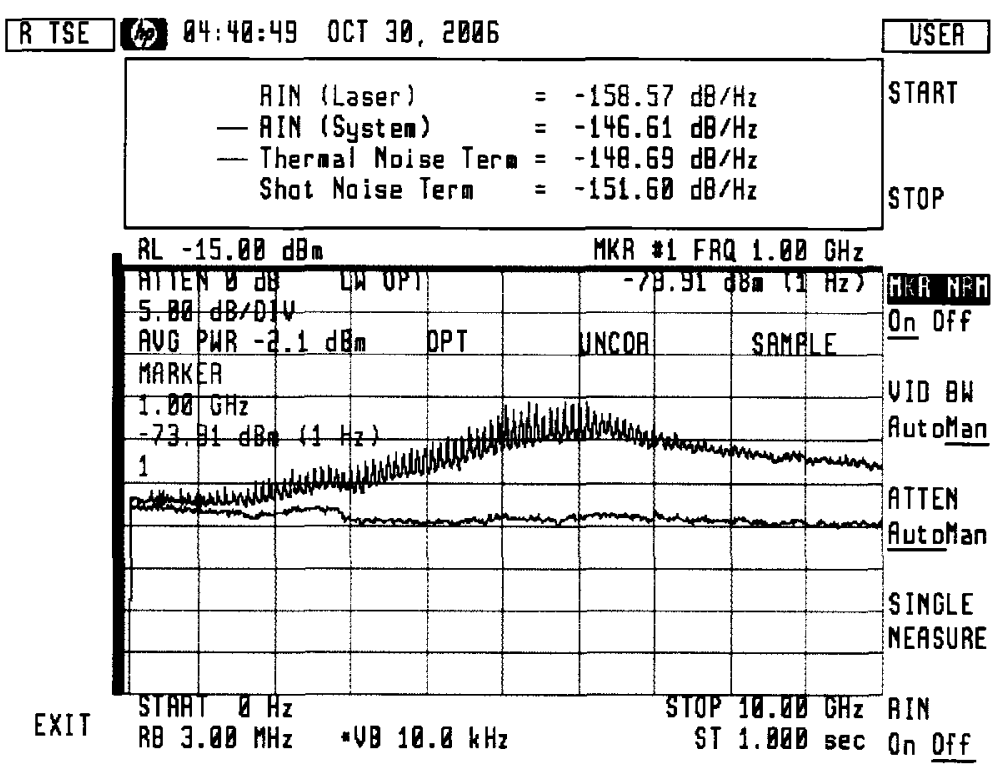

Figure 4.11: Measured relative intensity noise of $1300 \mathrm{~nm}$ DFB laser biased at $25 \mathrm{~mA}$ and measured at $1 \mathrm{GHz}$ frequency offset. 


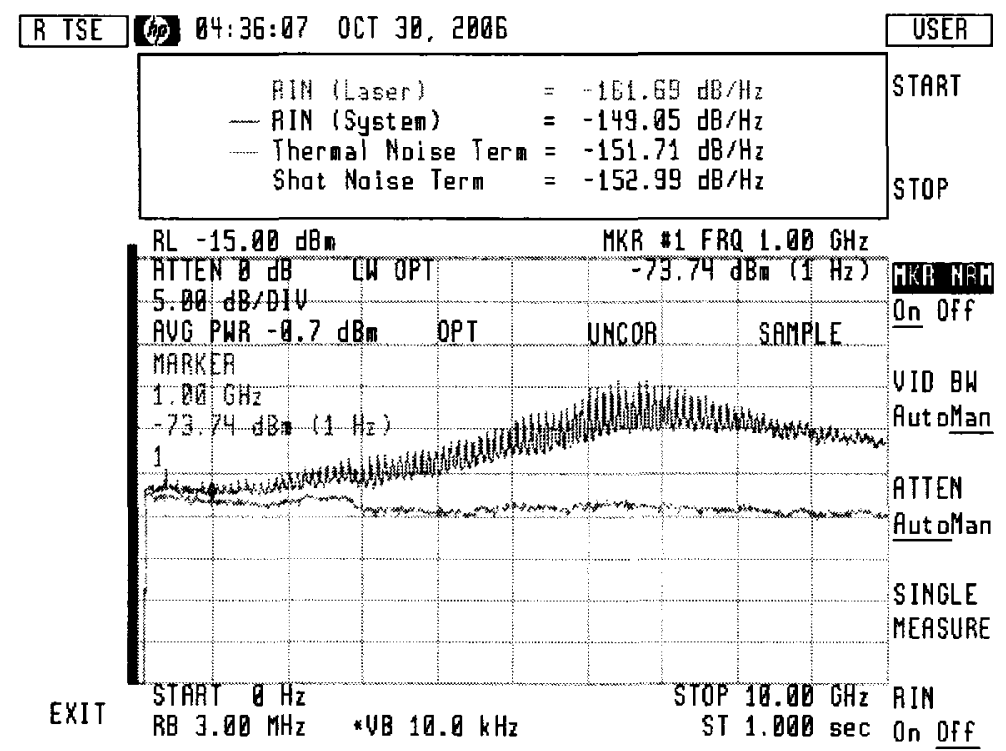

Figure 4.12: Measured relative intensity noise of $1300 \mathrm{~nm}$ DFB laser biased at $30 \mathrm{~mA}$ and measured at $1 \mathrm{GHz}$ frequency offset.

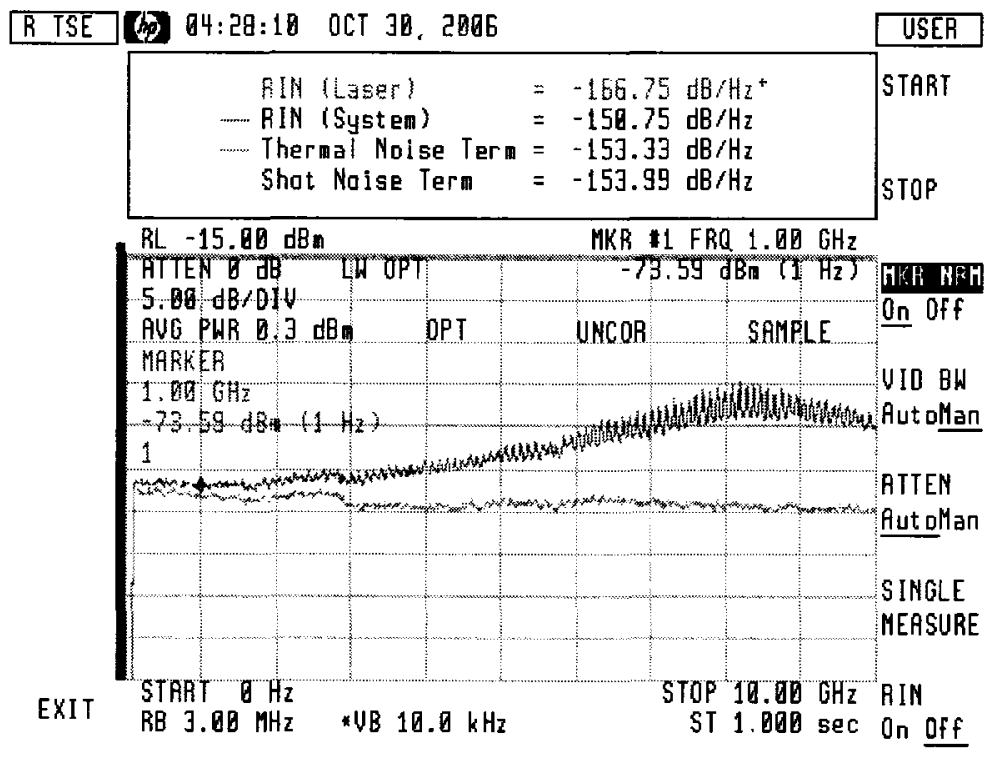

Figure 4.13: Measured relative intensity noise of $1300 \mathrm{~nm}$ DFB laser biased at $35 \mathrm{~mA}$ and measured at $1 \mathrm{GHz}$ frequency offset. 


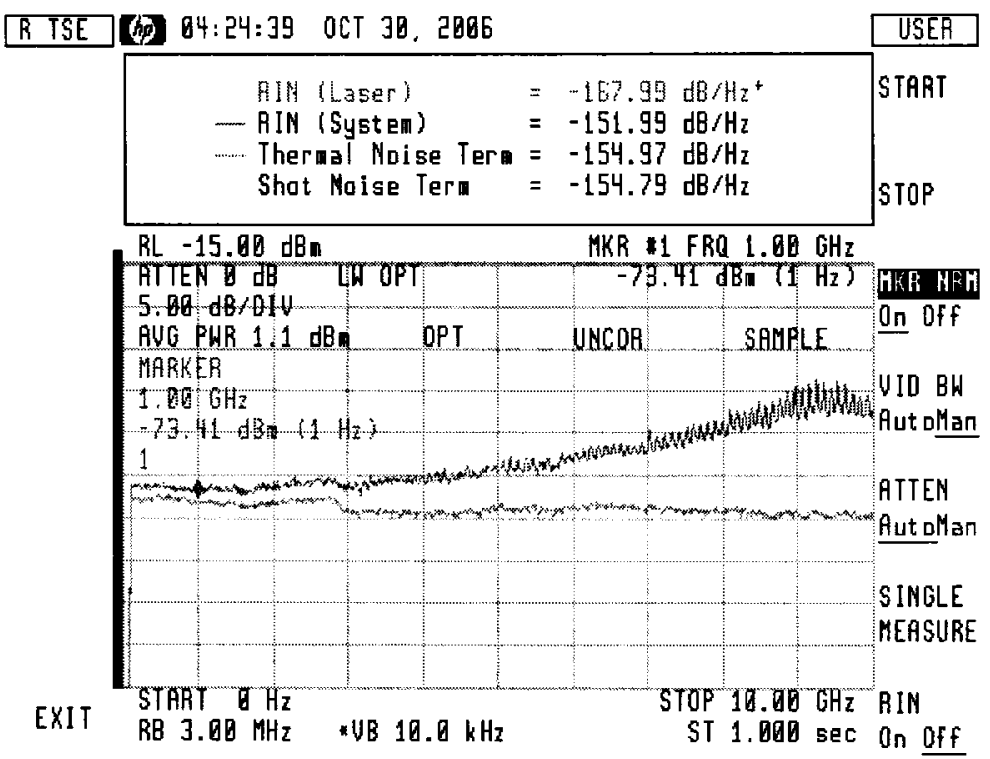

Figure 4.14: Measured relative intensity noise of $1300 \mathrm{~nm}$ DFB laser biased at $40 \mathrm{~mA}$ and measured at $1 \mathrm{GHz}$ frequency offset.

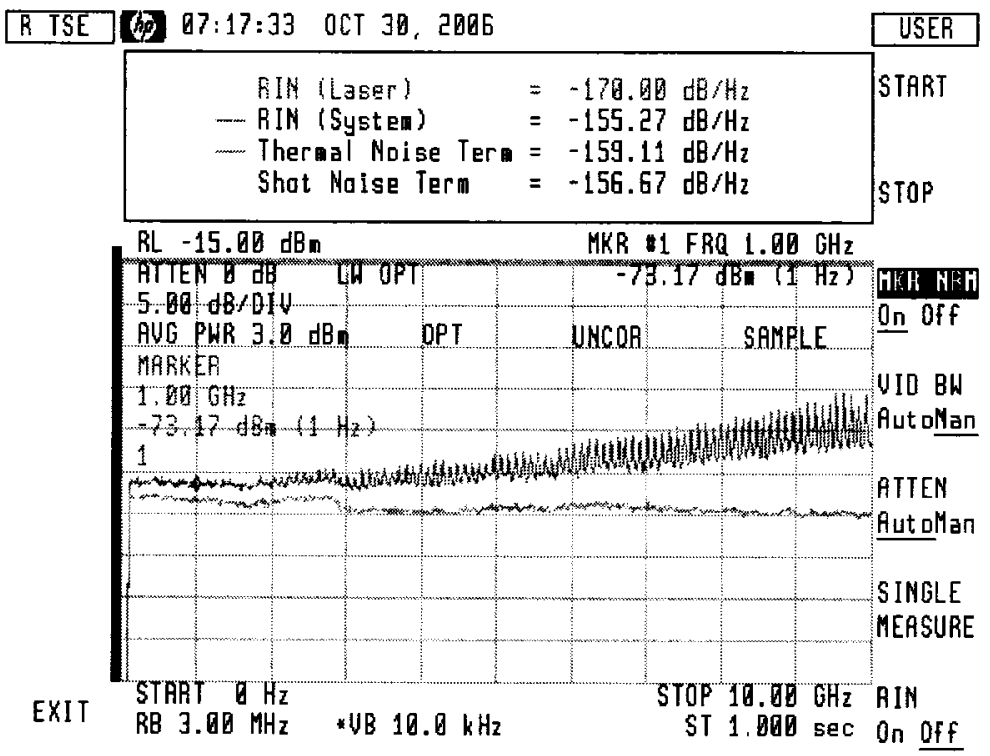

Figure 4.15: Measured relative intensity noise of $1300 \mathrm{~nm}$ DFB laser biased at $60 \mathrm{~mA}$ and measured at $1 \mathrm{GHz}$ frequency offset. 
towards higher values as the bias current increases. The above measured RIN for different bias levels are summarized in Table 4.1 and are plotted in Figure 4.16 on the next page.

Table 4.1: Measured average RIN at $1 \mathrm{GHz}$ frequency offset for different bias levels

\begin{tabular}{|c|c|}
\hline Bias Level $[\mathrm{mA}]$ & Measured average RIN $[\mathrm{dB} / \mathrm{Hz}]$ \\
\hline \hline 25 & -158.57 \\
\hline 30 & -161.69 \\
\hline 35 & -166.75 \\
\hline 40 & -167.99 \\
\hline 60 & -170 \\
\hline
\end{tabular}

Figure 4.16 on the following page shows the measured and simulated RIN as a function of the laser bias current for a fixed unintentional feedback level of $-45 \mathrm{~dB} / \mathrm{Hz}$. The results show that the RIN is a decreasing function of bias current; increasing bias current from $25 \mathrm{~mA}$ to $60 \mathrm{~mA}$ decreases the measured RIN value from $-158.57 \mathrm{~dB} / \mathrm{Hz}$ to $-170 \mathrm{~dB} / \mathrm{Hz}$. Agilent $71400 \mathrm{C}$ light wave signal analyzer can measure the laser RIN with an accuracy variations of $(3-6 \mathrm{~dB})$. The results demonstrate that the simulated and measured results are in a good agreement, the absolute error values for all measured points lie within a maximum allowable error level of $2 \mathrm{~dB}$ of the corresponding simulated values. The results also agree with findings reported in the literature [12].

\section{RIN versus external reflectivity}

Figures 4.17 on the next page -4.21 on page 79 show the measured average relative intensity noise of a $1310 \mathrm{~nm}$ DFB laser diode for bias current level of $15 \mathrm{~mA}$ and average external reflectivity ranging from $-45 \mathrm{~dB}$ to $-10 \mathrm{~dB}$. The rest of the measurement results are found in Appendix A.

The RIN spectrum of the DFB laser diode without intentional feedback is shown in Figure 4.17 on the next page. The RIN peak at around $3.5 \mathrm{GHz}$, which is the laser relaxation oscillation frequency at bias level of $15 \mathrm{~mA}$. The measured average RIN is $121.99 \mathrm{~dB} / \mathrm{Hz}$. When weak feedback, $-40 \mathrm{~dB}$, is applied to the DFB laser, the measured average RIN is slightly increased to $-120.43 \mathrm{~dB} / \mathrm{Hz}$ as shown in Figure 4.18 on page 78 . 


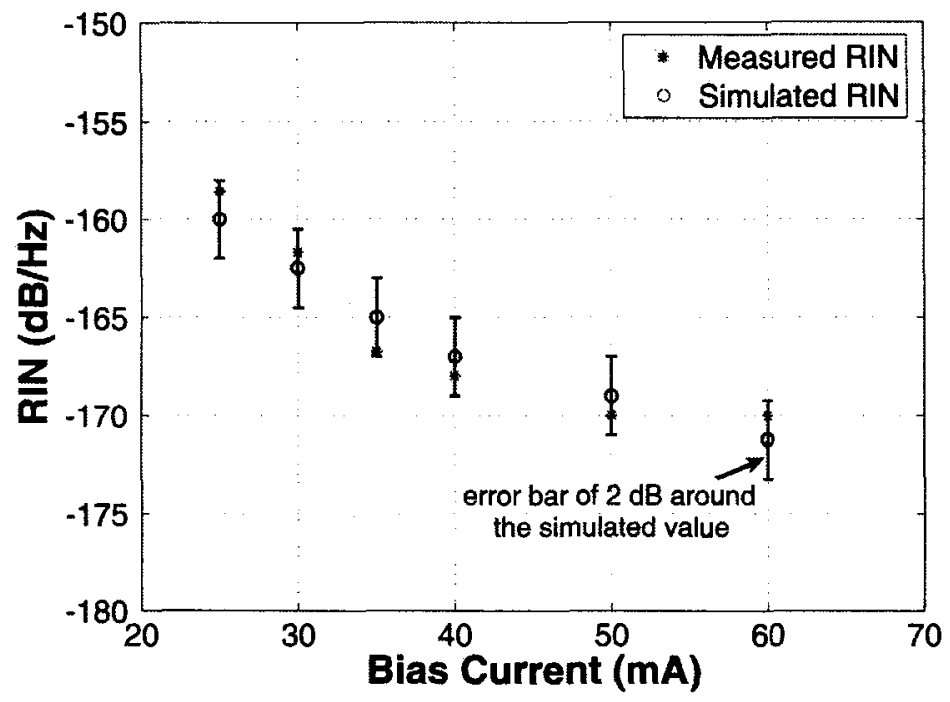

Figure 4.16: Measured and simulated relative intensity noise vs. bias current.

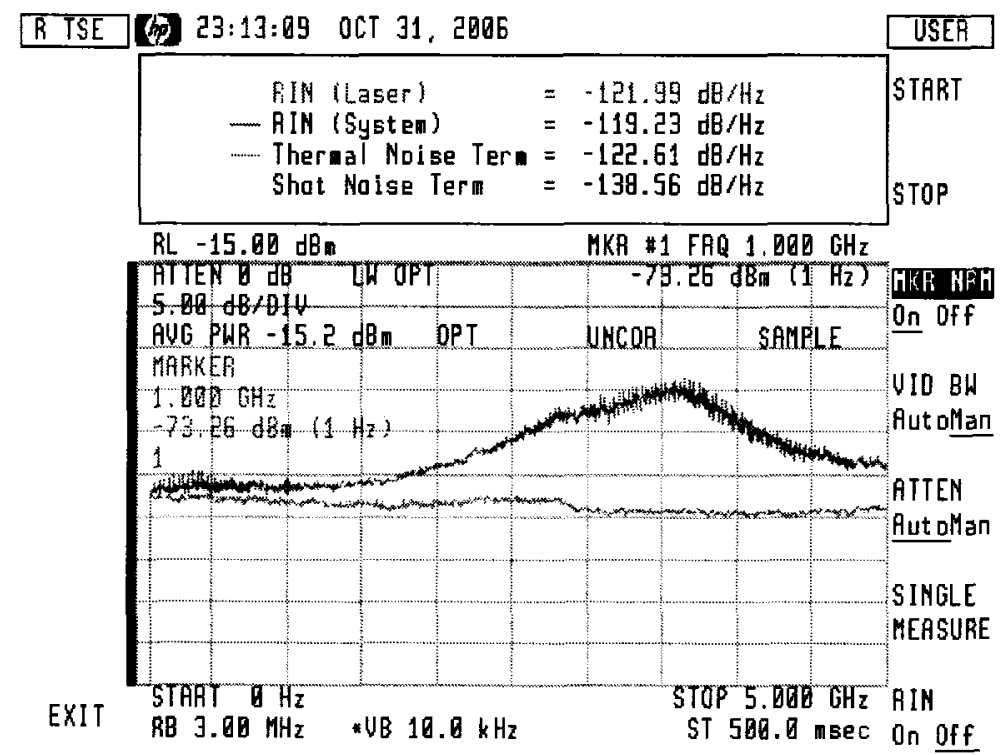

Figure 4.17: Measured relative intensity noise for bias current level of $15 \mathrm{~mA}$ and external reflectivity of $-45 \mathrm{~dB}$. 


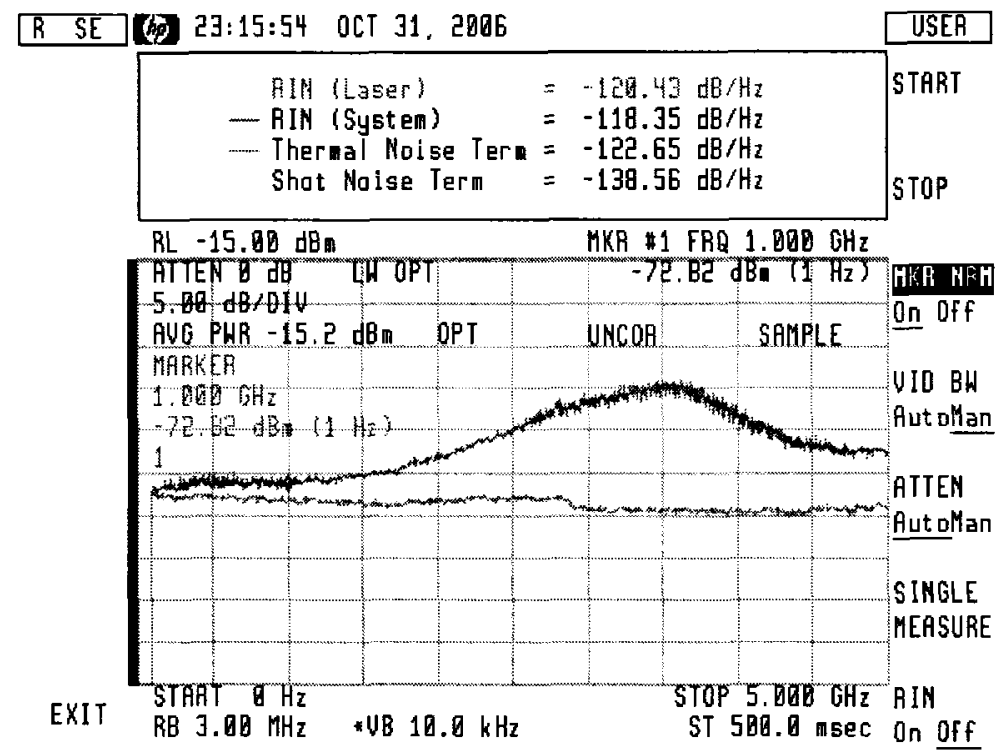

Figure 4.18: Measured relative intensity noise for bias current level of $15 \mathrm{~mA}$ and external reflectivity of $-40 \mathrm{~dB}$.

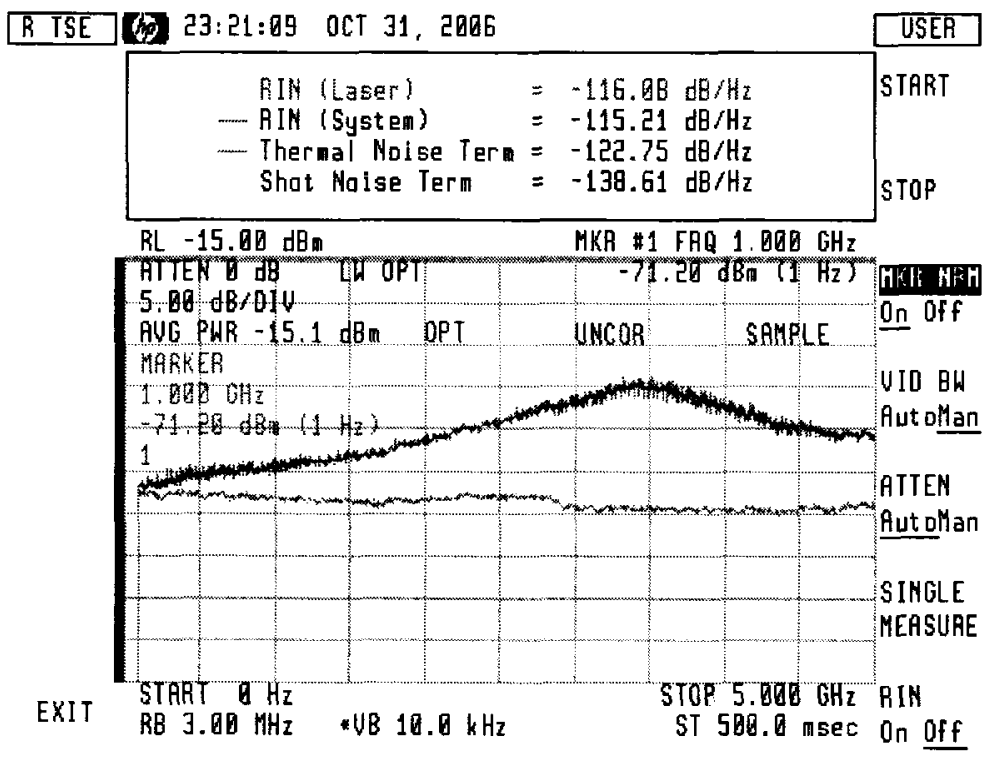

Figure 4.19: Measured relative intensity noise for bias current level of $15 \mathrm{~mA}$ and external reflectivity of $-27 \mathrm{~dB}$. 


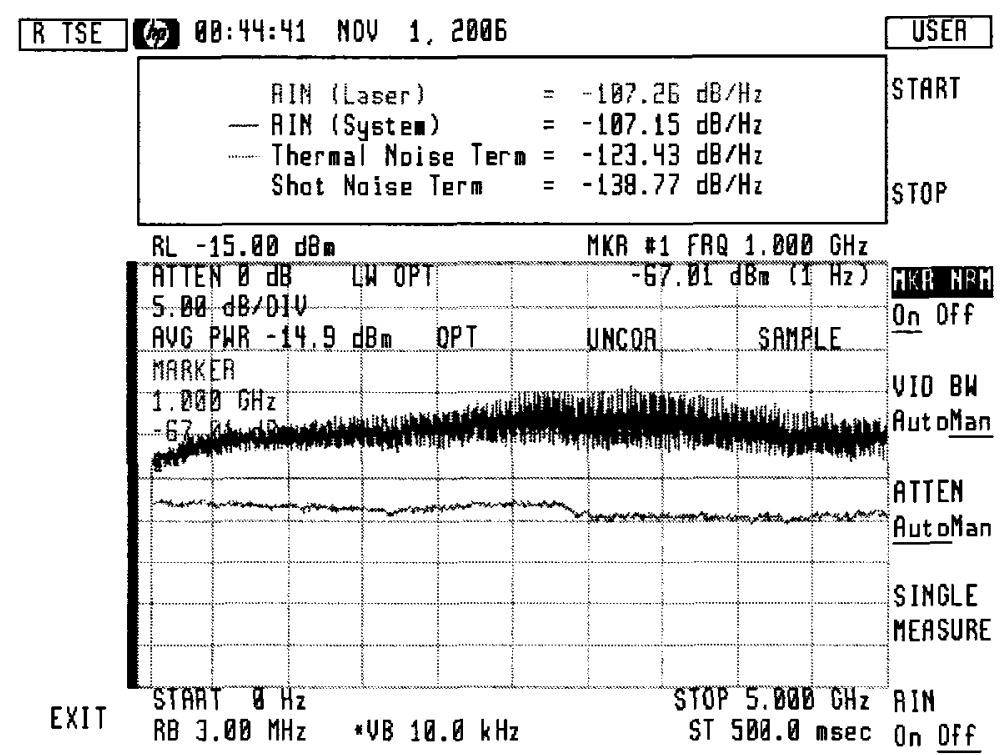

Figure 4.20: The measured relative intensity noise for bias current level of $15 \mathrm{~mA}$ and external reflectivity of $-20 \mathrm{~dB}$.

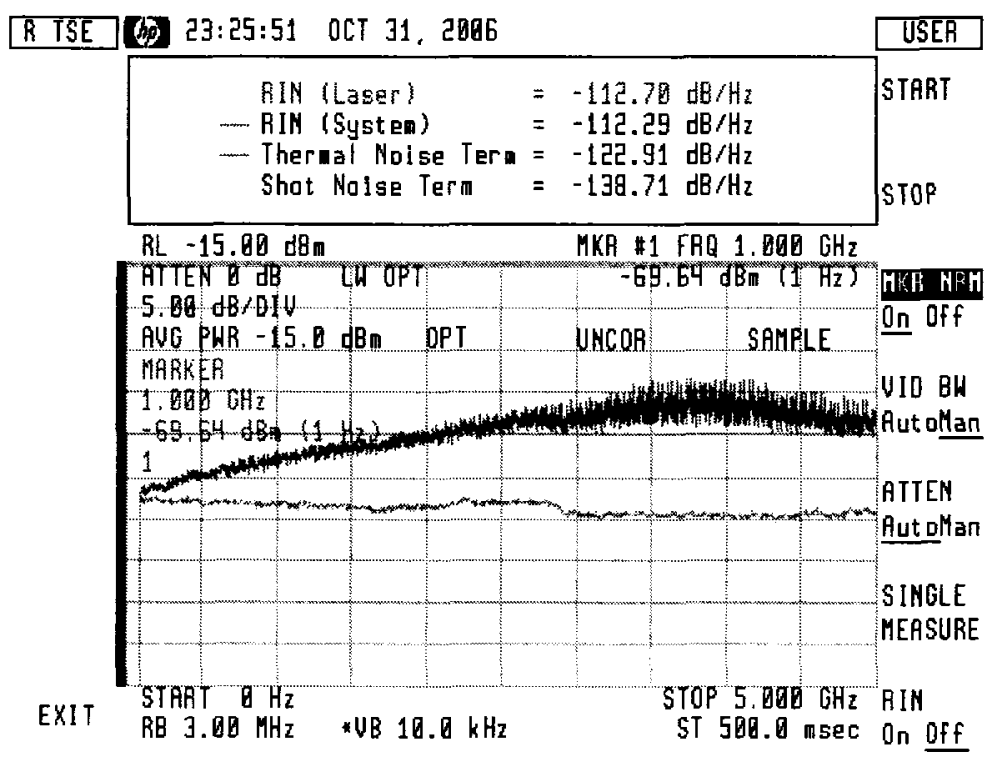

Figure 4.21: Measured relative intensity noise for bias current level of $15 \mathrm{~mA}$ and external reflectivity of $-10 \mathrm{~dB}$. 
A bigger increase in the RIN is observed at higher feedback as shown in Figure 4.19 on page 78. The measured average RIN increased to $-116.08 \mathrm{~dB} / \mathrm{Hz}$ when a feedback level of $-27 \mathrm{~dB}$ was applied to the DFB laser. A considerable increase in the RIN spectrum is observed when the feedback is further increased to $20 \mathrm{~dB}$ due to the operation in the coherence collapse regime. The measured average RIN in this case was $-107.26 \mathrm{~dB} / \mathrm{Hz}$ as shown in Figure 4.20 on the preceding page. At this regime the laser emits in random phase, the linewidth of the emitted light is much broadened, and no distinct peak for the noise spectrum can be observed. Increasing the feedback to very high level, $10 \mathrm{~dB}$, caused the laser to reach the stable feedback Regime V. The measured average RIN was $-112.70 \mathrm{~dB} / \mathrm{Hz}$ as shown in Figure 4.21 on the previous page. More reduction in the RIN of the DFB laser is expected to occur for higher feedback. The available feedback in our experiments is limited to only $10 \mathrm{~dB}$ due to the insertion loss of the various used equipment.

The above measured average RIN results for different feedback levels are summarized in Table 4.2 and are plotted in Figure 4.22 on the following page. The figure shows that the average RIN increases slightly as the external reflectivity increases. However, when the feedback level exceeds a certain level ( $-30 d B$ in these measurements), the measured relative intensity noise of the laser exhibits higher values due to the laser operation in Regime IV, the coherence collapse regime. A further increase in the feedback level (greater than $-15 d B$ in these measurements ) forced the DFB laser diode into feedback Regime $\mathrm{V}$ with low measured relative intensity noise. The simulation results were included in Figure 4.22 to facilitate a comparison with the measured results. Agilent $71400 \mathrm{C}$ lightwave signal analyzer can measure the laser RIN with an accuracy variations of $(3-6 \mathrm{~dB})$. The simulated and measured results are in good agreement for feedback regimes I, II, III, and IV, the absolute error values for all measured points lie within a maximum allowable error level of $5 \mathrm{~dB}$ of the corresponding simulated values. These results are also in good agreement with results presented in [30] and [42].

Although the laser rate equations that include feedback and noise terms were normalized to improve the simulator numerical solvers convergence, the model was unable to simulate the RIN in feedback Regime $\mathrm{V}$ and faced convergence difficulties. It was observed that the ADS numerical solver was unable to solve these rate equations as the laser enters feedback Regime $\mathrm{V}$, this is attributed to the high reflection level in Regime V. 
Table 4.2: Measured average RIN at $1 \mathrm{GHz}$ frequency offset for $15 \mathrm{~mA}$ bias current and different feedback levels

\begin{tabular}{|c|c|}
\hline Feedback Level $[\mathrm{dB}]$ & Measured average RIN $[\mathrm{dB} / \mathrm{Hz}]$ \\
\hline \hline-45 & -121.99 \\
\hline-42 & -120.88 \\
\hline-40 & -120.43 \\
\hline-36 & -120.51 \\
\hline-33 & -120.08 \\
\hline-29 & -119.80 \\
\hline-27 & -116.08 \\
\hline-24 & -112.03 \\
\hline-20 & -107.26 \\
\hline-15 & -109.06 \\
\hline-10 & -112.70 \\
\hline
\end{tabular}

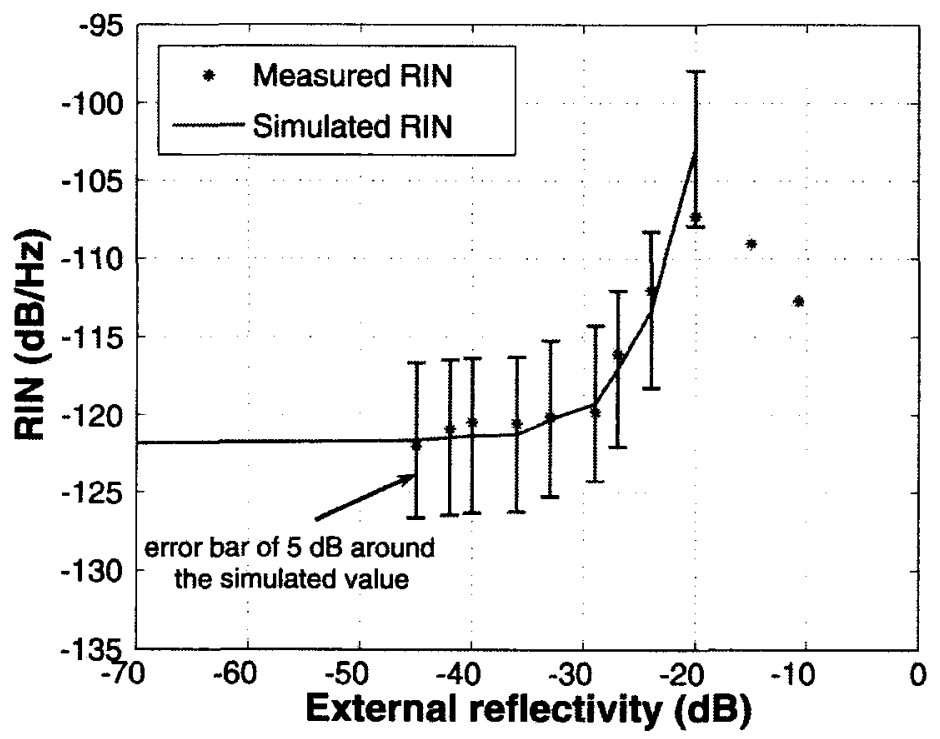

Figure 4.22: Measured and simulated RIN $[d B / H z]$ of DFB for different external reflectivities $[d B], I_{b i a s}=12 \mathrm{~mA}, \lambda=1310 \mathrm{~nm}$. 
It is worth noting that the drop that exists at a frequency $2.9 \mathrm{GHz}$ is due to the fact that the Agilent $71400 \mathrm{C}$ lightwave signal analyzer uses two different tracking generator modules to cover the full frequency range from $20 \mathrm{~Hz}$ to $18 \mathrm{GHz}$. The first module covers frequencies ranging from $20 \mathrm{~Hz}$ to $2.9 \mathrm{GHz}$, while the second covers frequencies ranging from $2.7 \mathrm{GHz}$ to $18 \mathrm{GHz}$. The drop that exists at a frequency of $2.9 \mathrm{GHz}$ is due to the transition from the first module to the second.

\subsubsection{Modulation Frequency Response Measurements}

The modulation frequency response of the DFB laser diode at different optical backreflections levels was measured using the experimental setup shown in Figure 4.23 on the next page. The DFB laser diode was mounted on the THORLABS TCLDM9 $5.6 \mathrm{~mm} / 9 \mathrm{~mm}$ laser diode mount. It was temperature controlled and biased by the ILX lightwave LDC$3724 B$ laser diode controller. The emission wavelength for the DFB laser diode was $1310 \mathrm{~nm}$. The DFB laser diode output is sent to the $\mathrm{T}$ port of the FOBS, where it was divided. Half of the power was coupled to the Agilent $71400 \mathrm{C}$ lightwave signal analyzer to measure the modulation frequency response. The other half of the power was directed to the OZ Optics fiber optic reflector to achieve the desired back reflection level. The feedback level was controlled by the OZ Optics variable attenuator. The OZ Optics Polarization controller was used to guarantee that the reflected power back into the laser had the same polarization as the emitted power. The reflected power through the $\mathrm{R}$ port of the FOBS was measured using the ILX Lightwave OMM-6810B Optical power and wavelength meter.

In this experiment, an Agilent RF tracking generator (70300A, $100 \mathrm{~Hz}$ to $2.9 \mathrm{GHz}$ ) and an Agilent micro-wave tracking generator (70301A, $2.7 \mathrm{GHz}$ to $18 \mathrm{GHz}$ ) from the Agilent $71400 \mathrm{C}$ lightwave signal analyzer were used to provide a sweep modulation frequency source whose frequency was synchronized with the sweep of the spectrum analyzer. The RF output of the $70300 \mathrm{~A}$ was connected to the RF input of $70301 \mathrm{~A}$ to cover the full range of frequency (100 HZ to $18 \mathrm{GHZ}$ ). The output of the tracking generator was used to directly modulate the laser diode using an RF cable connected to a standard RF-SMA connector in the laser mount. The mount is designed with a bias- $T$ that accepts a $50 \Omega$ impedance modulation input through a standard RF-SMA connector. The modulated light enters the 
lightwave section in the $71400 \mathrm{C}$ system through a Single-Mode Fiber (SMF) connected to the front-panel input connector. The optical power is detected, collimated, amplified, and then focused onto a $22 \mathrm{GHz}$ bandwidth InGaAs photodetector using the Agilent 70810B lightwave section. The data was displayed on a 70004A display unit and acquired through a GPIB (IEEE488.2 interface) to a personal computer for obtaining screen images and collecting trace data using Agilent N1031A BenchLink lightwave software.

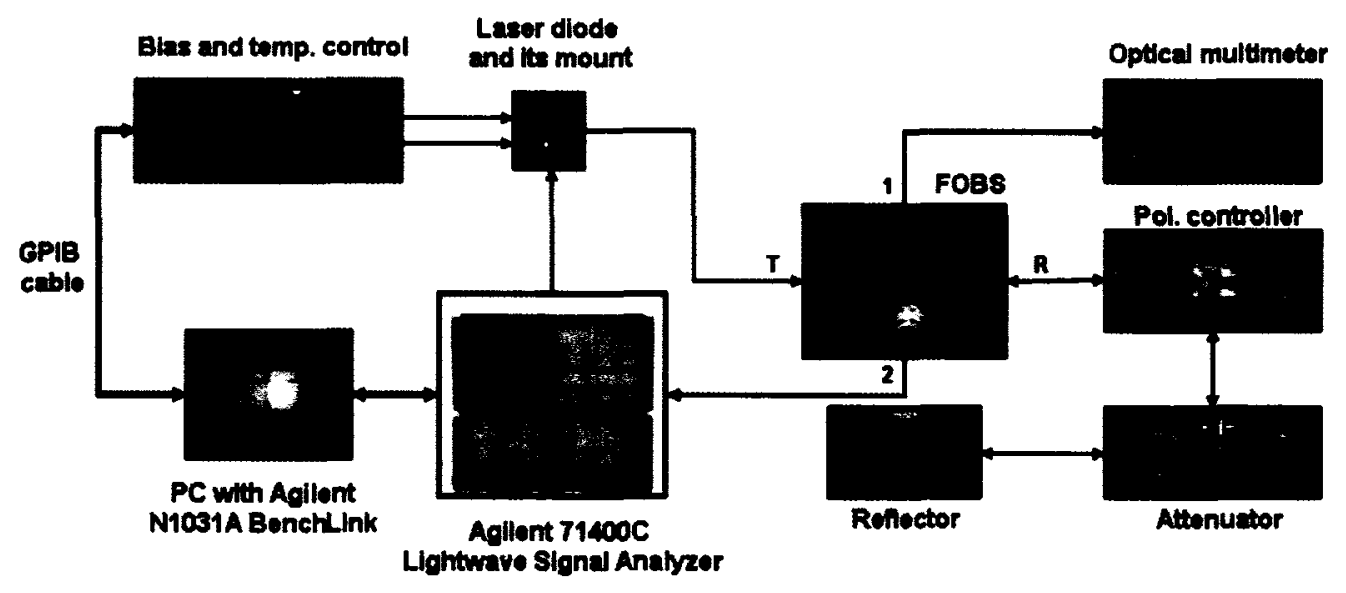

Figure 4.23: Modulation frequency response measurement experimental setup.

Figure 4.24 on the following page through Figure 4.27 on page 85 show the measured modulation frequency response of $1300 \mathrm{~nm}$ DFB laser diode biased at $30 \mathrm{~mA}$, with different feedback levels.

The measured relaxation oscillation frequency was $6.444 \mathrm{GHz}$ with optical back-reflections of $-45 \mathrm{~dB}$, and $6.472 \mathrm{GHz}$ with optical back-reflections of $-30 \mathrm{~dB}$ as shown in Figures 4.24 and 4.25 respectively. When the feedback level was increased from $-15 \mathrm{~dB}$ to $-10 \mathrm{~dB}$ the measured relaxation oscillation frequency increased from $6.506 \mathrm{GHz}$ to $6.534 \mathrm{GHz}$ as shown in Figures 4.26 on page 85 and 4.27 on page 85 respectively.

\subsubsection{Distortion Measurements Setup and Results}

The linearity performance of the laser is determined by measuring different types of distortion that are caused by the laser. In this experiment, Agilent $71400 \mathrm{C}$ lightwave signal 


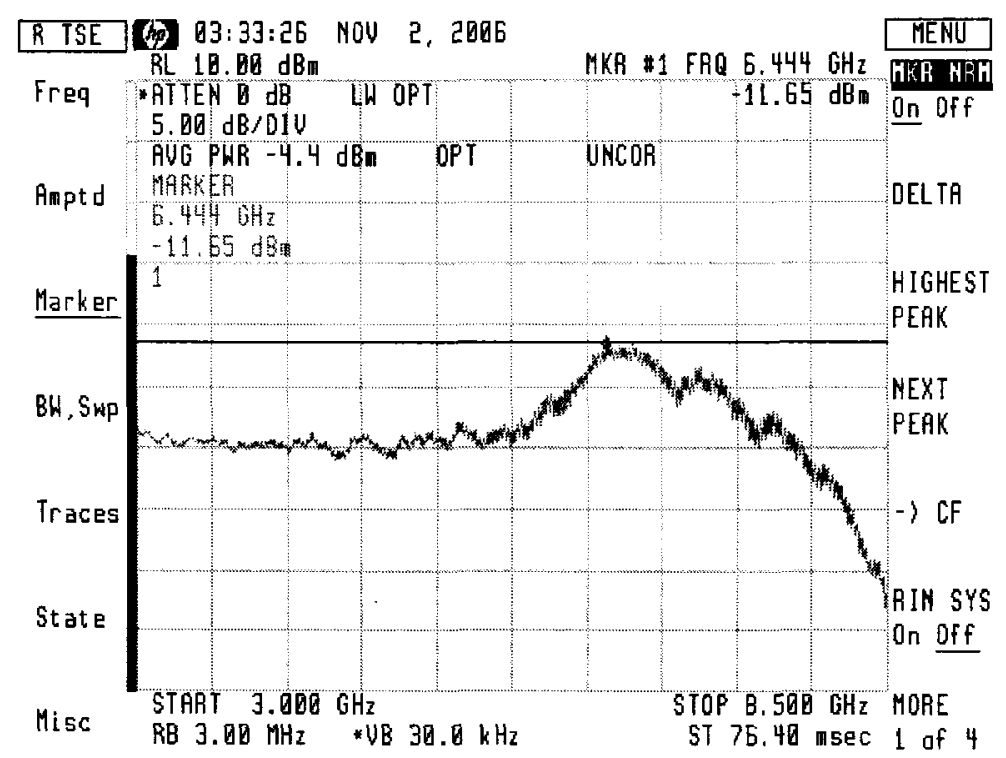

Figure 4.24: The measured relaxation oscillation frequency of the $1310 \mathrm{~nm}$ DFB laser diode biased at $30 \mathrm{~mA}$ with $-45 \mathrm{~dB}$.

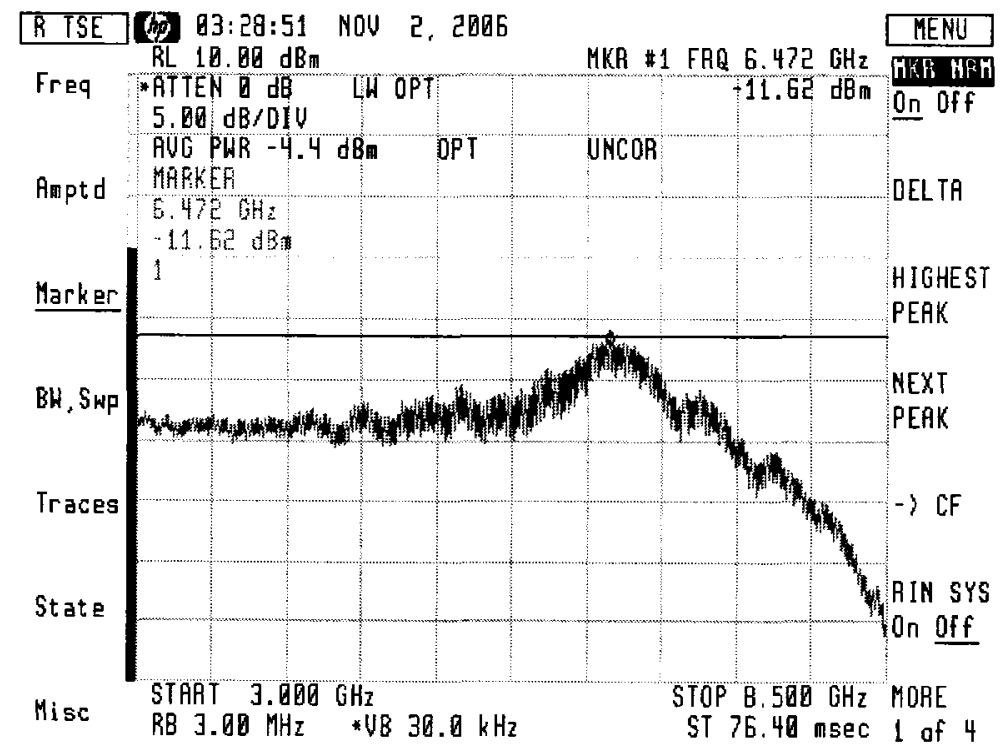

Figure 4.25: Measured relaxation oscillation frequency of the $1310 \mathrm{~nm}$ DFB laser diode biased at $30 \mathrm{~mA}$ with $-30 \mathrm{~dB}$. 


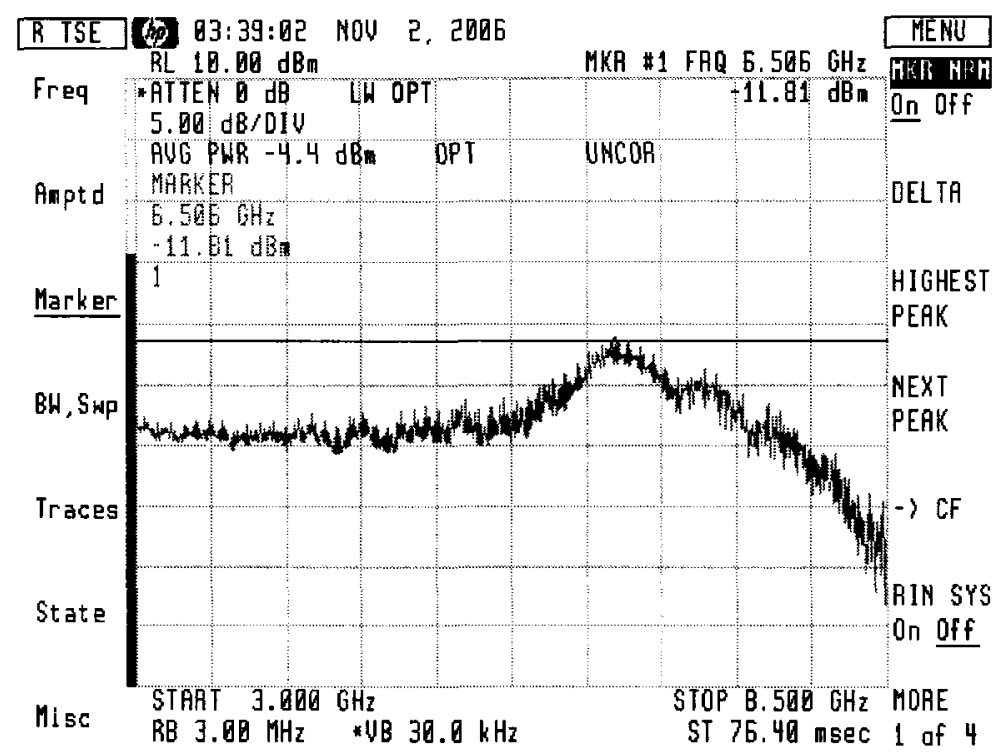

Figure 4.26: Measured relaxation oscillation frequency of the $1310 \mathrm{~nm}$ DFB laser diode biased at $30 \mathrm{~mA}$ with $-15 \mathrm{~dB}$.

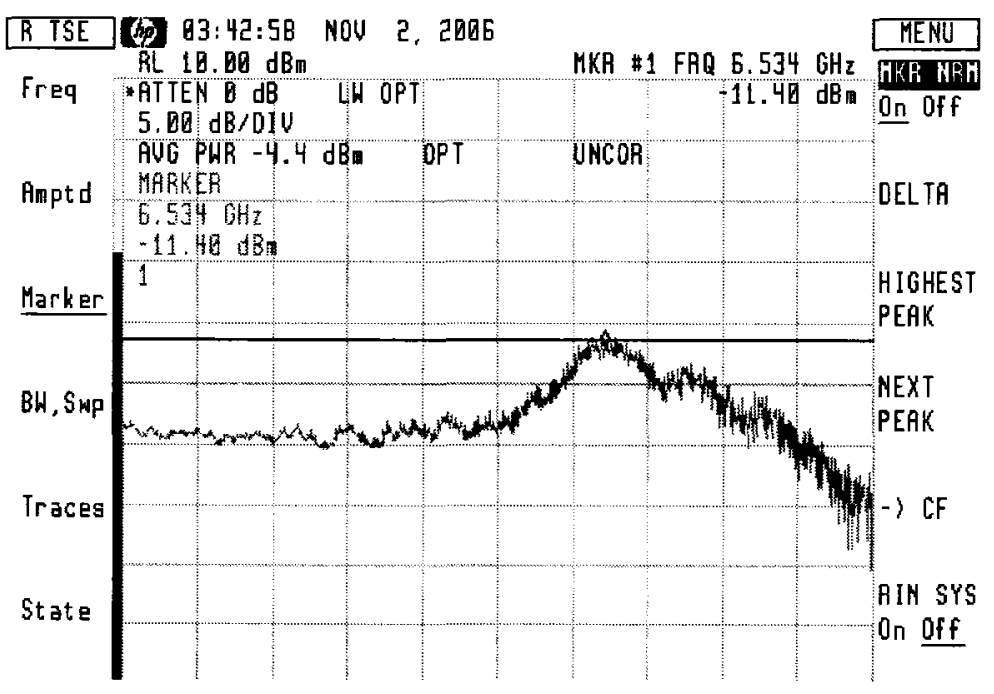

Figure 4.27: TMeasured relaxation oscillation frequency of the $1310 \mathrm{~nm}$ DFB laser diode biased at $30 \mathrm{~mA}$ with $-10 \mathrm{~dB}$. 
analyzer and Agilent E4438C ESG vector signal generator were used to measure the second and third order harmonic distortion in presence of feedback. The setup was similar to that of modulation frequency response measurement that was presented in the previous section, except that the output from E4438C ESG signal generator was used to modulate the DFB laser diode through the $\mathrm{T}$ bias of the laser mount. Figure 4.28 shows the detailed block diagram of the experimental setup for the distortion measurement in presence of optical back-reflections.

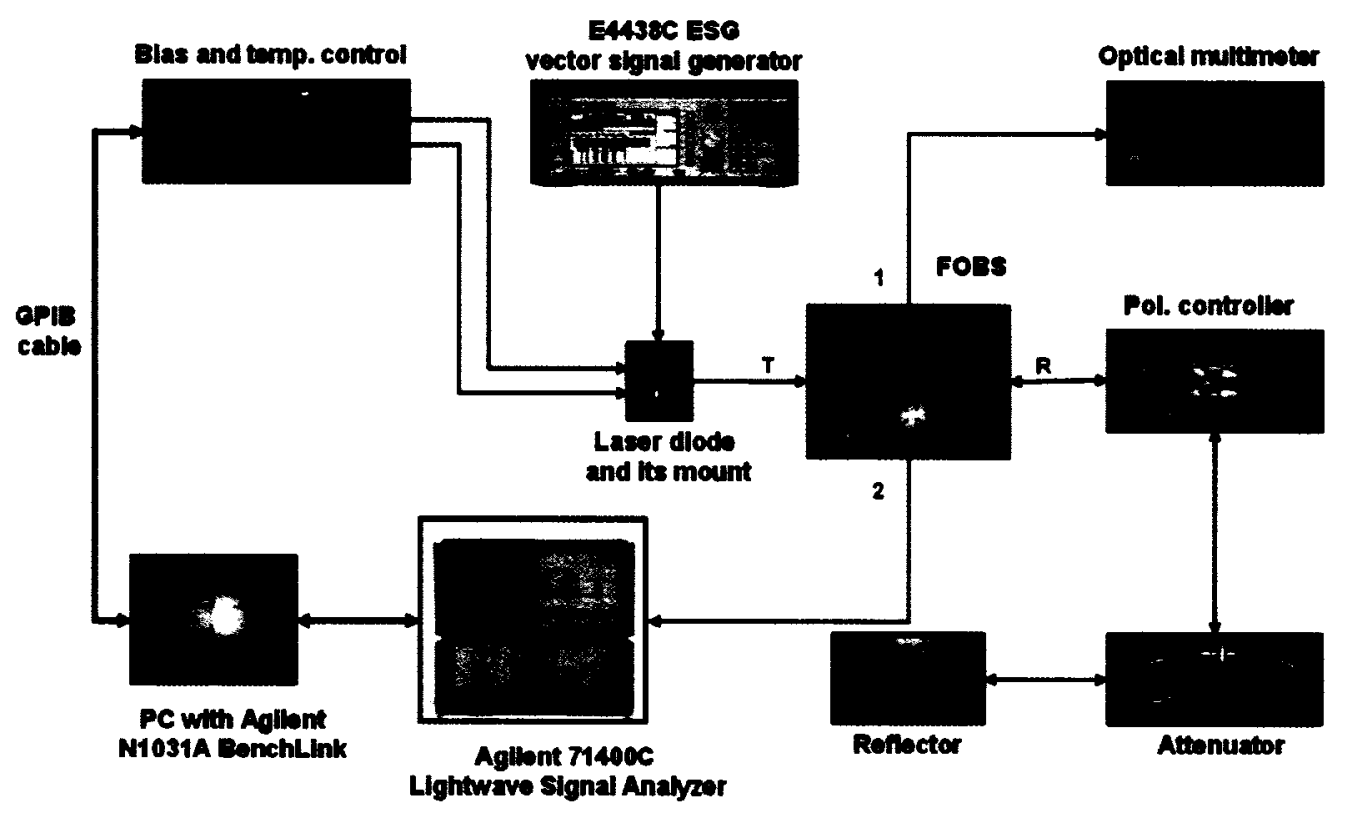

Figure 4.28: Distortion measurement experimental setup.

Figure 4.29 on the next page shows the measured second order harmonic distortion of DFB laser at different feedback levels normalized to the fundamental tone power. The results show that as the feedback level increases, the second order harmonic distortion increases. At a bias current of $20 \mathrm{~mA}$, the measured second order harmonic distortion was $-17 \mathrm{~dB}$ for the isolated DFB laser, $-15 \mathrm{~dB}$ for the same DFB laser with $-45 \mathrm{~dB}$ backreflection, and $-12 \mathrm{~dB}$ for the same DFB laser with $-15 \mathrm{~dB}$ back-reflection. The results also show that the second order harmonic distortion is a decreasing function of the bias current for all of feedback levels. 


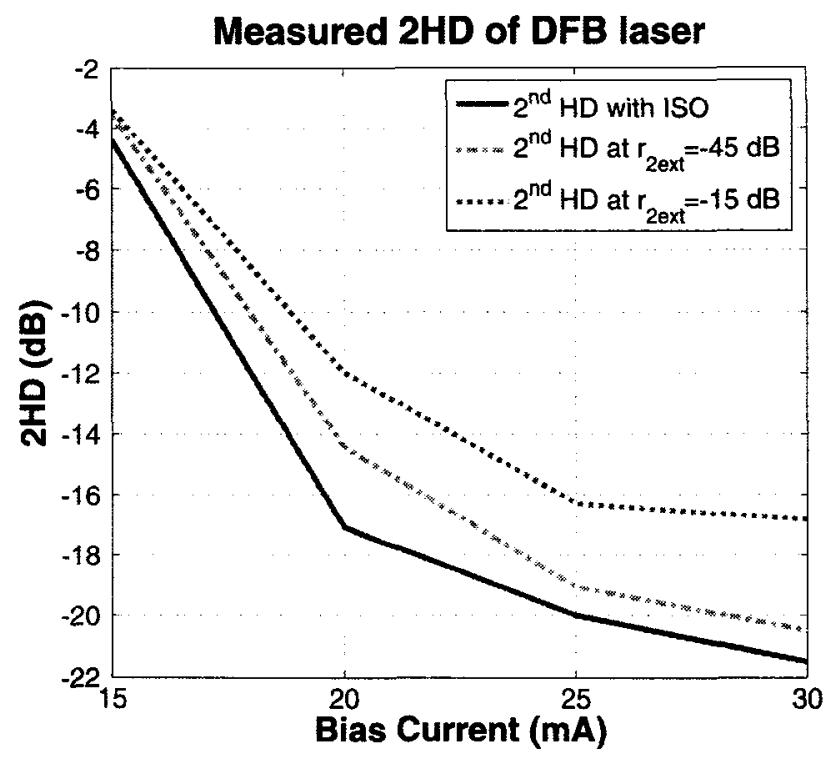

Figure 4.29: Measured second order harmonic distortion of DFB laser at different feedback levels.

Figure 4.30 on the following page shows the measured third order harmonic distortion of the DFB laser at different feedback levels normalized to the fundamental tone power. The results show that as the feedback level increases, the third order harmonic distortion increases. At a bias current of $20 \mathrm{~mA}$, the measured third order harmonic distortion is $-18 \mathrm{~dB}$ for the isolated DFB laser, $-17 \mathrm{~dB}$ for the same DFB laser with $-45 \mathrm{~dB}$ backreflection, and $-13 \mathrm{~dB}$ for the same DFB laser with $-15 \mathrm{~dB}$ back-reflection. The results also show that the third order harmonic distortion is a decreasing function of the bias current for of all feedback levels. 


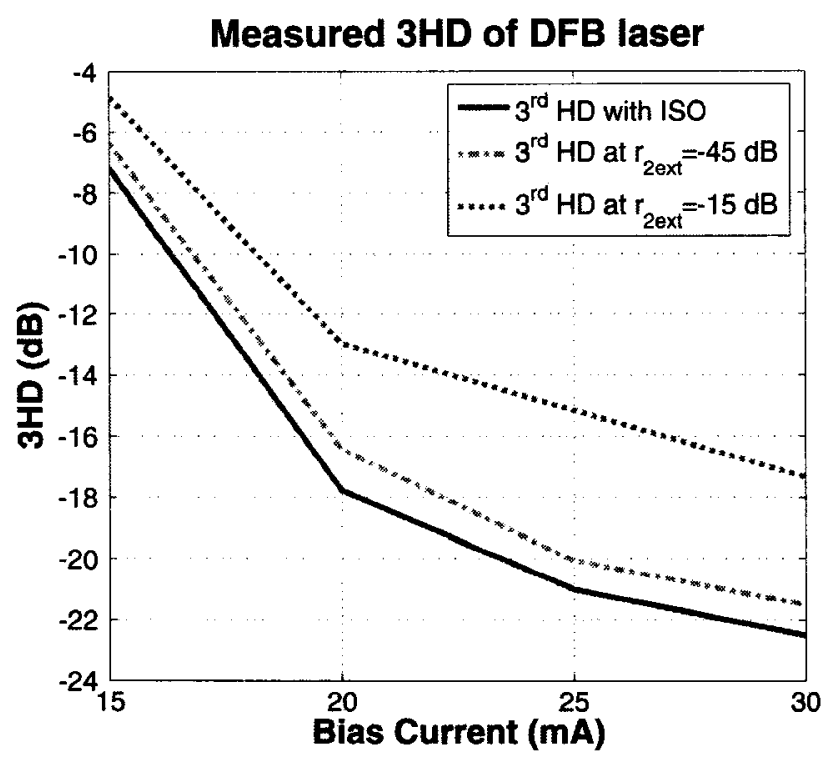

Figure 4.30: Measured third order harmonic distortion of DFB laser at different feedback levels.

\subsection{Summary}

This chapter contains various experimental setups that have been used to examine the laser performance with optical feedback as well as to verify the simulation results. Different DFB laser spectra were shown for different optical feedback levels. An induced tunable backreflections setup were presented to measure laser's DC characteristics, noise, and linearity using the Agilent $71400 \mathrm{C}$ lightwave signal analyzer. The simulation and measurement results were compared and the results closely matched. 


\section{Chapter 5}

\section{FBG Design and Implementation}

\subsection{Introduction}

In this chapter the design and implementation of the Fiber Bragg Grating (FBG) will be presented. The required FBG parameters will be determined based on the laser output characteristics, and the required back-reflection level needed to drive the laser to the feedback Regime V. Simple analytical formulas were used to determine the primarily design parameters, then the OptiGrating design software was used to design the desired FBG. Finally the FBG was implemented in the laboratory.

\subsection{FBG Introduction and Basic Calculations}

\subsubsection{FBG Principles}

The FBG consists of a fiber segment whose core refraction index varies periodically along its length as shown in Figure 5.1 on the next page. This refractive index variation is formed by exposure of the fiber core to an intense optical interference pattern [55]. The FBG has many advantages, such as: achieving all-fiber geometry, low insertion loss, and low cost. In addition to its flexibility, it is able to achieve the desired spectral characteristics [56]. The FBGs can be used in many applications including DFB lasers, all fiber lasers, optical filters for WDM systems, dispersion compensation in optical communications, and optical sensors [57]. In this work the FBG will be attached to the laser pigtail to work as a reflector. Although the presence of the FBG will reduce the laser output, the optical feedback from the FBG will reduce the laser threshold current which will compensate for 
the power reduction, and give almost the same P-I characteristics of a laser without the attached FBG [58].

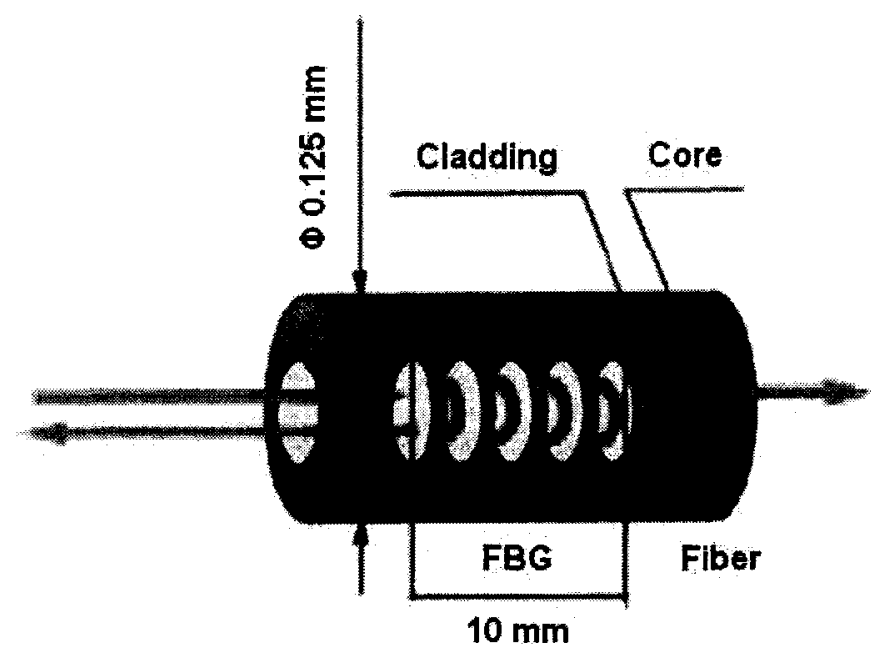

Figure 5.1: FBG structure

The coupled mode theory can be used to analyze the field propagation in the FBG, and to obtain the quantitative information about the diffraction efficiency and spectral characteristics of the FBG. It provides a straightforward technique that can model the optical properties of the FBG accurately [56]. The coupled mode approach involves the numerical solution of two coupled differential equations. An analytic solution is possible in case of uniform FBGs. Also, matrix methods such as the transfer matrix method can be used in the analysis; where the FBG is divided into sections, the length of each section is larger than the largest corrugation period. The variation of the refraction index of each section is considered as a uniform grating described by a transfer matrix, the product of the individual matrices give the global matrix which characterizes the FBG [57].

The FBG is characterized by its length and grating period, which can be obtained from the following relations:

$$
\begin{aligned}
& 2 n_{\text {eff }} \Lambda=\lambda_{\text {Bragg }} \\
& r_{\text {max }}=\tanh ^{2}(\kappa L)
\end{aligned}
$$


where $n_{\text {eff }}$ is the effective refractive index, $\Lambda$ is the grating period, $\lambda_{B r a g g}$ is the grating

wavelength, $r_{\max }$ is the Bragg grating maximum reflectivity, $r_{\max }$ is the Bragg grating maximum reflectivity, $\mathrm{L}$ is the grating length, $\kappa$ is the grating coupling coefficient.

\subsubsection{FBG Parameter Calculations}

\section{Laser output parameters}

The output of the Laser mate $1550 \mathrm{~nm}$ MQW-DFB pigtailed laser diode, mounted on the the THORLABS TCLDM9 $5.6 \mathrm{~mm} / 9 \mathrm{~mm}$ mount and driven by the ILX lightwave LDC3724B laser diode controller, was measured with an Agilent 86140B Optical Spectrum Analyzer to determine the operating wavelength at different bias currents. Figure 5.2 on the following page shows the DFB laser diode output at bias current of $12 \mathrm{~mA}, 15 \mathrm{~mA}, 25 \mathrm{~mA}$, $35 \mathrm{~mA}$, and $40 \mathrm{~mA}$. The results show that as the bias current increases the laser emission wavelength shifts towards higher values, the laser emission wavelength was concentrated around $1553 \mathrm{~nm}$ with a deviation of $\pm 0.5 \mathrm{~nm}$. With these results, the FBG wavelength have to match to this wavelength range.

\section{FBG parameters}

As shown in the previous section, the FBG wavelength should be designed for a wavelength of $1553 \pm 0.5 \mathrm{~nm}$. From the previous chapter, the feedback level required to cause the DFB laser diode to operate in feedback Regime $V$ should be greater than $-10 \mathrm{~dB}$. The FBG period and length can be calculated from ( 5.2 .1 on the previous page) and (5.2.2 on the preceding page), for a feedback level of $-7 \mathrm{~dB}$, the FBG period is $\Lambda=0.5257 \mu \mathrm{m}$ and the FBG coupling-length product $\kappa L$ is 0.4812 .

\subsection{OptiGrating Design Software}

OptiGrating 4.2.1 is a powerful modeling software that offers many different options for analyzing and designing FBGs. The FBG shape, length, apodization, and chirp can be adjusted according to the fiber diameter and refractive index. The OptiGrating $4.2 .1 \mathrm{can}$ perform the necessary numerical simulations based on solving the coupled mode equations using the transfer matrix method. The detailed FBG design steps using OptiGrating 4.2.1 


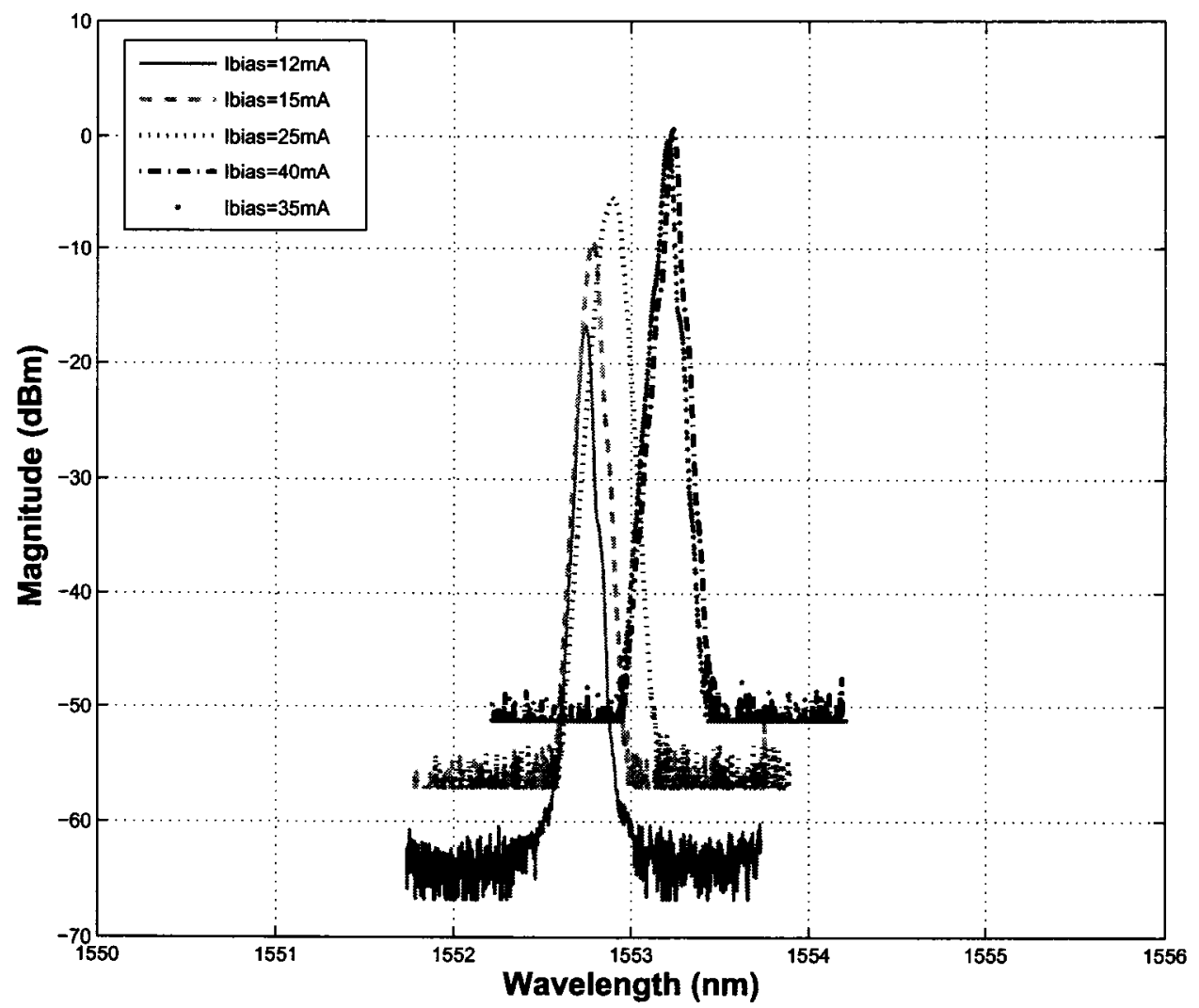

Figure 5.2: $1550 \mathrm{~nm}$ DFB laser diode spectrum at different bias current levels. 
are included in Appendix B.

\subsection{FBG Implementation}

\subsubsection{FBG Implementation Procedures}

The grating is fabricated through an intense ultraviolet (UV) light irradiation using a phase mask as explained below.

\section{Optical fiber preparation}

As the ultraviolet can not well penetrate the acrylic (or polyimide) coatings that are on a standard optical fiber, a small section of the buffer material should be removed $(2 \mathrm{~cm})$ to expose the silica cladding. The optical fiber is placed in the appropriate fiber holder and temporary connectors are used on the fiber ends to monitor the Bragg grating with the source and optical spectrum analyzer.

\section{Phase mask}

Phase masks are corrugated circular sheets of silica. Each phase mask has a different pitch (periodicity) to the corrugated ridges on its surface. Bragg grating wavelength is determined by the phase mask pitch. The phase mask functions to split the incoming UV light into multiple diffracted beams as shown in Figure 5.3.

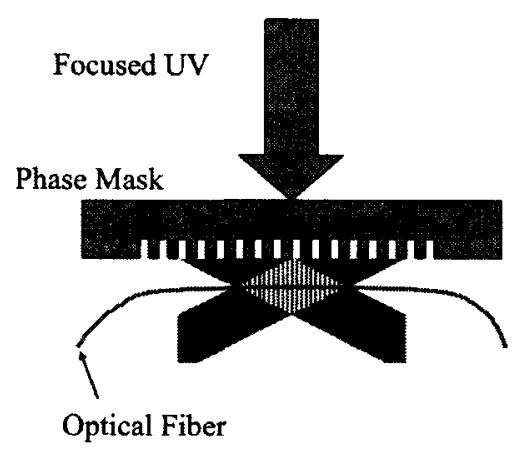

Figure 5.3: Phase mask. 


\section{Interferometric (Holographic) technique}

The interferometric technique creates the UV interference pattern by splitting the incoming UV into two separate light paths and recombining them at the optical fiber location as shown in Figure 5.4. The splitting is done using the phase mask; once the phase mask splits the light, the two first order diffraction beams are guided to the optical fiber by two mirrors mounted to rotational stages. Bragg grating wavelength control is done by adjusting one of the mirrors to change the angle at which the two UV beams interfere.

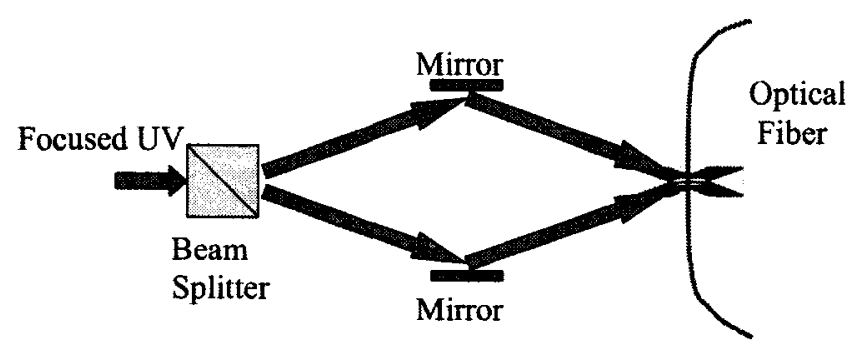

Figure 5.4: Interferometric technique.

\section{Post processing of Bragg grating}

Over time, the average refractive index of the Bragg grating decreases causing a shift in the Bragg wavelength. To prevent this from happening during testing the FBG must be annealed. Typical standard annealing bakes the Bragg gratings in an oven for 4 hours at $300 \mathrm{~F}$.

Figures 5.5 on the next page and 5.6 on the following page show the FBG writing processes at the Carleton Laboratory for Laser Induced Photonic Structures (CLLIPS), while Figure 5.7 on page 96 shows the phase mask that has been used in the writing process.

\subsubsection{The Implemented FBG Characteristics}

Figure 5.8 on page 97 shows the implemented FBG reflection and transmission characteristics. This FBG will be designated as FBG1 throughout this work. FBG1 has its peak 


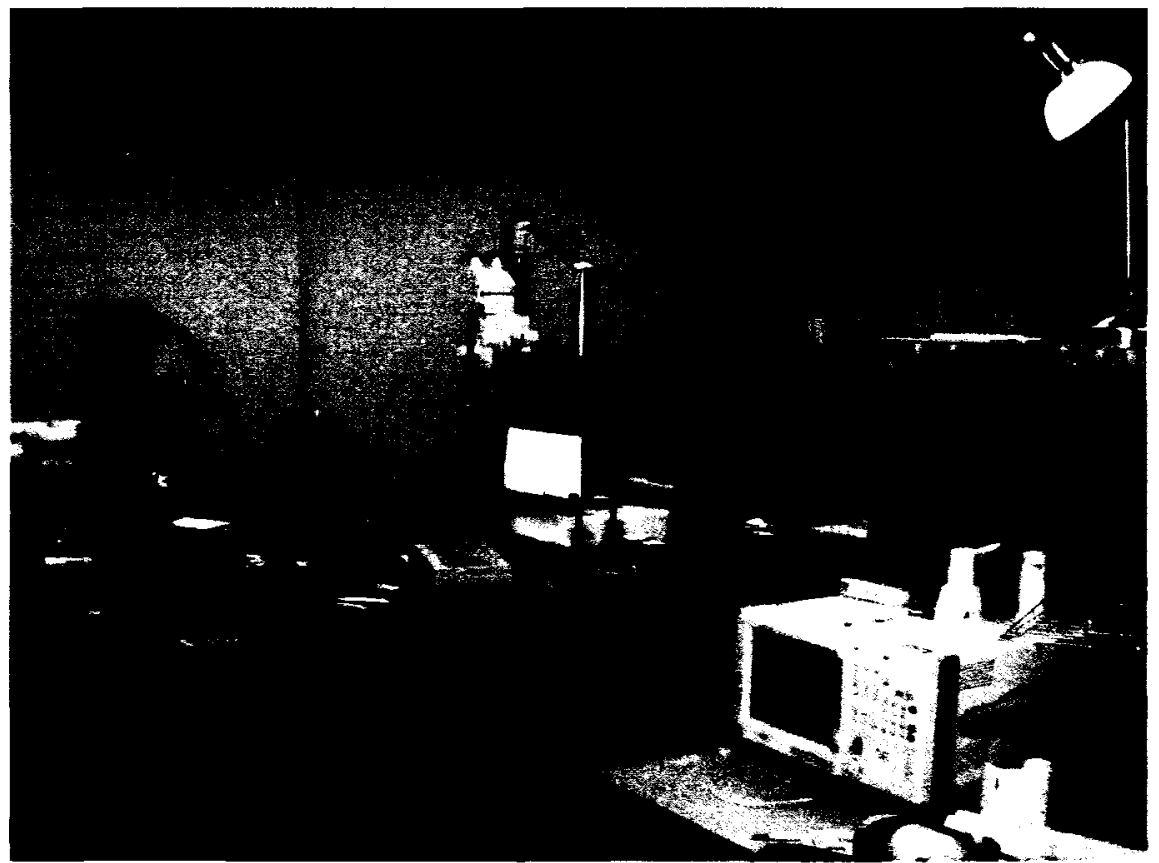

Figure 5.5: Different equipments used in the implementation of FBG at CLLIPS.

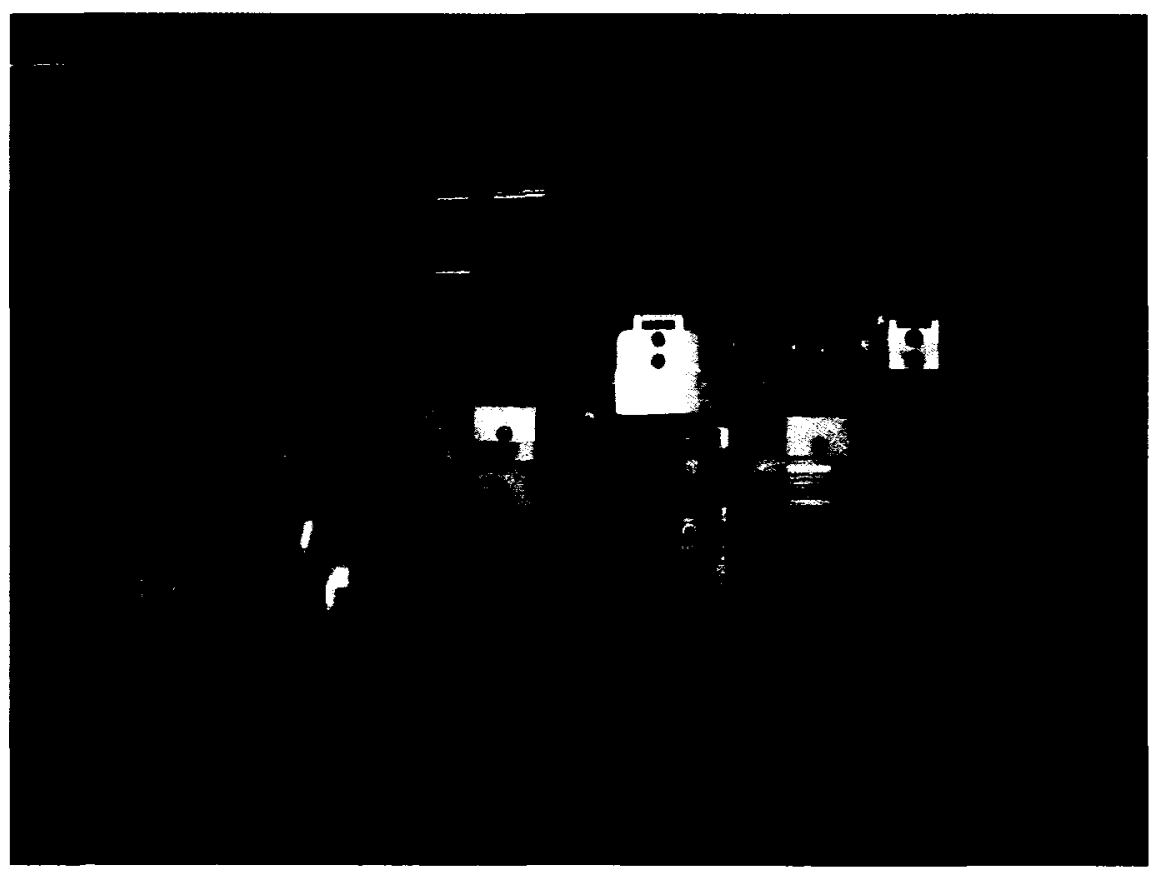

Figure 5.6: FBG writing process at CLLIPS. 


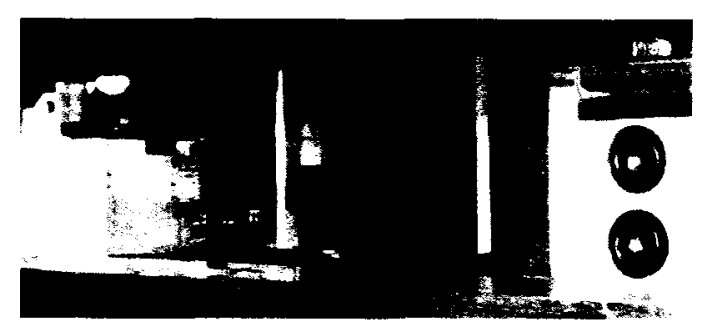

Figure 5.7: The phase mask used in FBG implementation at CLLIPS.

reflectivity of 0.2166 at wavelength $1552.649 \mathrm{~nm}$, the measured 3-dB bandwidth of FBG1 is $0.914 \mathrm{~nm}$.

The other implemented FBGs characteristics are shown in Appendix B. The performance of DFB laser diode attached to FBG will be discussed in Chapter 6 . 
2025 Dec $1910: 09$

\begin{tabular}{|c|c|}
\hline 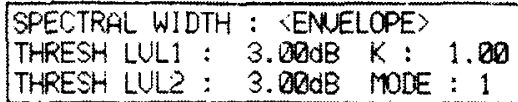 & $\begin{array}{rlr}\Delta k & : 0.914 \mathrm{~nm} \\
h c & : 1552,649 \mathrm{~mm}\end{array}$ \\
\hline
\end{tabular}

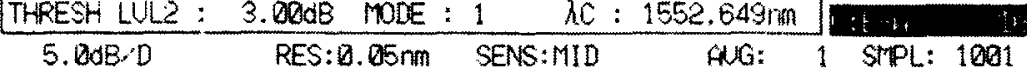
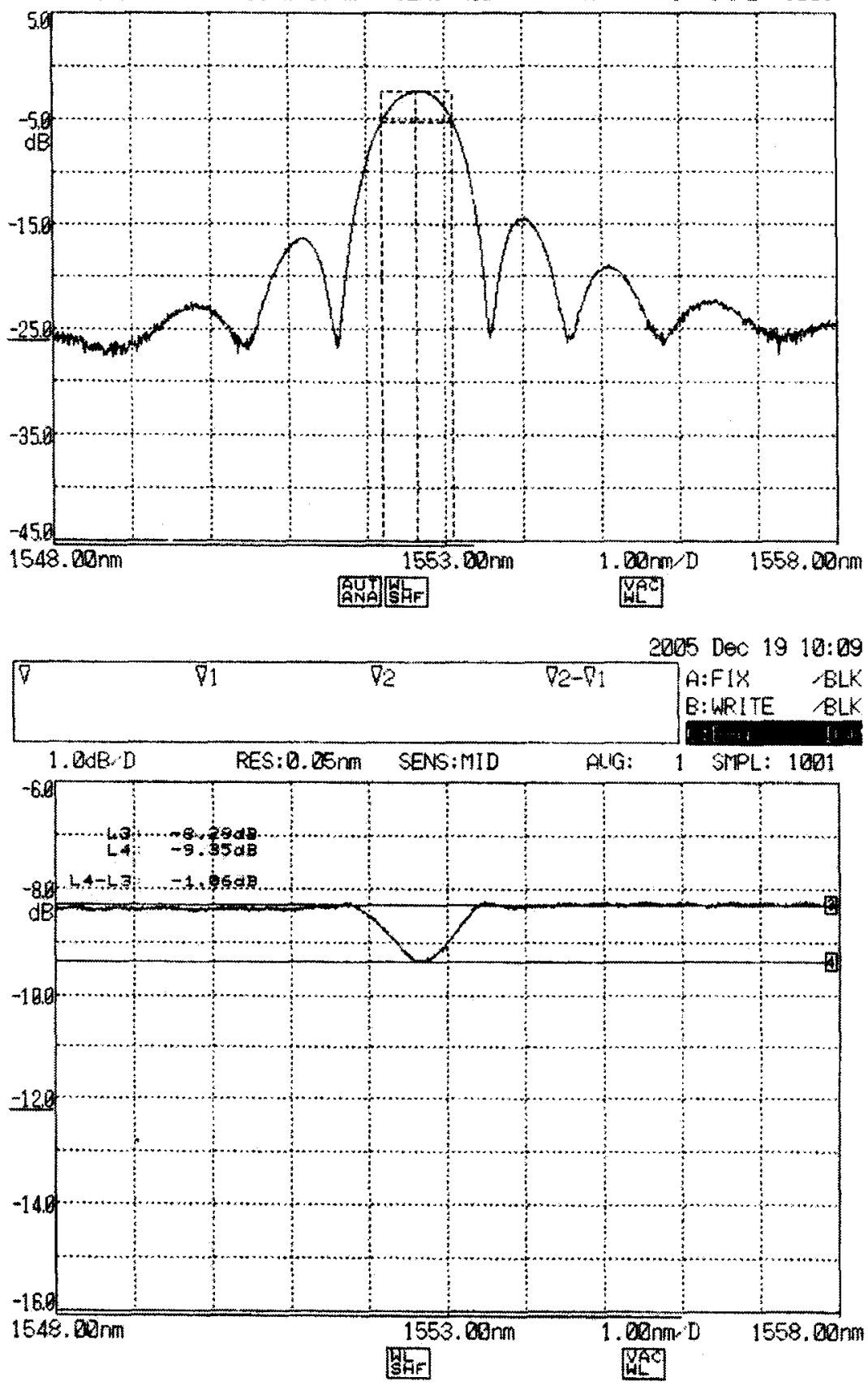

Figure 5.8: The implemented FBG1 reflection (top) and transmission (bottom) characteristics. 


\subsection{Summary}

In this chapter we presented the design and implementation of FBG. This design is based on the laser output characteristics, and the back-reflection level required to drive the laser to operate in feedback Regime V. Analytical formulas used to determine the design parameters were discussed. OptiGrating design software was used to design the desired FBG. Implementation procedures for FBG at CLIPS are presented at the end of chapter. 


\section{Chapter 6}

\section{DFB Laser Diode with External FBG}

In this chapter, a feedback topology is proposed that advantageously applies optical feedback to the DFB laser in order to enable the omission of the isolator without significantly degrading the performance of the DFB laser. The proposed topology is achieved by attaching a FBG to a DFB laser. FBG characteristics were chosen such that the attached DFB laser would be driven to operate in feedback Regime V. Several FBGs have been implemented and a variety of DFB laser modules were used to experimentally determine the DFB laser diode performance when attached to a FBG. Table 6.1 summarizes the different components used in this work along with their affiliated notations.

\begin{tabular}{|c|c|c|}
\hline Notation & Description & Manufacturer \\
\hline \hline FBG1 & FBG with $R=0.2166$ at $\lambda=1552.649 \mathrm{~nm}$ & CLLIPS \\
\hline FBG2 & FBG with $R=0.2773$ at $\lambda=1552.698 \mathrm{~nm}$ & CLLIPS \\
\hline FBG4 & FBG with $R=0.2112$ at $\lambda=1552.653 \mathrm{~nm}$ & CLLIPS \\
\hline FBG7 & FBG with $R=0.50$ at $\lambda=1553.231 \mathrm{~nm}$ & CLLIPS \\
\hline FBG10 & FBG with $R=0.25$ at $\lambda=1552.505 \mathrm{~nm}$ & CLLIPS \\
\hline FBG11 & FBG with $R=0.293$ at $\lambda=1552.83 \mathrm{~nm}$ & Avensys \\
\hline FBG12 & FBG with $R=0.2897$ at $\lambda=1313.19 \mathrm{~nm}$ & Avensys \\
\hline DFB1 & FLD3F7CZ 1310 nm isolated butterfly DFB laser diode (LD) & Eudyna (Fujitsu) \\
\hline DFB2 & ES/FLD3F7CZ557-MX09081 1310 nm unisolated DFB LD & Eudyna (Fujitsu) \\
\hline DFB3 & ES/FLD3F7CZ557-MX09082 1310 nm unisolated DFB LD & Eudyna (Fujitsu) \\
\hline DFB4 & T13D-PFA2-C1 1310 nm unisolated coaxial DFB LD & Laser Mate \\
\hline DFB5 & T15D-PFA2-C1 1550 nm unisolated coaxial DFB LD & Laser Mate \\
\hline
\end{tabular}

Table 6.1: Various components used in measurements.

The DFB laser diodes have to be biased properly in order to emit around the central 
wavelength of the attached FBG, hence, achieving the back-reflection required for the feedback Regime $\mathrm{V}$ operation. The performance of the proposed feedback topology is analyzed in the remainder of this chapter. The spectrum of the DFB laser diode attached to the FBG was examined; the RIN and distortion of the combined configuration was measured. Results of the proposed configuration was also compared to that of the isolated DFB laser.

\subsection{Spectrum Stability}

Previously reported research discuss using FBGs to force a laser into Regime IV [58]. This regime is mostly applicable to low-speed digital signaling applications and erbiumdoped fiber amplifiers pumping. For CATV applications, operation in Regime V is highly recommended. This section will show how the use of an FBG stabilized the intensity and wavelength fluctuations of an unisolated DFB laser. This was due to operation in feedback Regime V.

\subsection{1 $1550 \mathrm{~nm}$ wavelength range}

FBG1 was attached to DFB5. The reflection and transmission characteristics of FBG1 are found in Figure 5.8 on page 97 . The DFB5 spectrum shows high intensity and wavelength stability with narrow linewidth. This is contributed to the induced optical back-reflections by FBG1. Figure 6.1 on the next page shows the resulting spectrum for a $15 \mathrm{~mA}$ bias current.

FBG2 reflection and transmission characteristics are shown in Figure B.7 on page 157. When FBG2 was attached to DFB5 and biased at $15 \mathrm{~mA}$ in order to emit around the central wavelength of the FBG2, the resulting output spectrum exhibited a stable narrow linewidth operation. This is shown in Figure 6.2 on the next page.

DFB5 also achieved stable spectra when attached to all other FBGs. These results are shown in Appendix B.

The measured DFB laser spectra shown in Figure 5.2 on page 92 shows that as the bias current increases the laser emission wavelength shifts towards higher values. Optical reflections from FBG reduces the laser threshold level [58], which is equivalent to an increase 


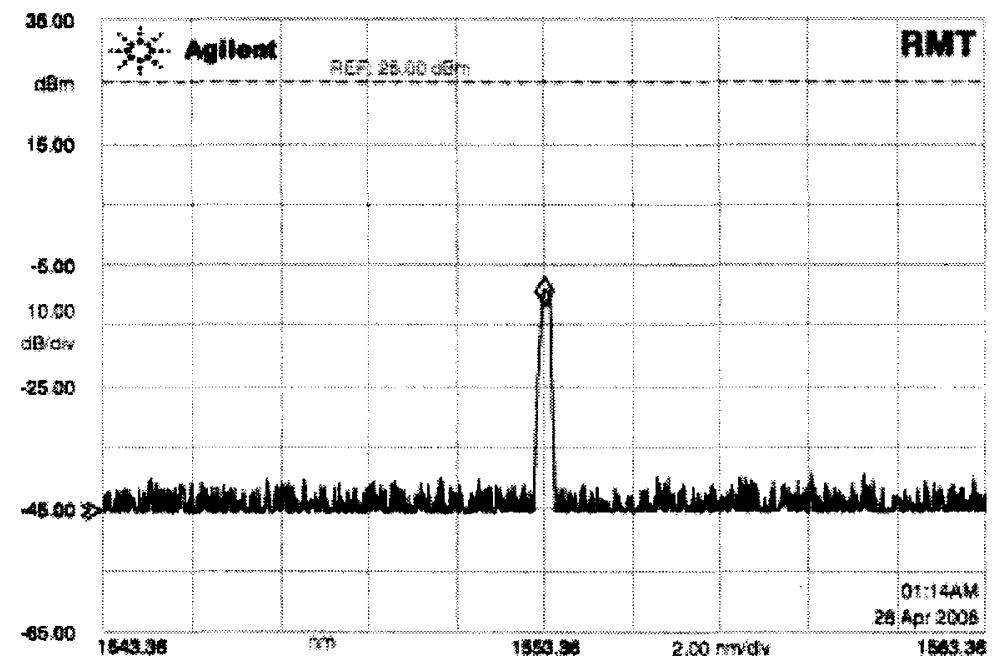

Figure 6.1: Spectrum of DFB5 attached to FBG1 and operating at a bias level of $15 \mathrm{~mA}$.

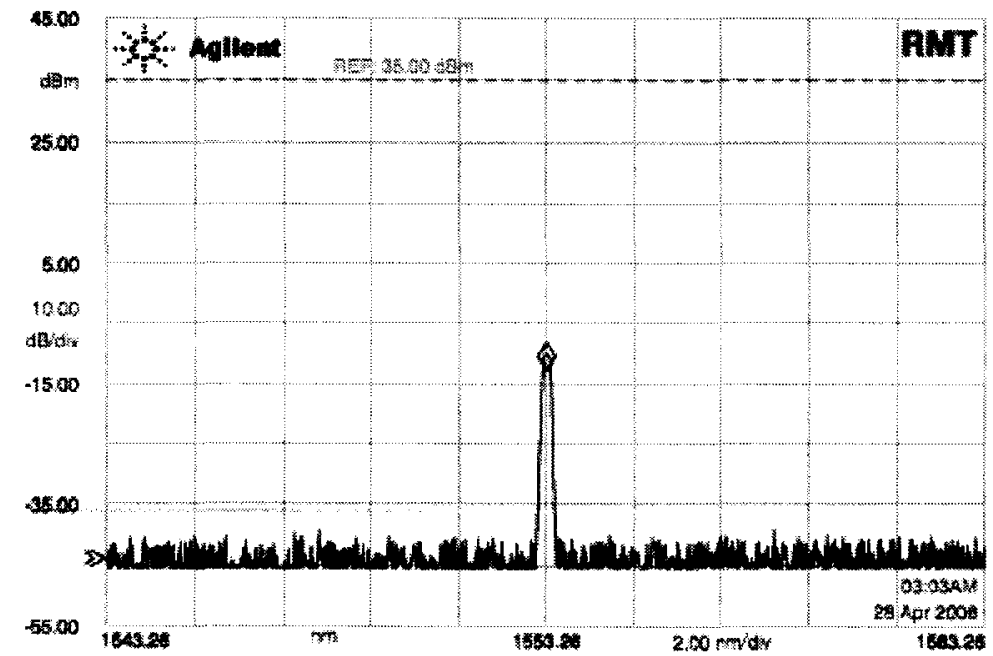

Figure 6.2: Spectrum of DFB5 attached to FBG2 and operating at a bias level of $15 m A$. 
in the laser's bias current. This can explain why there is a wavelength shift in the laser output when the FBG is attached.

\subsection{2 $1310 \mathrm{~nm}$ wavelength range}

FBG12 has been spliced to DFB2 to minimize connector losses as well as improve the coupling efficiency between the laser diode and the FBG. FBG12 shown in Figure B.12 has a peak reflectivity of 0.2897 , lies at $1313.19 \mathrm{~nm}$ and has a bandwidth (FWHM) of $0.45 \mathrm{~nm}$.

When DFB2 was biased properly to emit at FBG12 peak reflectivity wavelength, that is $1313.19 \mathrm{~nm}$, the reflection was strong enough to drive DFB2 to operate in the stable feedback Regime $\mathrm{V}$ as shown in Figure 6.3.

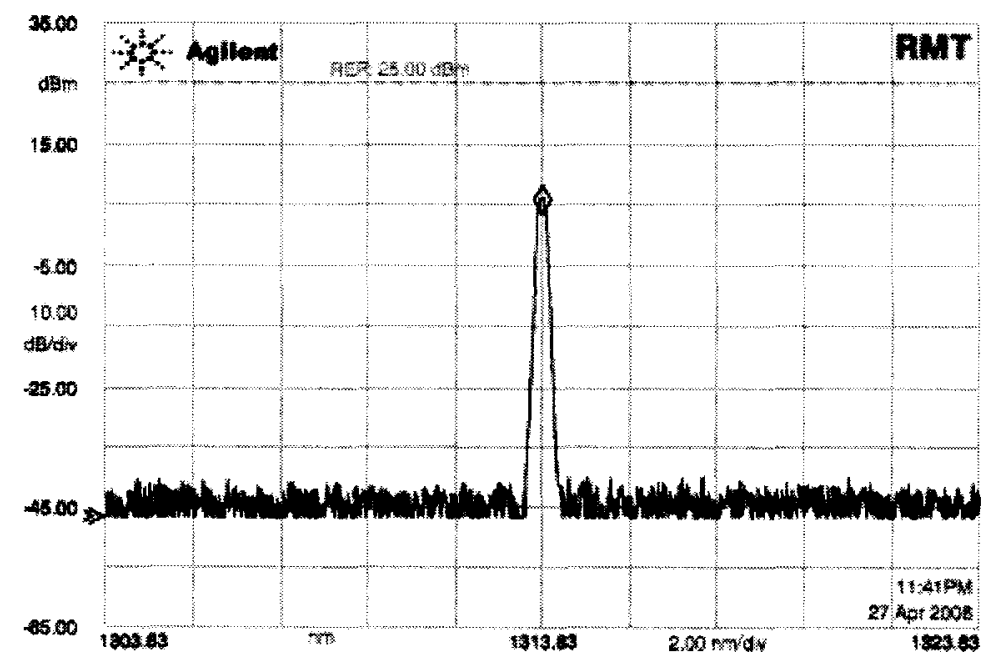

Figure 6.3: Spectrum of DFB2 attached to FBG12 and operating at stable feedback Regime V.

When DFB2 laser was biased to emit at $1313 \mathrm{~nm}$, a strong back-reflection from FBG12 was achieved. However, the level of the back-reflection was less than that required to achieve feedback Regime V operation. Hence, DFB2 was driven to operate in the coherence collapse regime (Regime IV), in which an unstable operation for DFB2 with broadened linewidth was observed. Figure 6.4 on the following page shows the resulting spectrum. 


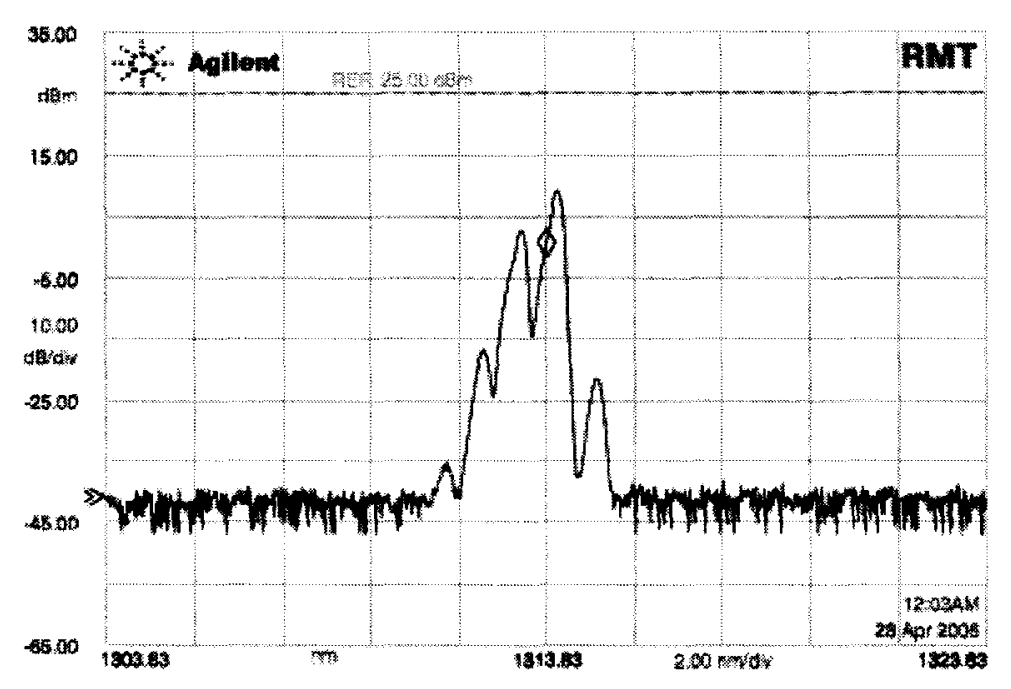

Figure 6.4: Spectrum of DFB2 attached to FBG12 and operating at unstable feedback Regime IV.

\subsection{Relative Intensity Noise Measurements}

Carrier-to-Noise Ratio (CNR) is defined as the ratio of the carrier to the total noise power in $4 \mathrm{MHz}$ bandwidth. Analog AM-VSB video CATV signals require a CNR near $50 \mathrm{~dB}$ for acceptable picture quality. This CNR is much larger than the $20 \mathrm{~dB}$ required for digital and FM systems [4]. RIN from the laser is one of the major factors that limits the CNR. Low RIN is highly recommended for the DFB lasers in order to achieve the desired CRN for analog CATV systems. This section shows how low RIN can be achieved by attaching an FBG to the DFB laser diode.

\subsection{1 $1550 \mathrm{~nm}$ wavelength range}

Figure 6.5 on the next page shows the measured average RIN of DFB5 biased at $15 \mathrm{~mA}$. Figure 6.6 on the following page shows the measured average RIN of DFB5 attached to a $30 \mathrm{~dB}$ optical isolator and biased at the same level. The RIN was measured at $1 \mathrm{GHz}$ frequency offset. Using optical isolator decreases the average RIN from $-108.96 \mathrm{~dB} / \mathrm{Hz}$ to $-114.51 \mathrm{~dB} / \mathrm{Hz}$.

Figure 6.7 on page 105 shows the measured average RIN of DFB5 biased at $15 \mathrm{~mA}$, 


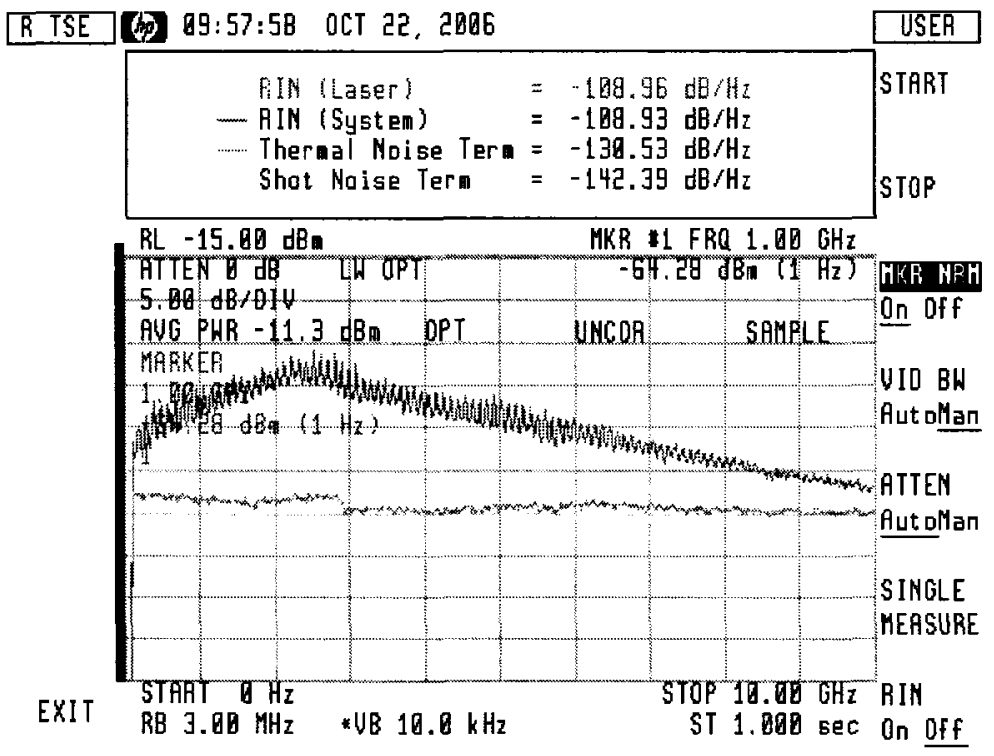

Figure 6.5: Measured average RIN of DFB5 operating at a bias level of $15 m A$.

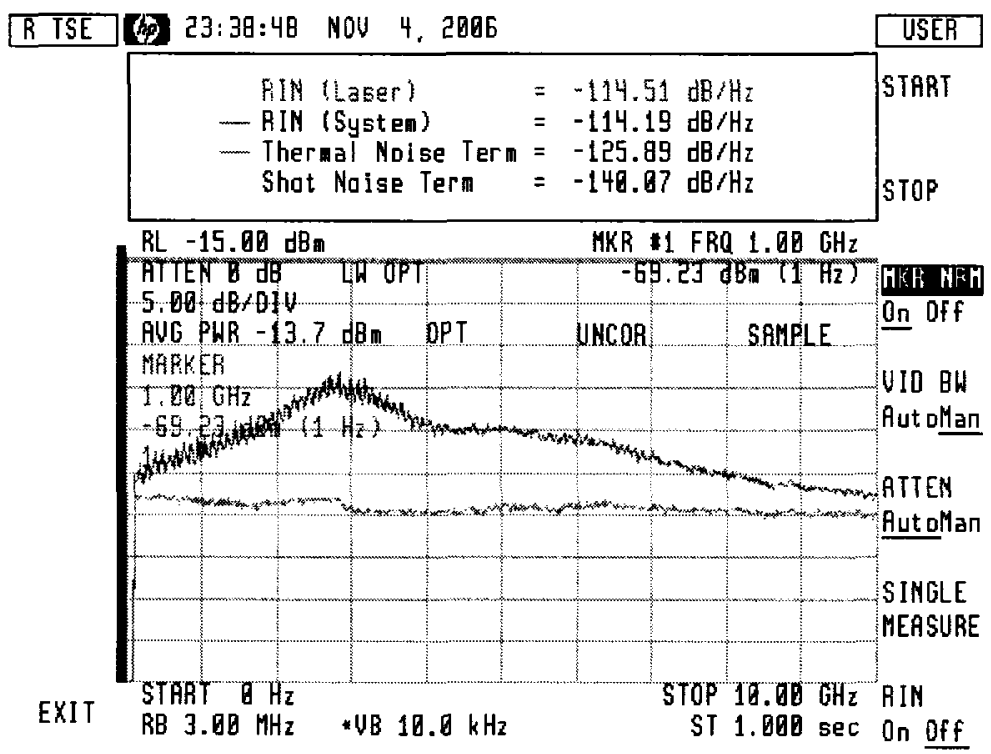

Figure 6.6: Measured average RIN of DFB5 attached to a $30 \mathrm{~dB}$ optical isolator, and operating at a bias level of $15 \mathrm{~mA}$. 
with FBG10 attached to DFB5. The measured average RIN at a $1 \mathrm{GHz}$ frequency offset is $-115.13 \mathrm{~dB} / \mathrm{Hz}$.

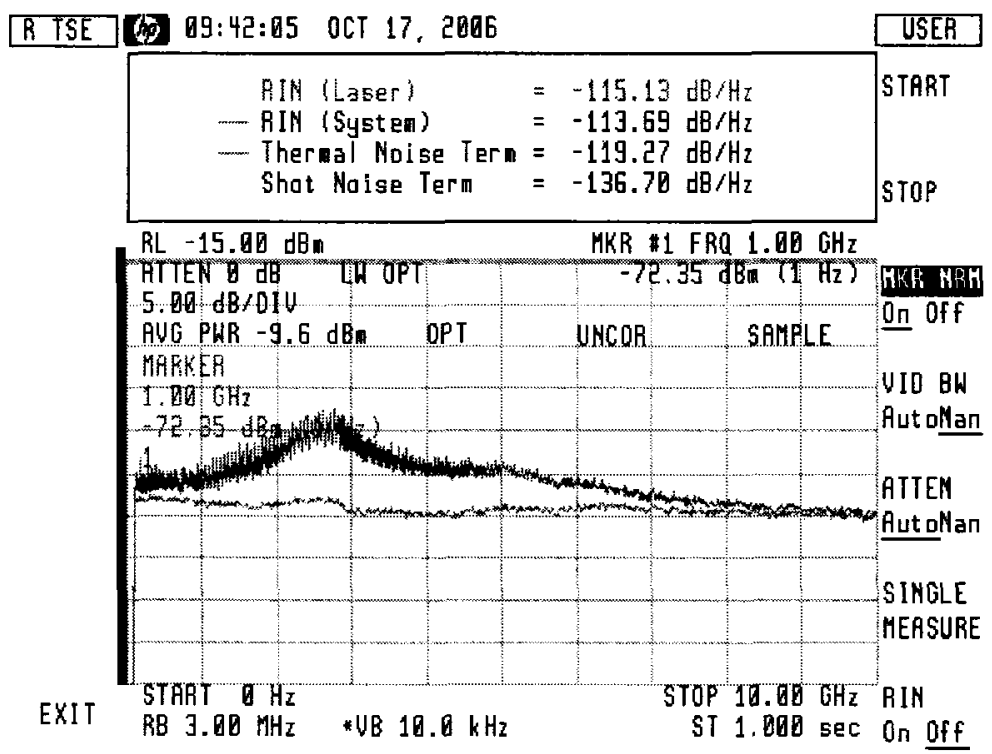

Figure 6.7: Measured average RIN of DFB5 attached to FBG10, and operating at a bias level of $15 \mathrm{~mA}$.

Figure 6.8 on the following page shows the measured average RIN of DFB5 biased at $15 \mathrm{~mA}$, with FBG11 attached to DFB5. The measured average RIN at a $1 \mathrm{GHz}$ frequency was $-117.31 \mathrm{~dB} / \mathrm{Hz}$. Average RIN measured for DFB5 attached to other FBGs is shown in Appendix B.

Table 6.2 on the next page summarizes the above measured average RIN for the various DFB5 configurations.

Results show that using FBG1, FBG2, or FBG10 improves the unisolated DFB5's RIN by an average of $7 \mathrm{~dB} / \mathrm{Hz}$. This improvement is comparable to the improvement obtained using a $30 \mathrm{~dB}$ optical isolator. However, using FBG11 achieves better RIN while maintaining the same bias current level. 


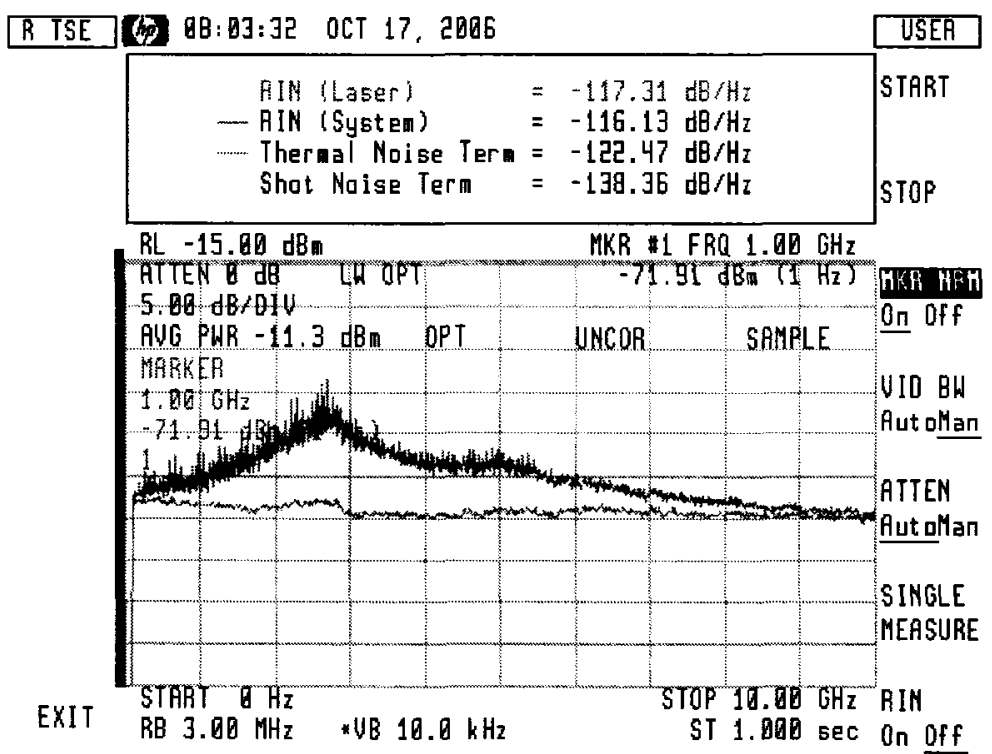

Figure 6.8: Measured average RIN of DFB5 attached to FBG11, and operating at a bias level of $15 m A$.

\begin{tabular}{|c|c|c|}
\hline Configuration & FBG reflectivity & measured RIN (dB/Hz) \\
\hline \hline Unisolated DFB5 & - & -108.96 \\
\hline DFB5 with 30-dB isolator & - & -114.51 \\
\hline FBG1 attached to DFB5 & 0.2166 & -114.02 \\
\hline FBG2 attached to DFB5 & 0.2737 & -115.99 \\
\hline FBG10 attached to DFB5 & 0.25 & -115.13 \\
\hline FBG11 attached to DFB5 & 0.293 & -117.31 \\
\hline
\end{tabular}

Table 6.2: Measured average RIN for different DFB5 configurations. 


\subsection{2 $1310 \mathrm{~nm}$ wavelength range}

Figures 6.9 and 6.10 on the following page respectively show the average RIN measurements for DFB2 attached to FBG12, and for DFB1 with a built-in optical isolator. DFB2 attached to FBG12 achieved an average RIN level of $-158.49 \mathrm{~dB} / \mathrm{Hz}$. That is comparable to an average RIN level of $-157.87 \mathrm{~dB} / \mathrm{Hz}$ of DFB1 at a $1 \mathrm{GHz}$ frequency offset and same average optical power (i.e. $5.1 \mathrm{dBm}$ ) [54].

Two observations are noteworthy. Firstly, although it is known that the laser RIN has its maximum near the relaxation oscillation frequency, this is not witnessed in the measurement plots. This is due to the fact that the relaxation frequency of the laser at this bias level is much more than the stop frequency of the measurement (i.e, $5 \mathrm{GHz}$ ). Secondly, the drop that exists at a frequency $2.9 \mathrm{GHz}$ is due to the fact that the Agilent $71400 \mathrm{C}$ lightwave signal analyzer uses two different tracking generator modules to cover the full frequency range from $20 \mathrm{~Hz}$ to $18 \mathrm{GHz}$. The first module covers frequencies ranging from $20 \mathrm{~Hz}$ to $2.9 \mathrm{GHz}$, while the second covers frequencies ranging from $2.7 \mathrm{GHz}$ to $18 \mathrm{GHz}$. The drop that exists at a frequency of $2.9 \mathrm{GHz}$ is due to the transition from the first module to the second.

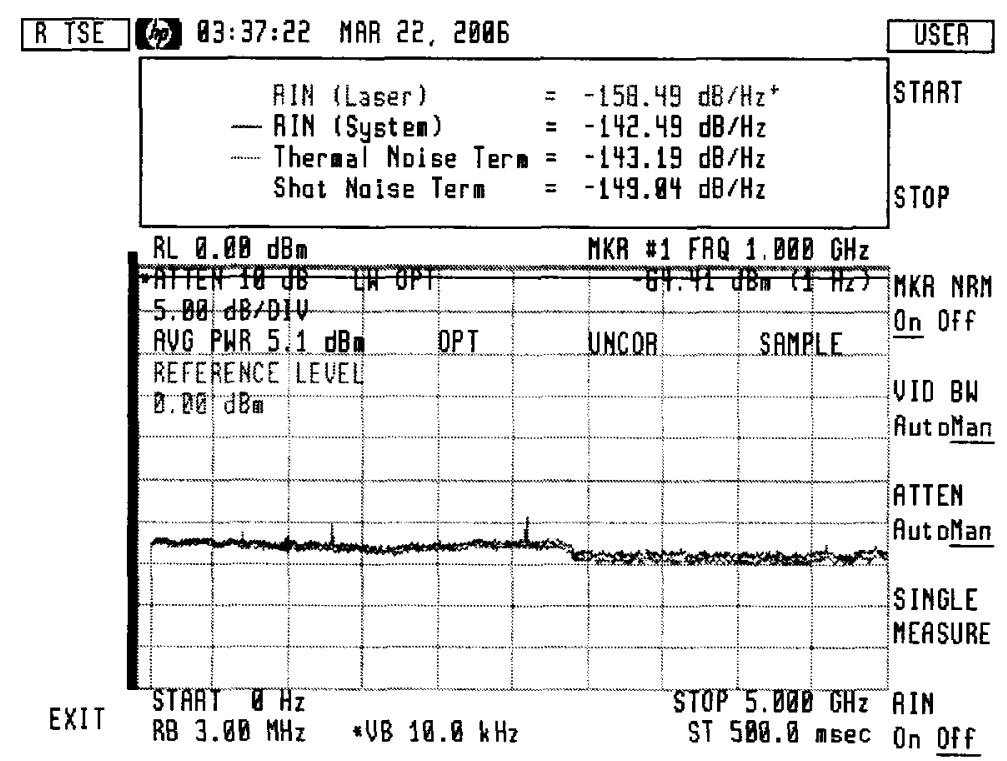

Figure 6.9: Measured RIN of DFB2 attached to FBG12. 


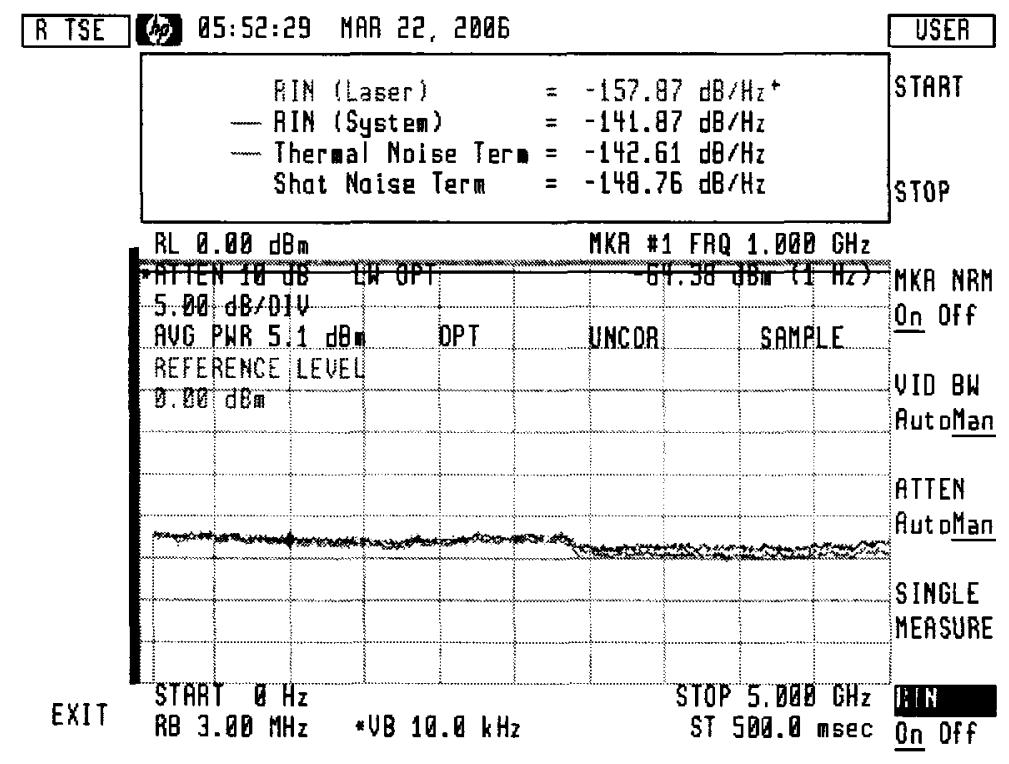

Figure 6.10: Measured RIN for DFB1.

\subsection{Distortion Measurements}

In addition to the high CNR recommended for analog CATV systems, low CSO and CTB are also desirable in order to achieve high analog CATV signal quality. CSO is defined as the ratio of the carrier to the total power within the largest accumulation of second-order distortion products within each channel. CTB is similarly defined as the ratio of carrier to total power within the largest accumulation of third-order distortion products within each channel. This section shows how attaching a FBG to a DFB laser affects the second and third order harmonic distortions of the DFB laser.

\subsection{1 $1550 \mathrm{~nm}$ wavelength range}

Figures 6.11 to 6.14 on page 111 show the measured distortion of DFB5 attached to various tested FBGs as a function of bias current. Measured distortion for DFB5 both with and without an isolator were also included on the figures for the sake of comparison. Distortion measurement results for the other tested FBGs are included in Appendix B. 
Figure 6.11 shows the measured second order harmonic distortion for FBG1 attached to DFB5 operating at a carrier frequency of $500 \mathrm{MHz}$ and a power of $0 \mathrm{dBm}$. Figure 6.12 shows the results of the third-order harmonic distortion for the same setup.

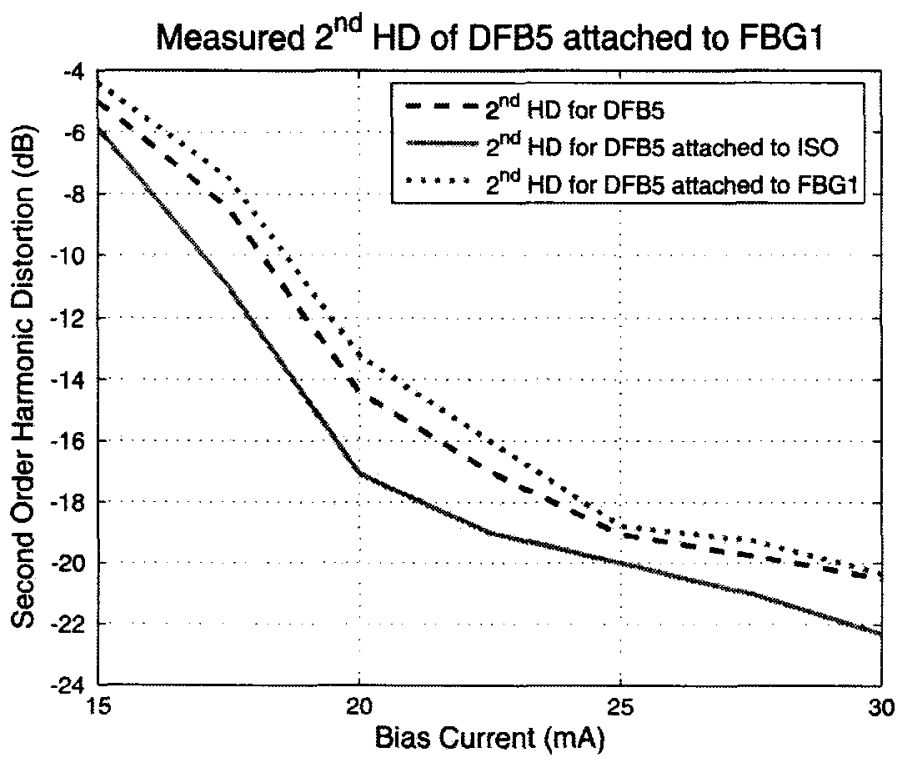

Figure 6.11: Measured second order harmonic distortion of FBG1 attached to DFB5 normalized to the fundamental frequency .

Figure 6.13 and Figure 6.14 on page 111 show the measured second and third order harmonic distortions for FBG2 attached to DFB5 operating at a carrier frequency of $500 \mathrm{MHz}$ and a power of $0 \mathrm{dBm}$.

Results demonstrate that both second and third order harmonic distortions are decreasing functions of the bias current. Moreover, compared to isolated DFB laser, using FBGs increases both second and third order harmonic distortions by less than $5 \mathrm{~dB}$ compared to the isolated DFB. The above results are summarized in Table 6.3 on page 111.

\subsection{2 $1310 \mathrm{~nm}$ wavelength range}

Figure 6.15 on page 112 and Figure 6.16 on page 113 show the measured distortions as a function of the bias current for FBG12 attached to DFB2.

Figure 6.15 on page 112 shows the measured second order harmonic distortions for 


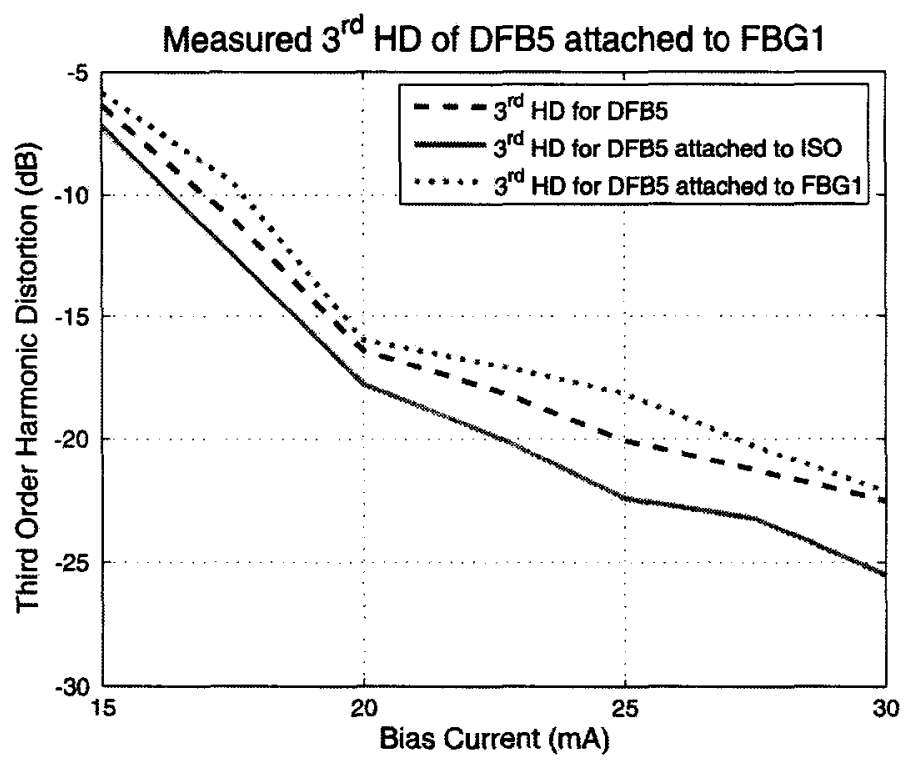

Figure 6.12: Measured third order harmonic distortion of FBG1 attached to DFB5 normalized to the fundamental frequency.

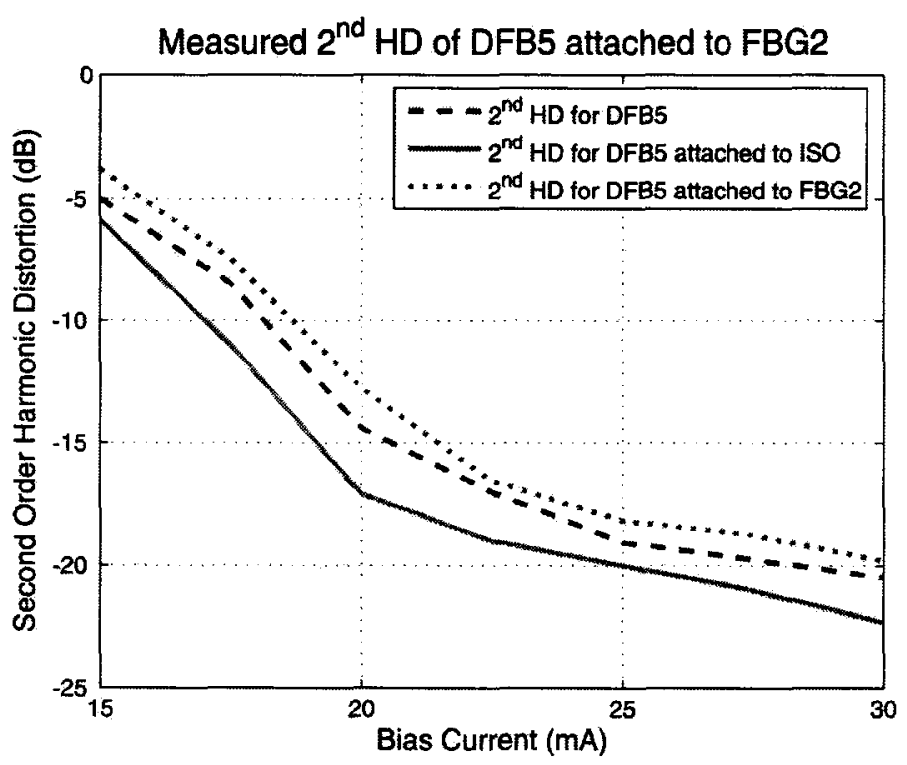

Figure 6.13: Measured second order harmonic distortion of FBG2 attached to DFB5 normalized to the fundamental frequency. 


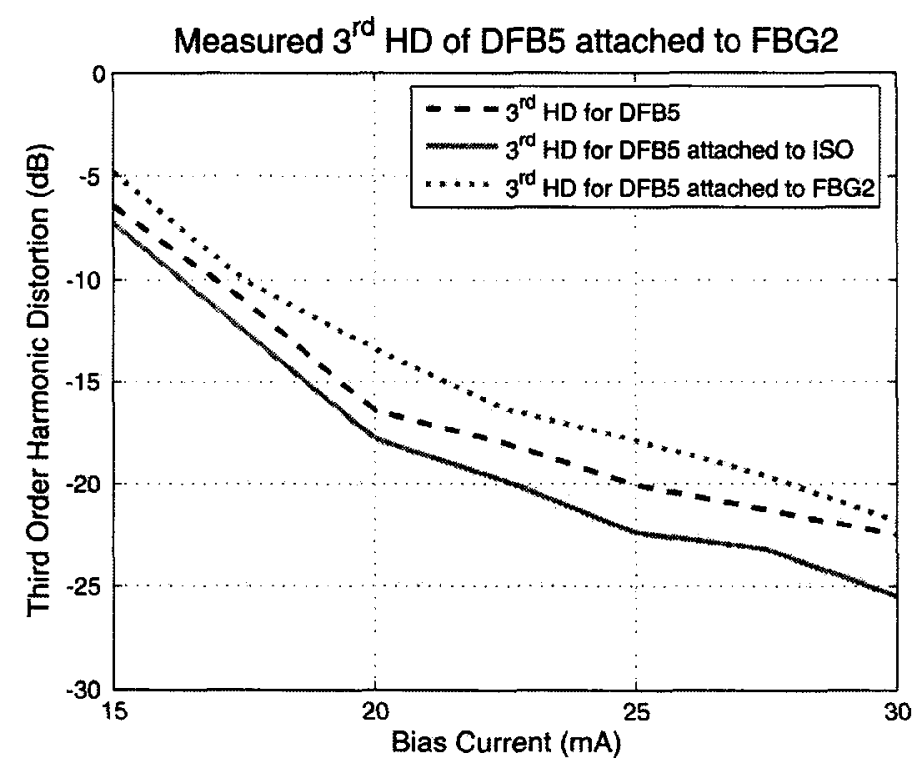

Figure 6.14: The measured third order harmonic distortion of FBG2 attached to DFB5 normalized to the fundamental frequency.

\begin{tabular}{|c|c|c|c|}
\hline Configuration & FBG reflectivity & $2^{\text {nd }}$ HD (dB) & $3^{\text {rd }}$ HD (dB) \\
\hline \hline Unisolated DFB5 & - & -19.06 & -20.06 \\
\hline DFB5 with 30-dB isolator & - & -20 & -22.01 \\
\hline FBG1 attached to DFB5 & 0.2166 & -18.78 & -18.15 \\
\hline FBG2 attached to DFB5 & 0.2737 & -18.17 & -17.87 \\
\hline FBG4 attached to DFB5 & 0.2112 & -17.8 & -19.7 \\
\hline FBG7 attached to DFB5 & 0.50 & -17.1 & -19.7 \\
\hline FBG10 attached to DFB5 & 0.25 & -18.4 & -19.5 \\
\hline FBG11 attached to DFB5 & 0.293 & -18.4 & -19.1 \\
\hline
\end{tabular}

Table 6.3: Measured distortion for various DFB5 configurations (bias current $=25 \mathrm{~mA}$ ). 
FBG12 attached to DFB2 operating at a carrier frequency of $500 \mathrm{MHz}$ and a power of $0 \mathrm{dBm}$. Figure 6.16 on the following page shows the measured third order harmonic distortion. Equivalent measurements for DFB1 were included in the figures for comparison purposes.

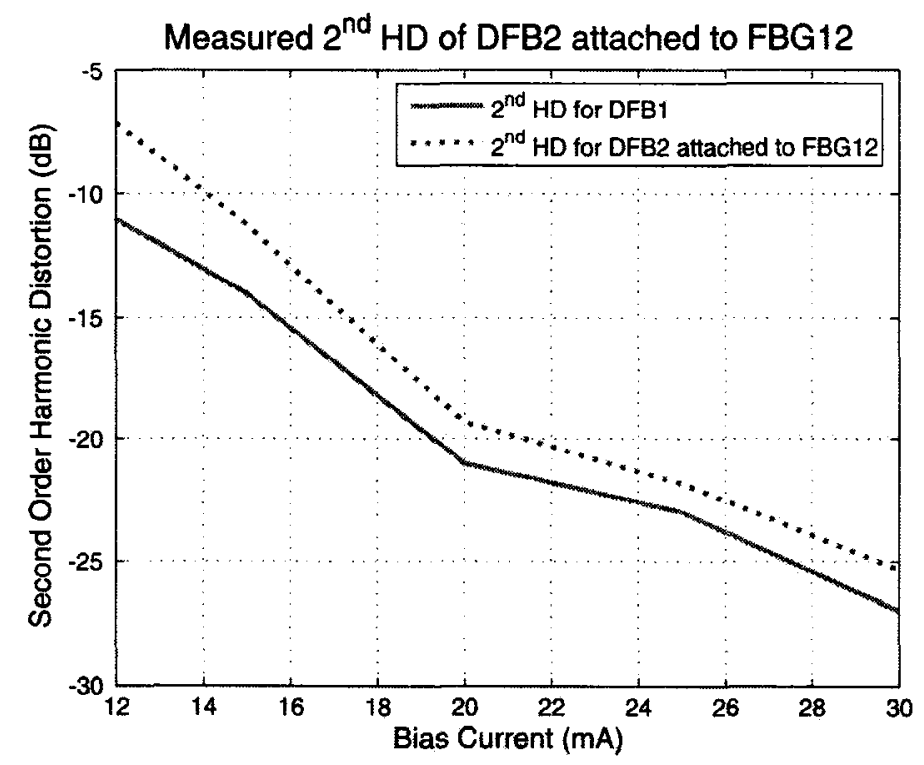

Figure 6.15: Measured second order harmonic distortion of DFB2 attached to FBG12 normalized to the fundamental frequency.

These results show that both second and third order harmonic distortion are decreasing functions of the bias current. Moreover, using FBGs has slightly increased the lasers' distortions. 


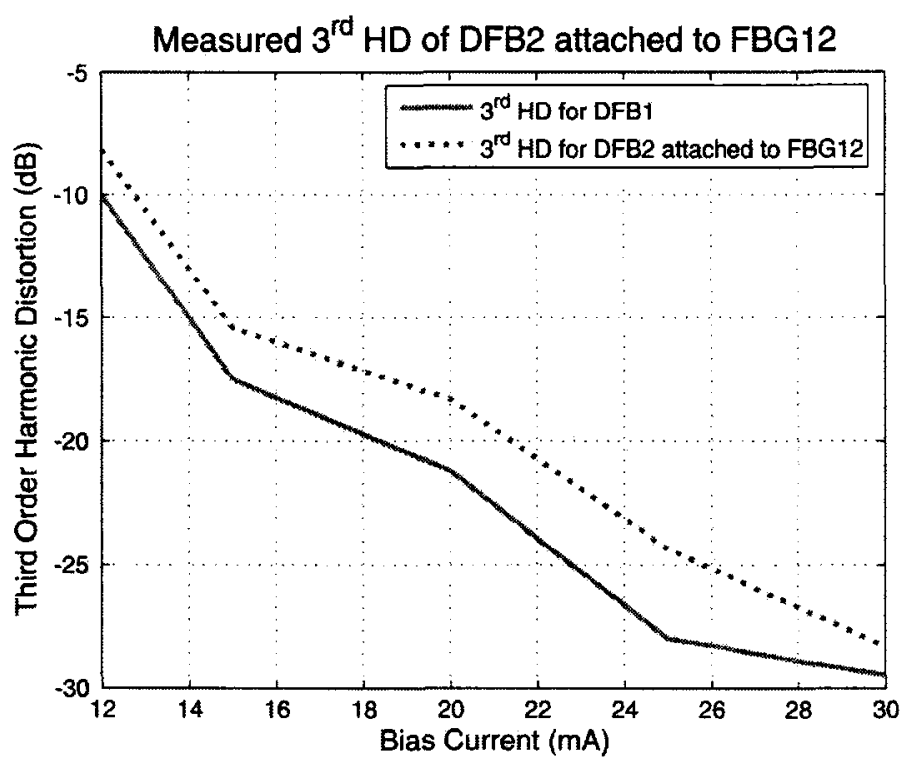

Figure 6.16: Measured third order harmonic distortion of DFB2 attached to FBG12 normalized to the fundamental frequency.

\subsection{Summary}

Using FBG stabilizes DFB lasers and improve their RIN. All tested DFB laser diodes showed a stable spectrum, low RIN, and narrow linewidth output when attached to the implemented FBGs. Performance of DFB lasers attached to appropriate FBGs were comparable to that of isolated DFB lasers. When feedback Regime V operation is reached, increasing FBG reflectivity decreases the RIN. However, this may come at the expense of lower output optical power. Furthermore, including a FBG has increased the DFB lasers' distortions by around $3 \mathrm{~dB}$ compared to isolated DFB lasers. Lasers' nonlinearity compensation will be discussed in detail in Chapter 7 .

Using optical isolator can improve the unisolated laser noise and linearity performance, on the other hand it increases cost and complicate the construction of the laser module. Extra time is required to optically align the isolator, additionally it cannot guarantee complete removal for the optical back-reflections. In this chapter we showed that using an unisolated DFB laser attached to a FBG and operating in feedback Regime $\mathrm{V}$ achieves noise and linearity performance that is comparable to that of the isolated DFB laser. 


\section{Chapter 7}

\section{Inverse Laser Model Including Feedback Derivation and Modeling}

\subsection{Introduction}

The laser is a non-linear system; it produces both even and odd order harmonic distortion. To meet the restrictions imposed on the DFB lasers for analog CATV systems, the laser's distortion should be kept at a minimum. Fabrication of high linearity laser is an expensive task and requires extensive screening that may result in low yields. The other alternative is to use one of the linearization techniques. Several linearization techniques were reviewed in [19] these included: optical feedback and optical feed-forward [59-63], post-distortion [64-66], pre-distortion [67-72], adaptive pre-distortion [73-76], external light injection [77], and cascaded linearized modulator [78]. The analysis made in [19] in terms of performance, cost, and suitability for analog CATV systems supported the use of pre-distortion techniques.

In this chapter a laser pre-distorter will be developed based on the inverse laser Volterra model including feedback effect. The rate equations that describing the original laser model will be algebraically manipulated in order to get the laser driving current as a function of the laser output power, then third order Volterra transfer functions will be extracted from these equations representing the pre-distorter Volterra model. The third order predistorter Volterra model will be implemented in ADS and cascaded to the original laser model to reduce the overall distortion and hence keep the Composite Second Order (CSO) and Composite Triple Beat (CTB) at lower levels. 


\subsection{Inverse Laser Model Including Feedback Deriva- tion}

When the distortion is frequency dependent in a non-linear system, the nonlinearity can be represented by a Volterra functional series (a power series with memory) $[79,80]$, instead of a Taylor series (a power series without memory). The coefficients will be extracted using Volterra transfer functions in order to determine the laser inverse function by deriving the Volterra series of $I=f(P)$, where $I$ is the injection current, and $P$ is the photon density.

The laser diode rate equations including feedback term are given by the following two relations:

$$
\frac{d P_{n}(t)}{d t}=\frac{\left(C_{n}(t)-C_{t r}^{n}\right)}{\tau_{p h}\left(1-C_{t r}^{n}\right)} \frac{P_{n}(t)}{\sqrt{1+P_{r}^{n}}}-\frac{P_{n}(t)}{\tau_{p h}}+\beta_{s p} C_{n}(t)+\frac{2}{\tau_{L}} \kappa_{e x t} P_{n}(t) \cos \left(\omega_{t h} \tau_{e x t}\right)
$$

and

$$
\frac{d C_{n}(t)}{d t}=\frac{I(t)}{q V_{c} C_{t h}}-\frac{\left(C_{n}(t)-C_{t r}^{n}\right)}{\tau_{e} \tau_{p h}\left(1-C_{t r}^{n}\right)} \frac{P_{n}(t)}{\sqrt{1+P_{r}^{n}}}-\frac{C_{n}(t)}{\tau_{e}}
$$

The different parameters of (7.2.1) and (7.2.2) were defined in the Chapter 3. To get $I=$ $f(P)$ the laser diode rate equations should be denormalized and algebraically manipulated. From (7.2.1) the carrier density is given as,

$$
C=\frac{\frac{d P}{d t}+\frac{P}{\tau_{p h}}+\frac{G C_{t r} P}{\sqrt{1+P / P_{s}}}-\frac{2}{\tau_{L}} \kappa_{e x t} P \operatorname{Cos}\left(\omega_{t h} \tau_{e x t}\right)}{\frac{\Gamma \beta_{s p}}{\tau_{e}}+\frac{G P}{\sqrt{1+P / P_{s}}}}
$$

Expressing $P$ and $C$ in terms of their DC and AC components, i.e, $P=P_{d c}+P_{a c}$ and $C=C_{d c}+C_{a c}$ in (7.2.3) yields,

$$
C_{d c}+C_{a c}=\frac{P^{\prime}+\frac{\left(P_{d c}+P_{a c}\right)}{\tau_{p h}}+\frac{G C_{t r}\left(P_{d c}+P_{a c}\right)}{\sqrt{1+\left(P_{d c}+P_{a c}\right) / P_{s}}}-\frac{2}{\tau_{L}} \kappa_{e x t}\left(P_{d c}+P_{a c}\right) \operatorname{Cos}\left(\omega_{t h} \tau_{e x t}\right)}{\frac{\Gamma \beta_{s p}}{\tau_{e}}+\frac{G\left(P_{d c}+P_{a c}\right)}{\sqrt{1+\left(P_{d c}+P_{a c}\right) / P_{s}}}}
$$

Let $\Phi=\frac{1}{\sqrt{1+\left(P_{d c}+P_{a c}\right) / P_{s}}}$ and $\Psi=\frac{1}{c o+G(P d c+P a c) \Phi}$, where $c o=\frac{\Gamma \beta_{s p}}{\tau_{e}}$. Expanding $\Phi$ and $\Psi$ up to the third order using Taylor series such that: 


$$
\Phi=M 0+M 1 P_{a c}+M 2 P_{a c}^{2}+M 3 P_{a c}^{3}
$$

and

$$
\Psi=D 0+D 1 P_{a c}+D 2 P_{a c}^{2}+D 3 P_{a c}^{3}
$$

The following Taylor series coefficients were extracted using Mathematica.

$$
\begin{aligned}
& M 0=\frac{1}{\sqrt{1+P_{d c} / P_{s}}} \\
& M 1=-\frac{1}{2\left(1+\left(P_{d c} / P_{s}\right)^{3 / 2} P_{s}\right.} \\
& M 2=\frac{1}{8\left(1+P_{d c} / P_{s}\right)^{5 / 2} P_{s}^{2}} \\
& M 3=-\frac{1}{16\left(1+P_{d c} / P_{s}\right)^{7 / 2} P_{s}^{3}} \\
& D 0=\frac{1}{c o+G M 0 P_{d c}} \\
& D 1=-\frac{G M 0+G M 1 P_{d c}}{\left(c o+G M 0 P_{d c}\right)^{2}} \\
& D 2=\frac{1}{2}\left(\frac{2\left(G M 0+G M 1 P_{d c}\right)^{2}}{\left(c o+G M 0 P_{d c}\right)^{3}}-\frac{2 G M 1+2 G M 2 P_{d c}}{\left(c o+G M 0 P_{d c}\right)^{2}}\right) \\
& D 3=\frac{1}{6}\left(-\frac{6\left(G M 0+G M 1 P_{d c}\right)^{3}}{\left(c o+G M 0 P_{d c}\right)^{4}}+\frac{6\left(G M 0+G M 1 P_{d c}\right)\left(2 G M 1+2 G M 2 P_{d c}\right)}{\left(c o+G M 0 P_{d c}\right)^{3}}\right. \\
& \left.-\frac{6 G M 2+6 G M 3 P_{d c}}{\left(c o+G M 0 P_{d c}\right)^{2}}\right)
\end{aligned}
$$


Substitution of (7.2.5) and (7.2.6) into (7.2.4) gives the carrier density and its derivative as,

$$
\begin{aligned}
\left(C_{d c}+C_{a c}\right)= & Z 0+Z 1 P_{a c}+Z 2 P_{a c}^{\prime}+Z 3 P_{a c}^{2}+Z 4 P_{a c} P_{a c}^{\prime}+Z 5 P_{a c}^{3}+Z 6 P_{a c}^{2} P_{a c}^{\prime} \\
& +Z 7 P_{a c}^{3} P_{a c}^{\prime} \\
C_{a c}^{\prime}= & Z 1 P_{a c}^{\prime}+Z 2 P_{a c}^{\prime \prime}+2 Z 3 P_{a c} P_{a c}^{\prime}+Z 4\left(P_{a c}^{\prime}\right)^{2}+Z 4 P_{a c} P_{a c}^{\prime \prime}+3 Z 5 P_{a c}^{2} P_{a c}^{\prime} \\
& +2 Z 6 P_{a c}\left(P_{a c}^{\prime}\right)^{2}+Z 6 P_{a c}^{2} P_{a c}^{\prime \prime}+3 Z 7 P_{a c}^{2}\left(P_{a c}^{\prime}\right)^{2}+Z 7 P_{a c}^{3} P_{a c}^{\prime \prime}
\end{aligned}
$$

where,

$$
\begin{array}{rl} 
& Z 0=C_{t r} D 0 G M 0 P_{d c}+\frac{D 0 P_{d c}}{\tau_{p h}}-\frac{2 D 0 \kappa_{e x t} P_{d c} \sin \left(\omega_{t h} \tau_{e x t}\right)}{\tau_{l}} \\
Z 1= & C_{t r} D 0 G M 0+C_{t r} D 1 G M 0 P_{d c}+C_{t r} D 0 G M 1 P_{d c}+\frac{D 0}{\tau_{p h}} \\
& +\frac{D 1 P_{d c}}{\tau_{p h}}-\frac{2 D 0 \kappa_{e x t} \sin \left(\omega_{t h} \tau_{e x t}\right)}{\tau_{l}}-\frac{2 D 1 \kappa_{e x t} P_{d c} \sin \left(\omega_{t h} \tau_{e x t}\right)}{\tau_{l}} \\
Z 3= & C_{t r} D 1 G M 0+C_{t r} D 0 G M 1+C_{t r} D 2 G M 0 P_{d c} \\
& +C_{t r} D 1 G M 1 P_{d c}+C_{t r} D 0 G M 2 P_{d c}+\frac{D 1}{\tau_{p h}} \\
& +\frac{D 2 P_{d c}}{\tau_{p h}}-\frac{2 D 1 \kappa_{e x t} \sin \left(\omega_{t h} \tau_{e x t}\right)}{\tau_{l}}-\frac{2 D 2 \kappa_{e x t} P_{d c} \sin \left(\omega_{t h} \tau_{e x t}\right)}{\tau_{l}} \\
Z & Z 4=D 1
\end{array}
$$




$$
\begin{gathered}
Z 5=C_{t r} D 2 G M 0+C_{t r} D 1 G M 1+C_{t r} D 0 G M 2+C_{t r} D 3 G M 0 P_{d c} \\
+C_{t r} D 2 G M 1 P_{d c}+C_{t r} D 1 G M 2 P_{d c}+C_{t r} D 0 G M 3 P_{d c} \\
+\frac{D 2}{\tau_{p h}}+\frac{D 3 P_{d c}}{\tau_{p h}}-\frac{2 D 2 \kappa_{e x t} \sin \left(\omega_{t h} \tau_{e x t}\right)}{\tau_{l}}-\frac{2 D 3 \kappa_{e x t} P_{d c} \sin \left(\omega_{t h} \tau_{e x t}\right)}{\tau_{l}} \\
Z 6=D 2
\end{gathered}
$$

$$
Z 7=D 3
$$

Substitution of (7.2.5) to (7.2.24) into (7.2.2) gives the modulation current as,

$$
I_{a c}=-I_{d c}+q V_{c}\left[C_{a c}^{\prime}+\frac{C_{a c}+C_{d c}}{\tau_{e}}+g_{m}\left(\left(C_{a c}+C_{d c}\right)-C_{t r}\right) \Phi\left(P_{a c}+P_{d c}\right)\right]
$$

Where $g_{m}=G / \Gamma$. Substitution of (7.2.5), (7.2.15), and (7.2.16) into (7.2.25) and considering terms up to the third order gives,

$$
\begin{aligned}
I_{a c}= & K \\
& +\left\{m_{0} P_{a c}+m_{1} P_{a c}^{\prime}+m_{2} P_{a c}^{\prime \prime}\right\} \\
& +\left\{m_{3} P_{a c}^{2}+m_{4} P_{a c} P_{a c}^{\prime}+m_{5}\left(P_{a c}^{\prime}\right)^{2}+m_{5} P_{a c} P_{a c}^{\prime \prime}\right\} \\
& +\left\{m_{6} P_{a c}^{3}+m_{7} P_{a c}^{2} P_{a c}^{\prime}+2 m_{8} P_{a c}\left(P_{a c}^{\prime}\right)^{2}+m_{8} P_{a c}^{2} P_{a c}^{\prime \prime}\right\}
\end{aligned}
$$

The laser's inverse model is represented by the input-output relation given by (7.2.26). The first row is a constant. The second, third and fourth rows will be used to extract the first, second, and third order Volterra transfer functions respectively. The constants $m_{0}$ to $m_{8}$ have been calculated using Mathematica.

$$
\begin{aligned}
m_{0}= & -C_{t r} g_{m} M 0 q V_{c}-C_{t r} g_{m} M 1 P_{d c} q V_{c}+g_{m} M 0 q V_{c} Z 0 \\
& +g_{m} M 1 P_{d c} q V_{c} Z 0+g_{m} M 0 P_{d c} q V_{c} Z 1+\frac{q V_{c} Z 1}{\tau_{e}}
\end{aligned}
$$




$$
m_{1}=q V_{c} Z 1+g_{m} M 0 P_{d c} q V_{c} Z 2+\frac{q V_{c} Z 2}{\tau_{e}}
$$

$$
m_{2}=q V_{c} Z 2
$$

$$
\begin{aligned}
m_{3}= & -C_{t r} g_{m} M 1 q V_{c}-C_{t r} g_{m} M 2 P_{d c} q V_{c}+g_{m} M 1 q V_{c} Z 0+g_{m} M 2 P_{d c} q V_{c} Z 0 \\
& +g_{m} M 0 q V_{c} Z 1+g_{m} M 1 P_{d c} q V_{c} Z 1+g_{m} M 0 P_{d c} q V_{c} Z 3+\frac{q V_{c} Z 3}{\tau_{e}}
\end{aligned}
$$

$$
m_{4}=g_{m} M 0 q V_{c} Z 2+g_{m} M 1 P_{d c} q V_{c} Z 2+2 q V_{c} Z 3+g_{m} M 0 P_{d c} q V_{c} Z 4+\frac{q V_{c} Z 4}{\tau_{e}}
$$

$$
m_{5}=q V_{c} Z 4
$$

$$
\begin{aligned}
m_{6}= & -C_{t r} g_{m} M 2 q V_{c}-C_{t r} g_{m} M 3 P_{d c} q V_{c}+g_{m} M 2 q V_{c} Z 0+g_{m} M 3 P_{d c} q V_{c} Z 0+g_{m} M 1 q V_{c} Z 1 \\
& +g_{m} M 2 P_{d c} q V_{c} Z 1+g_{m} M 0 q V_{c} Z 3+g_{m} M 1 P_{d c} q V_{c} Z 3+g_{m} M 0 P_{d c} q V_{c} Z 5+\frac{q V_{c} Z 5}{\tau_{e}}
\end{aligned}
$$

$$
\begin{aligned}
m_{7}= & g_{m} M 1 q V_{c} Z 2+g_{m} M 2 P_{d c} q V_{c} Z 2+g_{m} M 0 q V_{c} Z 4+g_{m} M 1 P_{d c} q V_{c} Z 4 \\
& +3 q V_{c} Z 5+g_{m} M 0 P_{d c} q V_{c} Z 6+\frac{q V_{c} Z 6}{\tau_{e}}
\end{aligned}
$$

$$
m_{8}=q V_{c} Z 6
$$




\subsubsection{Volterra Series}

The Volterra series technique is used to calculate and analyze the distortion terms in non-linear electronic elements. The inverse semiconductor laser model is obtained by considering the laser as a non-linear system with an input of $P(t)$ and an output of $I(t)$. Using Volterra series [81], the system output is given by:

$$
\begin{aligned}
I(t)= & \int_{-\infty}^{+\infty} g_{1}\left(\tau_{1}\right) P\left(t-\tau_{1}\right) d \tau_{1}+\int_{-\infty}^{\infty} \int_{-\infty}^{\infty} g_{2}\left(\tau_{1}, \tau_{1}\right) P\left(t-\tau_{1}\right) P\left(t-\tau_{2}\right) d \tau_{1} d \tau_{2} \\
& +\int_{-\infty}^{+\infty} \int_{-\infty}^{+\infty} \int_{-\infty}^{+\infty} g_{3}\left(\tau_{1}, \tau_{2}, \tau_{3}\right) P\left(t-\tau_{1}\right) P\left(t-\tau_{2}\right) P\left(t-\tau_{3}\right) d \tau_{1} d \tau_{2} d \tau_{3}+\ldots \\
& +\int_{-\infty}^{+\infty} \int_{-\infty}^{+\infty} \ldots \int_{-\infty}^{+\infty} g_{n}\left(\tau_{1}, \tau_{2}, \ldots, \tau_{n}\right) P\left(t-\tau_{1}\right) P\left(t-\tau_{2}\right) \ldots P\left(t-\tau_{n}\right) d \tau_{1} d \tau_{2} \ldots d \tau_{n}+\ldots
\end{aligned}
$$

where $g_{n}\left(\tau_{1}, \tau_{2}, \ldots, \tau_{n}\right)=0$ for any $\tau_{m}<0, m=1,2, \ldots, n, n=1,2, \ldots$. The series in the previous equation is called a Volterra series and the functions $g_{n}\left(\tau_{1}, \tau_{2}, \ldots, \tau_{n}\right)$ are called the Volterra kernels of the system.

The above equation can be also expressed as,

$$
I(t)=G_{1}[(P(t))]+G_{2}[(P(t))]+G_{3}[(P(t))]+\ldots+G_{n}[(P(t))]+\ldots
$$

where $G_{n}$, is the $n^{\text {th }}$-order Volterra operator and is given by the following integral,

$$
G_{n}[(P(t))]=\int_{-\infty}^{\infty} \ldots \int_{-\infty}^{\infty} g_{n}\left(\tau_{1}, \ldots, \tau_{n}\right) P\left(t-\tau_{1}\right) \ldots P\left(t-\tau_{n}\right) d \tau_{1} \ldots d \tau_{n}
$$

\subsubsection{Extraction of Volterra Transfer Functions}

\section{Harmonic-input method}

The harmonic-input method is used to determine the kernels $G_{k}$ in the frequency domain. Considering the equation describing $I_{a c}$ as a function of $P_{a c}$ given by (7.2.26). When $k$ 
input tones (at the frequencies $\omega_{1}, \ldots, \omega_{k}$ ) are applied to the laser model, they have the following form,

$$
P_{a c}=P_{d c}\left(e^{j \omega_{1} t}+e^{j \omega_{2} t}+\ldots+e^{j \omega_{k} t}\right)
$$

When one tone input, $P_{a c}=P_{d c} e^{j \omega_{1} t}$, is applied to the system described by (7.2.26), the first order Volterra transfer function is the coefficient of $e^{j \omega_{1} t}$ and is given by,

$$
G_{1}\left(\omega_{1}\right)=\left(m_{0}+j m_{1} \omega_{1}-m_{2} \omega_{1}^{2}\right) P_{d c}
$$

The second order Volterra transfer function is obtained if two tone inputs, $P_{a c}=$ $P_{d c} e^{j \omega_{1} t}+P_{d c} e^{j \omega_{2} t}$, are applied to (7.2.26). The coefficient of $e^{j\left(\omega_{1}+\omega_{2}\right) t}$ represents the second order Volterra transfer function and is given by,

$$
G_{2}\left(\omega_{1}, \omega_{2}\right)=\left(2 m_{3}+j m_{4}\left(\omega_{1}+\omega_{2}\right)-m_{5}\left(\omega_{1}+\omega_{2}\right)^{2}\right) P_{d c}^{2}
$$

If three tone inputs, $P_{a c}=P_{d c} e^{j \omega_{1} t}+P_{d c} e^{j \omega_{2} t}+P_{d c} e^{j \omega_{3} t}$, are applied to (7.2.26), the third order Volterra transfer function is the coefficient of $e^{j\left(\omega_{1}+\omega_{2}+\omega_{3}\right) t}$ and is given by,

$$
G_{3}\left(\omega_{1}, \omega_{2}, \omega_{3}\right)=\left(6 m_{6}+2 j m_{7}\left(\omega_{1}+\omega_{2}+\omega_{3}\right)-2 m_{8}\left(\omega_{1}+\omega_{2}+\omega_{3}\right)^{2}\right) P_{d c}^{3}
$$

Equations (7.2.40) to (7.2.42) represent the laser inverse model frequency domain Volterra transfer functions.

\section{Direct expansion method}

The direct expansion method is used to derive the time domain laser inverse model from the frequency domain model. Equations (7.2.40) to (7.2.42) can be represented by their Laplace transforms easily by replacing each $j \omega$ by $s$ as follows,

$$
G_{1}\left(s_{1}\right)=\left(m_{0}+m_{1} s_{1}+m_{2} s_{1}^{2}\right) P_{d c}
$$




$$
\begin{gathered}
G_{2}\left(s_{1}, s_{2}\right)=\left(2 m_{3}+m_{4}\left(s_{1}+s_{2}\right)+m_{5}\left(s_{1}+s_{2}\right)^{2}\right) P_{d c}^{2} \\
G_{3}\left(s_{1}, s_{2}, s_{3}\right)=\left(6 m_{6}+2 m_{7}\left(s_{1}+s_{2}+s_{3}\right)+2 m_{8}\left(s_{1}+s_{2}+s_{3}\right)^{2}\right) P_{d c}^{3}
\end{gathered}
$$

Applying the inverse Laplace transformation to (7.2.43) to (7.2.45) to get their equivalents in the time domain as,

$$
\begin{gathered}
G_{1}[P(t)]=m_{0} P(t)+m_{1} \frac{d P(t)}{d t}+m_{2} \frac{d^{2} P(t)}{d t^{2}} \\
G_{2}[P(t)]=2 m_{3} P^{2}(t)+m_{4} \frac{d\left(P^{2}(t)\right)}{d t}+m_{5} \frac{d^{2}\left(P^{2}(t)\right)}{d t^{2}} \\
G_{3}[P(t)]=6 m_{6} P^{3}(t)+2 m_{7} \frac{d\left(P^{3}(t)\right)}{d t}+2 m_{8} \frac{d^{2}\left(P^{3}(t)\right)}{d t^{2}}
\end{gathered}
$$

Equations (7.2.46) to (7.2.48) represent the laser inverse model in time domain. The complete third order time domain Volterra system of the pre-distorter is illustrated in Figure 7.1 on the next page.

An amplifier with gain $m_{0}$ was used to represent the first term of (7.2.46), the second term was represented by an amplifier with gain $m_{1}$ and a differentiator, and the third term was represented by an an amplifier with gain $m_{2}$ and two differentiators.

Equation (7.2.47) was represented by an amplifier with gain $2 m_{3}$ for the first term, an amplifier with gain $m_{4}$ and a differentiator for the second term, and an amplifier with gain $m_{5}$ and two differentiators for the third term.

First term of (7.2.48) was represented by an amplifier with gain $6 m_{6}$, second term was represented by an amplifier with gain $2 m_{7}$ and a differentiator, and the third term was represented by an amplifier with gain $2 m_{8}$ and two differentiators.

The constants $m_{0}$ to $m_{8}$ are functions of the laser parameters. The laser parameters are sensitive to thermal effects and aging, which requires the pre-distorter coefficients to 


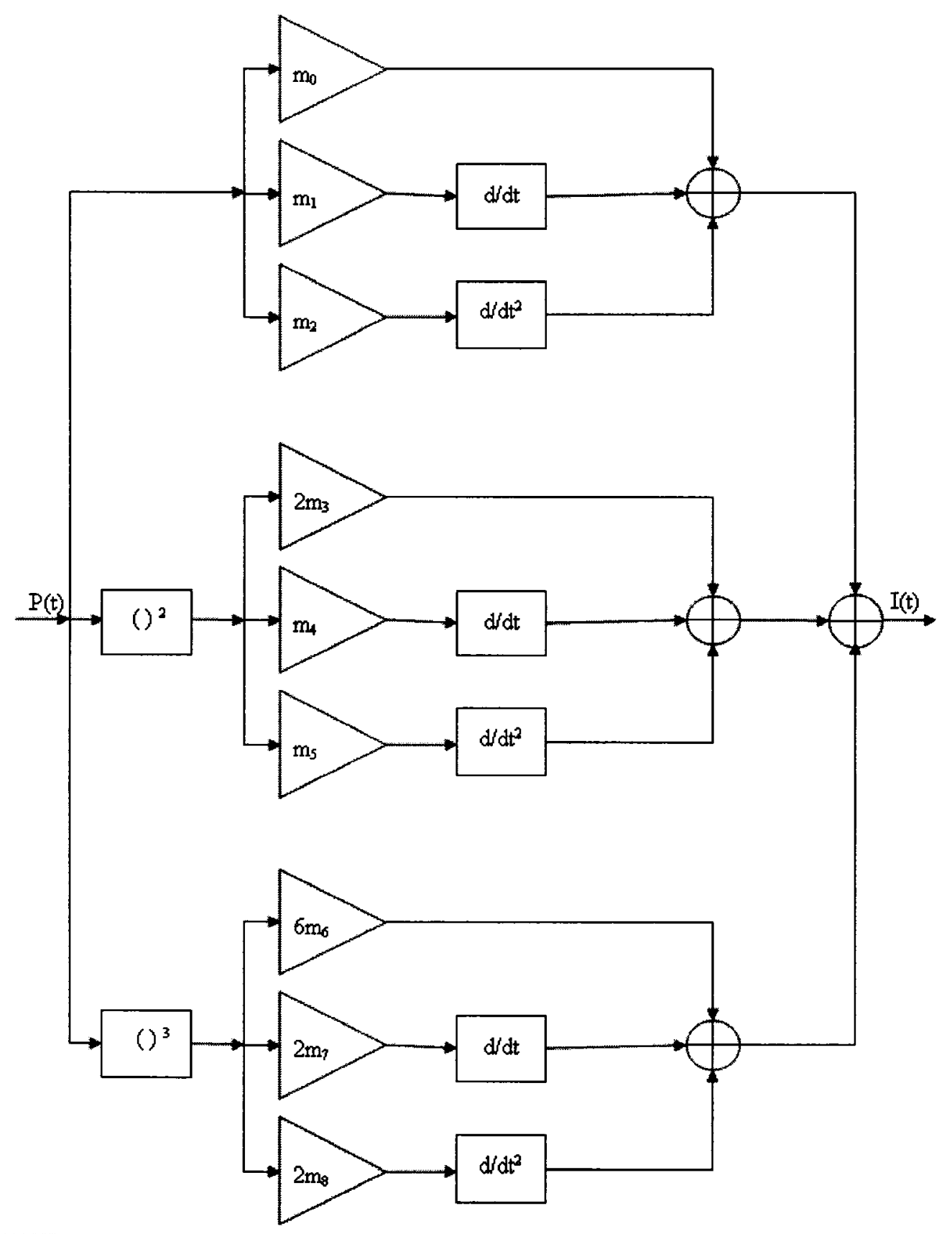

Figure 7.1: Block diagram of the laser inverse model. 
be adjusted accordingly in order to compensate for these changes in the laser parameters. This addresses the need for an efficient way to sense the laser output and give feedback to the pre-distorter with these changes to modify its coefficients. Adaptive pre-distorters can monitor the lasers output and update the pre-distorter coefficients and compensate for any of the lasers' time-varying changes.

\subsection{Inverse Laser Model Including Feedback Imple- mentation}

The above pre-distorter model was implemented in ADS using three branches, each branch containing two dual-port SDDs representing equations (7.2.46), (7.2.47), and (7.2.48) respectively. Figure 7.2 on the following page shows the proposed pre-distorter implementation in ADS.

The setup shown in Figure 7.3 on page 126 is used to predict the ability of the proposed pre-distorter to reduce the harmonic distortions of the laser, the implemented pre-distorter was cascaded to the laser model and compared to the same laser model without a predistorter. To examine the improvement of the second and the third harmonic distortions due to the existence of the pre-distorter, the frequency of a single tone input was swept up to $4 \mathrm{GHz}$, the second and the third harmonic distortions with and without using the pre-distorter were simulated for 0.10 and 0.25 modulation index.

Figure 7.4 on page 127 shows the simulated second harmonic distortion with and without using the pre-distorter for 0.10 and 0.25 modulation index. The results showed that an improvement up to $30 \mathrm{~dB}$ (for 0.25 modulation index) and $45 \mathrm{~dB}$ (for 0.10 modulation index) over bandwidth around $3 \mathrm{GHz}$, between $1 \mathrm{GHz}$ and $4 \mathrm{GHz}$, can be achieved using the proposed pre-distorter.

Figure 7.5 on page 127 shows the simulated third harmonic distortion with and without using the pre-distorter for for 0.10 and 0.25 modulation index. The results showed that an improvement up to $40 \mathrm{~dB}$ (for 0.25 modulation index) and $55 \mathrm{~dB}$ (for 0.10 modulation index) over bandwidth around $3 \mathrm{GHz}$, from $1 \mathrm{GHz}$ to $4 \mathrm{GHz}$, can be achieved using the proposed pre-distorter. 


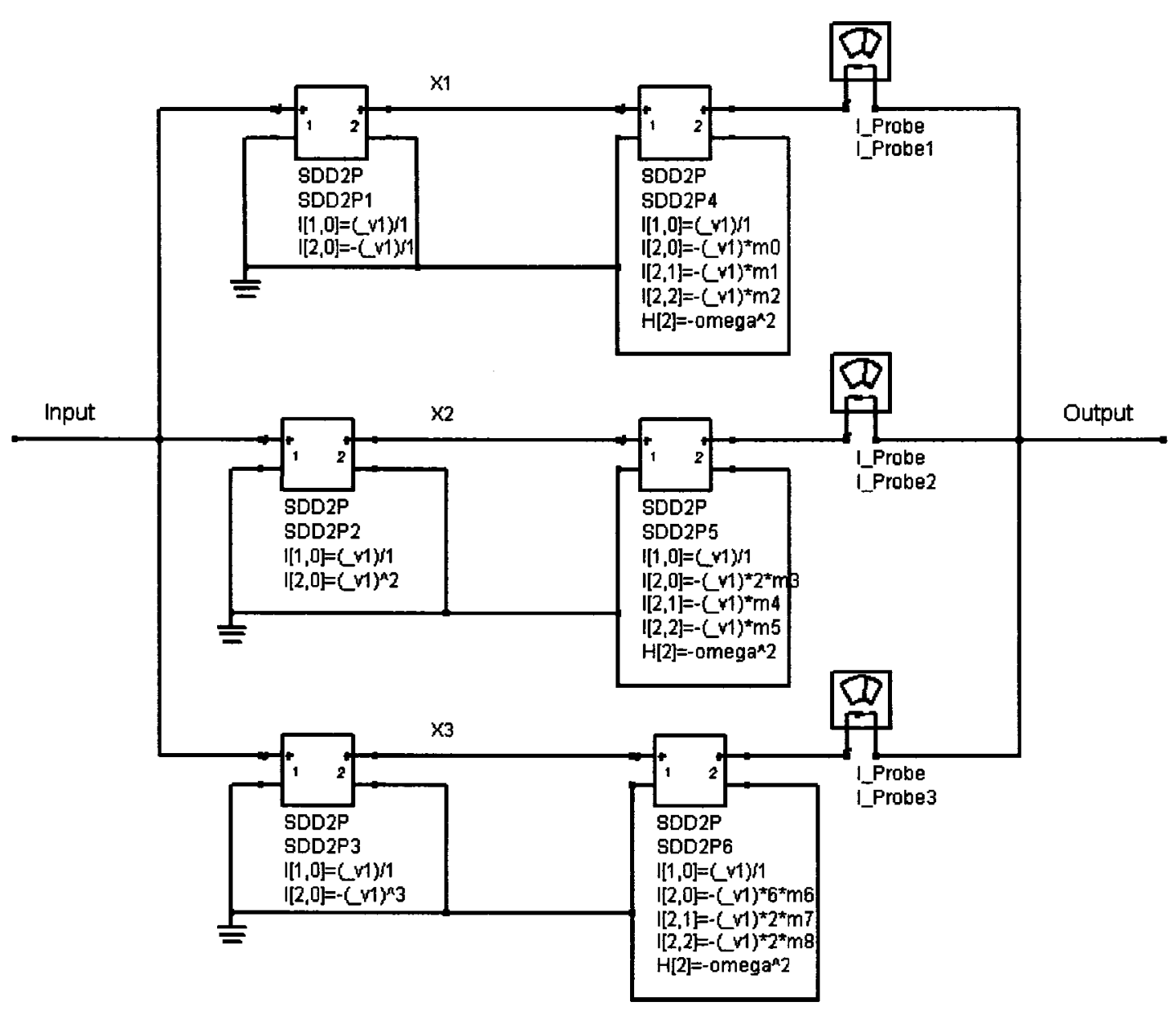

Figure 7.2: ADS implementation of the laser inverse model. 


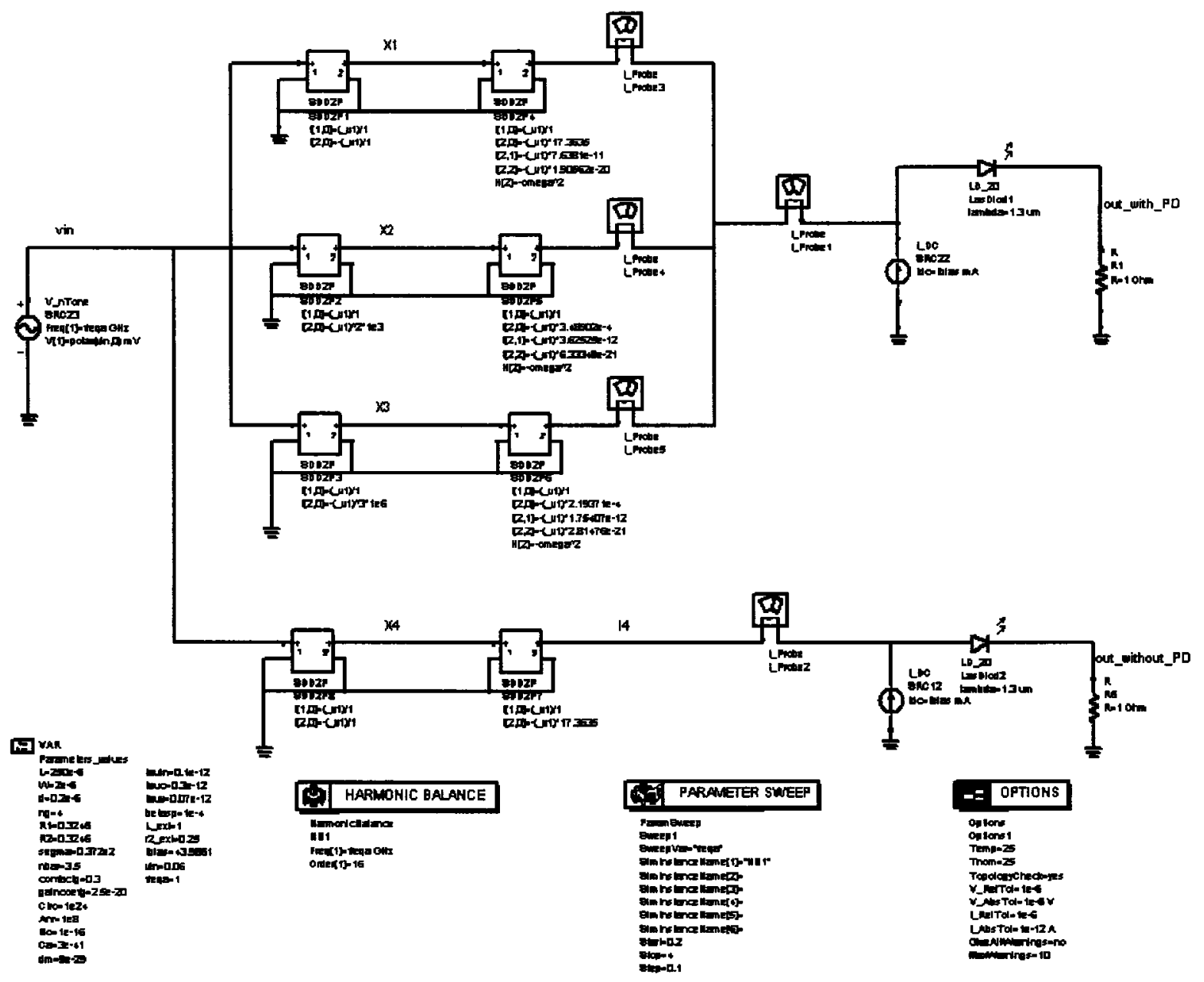

Figure 7.3: ADS setup of the laser inverse model cascaded to the laser model. 


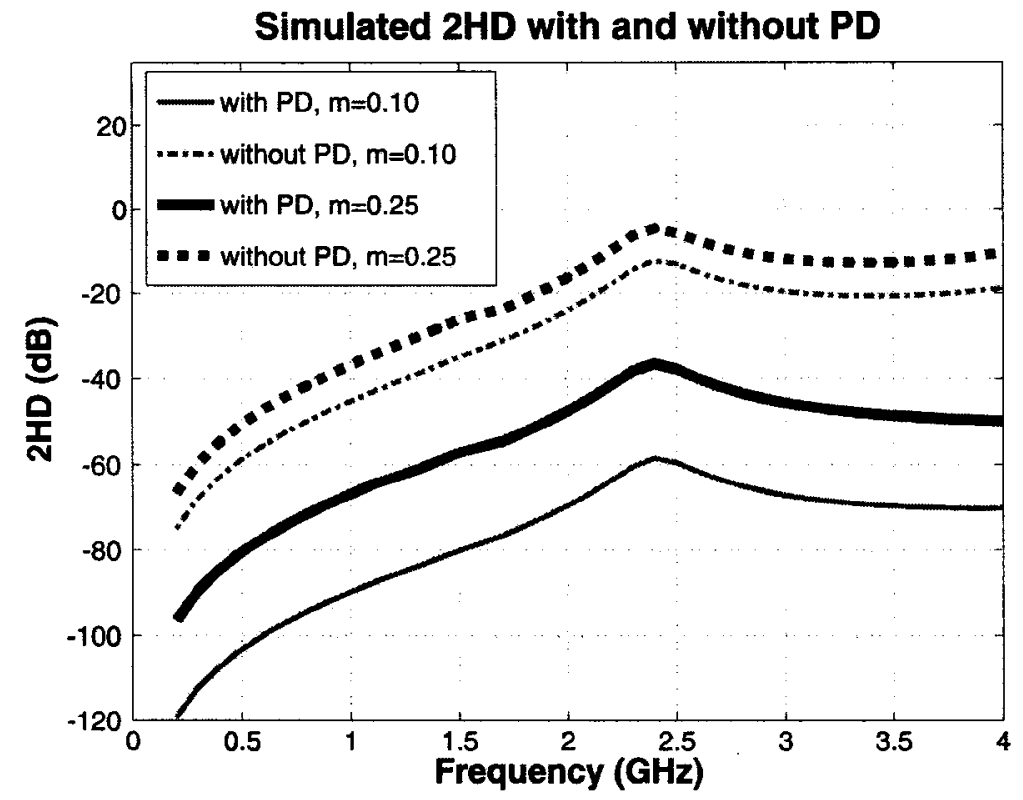

Figure 7.4: Simulated second order harmonic with and without PD for 0.10 modulation index and 0.25 modulation index.

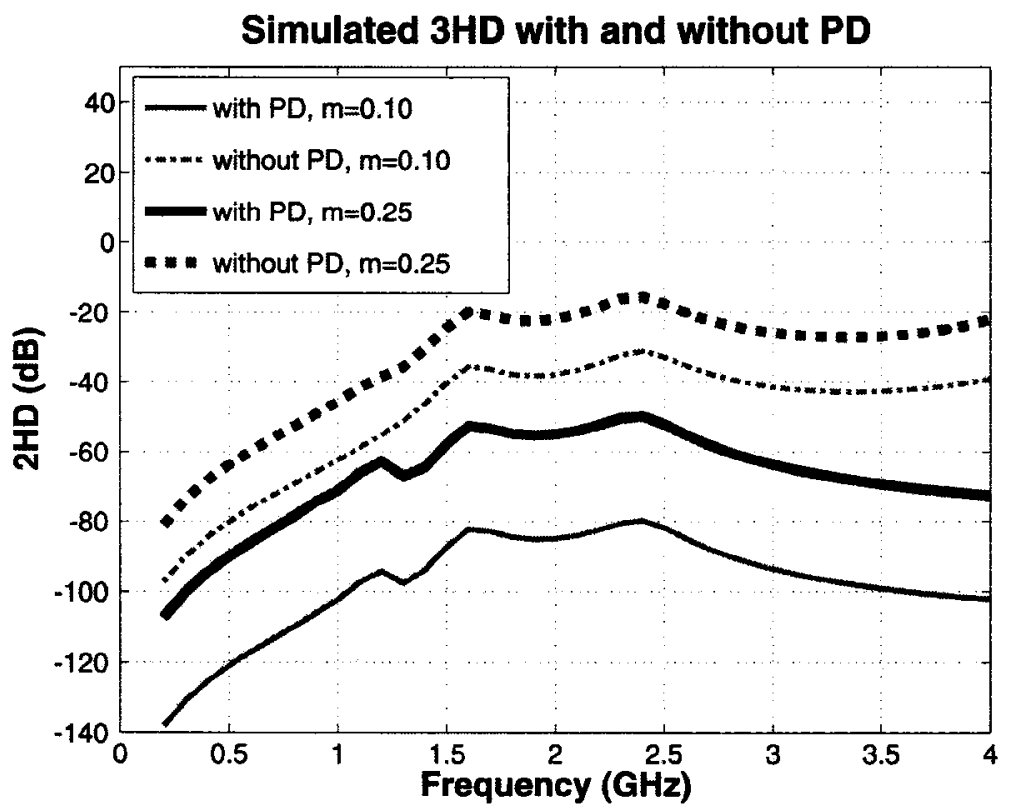

Figure 7.5: Simulated third order harmonic with and without PD for 0.10 modulation index and 0.25 modulation index. 
To simulate the second and third intermodulation distortions (2IMD and 3IMD), the cascaded laser model and the pre-distorter were directly modulated with two tones that are $10 \mathrm{MHz}$ apart and monitoring the generated sum and difference frequencies. These included, the second intermodulation distortion $\left(f_{1}+f_{2}\right)$, the third intermodulation distortions $\left(2 f_{1}+\right.$ $f_{2}, 2 f_{1}-f_{2}, 2 f_{2}+f_{1}$, and $\left.2 f_{2}-f_{1}\right)$.

Figures 7.6 - 7.10 on page 132 show how the proposed pre-distorter decreases the aforementioned intermodulation distortion components.

Figure 7.6 illustrates the simulation results of the second order intermodulation distortion of type $\left(f_{1}+f_{2}\right)$ for modulation index of 0.10 . Results show that the pre-distorter has reduced the second order intermodulation distortion by an average of $50 \mathrm{~dB}$ over a bandwidth of $2 \mathrm{GHz}$, from $2 \mathrm{GHz}$ to $4 \mathrm{GHz}$. A peak was observed around a frequency of $2.4 \mathrm{GHz}$ which may attributed to one of the intermodulation frequencies of $f_{1}$ and $f_{2}$.

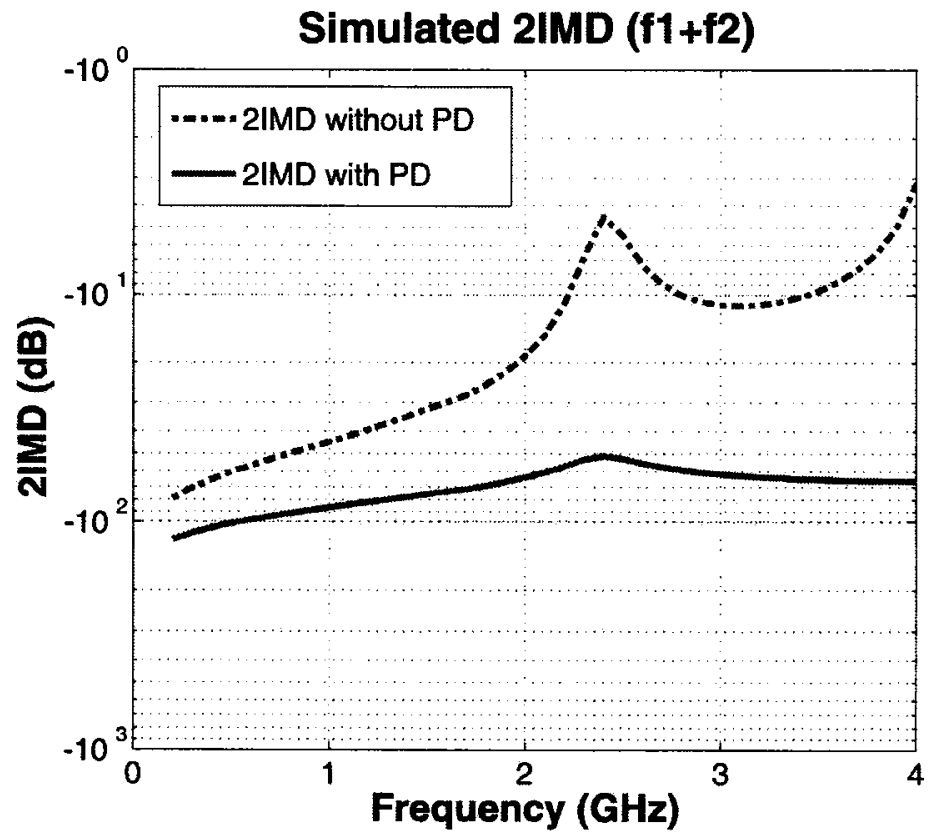

Figure 7.6: Simulated second intermodulation distortion $\left(f_{1}+f_{2}\right)$ with and without PD for 0.10 modulation index.

Figure 7.7 on the following page shows the simulation results of the third order intermodulation distortion $\left(2 f_{1}+f_{2}\right)$ for modulation index of 0.10 . The pre-distorter has 
reduced the third order intermodulation distortion by an average of $40 \mathrm{~dB}$ over bandwidth of $2.5 \mathrm{GHz}$, between $1.5 \mathrm{GHz}$ and $4 \mathrm{GHz}$. Two peaks were observed around frequencies of $1.6 \mathrm{GHz}$ and $2.4 \mathrm{GHz}$, these may also attributed to some of $f_{1}$ and $f_{2}$ intermodulation frequencies.

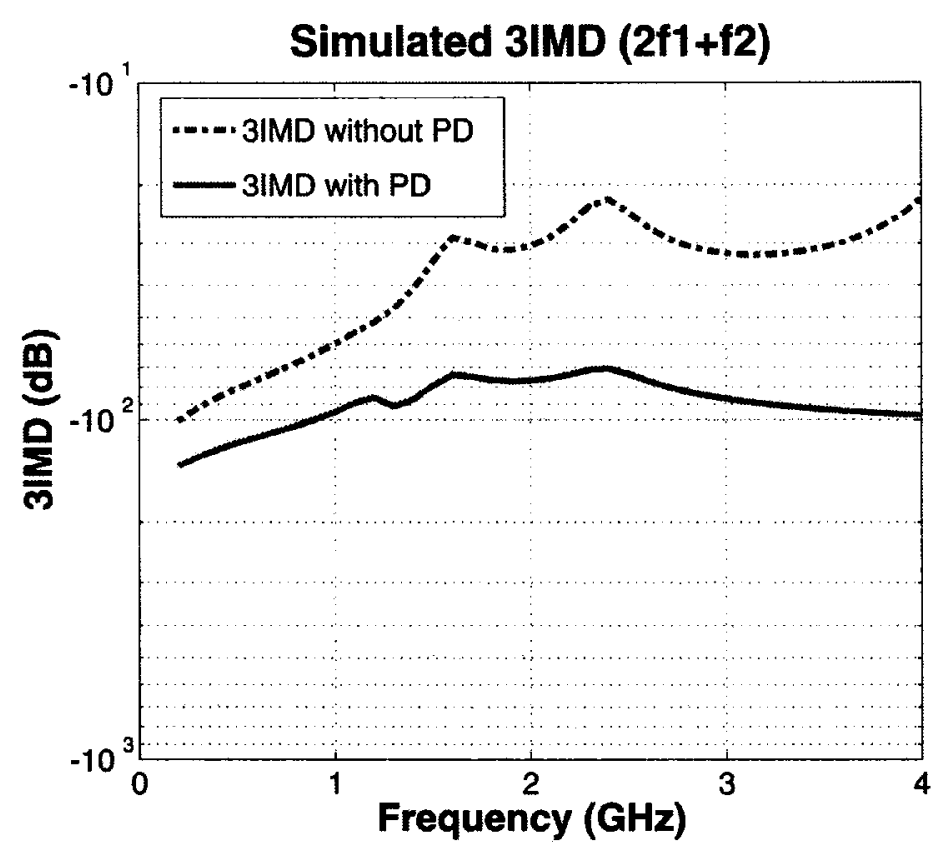

Figure 7.7: Simulated third intermodulation distortion $\left(2 f_{1}+f_{2}\right)$ with and without PD for 0.10 modulation index.

Figure 7.8 on the next page shows the simulation results of the third order intermodulation distortion $\left(2 f_{1}-f_{2}\right)$ for modulation index of 0.10 . An average enhancement of $35 \mathrm{~dB}$ for the 3IMD of type $\left(2 f_{1}-f_{2}\right)$ has been achieved over bandwidth of $2 \mathrm{GHz}$, between $2 \mathrm{GHz}$ and $4 \mathrm{GHz}$.

Figure 7.9 on page 131 illustrates the simulation results of the third order intermodulation distortion $\left(2 f_{2}+f_{1}\right)$ for modulation index of 0.10 . Using the proposed pre-distorter improved the 3IMD of type $\left(2 f_{2}+f_{1}\right)$ by an average value of $40 \mathrm{~dB}$ over $2.5 \mathrm{GHz}$ bandwidth, from $1.5 \mathrm{GHz}$ to $4 \mathrm{GHz}$. The two peaks that perviously observed with $\left(2 f_{1}+f_{2}\right)$ intermodulation distortion type are also observed with $\left(2 f_{1}+f_{2}\right)$ type.

Figure 7.10 on page 132 shows the simulation results of the third order intermodulation 


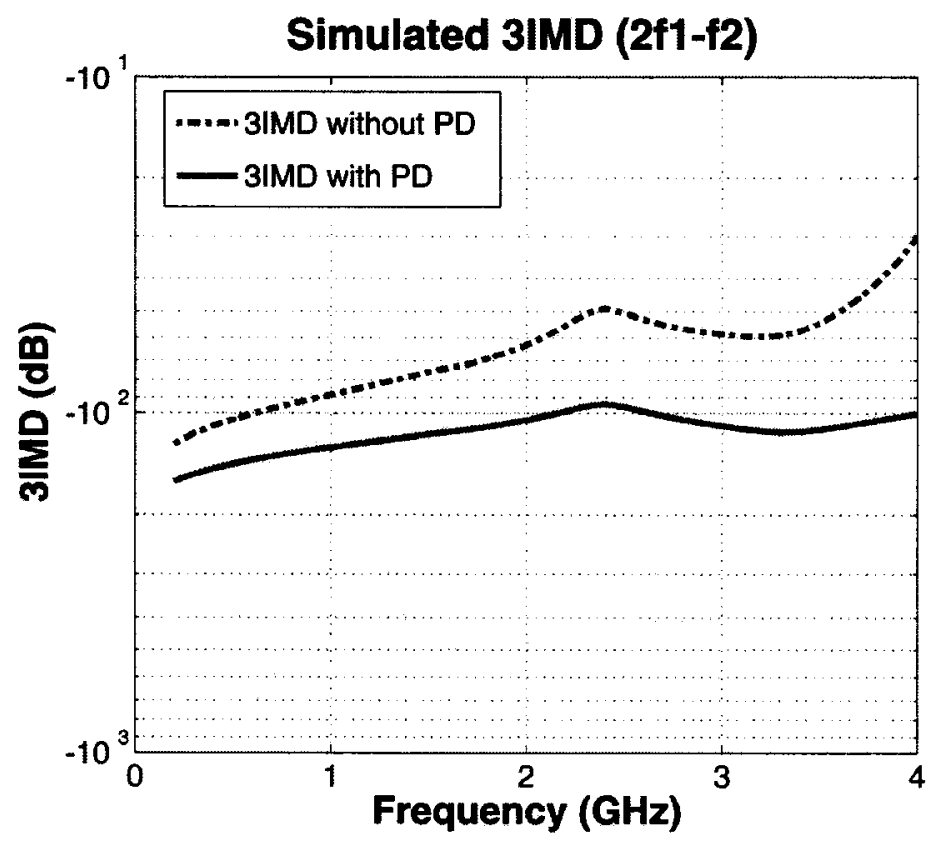

Figure 7.8: Simulated third intermodulation distortion $\left(2 f_{1}-f_{2}\right)$ with and without PD for 0.10 modulation index. 


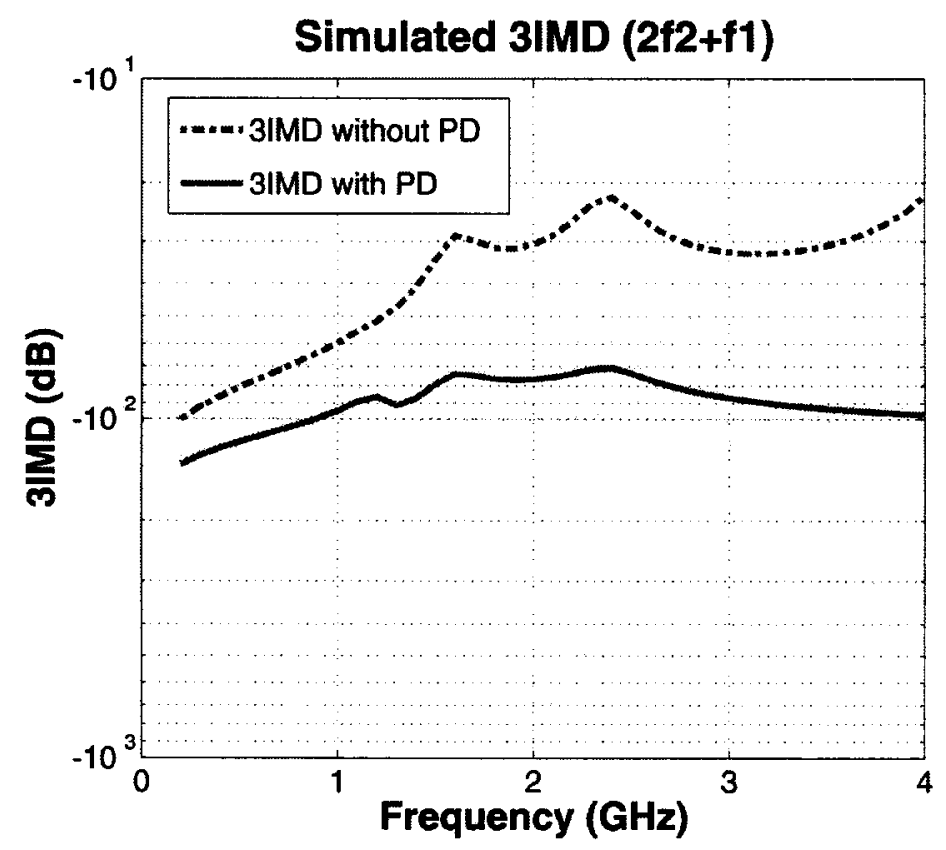

Figure 7.9: Simulated third intermodulation distortion $\left(2 f_{2}+f_{1}\right)$ with and without PD for 0.10 modulation index. 
distortion $\left(2 f_{2}-f_{1}\right)$ for modulation index of 0.10 . As can be seen from the results, the predistorter has reduced the 3IMD of type $\left(2 f_{2}-f_{1}\right)$ by an average of $35 \mathrm{~dB}$ over bandwidth of $2 \mathrm{GHz}$, from $2 \mathrm{GHz}$ to $4 \mathrm{GHz}$.

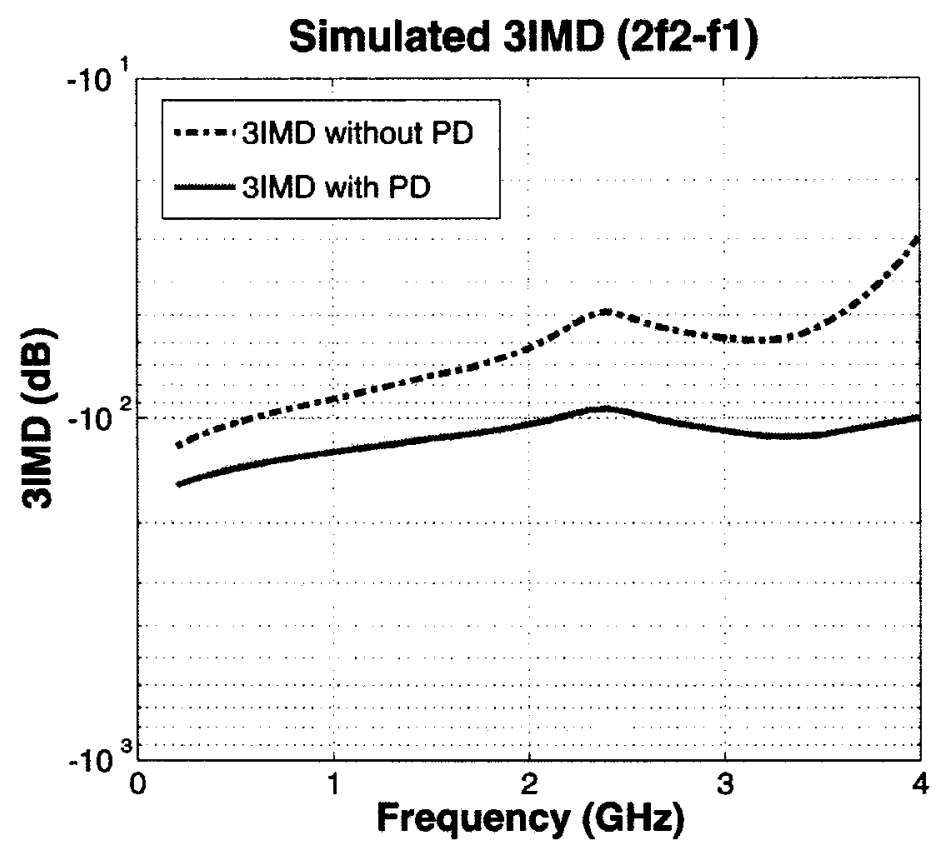

Figure 7.10: Simulated third intermodulation distortion $\left(2 f_{2}-f_{1}\right)$ with and without PD for 0.10 modulation index.

The above results are summarized in Table 7.1.

\subsection{Typical CATV system}

To characterize CATV system linearity, $\mathrm{N}$ signals of equal power level are applied to the system spaced $6 \mathrm{MHz}$ apart [47]. In order to meet CSO and CTB requirements for CATV systems, the laser's second order harmonic distortion and third order intermodulation distortion of types $\left(2 f_{1} \pm f_{2}\right.$ and $\left.2 f_{2} \pm f_{1}\right)$ must be in the vicinity of $-75 \mathrm{dBc}$ and $-100 \mathrm{dBc}$ respectively, for a modulation index of 0.04 [4].

Figure 7.11 on page 134 shows the simulated second harmonic distortion with and without using the pre-distorter for 0.04 modulation index. The results showed that an average improvement of $45 \mathrm{~dB}$ over bandwidth around $3.5 \mathrm{GHz}$, between $0.5 \mathrm{GHz}$ and $4 \mathrm{GHz}$, can 


\begin{tabular}{|c|c|c|}
\hline Distortion Type & Average Improvement [dB] & Modulation Index \\
\hline$\overline{2^{n d} H D}$ & 45 & $\overline{0.10}$ \\
\hline $2^{\text {nd }} H D$ & 30 & 0.25 \\
\hline $3^{r d} H D$ & 55 & 0.10 \\
\hline $3^{r d} H D$ & 40 & $\overline{0.25}$ \\
\hline $2^{n d} I M D$ type $f_{1}+f_{2}$ & 50 & 0.10 \\
\hline $3^{\text {rd }} I M D$ type $2 f_{1}+f_{2}$ & 40 & 0.10 \\
\hline $3^{r d} I M D$ type $2 f_{1}-f_{2}$ & 35 & 0.10 \\
\hline $3^{r d} I M D$ type $2 f_{2}+f_{1}$ & 40 & 0.10 \\
\hline $3^{\text {rd }} I M D$ type $2 f_{2}-f_{1}$ & 35 & 0.10 \\
\hline
\end{tabular}

Table 7.1: Various distortion components improvement due to using the proposed predistorter.

be achieved using the proposed pre-distorter. Figure 7.12 on page 135 shows the simulated third harmonic distortion with and without using the pre-distorter for 0.04 modulation index. The results showed that an average improvement of $50 \mathrm{~dB}$ over bandwidth around $3.5 \mathrm{GHz}$, between $0.5 \mathrm{GHz}$ and $4 \mathrm{GHz}$, can be achieved using the proposed pre-distorter. The second and third order harmonic distortions levels were decreased to values that are lower than $-75 \mathrm{~dB}$ and $-100 \mathrm{~dB}$ respectively.

The cascaded laser model and the pre-distorter were directly modulated with two tones that are $6 \mathrm{MHz}$ apart. Various types of third order intermodulation distortions $\left(2 f_{1} \pm f_{2}\right.$ and $2 f_{2} \pm f_{1}$ ) were simulated.

Figures 7.13 - 7.16 on page 137 show the simulation results of various third order intermodulation distortions for modulation index of 0.04 . The pre-distorter has decreased all types of third order intermodulation distortions to values that are lower than $-100 \mathrm{~dB}$. 


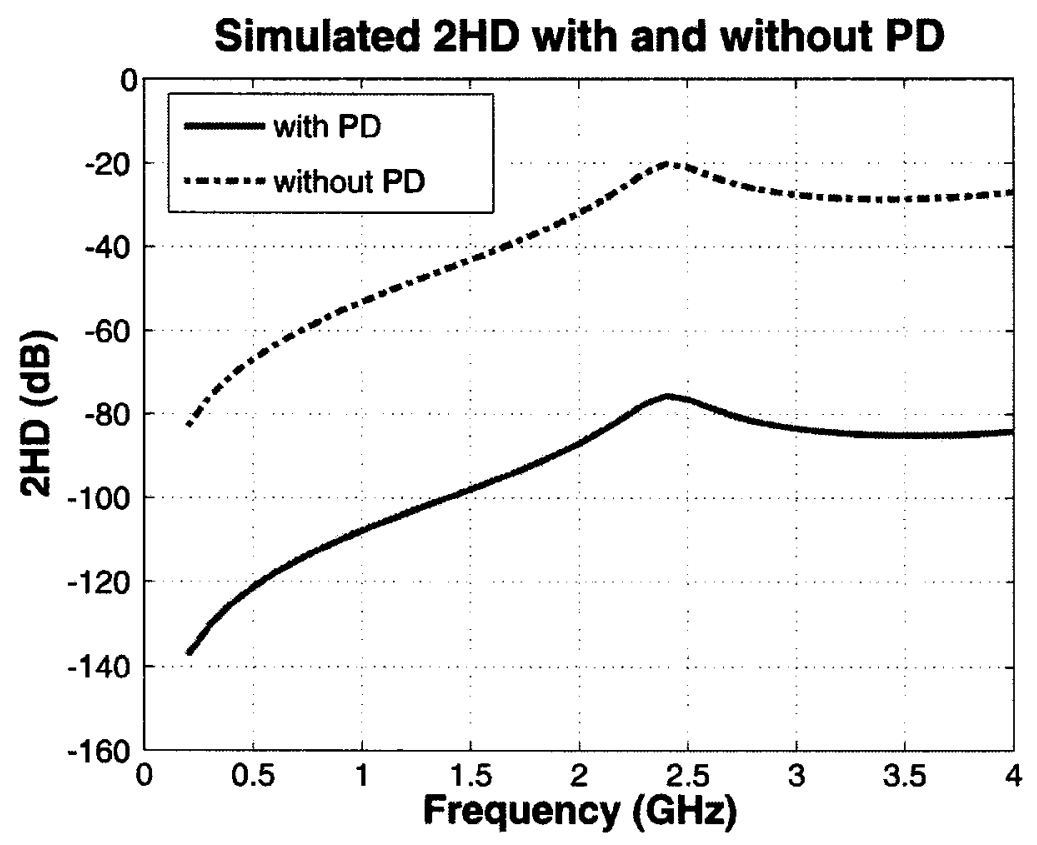

Figure 7.11: Simulated second order harmonic with and without PD for 0.04 modulation index. 


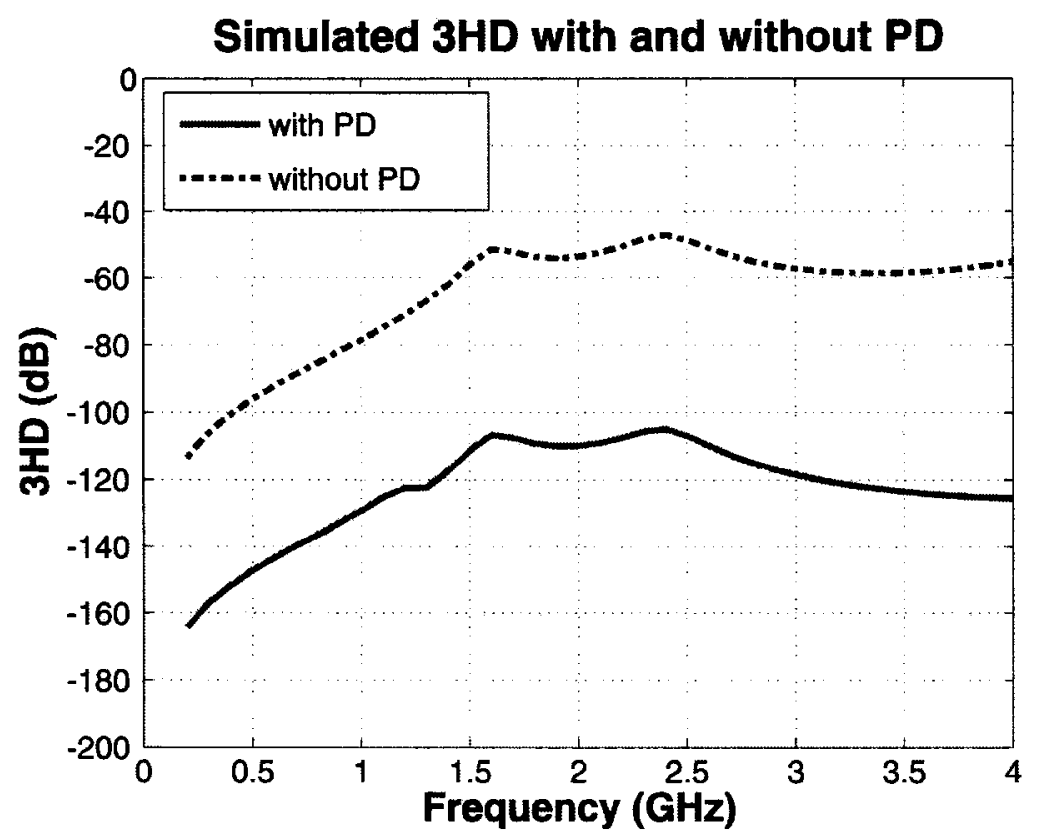

Figure 7.12: Simulated third order harmonic with and without PD for 0.04 modulation index.

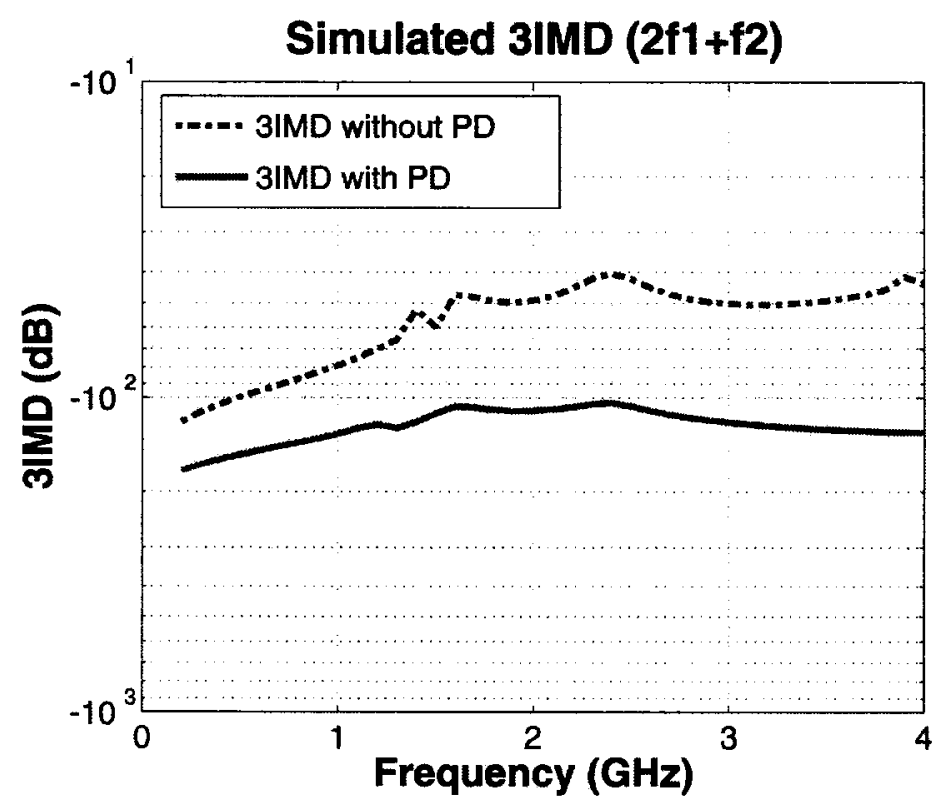

Figure 7.13: Simulated third intermodulation distortion $\left(2 f_{1}+f_{2}\right)$ with and without PD for 0.04 modulation index. 


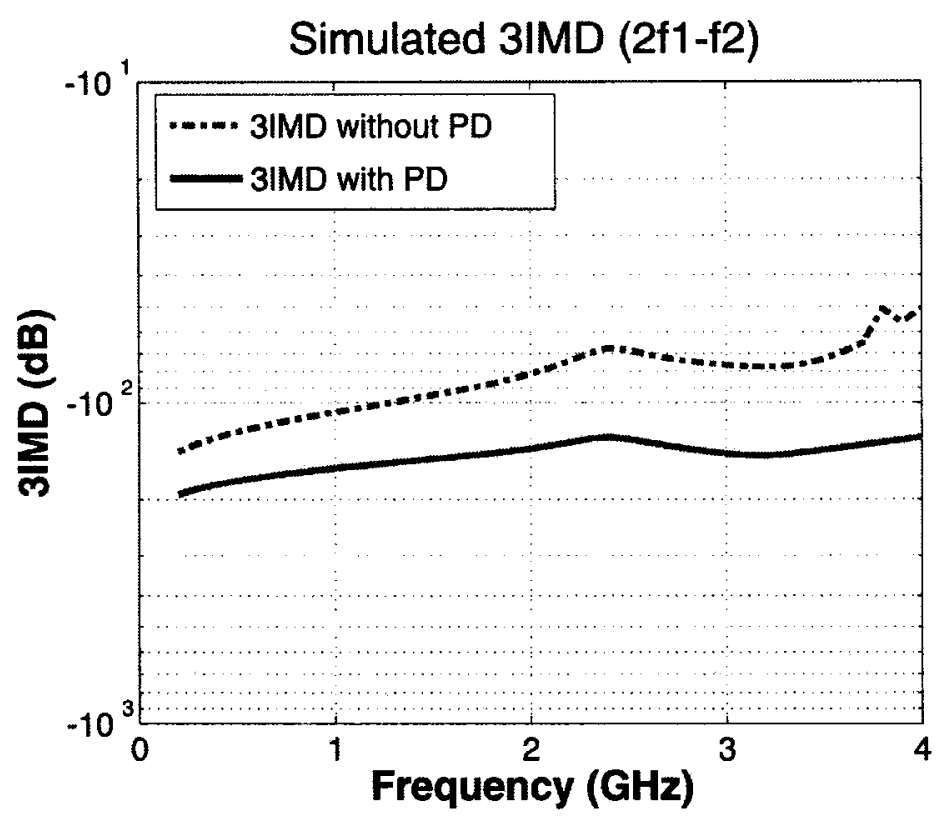

Figure 7.14: Simulated third intermodulation distortion $\left(2 f_{1}-f_{2}\right)$ with and without PD for 0.04 modulation index.

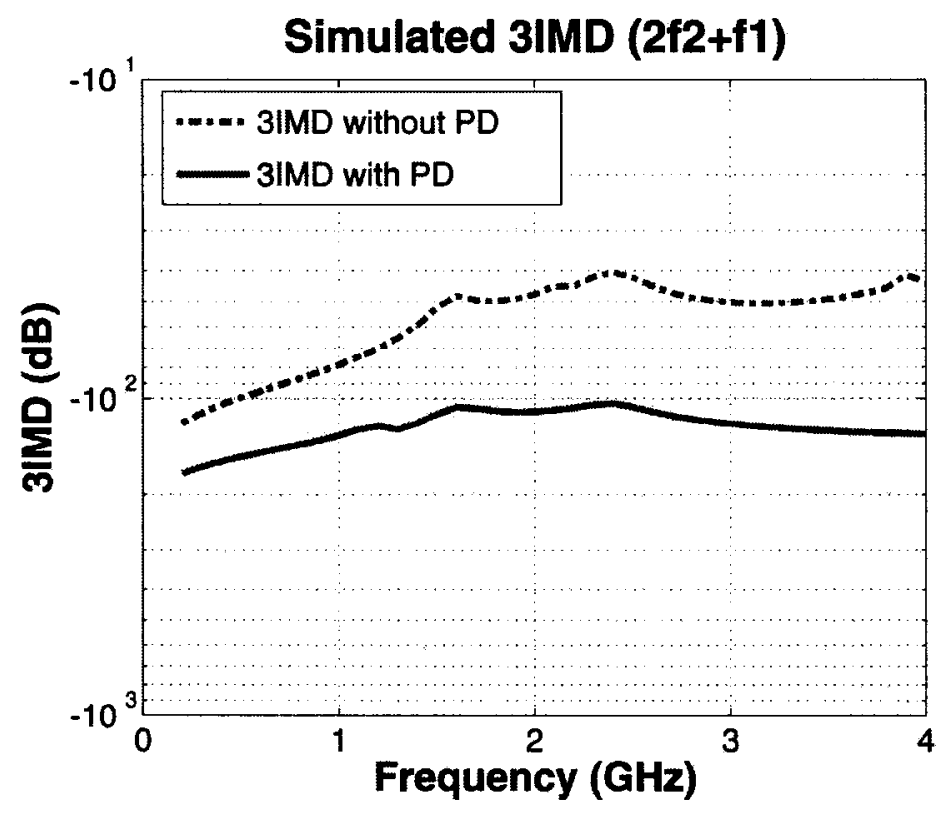

Figure 7.15: Simulated third intermodulation distortion $\left(2 f_{2}+f_{1}\right)$ with and without PD for 0.04 modulation index. 


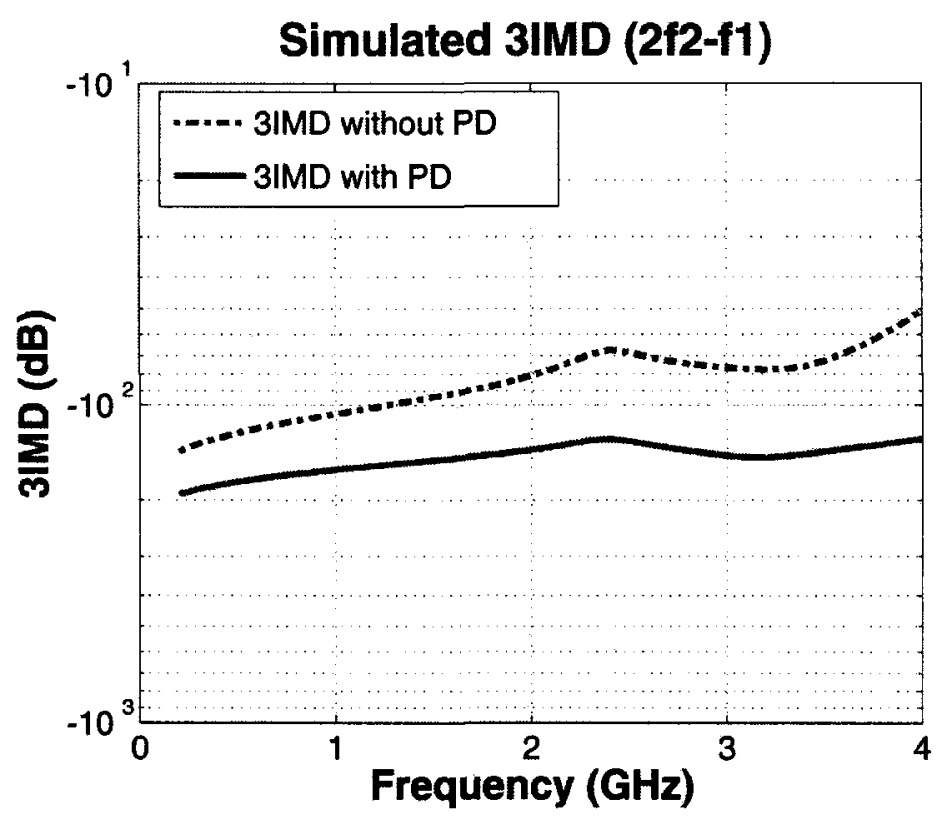

Figure 7.16: Simulated third intermodulation distortion $\left(2 f_{2}-f_{1}\right)$ with and without PD for 0.04 modulation index.

\subsection{Summary}

In this chapter we developed a laser pre-distorter based on the inverse laser model including the feedback effect. Volterra series technique was used to extract the pre-distorter coefficients. A pre-distorter model was implemented in ADS and cascaded to the laser model. Various laser distortion components were simulated both with and without the pre-distorter. Simulation results showed that, at a modulation index of 0.1 , average improvements of $45 \mathrm{~dB}, 55 \mathrm{~dB}$, and $40 \mathrm{~dB}$ were achieved for second order, third order harmonic distortion, and various intermodulation distortions respectively when using the proposed pre-distorter. At modulation index of 0.04 , the pre-distorter has decreased the second order harmonic distortion and various types of third order intermodulation distortions to values that are lower than $-75 \mathrm{~dB}$ and $-100 \mathrm{~dB}$ respectively.

The above results show that the proposed pre-distorter significantly improved the DFB laser linearity and make it suitable for the analog CATV applications. 


\section{Chapter 8}

\section{Conclusions and Future Work}

\subsection{Conclusions}

The single mode semiconductor laser rate equations were augmented to include effects of optical back-reflections. These augmented rate equations were incorporated in the implementation of a semiconductor laser model using Agilent Advanced Design System (ADS) [53]. The ADS model was used in simulations to determine the effect of reflected optical power on the laser's DC characteristics, noise, and linearity. The DFB laser diode's threshold level, relaxation oscillation, relative intensity noise, and distortion under optical back-reflections were simulated using various ADS setups. Simulation results were presented along with relevant measurements. Simulation results and experimental measurements were found to be in good agreement.

The operation of an unisolated DFB laser diode in different optical feedback regimes was experimentally investigated. This was achieved using induced tunable back-reflections. The setup involved a fiber optic reflector, a variable attenuator, a polarization controller, and a 3-dB fiber optic beam splitter. Results show that it is desirable to operate a DFB laser diode with optical feedback without the need for incorporating a costly built-in optical isolator. This is achieved by operating the DFB laser in feedback Regime V, at which the DFB laser diode operates on a single longitudinal mode with low RIN and narrow linewidth for all phases of feedback. Moreover, the DFB laser diode will be insensitive to any further external optical back-reflection. This could not only reduce the cost, but may also simplify the construction of the multi function integrated device.

The experimental results were used to determine the conditions needed to force the 
DFB laser diode to operate in feedback Regime V. In other words, we determined how much optical back-reflection is needed to drive the DFB laser diode to operate in feedback Regime V. Based on these findings, a feedback topology that uses a Fiber Bragg Grating (FBG) was proposed. This topology removes the need for a reflector, variable attenuator, or fiber optic beam splitter. The FBG was designed using the OptiGrating software. It was then implemented such that the desired back-reflection level is induced when the FBG is attached to the unisolated DFB laser.

The performance of the DFB laser diode attached to the FBG was analyzed using an Agilent 71400 lightwave signal analyzer. Results were compared to that of the isolated DFB laser diode. Two telecommunications wavelengths, $1310 \mathrm{~nm}$ and $1550 \mathrm{~nm}$, were examined. DFB lasers showed stable spectra, low RIN, and narrow linewidth output when attached to the appropriate FBG. For $1550 \mathrm{~nm}$, the unisolated DFB laser coupled to the FBG achieved a RIN level of $-117.31 \mathrm{~dB} / \mathrm{Hz}$. On the contrary, the isolated DFB laser achieved only $-114.51 \mathrm{~dB} / \mathrm{Hz}$ at a $1 \mathrm{GHz}$ frequency offset. For $1310 \mathrm{~nm}$, the unisolated DFB coupled to the FBG achieved a RIN level of $-158.5 \mathrm{~dB} / \mathrm{Hz}$. This was comparable to $-157.9 \mathrm{~dB} / \mathrm{Hz}$ of the isolated DFB laser operating at a $1 \mathrm{GHz}$ frequency offset for the same optical average power $(5.1 \mathrm{dBm})[54]$. Attaching a FBG to a DFB laser increased the second and third order harmonic distortions by an average of $3 \mathrm{~dB}$ compared to the isolated DFB laser, for both wavelengths.

A pre-distorter model that includes optical back-reflection effects was derived from the laser inverse model using the Voltera series technique. The extracted pre-distorter coefficients were implemented in ADS and cascaded to the laser model to compensate for the laser distortions. Various distortion components were simulated for laser model both with and without the pre-distorter. Simulation results showed that at a 0.10 modulation index, average improvements of $45 \mathrm{~dB}$ in second order harmonic distortion, $55 \mathrm{~dB}$ in third order harmonic distortion, and $40 \mathrm{~dB}$ in various intermodulation distortions can be achieved using the proposed pre-distortion technique. Also at modulation index of 0.04 , the pre-distorter has reduced the second order harmonic distortion and two-tone third order intermodulation distortion levels to values that are lower than $-75 \mathrm{~dB}$ and $-100 \mathrm{~dB}$ respectively, this makes the laser suitable for CATV applications. Although the optical 
back-reflected power is considered one of the major sources for laser noise and nonlinearity, state-of-the-art laser pre-distorters do not account for this effect. Our proposed pre-distorter considerably advances the state-of-the-art by providing a model that is a few steps closer to a comprehensive compensation for the overall laser distortion sources.

\subsection{Future Work}

Future improvements of this work include the following.

- Improved laser model. Incorporating other noise and distortion sources such as laser packaging effect will improve the laser model. The laser package is the interface between the laser module and the rest of the optical system components. It provides mechanical protection and an electrical interface for the laser chip. The laser package also provides the means of removing heat and controlling the laser's temperature. The package parasitics increases distortion and limits the laser bandwidth. Inclusion of packaging parasitic effect in the laser model is highly recommended in order to simulate even more realistic laser performance.

- Better pre-distorter model. The laser pre-distorter model is extracted from the laser model. Hence, including more distortion sources in the laser model will lead to a better distortion compensation capability for the pre-distorter. In addition, increasing the order of the extracted Voltera transfer functions of the pre-distorter will improve the linearity performance.

- Development of an adaptive configuration for the pre-distorter. Adaptive pre-distorters frequently monitor the lasers output in order to update the predistorter coefficients and compensate for any of the lasers' time-varying nonlinearities. As an extension for the pre-distorter model; adaptive algorithms can be used in the pre-distorter model to enable the prediction of the output power variations and hence allows adjusting the pre-distorter coefficients accordingly. Figure 8.1 on the next page shows a block diagram of the suggested adaptive pre-distorter. In this configuration second and third order harmonics of the input signal are generated by appropriate circuits. The feedback path contains a photodiode to monitor the distortion levels and hence update the pre-distorter coefficients to compensate for thermal effects, 
aging, and any other changes.

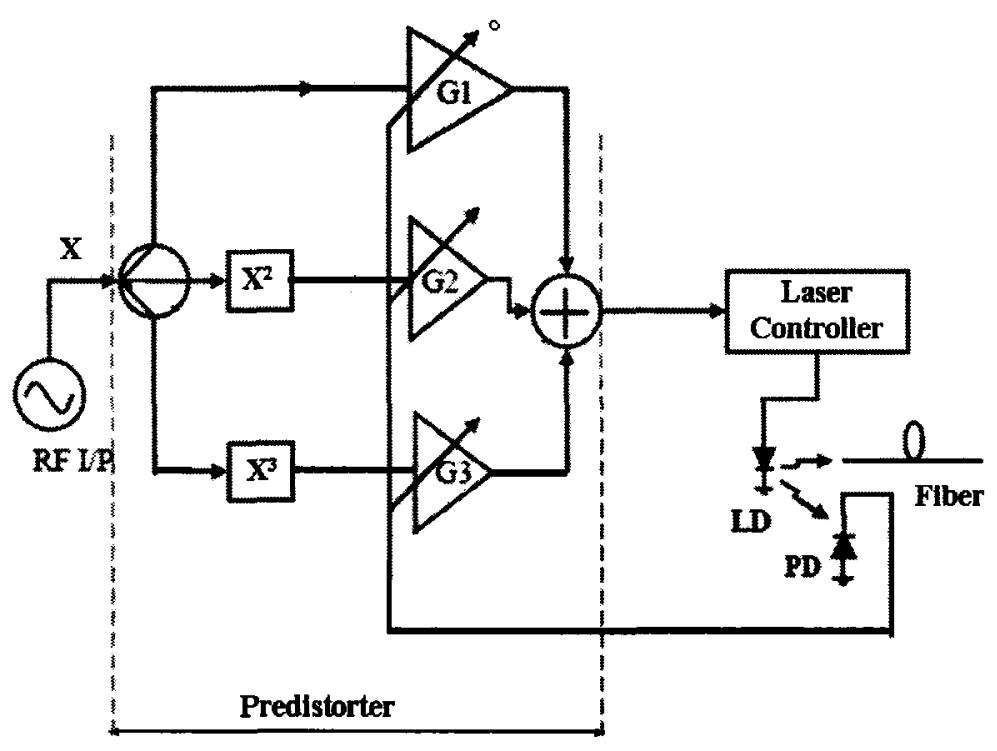

Figure 8.1: Block diagram of the suggested adaptive pre-distorter.

\section{- FPGA (Field Programmable Gate Array) pre-distorter implementation.}

The desired extension of the proposed pre-distorter model involves circuit level implementation. The initial step can be achieved by implementing the pre-distorter using an FPGA board.

The pre-distorter coefficients can be computed, and the model can be described in blocks using suitable software. The laser model and the pre-distorter model have to be implemented using the blocks corresponding to the used FPGA board, the FPGA board can be tested using generated TV signals.

- High frequency and broadband circuit design. As explained in Chapter 7, the pre-distorter contains three branches that need to be designed using three main circuits; the linear circuit to drive the laser, the squaring circuit to provide required second order distortion, and the cubing circuit to provide required third order distortion. The overall circuit of the designed pre-distorter has to be implemented first in software in order to examine the pre-distorter performance. 
- Hardware implementation and fabrication. Pre-distorter hardware implementation is suggested when the designed pre-distorter circuit achieves the desired performance over the required frequency bandwidth, that is when it achieves the desired levels of different harmonic and intermodulation distortion components.

- Pre-distorter testing. Finally, the fabricated pre-distorter circuit can be tested by measuring the laser linearity improvements due to the use of the pre-distorter circuit. This can be achieved by measuring the laser linearity using a lightwave signal analyzer. 


\section{Appendix A}

\section{Measurements Equipments and Results}

\section{A.1 Different Equipments Used in The Measurements}

\section{A.1.1 DFB laser diodes, laser mount and laser controller}

Two DFB laser diodes were used in the measurements: A Laser mate 1550 MQW-DFB pigtailed laser diode and Laser mate $1310 \mathrm{MQW}$-DFB, both had a coaxial structure packaging without an isolator. The ILX lightwave LDC-3724B laser diode controller was used to provide the bias current and the temperature control to the lasers through the THORLABS TCLDM9 5.6mm/9mm Laser diode Mount.

\section{A.1.2 Fiber optic beam splitter}

The OZ FOBS, shown in Figure A.1, was used to divide and couple light in two directions (fibers). It is a two-by-two bi-directional coupler with a 50/50 splitting ratio. It has four ports: $1,2, T$, and $R$. The coupling efficiencies from ports 1 and 2 into port $R$ are similar to those from port $\mathrm{T}$ into ports 1 and 2 . All of its four ports are attached to a single mode fiber pigtail terminated with FC/APC connectors. The FOBS insertion loss is $0.7 \mathrm{~dB}$, the back-reflection level is $-60 \mathrm{~dB}$, and its cross talk level is less than $-40 \mathrm{~dB}$. In the experimental setup, the input from the DFB laser diode goes out through port T. Half of the light is 
coupled into port 1 in order to monitor the source power using OSA, the other half goes through port 2 to the reflector. Port $\mathrm{R}$ couples as much of the back-reflected signal from port 2 , while simultaneously port $\mathrm{T}$ collects the same amount.

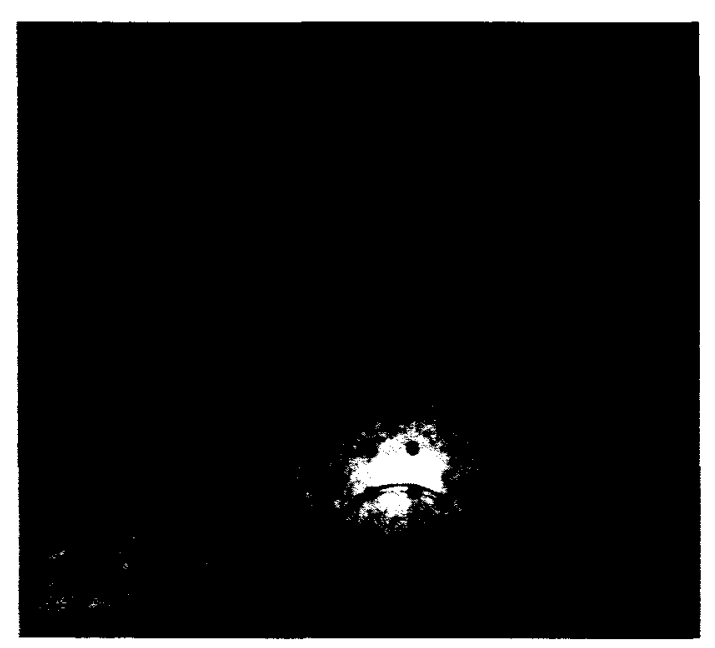

Figure A.1: OZ Optics fiber optic beam splitter with a 50/50 splitting ratio.

\section{A.1.3 Fiber optic reflector}

Figure A.2 shows the OZ Optics Fiber optic reflector. It is a fiber pigtailed reflector terminated with a FC/APC connector that is used to reflect the light. It consists of a fiber optic collimator and a mirror. The output is first collimated, then strikes the mirror and finally reflected back into the collimator. It offers a total reflection for the incident optical power with an insertion loss less than $1.4 \mathrm{~dB}$.

\section{A.1.4 Variable attenuator}

The OZ Optics variable attenuator shown in Figure A.3 consists of two baseplates. Each baseplate contains a fiber followed by a collimating lens. In order to control the level of attenuation, a screw is used to block the collimated beam between the two lenses. to control 


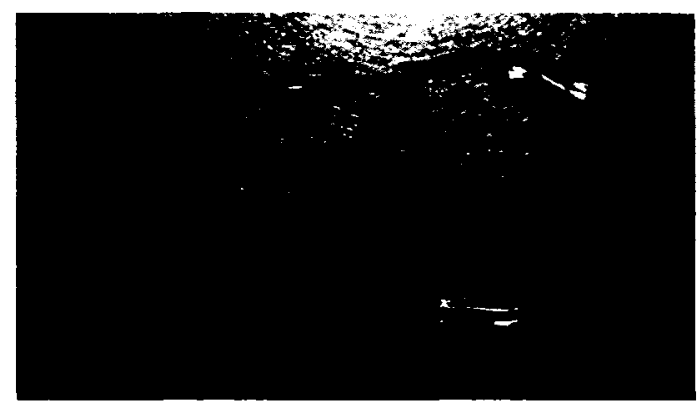

Figure A.2: OZ Optics fiber optic reflector with total reflection.

the attenuation level. The attenuator insertion loss is $-0.75 \mathrm{~dB}$, it offers an attenuation range from its insertion loss up to $80 \mathrm{~dB}$.

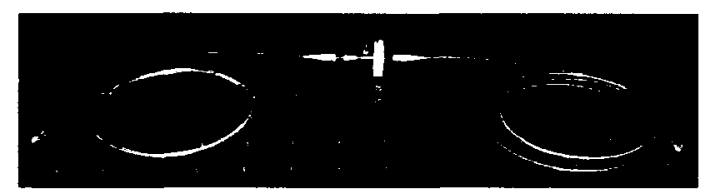

Figure A.3: OZ Optics variable attenuator of attenuation range up to $80 \mathrm{~dB}$.

\section{A.1.5 Optical multimeter}

The ILX Lightwave OMM-6810B Optical Power and Wavelength Meter together with the ILX Lightwave OMH-6745B Fiber Optic power/wavehead shown in Figure A.4 were used in the measurements. These instruments are capable of measuring the optical power and wavelength of semiconductor lasers.

\section{A.1.6 Optical Spectrum Analyzer}

Agilent 86140B Optical Spectrum Analyzer shown in Figure A.5 was used for the required spectral analysis. 


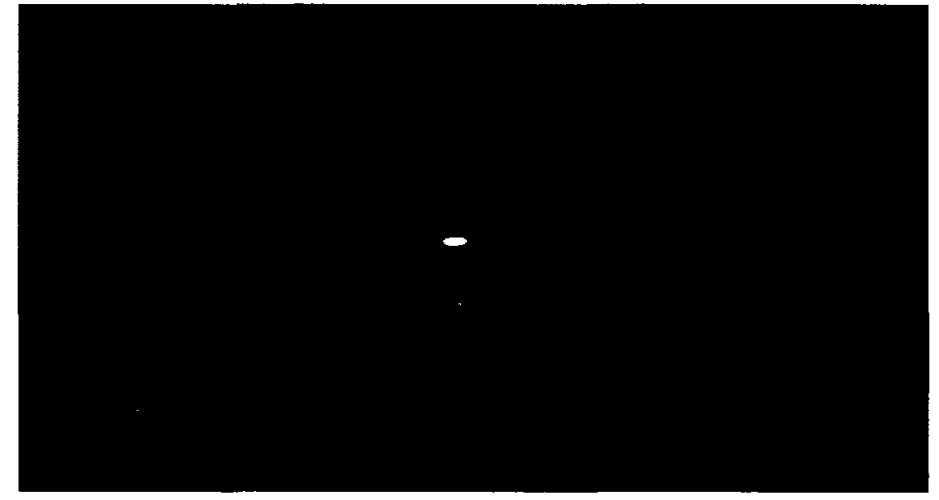

Figure A.4: ILX Lightwave OMM-6810B Optical Power and Wavelength Meter (top) and ILX lightwave LDC-3724B laser diode controller (Bottom).

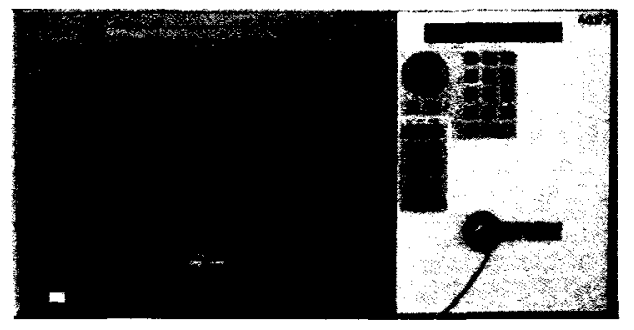

Figure A.5: Agilent 86140B optical spectrum analyzer. 


\section{A.1.7 Polarization controller}

OZ Optics Polarization controller shown in Figure A.6 is used to ensure that the reflected light back into the laser has the same polarization as the emitted light. It has two fiber pigtails at both sides terminated with FC/APC connector. Its insertion loss is less than $0.1 \mathrm{~dB}$.

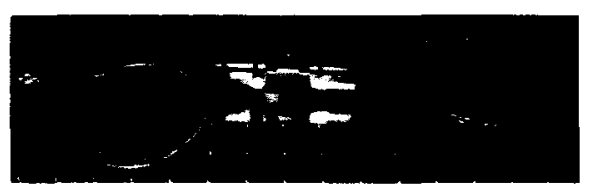

Figure A.6: OZ Optics polarization controller.

\section{A.2 RIN Measurements Results}

Figures A.7 - A.12 on page 150 show the measured average relative intensity noise of a $1310 \mathrm{~nm}$ DFB laser diode for bias current level of $15 \mathrm{~mA}$ and average external reflectivity ranging from $-42 \mathrm{~dB}$ to $-15 \mathrm{~dB}$. 


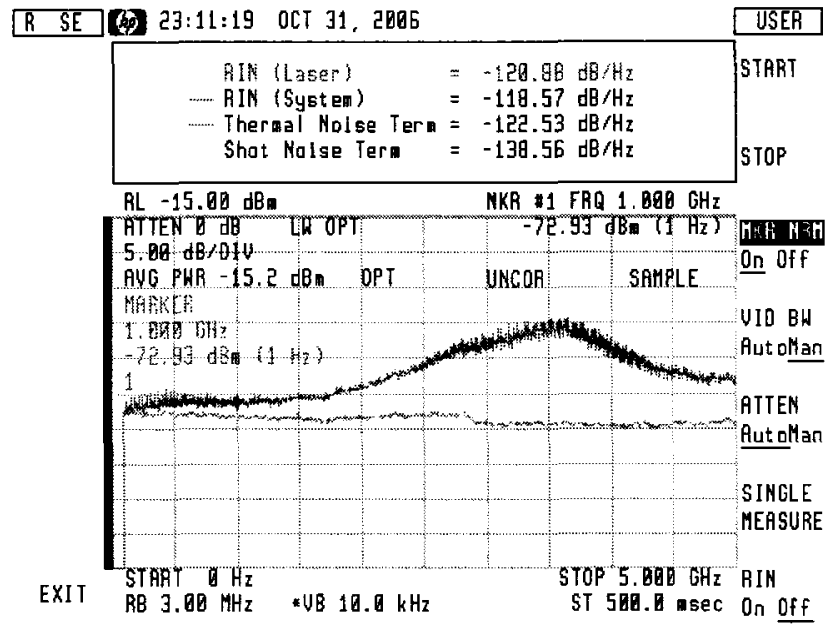

Figure A.7: Measured average relative intensity noise for bias current level of $15 \mathrm{~mA}$ and external reflectivity of $-42 \mathrm{~dB}$.

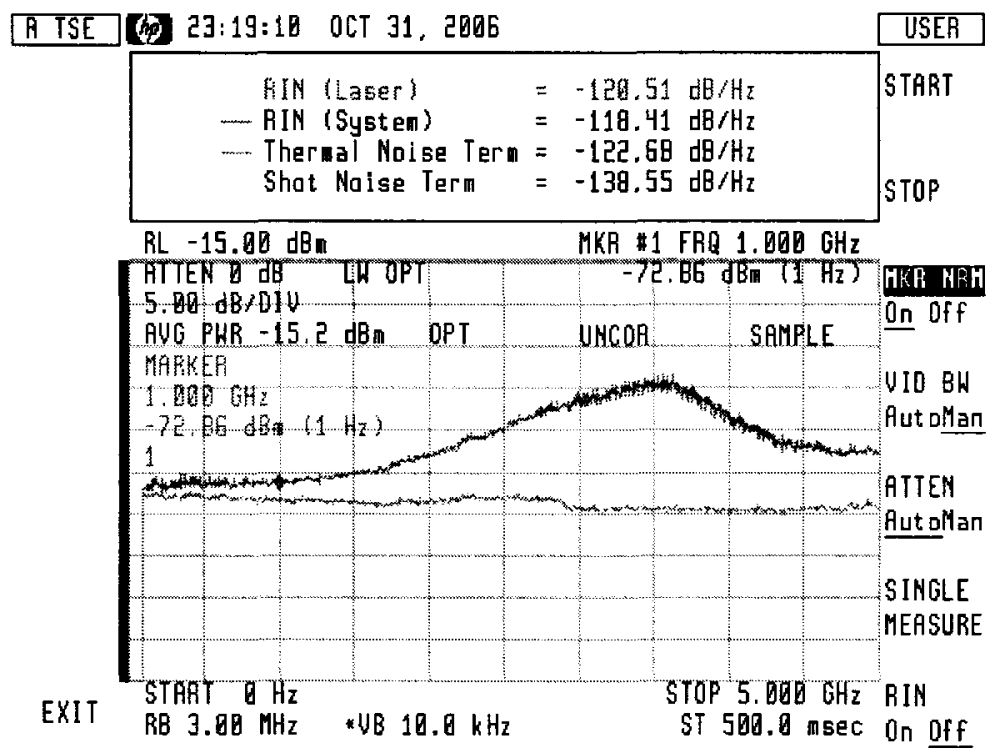

Figure A.8: Measured average relative intensity noise for bias current level of $15 \mathrm{~mA}$ and external reflectivity of $-36 \mathrm{~dB}$. 


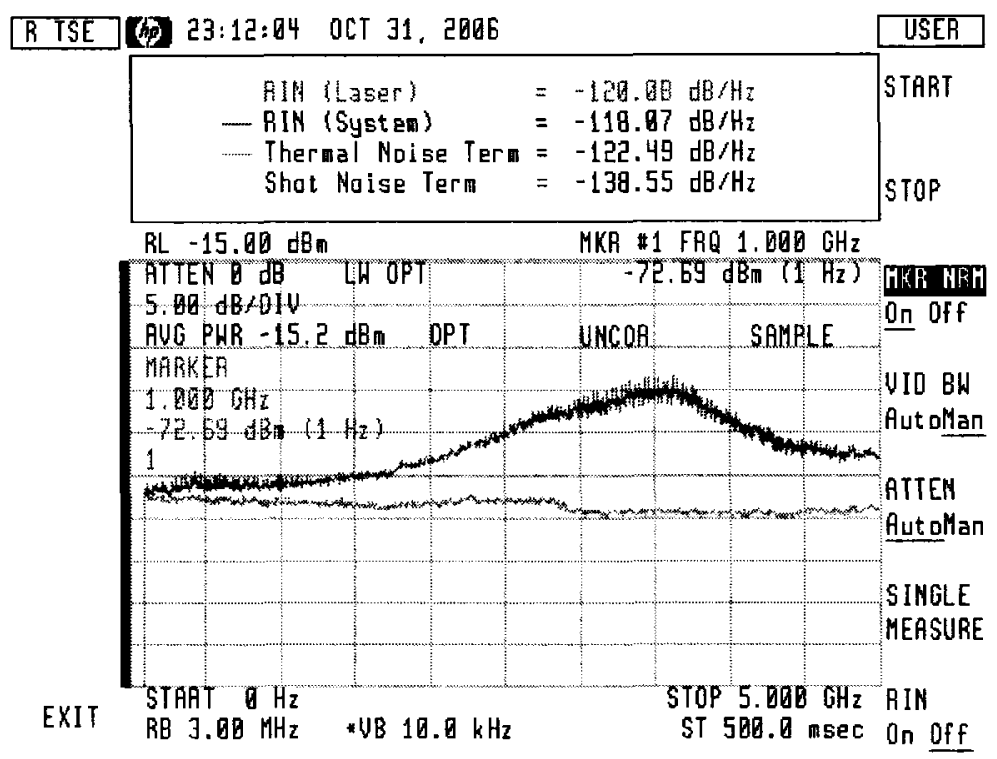

Figure A.9: Measured average relative intensity noise for bias current level of $15 \mathrm{~mA}$ and external reflectivity of $-33 \mathrm{~dB}$.

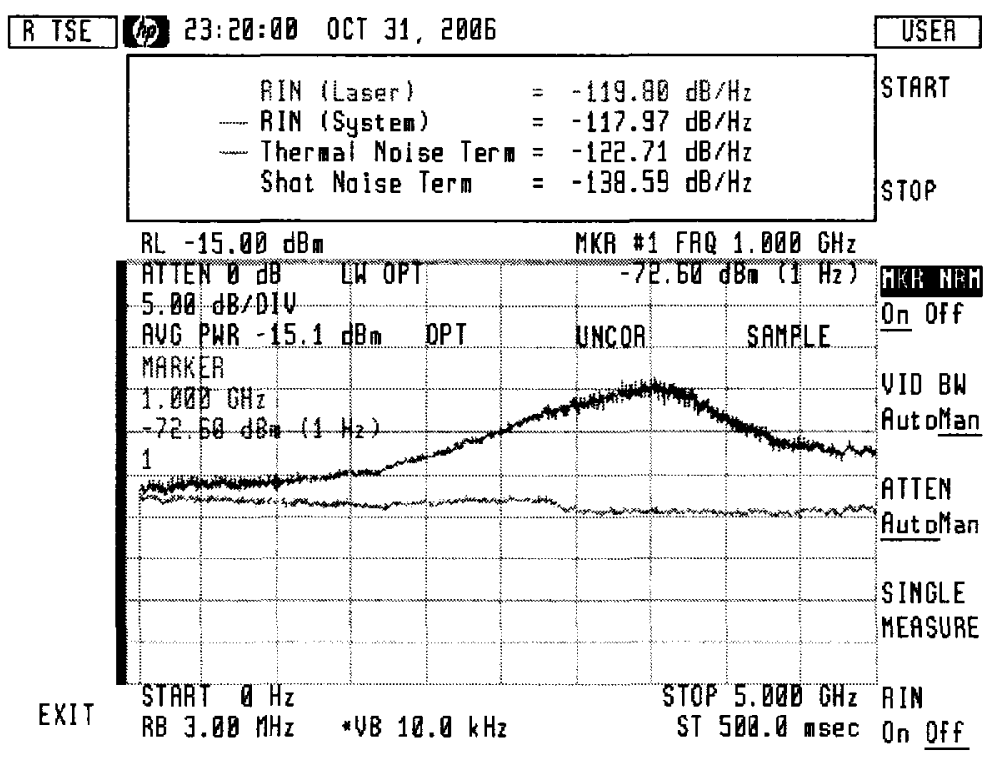

Figure A.10: Measured average relative intensity noise for bias current level of $15 \mathrm{~mA}$ and external reflectivity of $-29 \mathrm{~dB}$. 


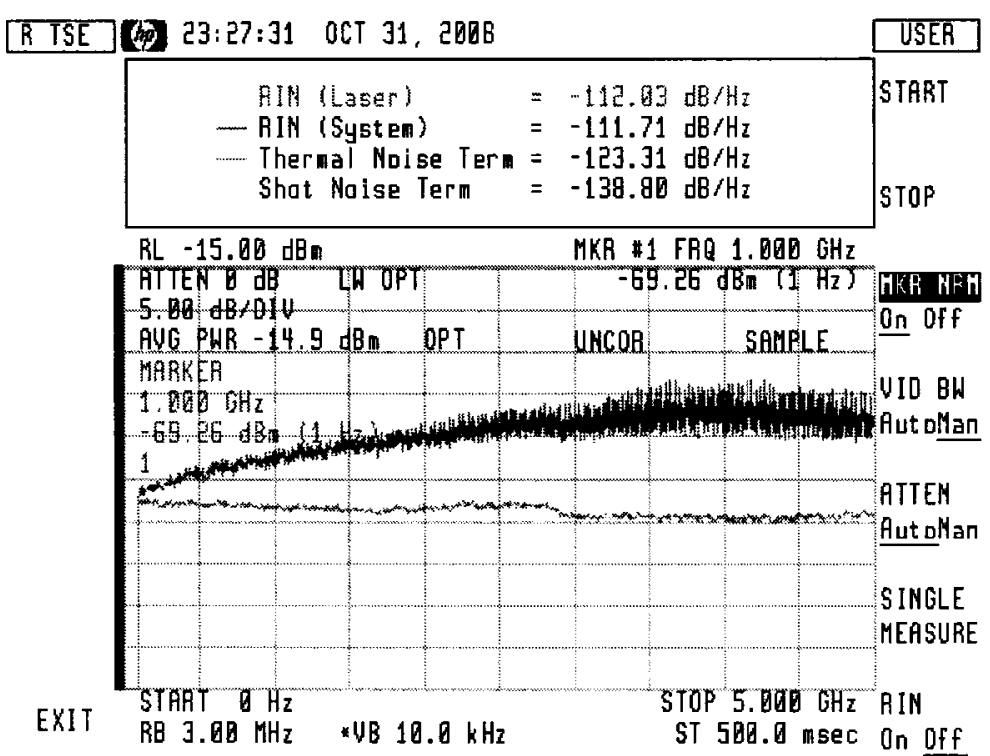

Figure A.11: Measured average relative intensity noise for bias current level of $15 \mathrm{~mA}$ and external reflectivity of $-24 \mathrm{~dB}$.

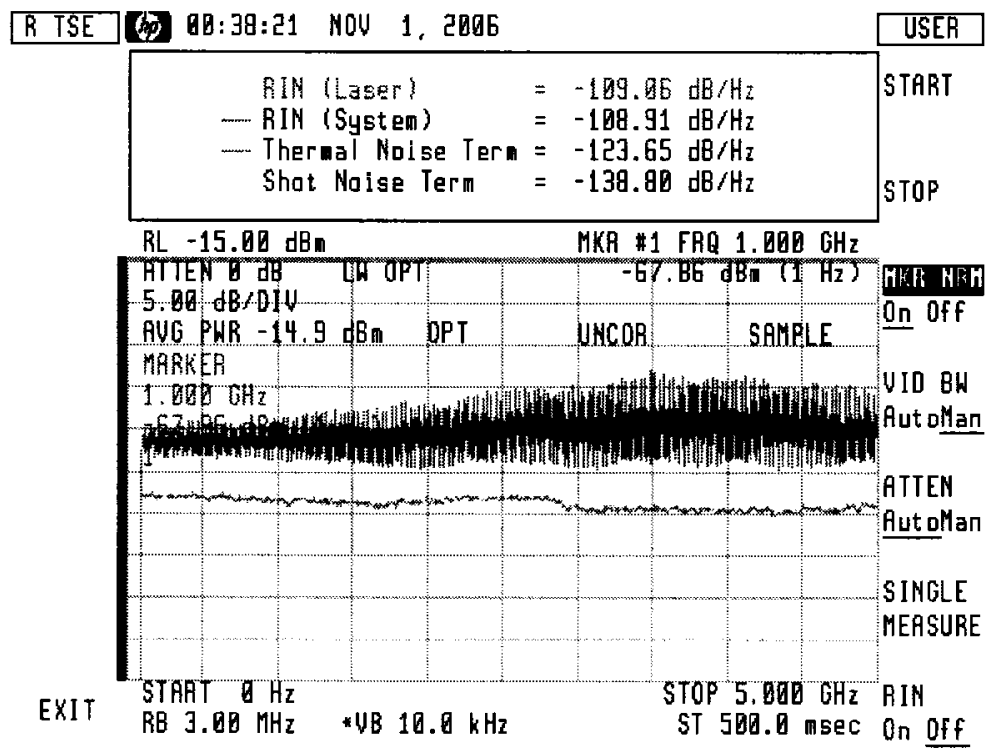

Figure A.12: Measured average relative intensity noise for bias current level of $15 \mathrm{~mA}$ and external reflectivity of $-15 \mathrm{~dB}$. 


\section{Appendix B}

\section{DFB Laser Diode with External FBG}

\section{B.1 FBG Design Steps}

\section{B.1.1 Step 1}

The Optigrating software offers five available modules to work with (Single Fiber, Fiber Coupler, Single Waveguide, Waveguide Coupler, or Other Waveguide), for our application, we will choose Single Fiber.

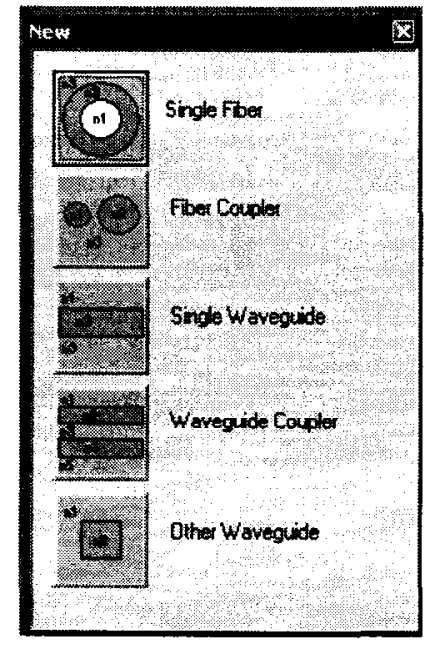

Figure B.1: FBG design step 1 


\section{B.1.2 Step 2}

In this step we will be able to define the following characteristics for the fiber: Index Profile, Photosensitivity Profile, Number Of Points In Mesh, Central Wavelength, etc. We will choose the single fiber core and cladding dimensions to be $4.15 \mu \mathrm{m}$ and $62.5 \mu \mathrm{m}$ respectively and the central wavelength to be $1553 \mathrm{~nm}$.

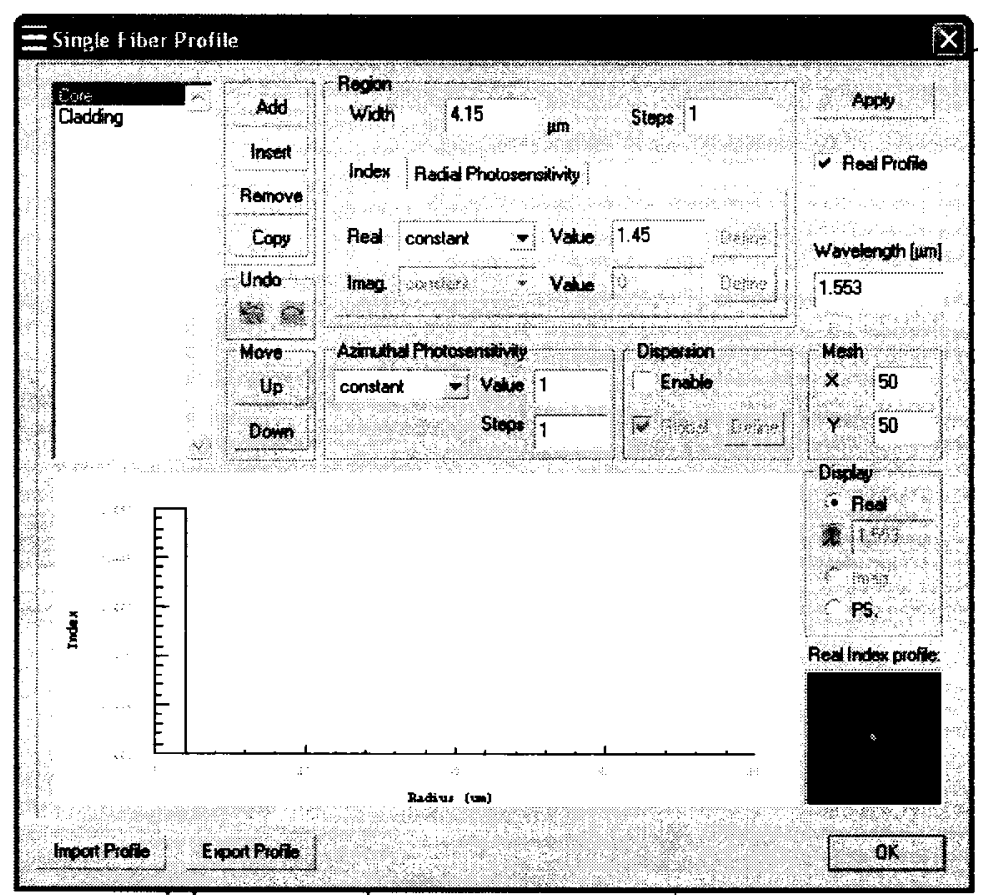

Figure B.2: FBG design step 2

\section{B.1.3 Step 3}

The fiber modes can be set in this step. OptiGrating gives us access to a list of the calculated modes of the fiber structure. 


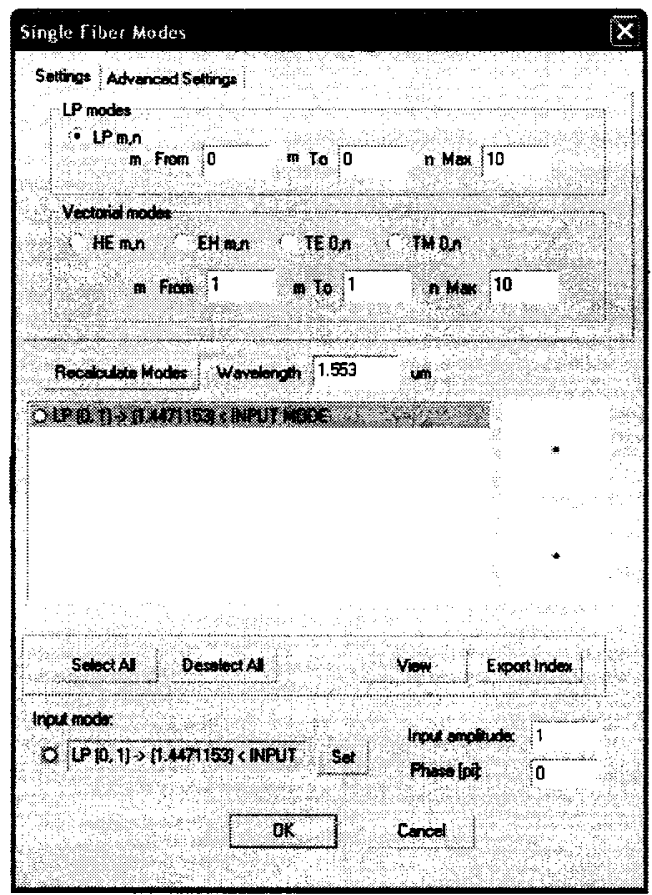

Figure B.3: FBG design step 3 


\section{B.1.4 Step 4}

In this step, the parameters of the grating were defined. Based on the above calculations and the desired characteristics of the grating, the grating parameters are as follows: sine grating shape, uniform average index, no chirp, uniform apodization, $0.5365 \mu \mathrm{m}$ grating period, $10 \mathrm{~cm}$ grating length, $3.075 \times 10^{-5}$ index modulation which is equivalent to $\kappa L=0.4812$.

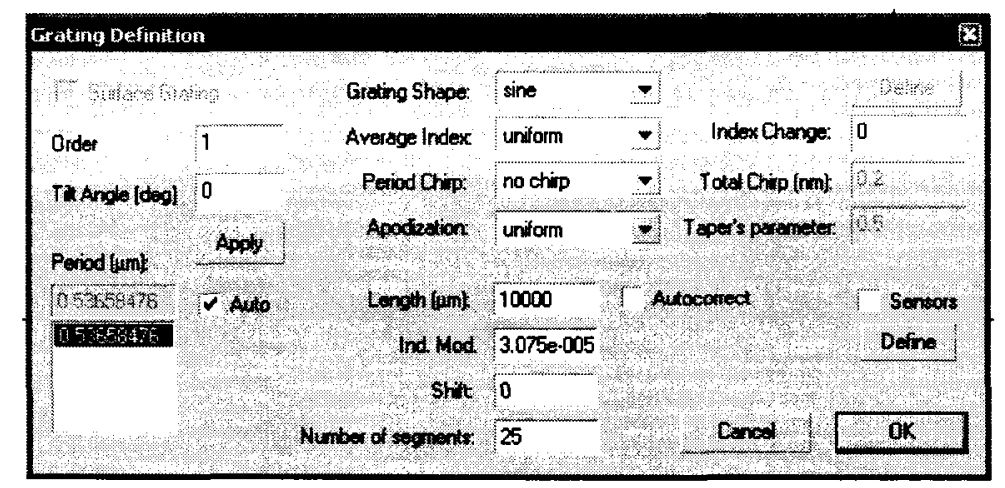

Figure B.4: FBG design step 4

\section{B.1.5 Step 5}

After defining all the necessary parameters for the FBG, we are now ready to work with the multiple view window and access different parameter buttons to examine our designed FBG. Figure B.5 shows the transmission (red curve) and the reflection (blue curve) characteristics of the designed FBG.

\section{B.1.6 Step 6}

The FBG FWHM 'full width at half maximum' bandwidth can be measured automatically using OptiGrating software. Figure B.6 shows the measurements of the designed FBG FWHM bandwidth, which was found to be $0.0792 \mathrm{~nm}$. 


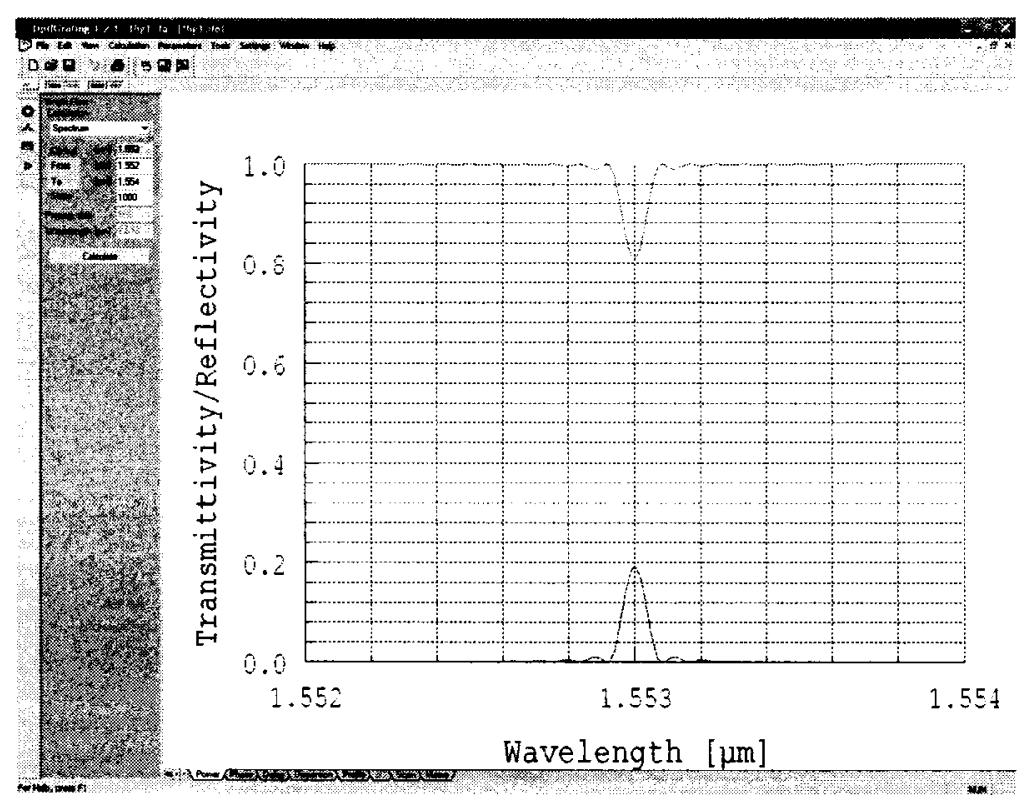

Figure B.5: FBG design step 5

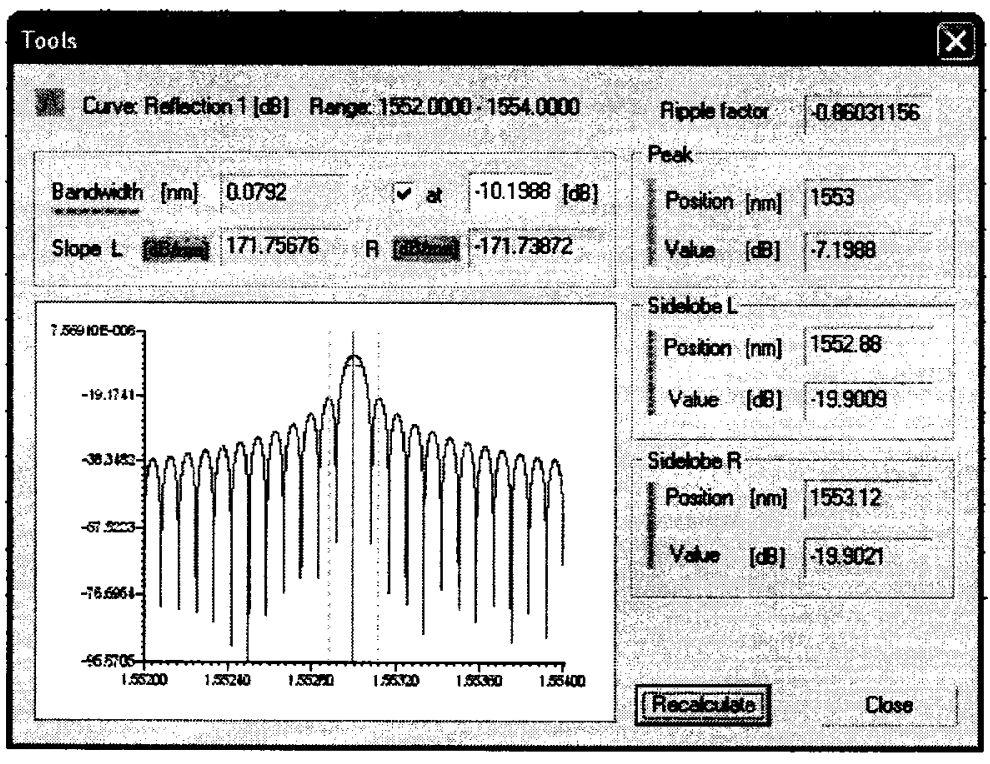

Figure B.6: FBG design step 6 


\section{B.2 FBG Transmission and Reflection Characteris- tics}

Figures B.7 - B.12 on page 162 show different implemented FBGs with the indicated reflection and transmission characteristics. 

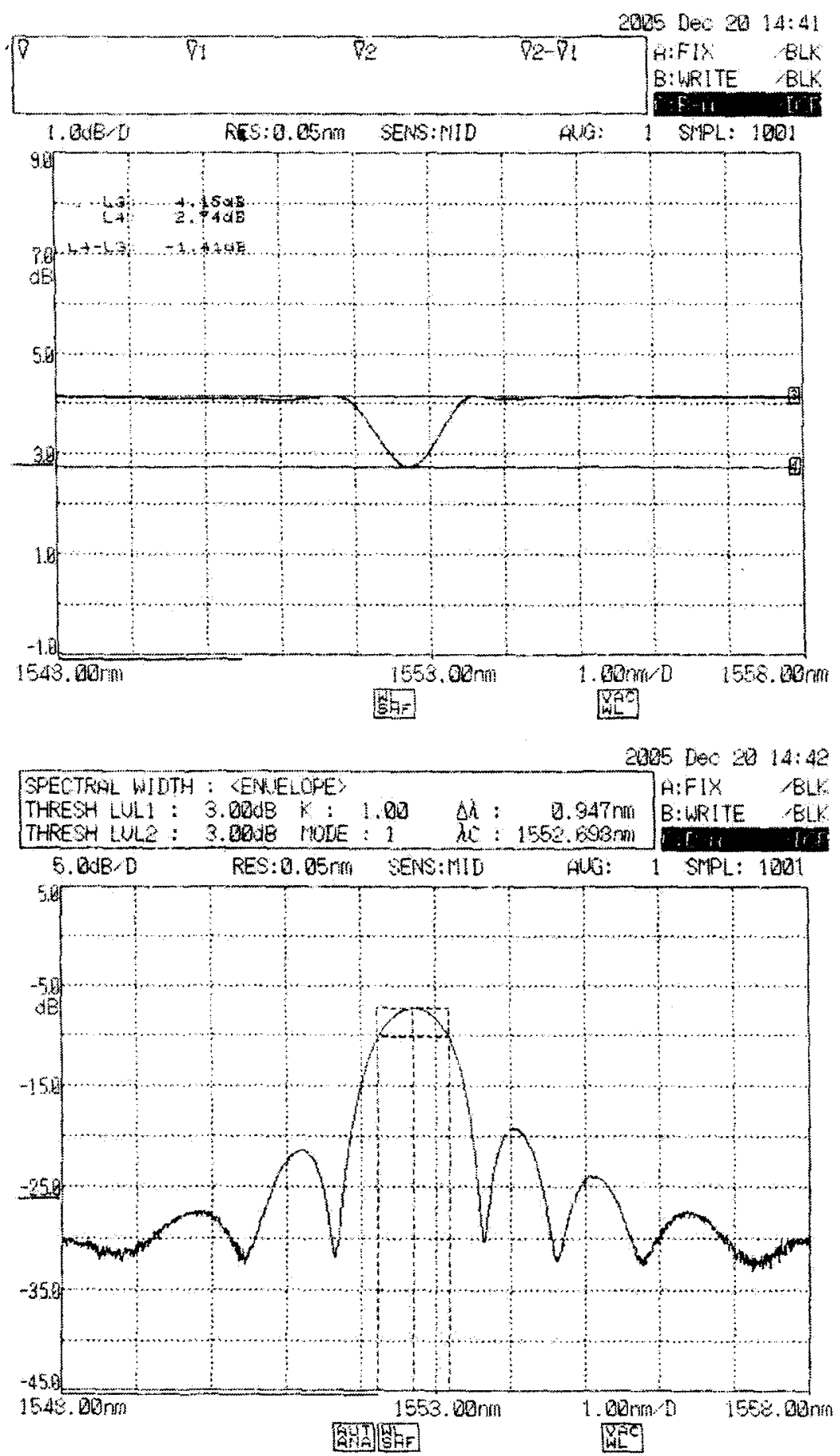

Figure B.7: The implemented FBG2 transmission (top) and reflection (bottom) characteristics. 

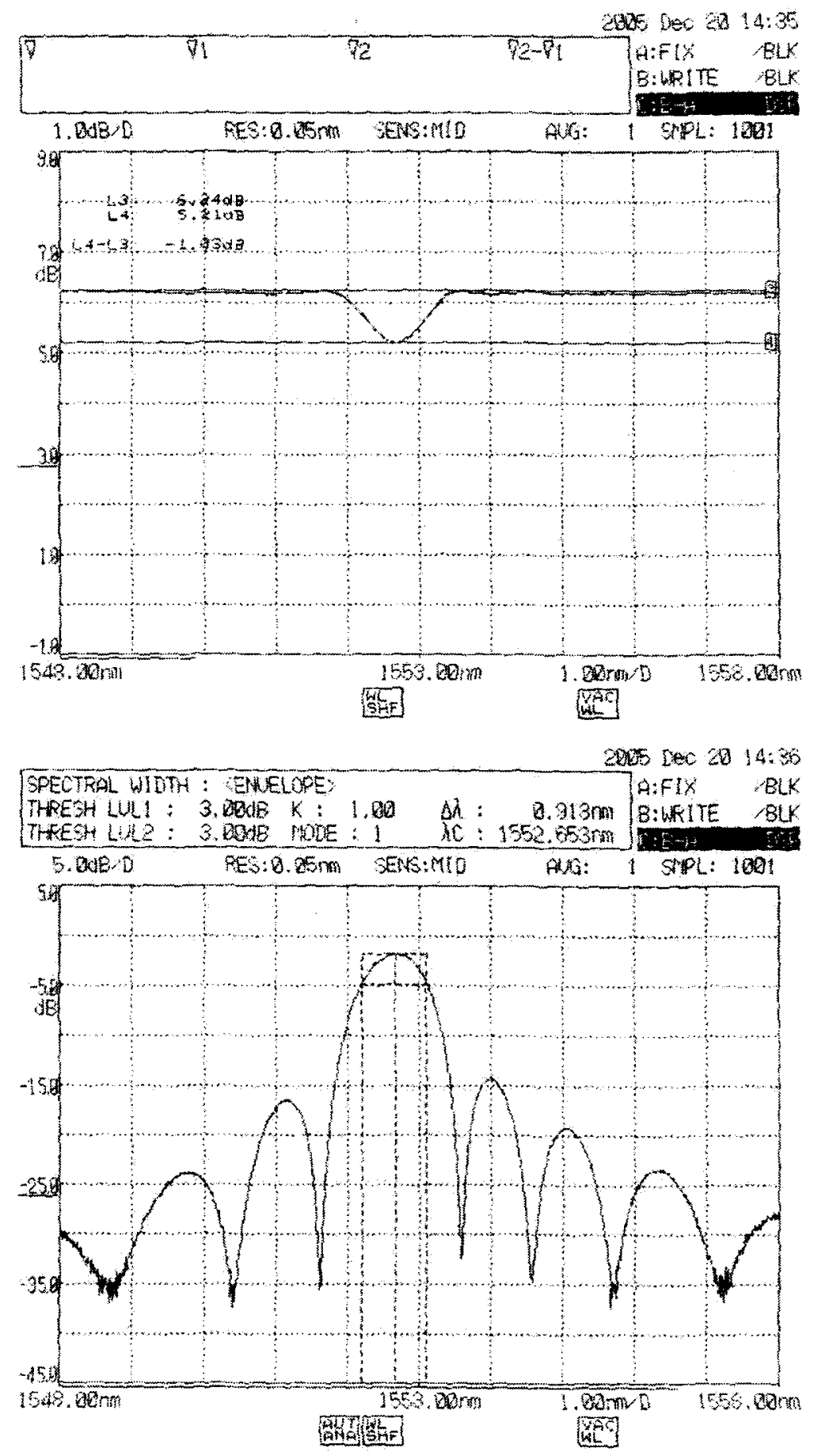

Figure B.8: The implemented FBG4 transmission (top) and reflection (bottom) characteristics. 
2006 feb $2810: 20$

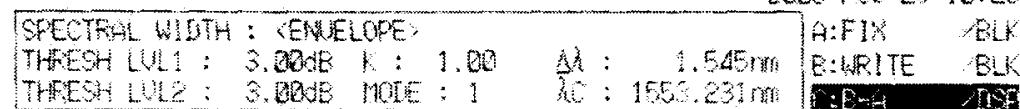

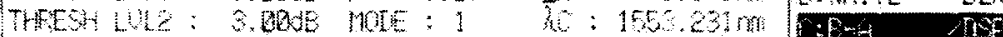

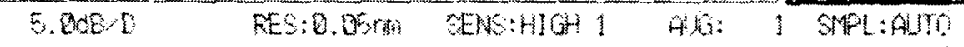

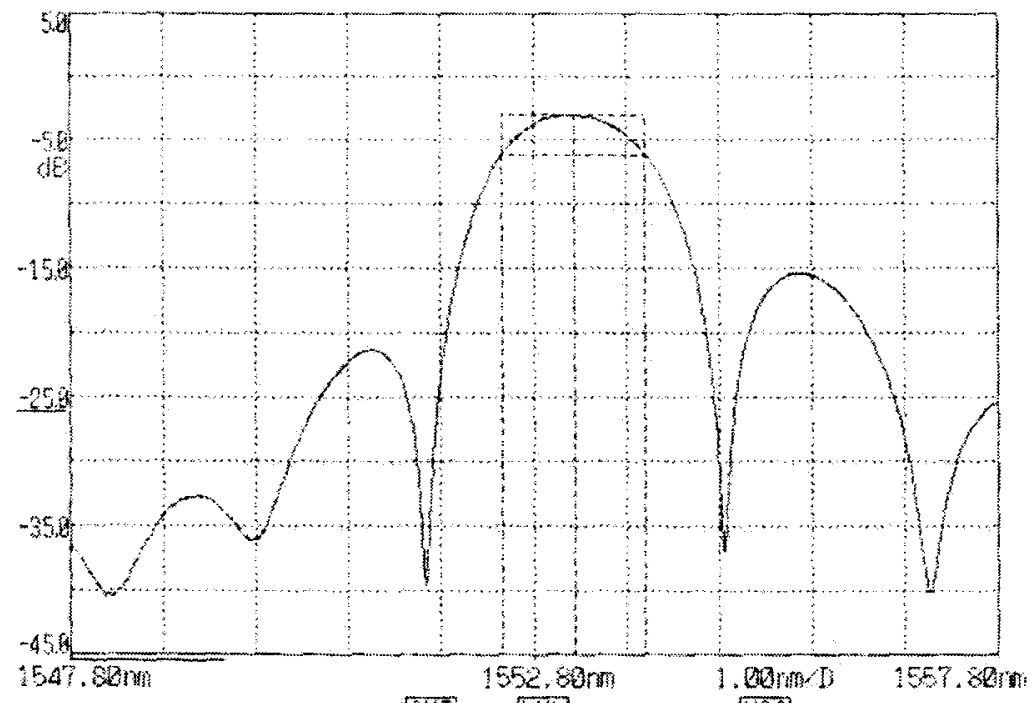

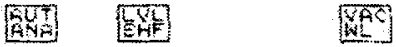
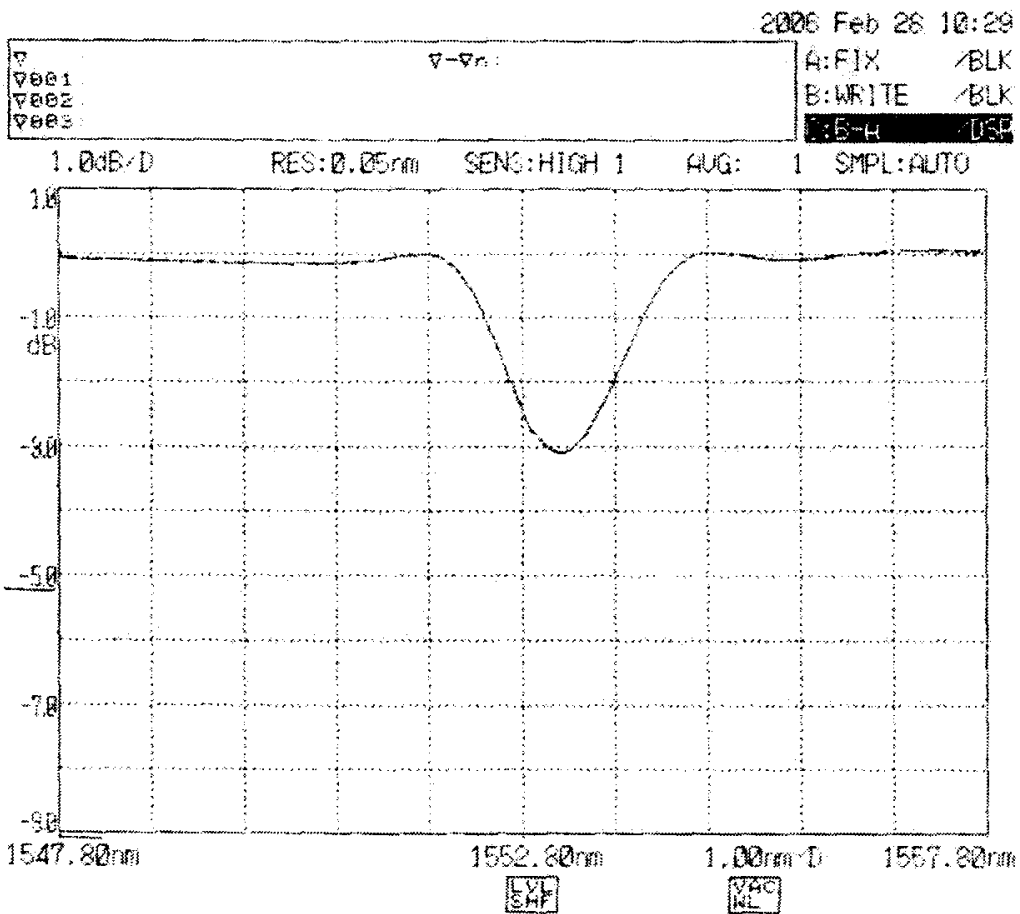

Figure B.9: The implemented FBG7 reflection (top) and transmission (bottom) characteristics. 

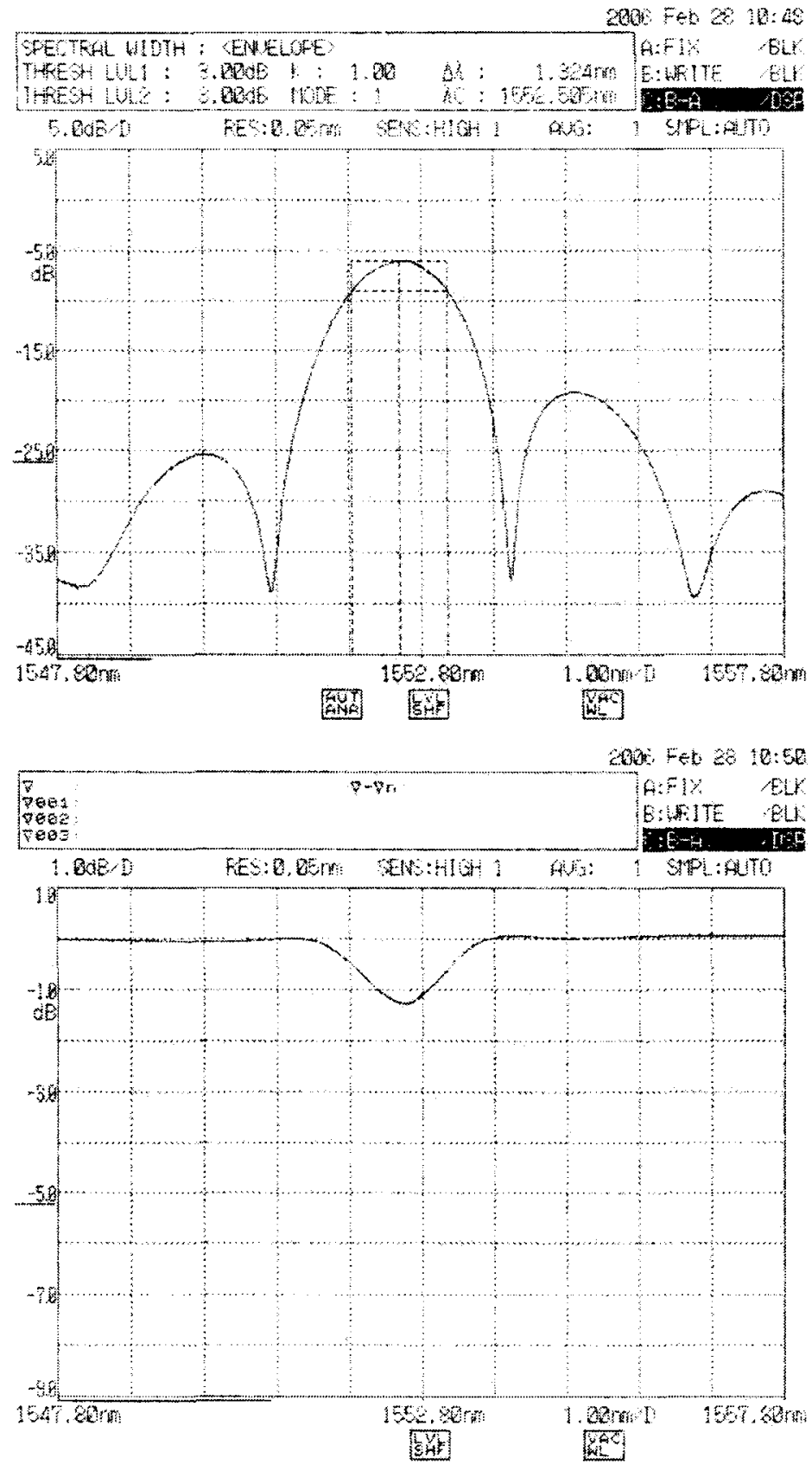

Figure B.10: The implemented FBG10 reflection (top) and transmission (bottom) characteristics. 


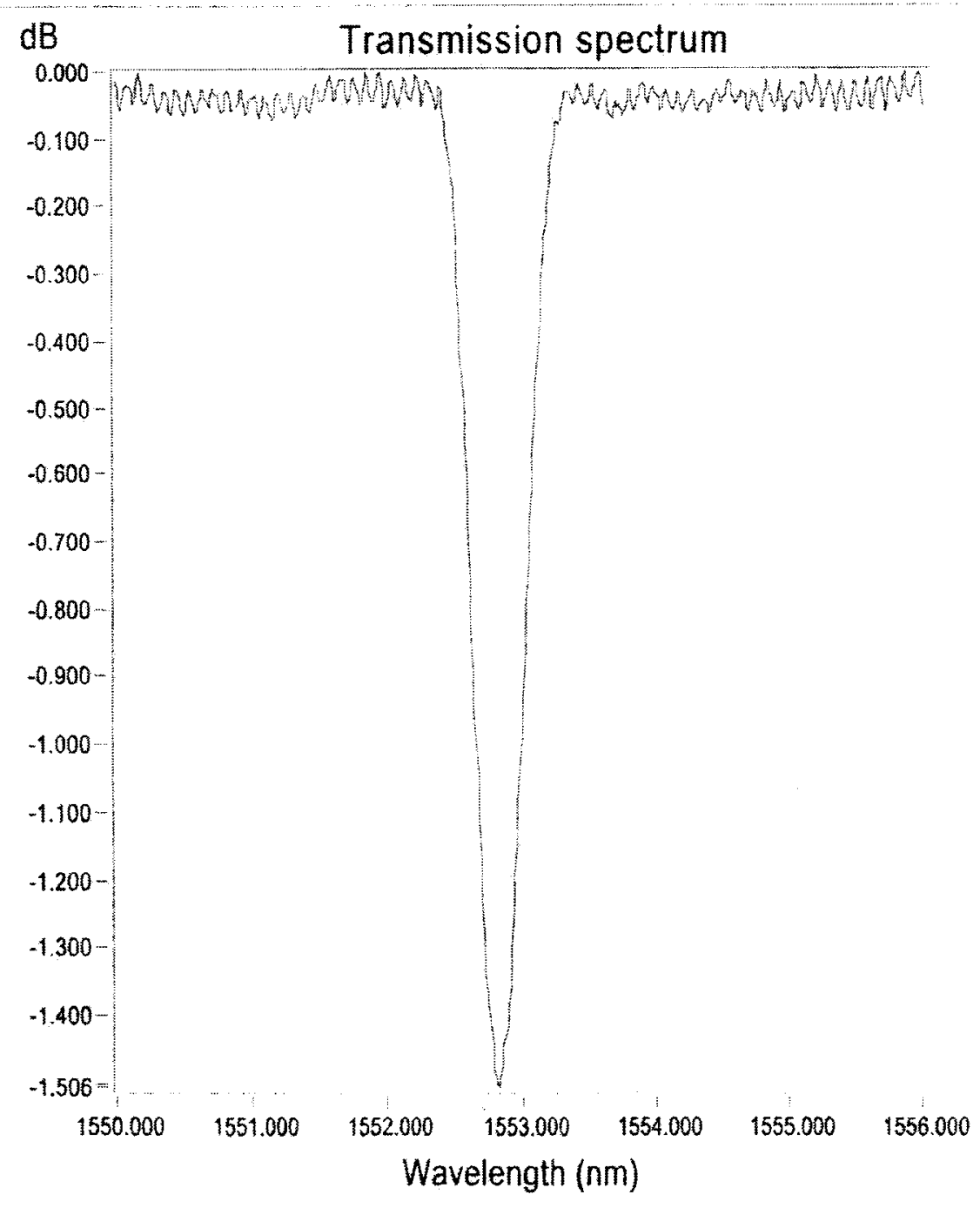

Figure B.11: The FBG11 transmission characteristics. 


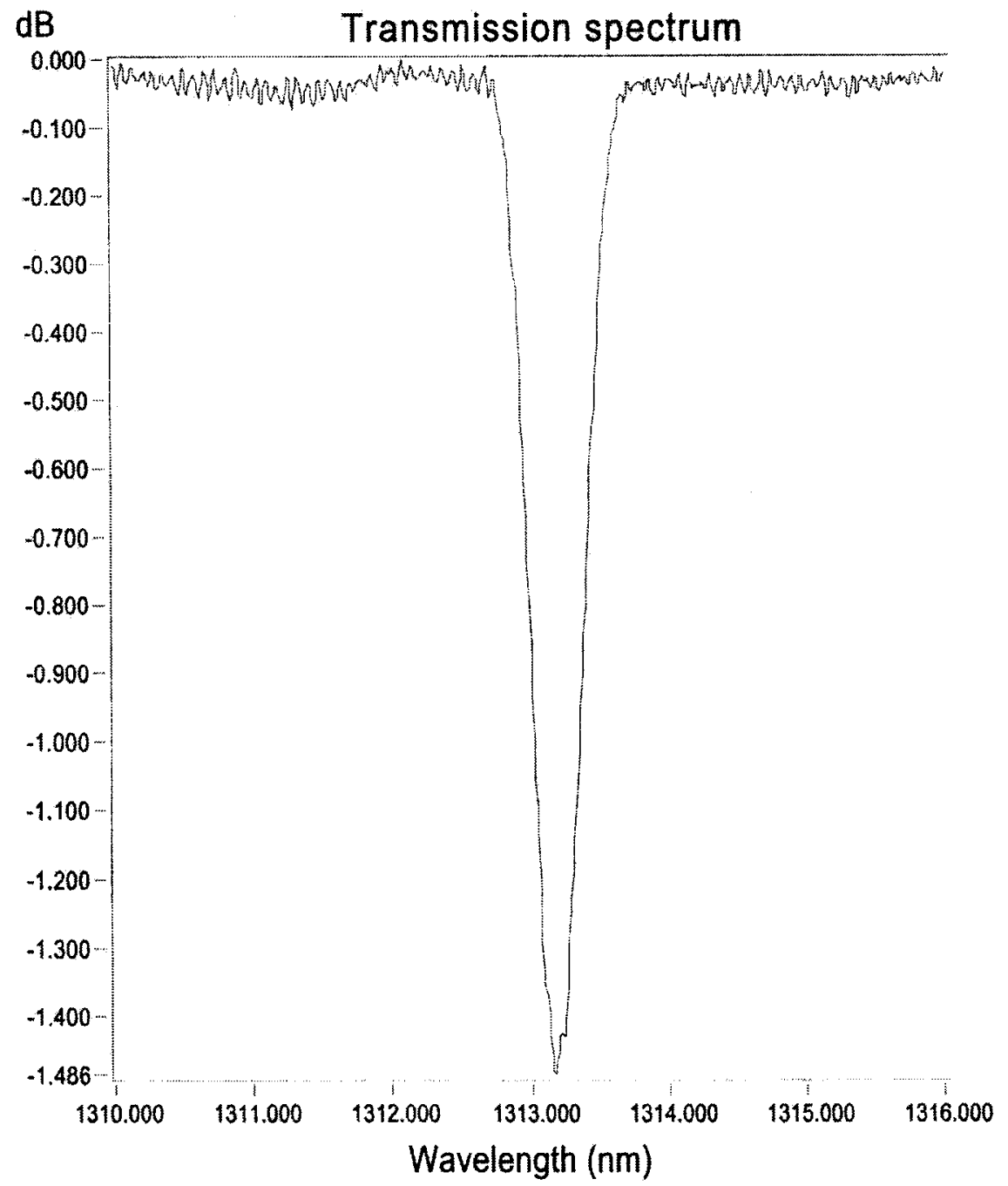

Figure B.12: FBG12 transmission spectrum at central wavelength of $1313.19 \mathrm{~nm}$. 


\section{B.3 Spectrum Stability of DFB Laser with FBG}

Figures B.13 - B.18 on page 166 show different spectra of DFB5 attached to various FBGs and biased at the indicated bias current.

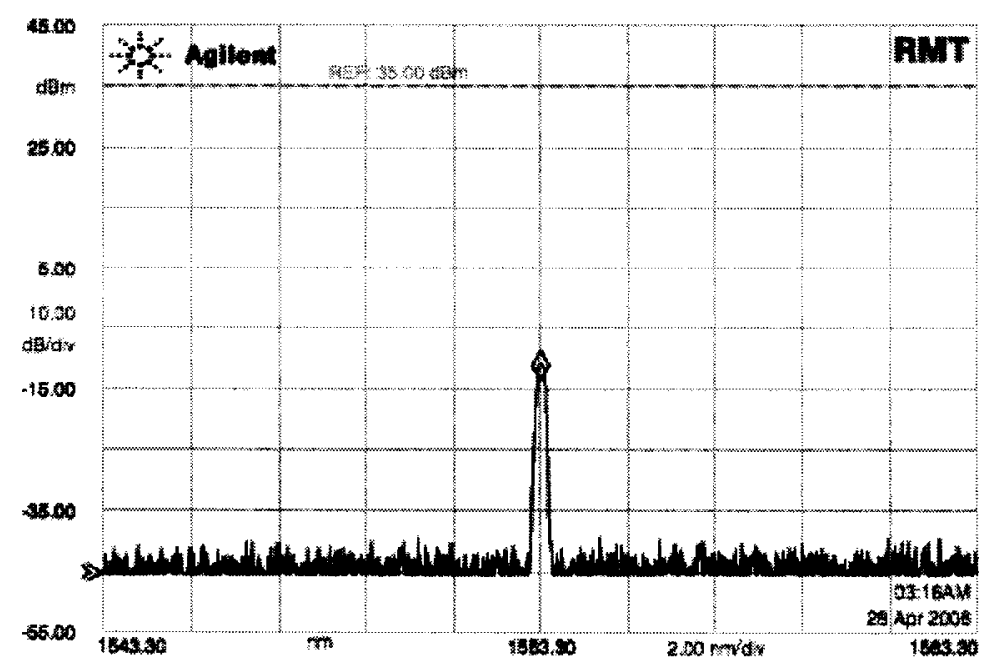

Figure B.13: Spectrum of DFB5 attached to FBG4 operating at bias level of $15 \mathrm{~mA}$. 


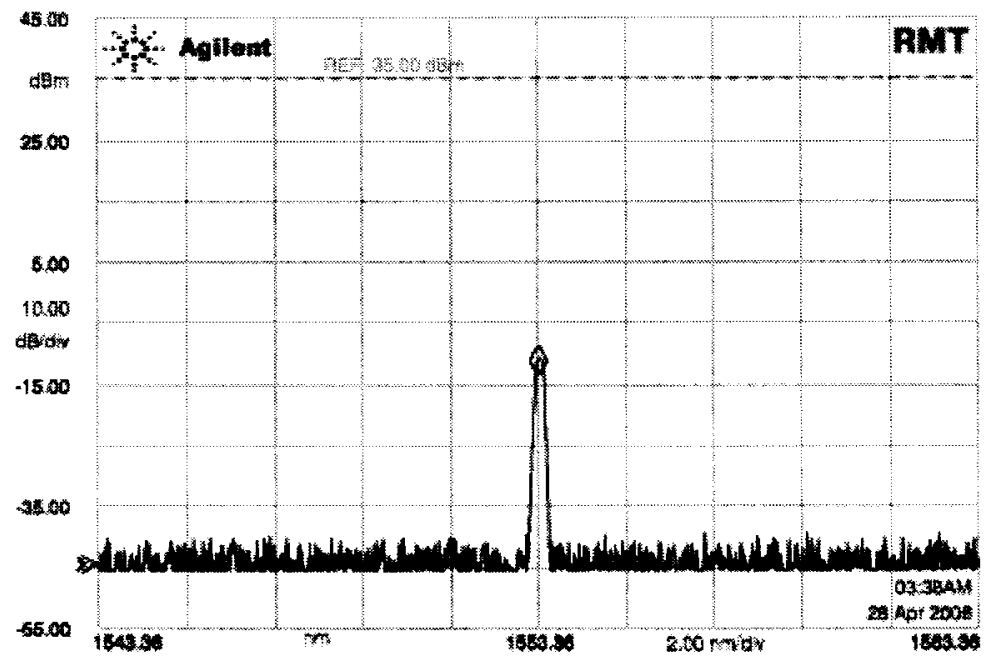

Figure B.14: Spectrum of DFB5 attached to FBG7 operating at bias level of $15 \mathrm{~mA}$.

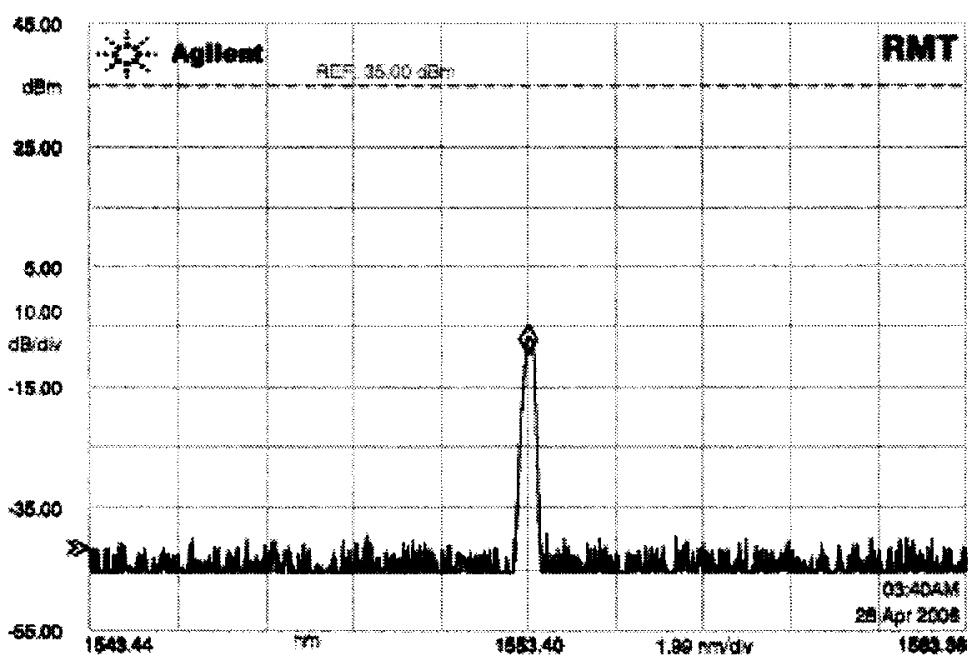

Figure B.15: Spectrum of DFB5 attached to FBG7 operating at bias level of $20 \mathrm{~mA}$. 


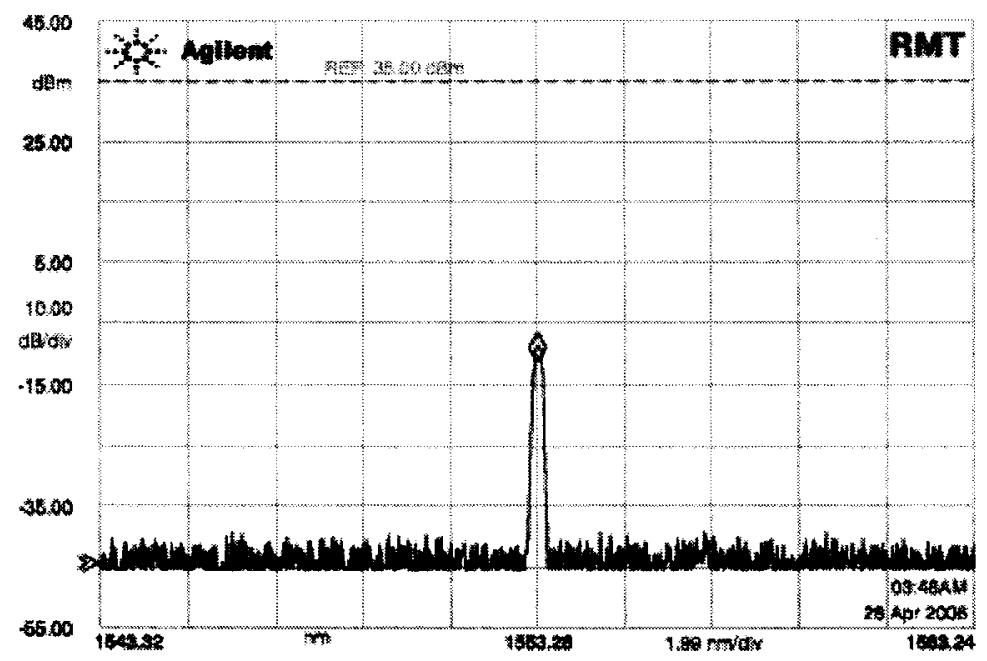

Figure B.16: Spectrum of DFB5 attached to FBG10 operating at bias level of $15 \mathrm{~mA}$.

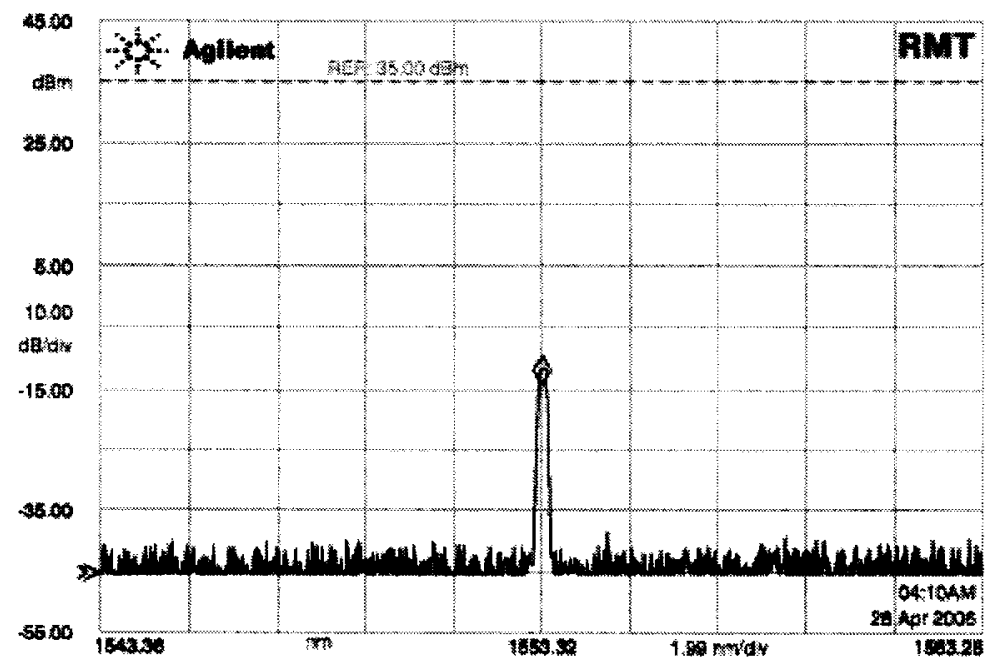

Figure B.17: Spectrum of DFB5 attached to FBG11 operating at bias level of $15 \mathrm{~mA}$. 


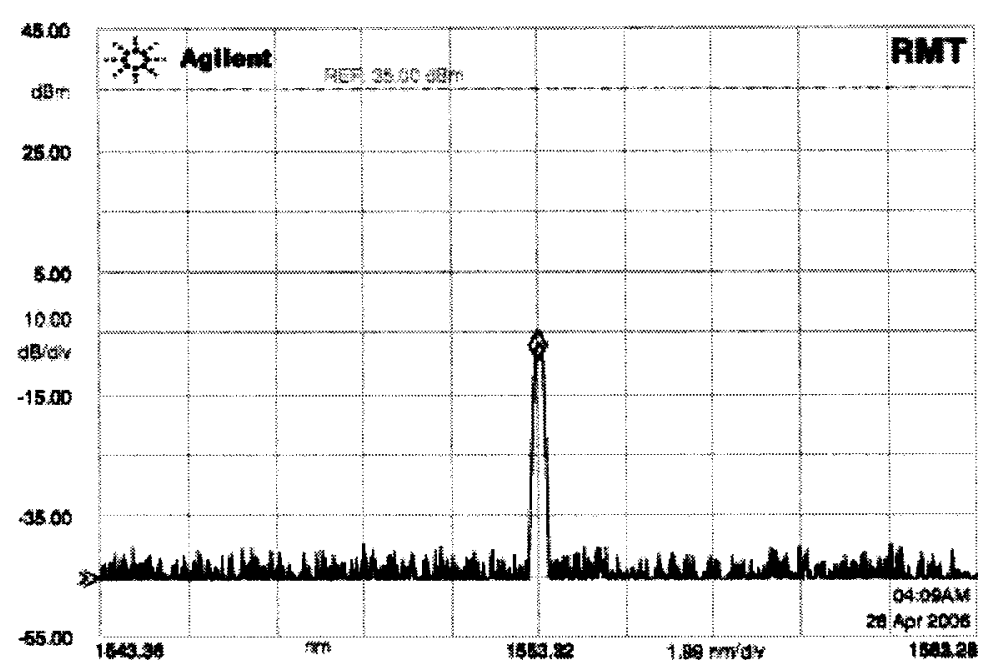

Figure B.18: Spectrum of DFB5 attached to FBG11 operating at bias level of $20 \mathrm{~mA}$.

\section{B.4 RIN Measurements of DFB Laser with FBG}

Figures B.19 and B.20 on the next page show measured average RIN of DFB5 biased at $15 \mathrm{~mA}$ and attached to FBG1 and FBG5 respectively. 


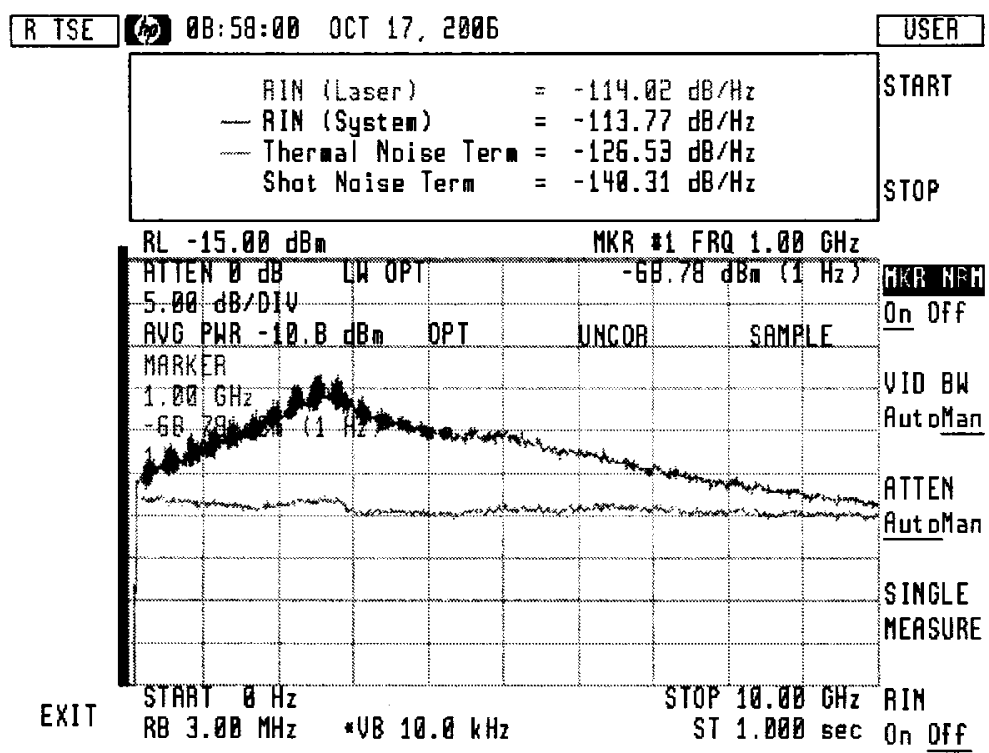

Figure B.19: Measured RIN of DFB5 attached to FBG1 operating at bias level of $15 \mathrm{~mA}$.

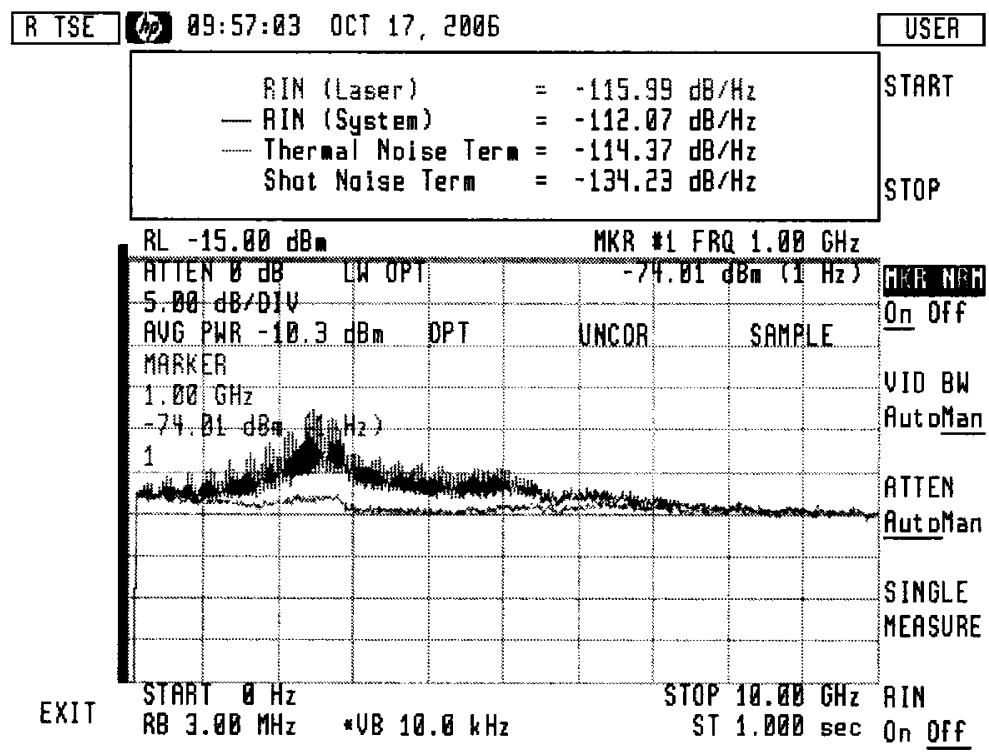

Figure B.20: Measured RIN of DFB5 attached to FBG2 operating at bias level of $15 \mathrm{~mA}$. 


\section{B.5 Distortion Measurements of DFB Laser with FBG}

Figures B.21 - B.28 on page 172 show measured second and third order harmonic distortions of DFB5 attached to various FBGs and operating at the indicated conditions.

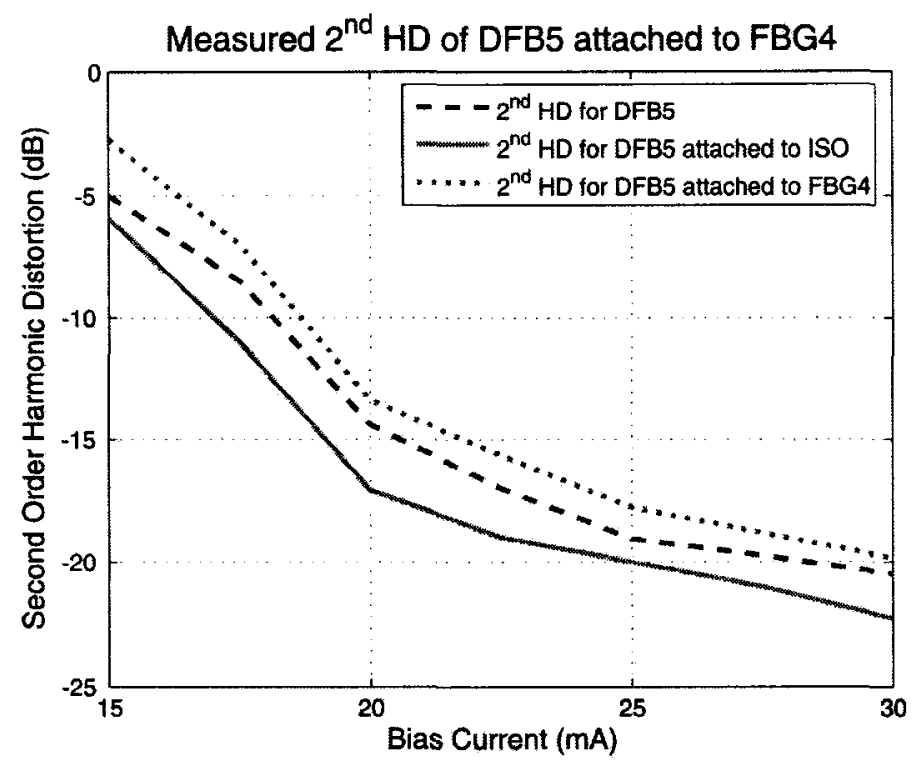

Figure B.21: Measured second order harmonic distortion normalized to the fundamental frequency of DFB5 attached to FBG4. 


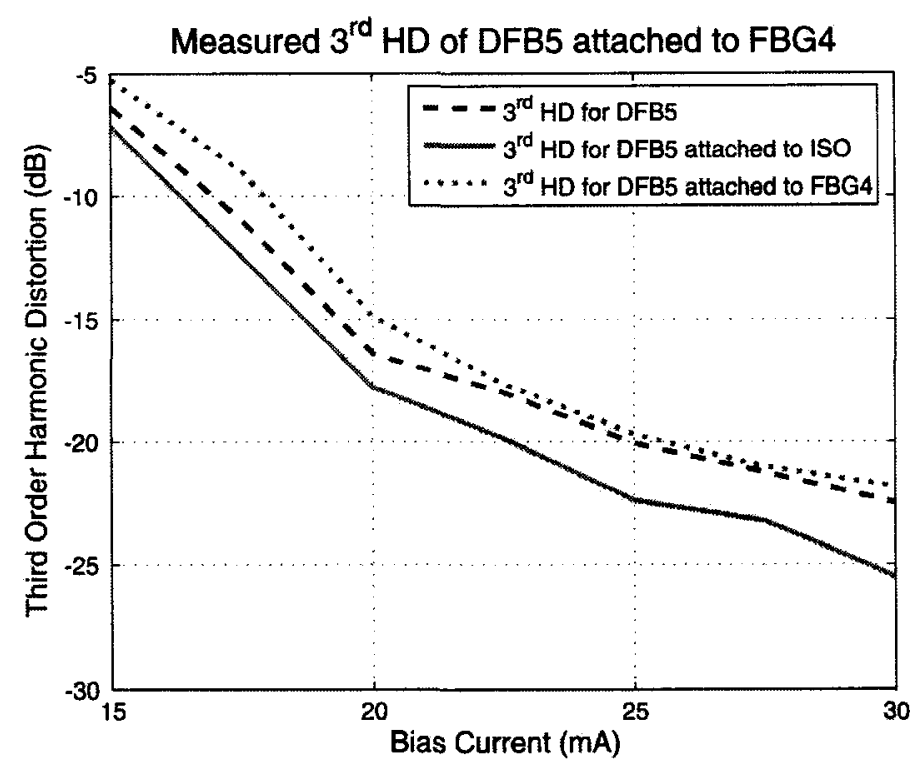

Figure B.22: Measured third order harmonic distortion normalized to the fundamental frequency of DFB5 attached to FBG4.

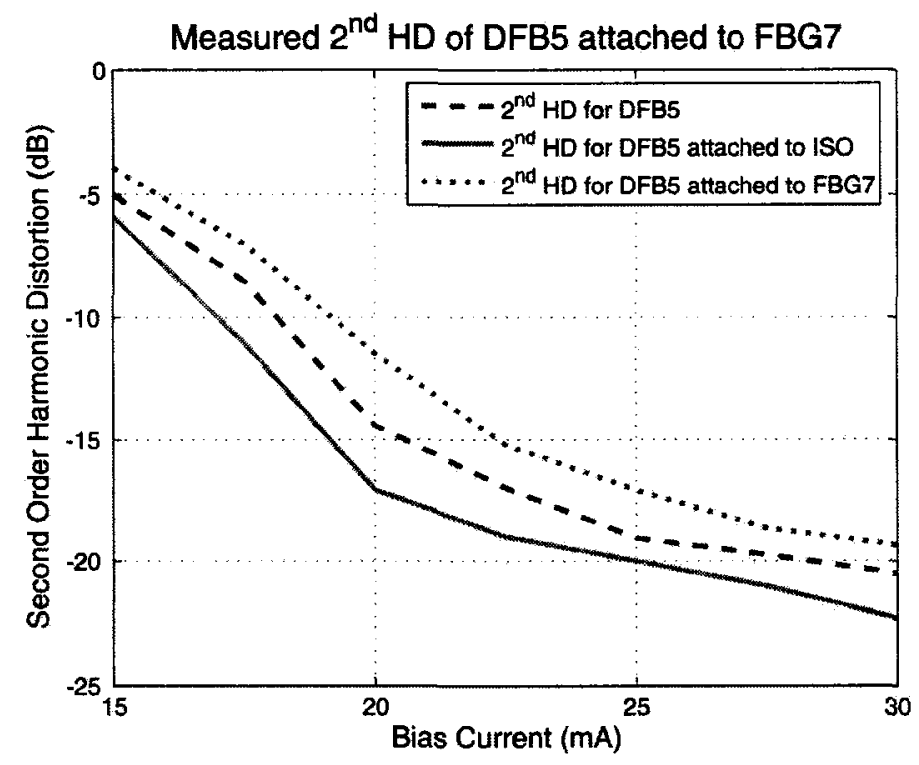

Figure B.23: Measured second order harmonic distortion normalized to the fundamental frequency of DFB5 attached to FBG7. 


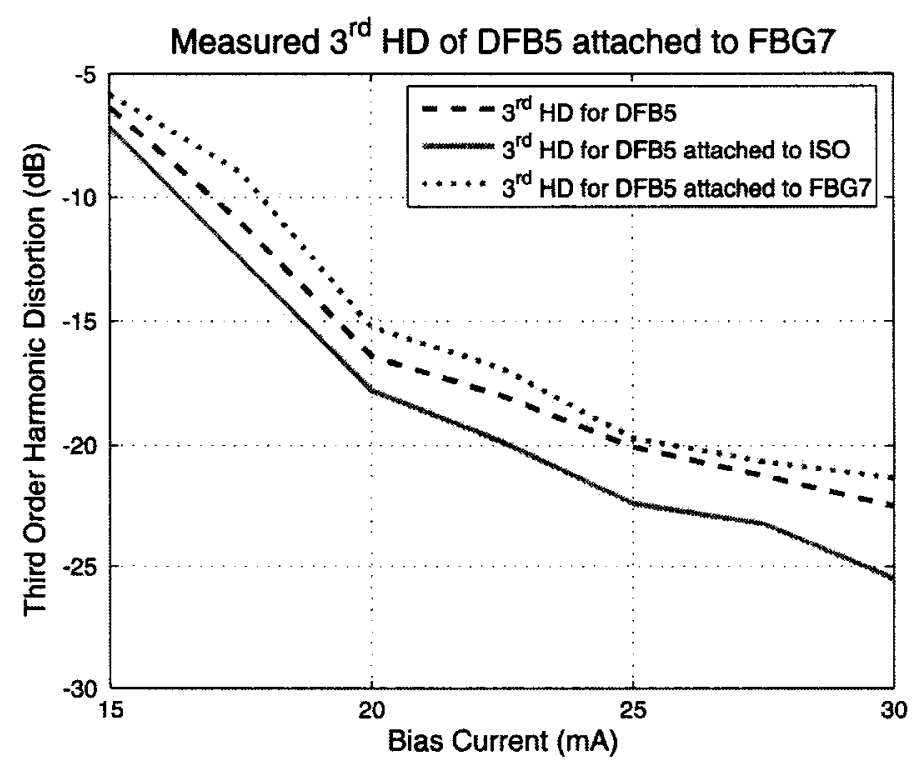

Figure B.24: Measured third order harmonic distortion normalized to the fundamental frequency of DFB5 attached to FBG7.

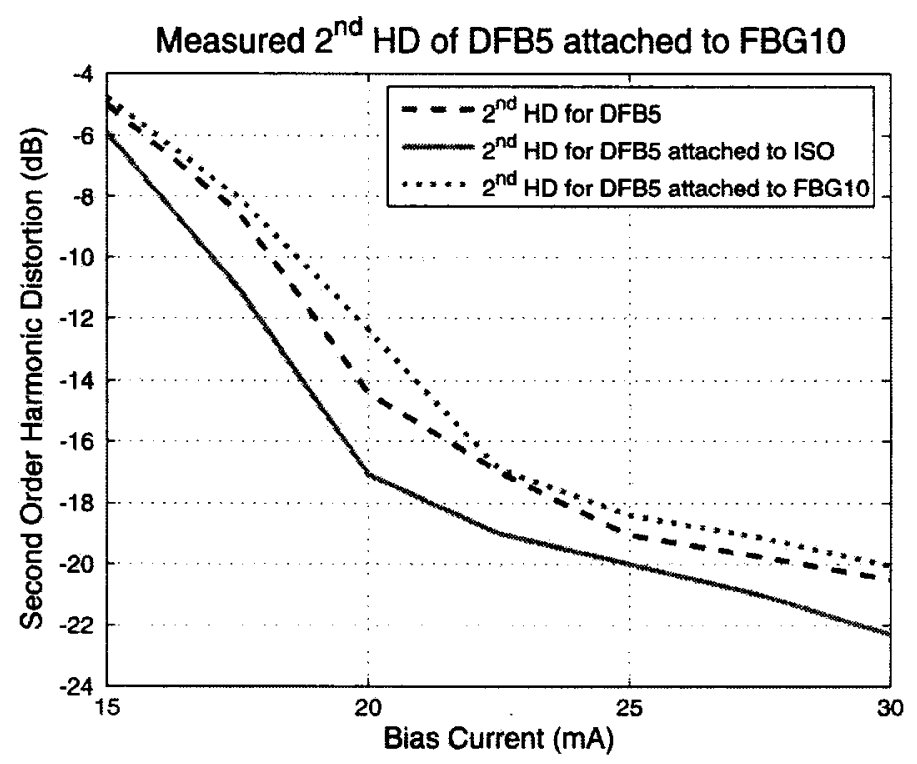

Figure B.25: Measured second order harmonic distortion normalized to the fundamental frequency of DFB5 attached to FBG10. 


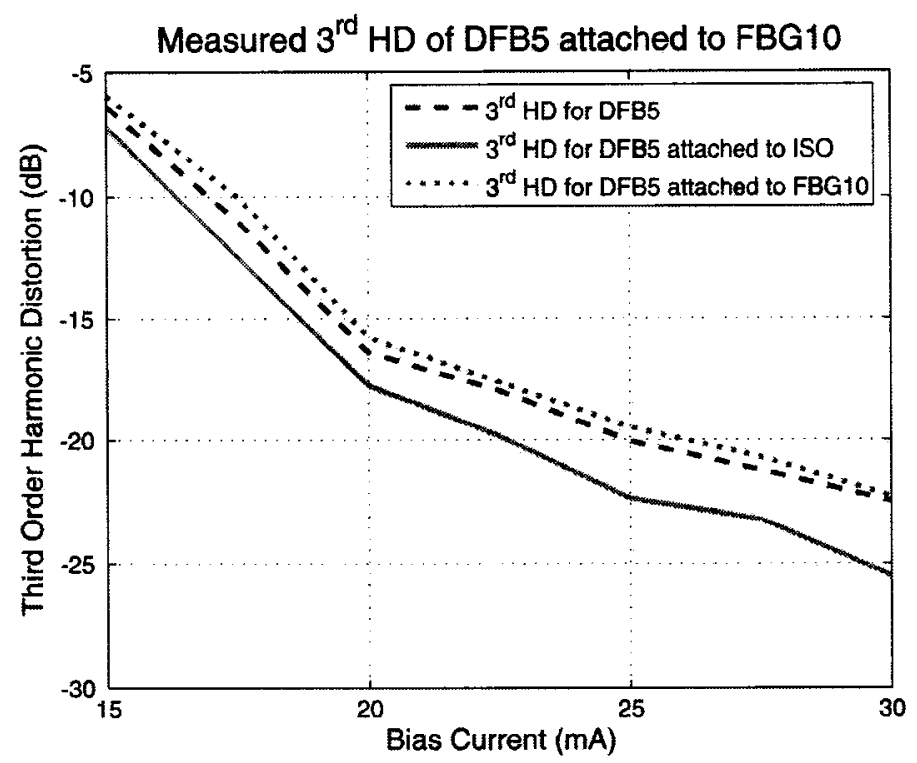

Figure B.26: Measured third order harmonic distortion normalized to the fundamental frequency of DFB5 attached to FBG10.

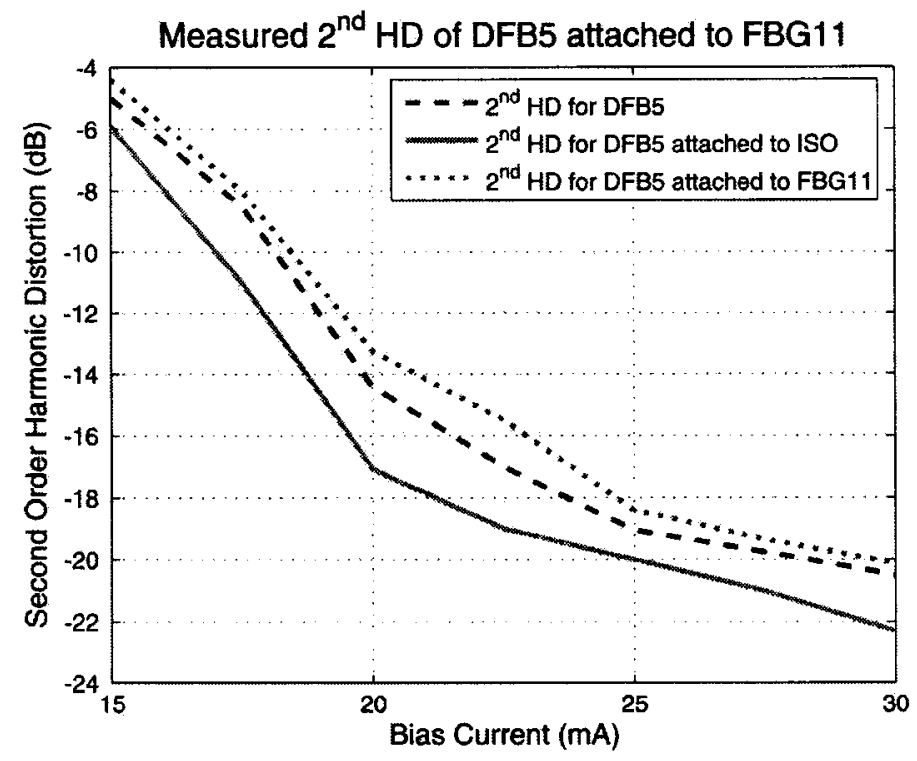

Figure B.27: Measured second order harmonic distortion normalized to the fundamental frequency of DFB5 attached to FBG11. 


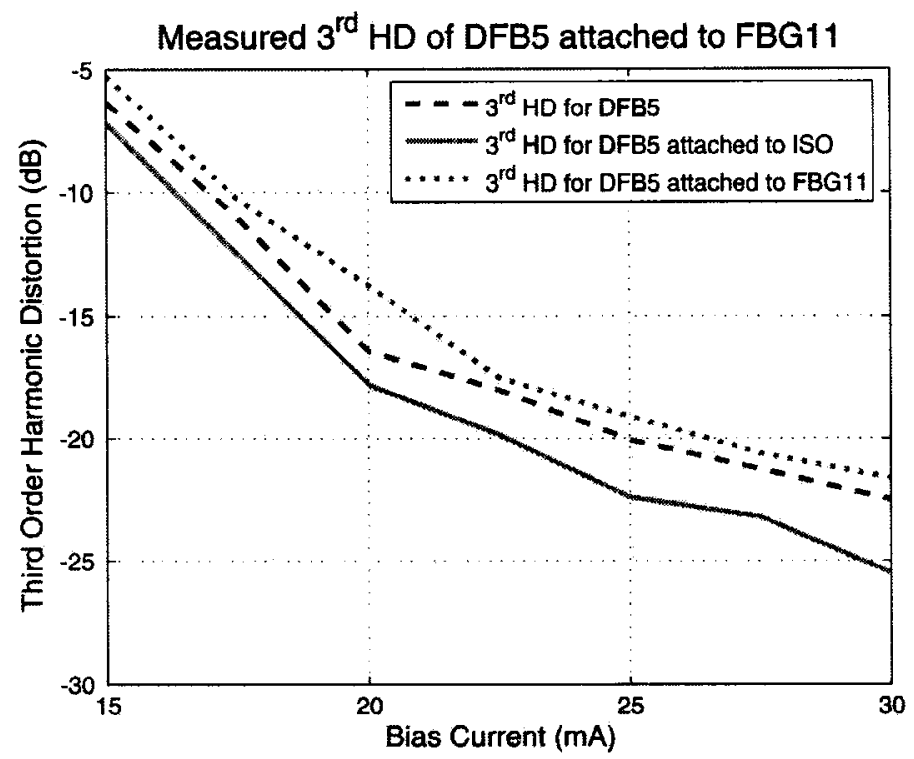

Figure B.28: Measured third order harmonic distortion normalized to the fundamental frequency of DFB5 attached to FBG11. 


\section{Bibliography}

[1] David R. Goff, Fiber Optic Video Transmission. Elsevier Science (USA), 2003.

[2] B. Buxton, and R. Vahldieck, "Noise and intermodulation distortion reduction in an optical feedforward transmitter," Microwave Symposium Digest, IEEE MTT-S International, vol. 2, pp. 1105 1108, 23-27 May 1994.

[3] F. Willens, and W. Muys, and J. Leong, "Requirements for components used in a $1550 \mathrm{~nm}$ analogue CATV system for the local loop: A view from RACE 2062 "COMFORT"," Communications, 1993. ICC 93. Geneva. Technical Program, Conference Record, IEEE International Conference on, vol. 3, pp. 1933 - 1936, 23-26 May 1993.

[4] T. E. Darcie, and G. E. Bodeep, "Lightwave subcarrier CATV transmission systems," Microwave Theory and Techniques, IEEE Transactions on, vol. 38, pp. 524 - 533, May 1990.

[5] J. van der Ziel, and R. Mikulyak, "Single-mode operation of $1.3 \mathrm{~m} \mathrm{InGaAsP} / \mathrm{InP}$ buried crescent lasers using a short external optical cavity," IEEE JQE, vol. 20, no. 3, pp. 223 - 229, 1984.

[6] L. Coldren, and T. Koch, "External-cavity laser design," Journal of Lightwave Technology, vol. LT-2, pp. 1045 - 1051, December 1984.

[7] R. S. Vodhanel, and J. S. Ko, "Reflection-induced frequency shifts in single-mode laser diodes coupled to optical fibers," Electron. Lett., vol. 20, pp. 973 -974, 8th Nov. 1984.

[8] R. Wyatt, "Spectral linewidth of external cavity semiconductor lasers with strong frequency-selective feedback," Electron. Lett., vol. 21, pp. 658 - 659, 18th July 1985.

[9] R. S. Vodhanel, and J. S. Ko, "Oscillation frequency tuning characteristics of fiber-extended-cavity distributed-feedback lasers," Appl. Phys. Lett., vol. 50, pp. $380-382$, 16th Feb. 1987.

[10] K. Petermann, Laser diode modulation and noise. Kluwer Academic Publishers, 1991.

[11] R. Tkach, and Chraplyvy, "Regimes of feedback effects in 1.5-m distributed feedback lasers," Jourmal of Lightwave Technology, vol. 4, pp. 1655 - 1661, Nov 1986.

[12] Y. Hong, S. Bandyopadhyay, S. Sivaprakasam, and et al, "Noise characteristics of a single-mode laser diode subject to strong optical feedback," Journal of Lightwave Technology, vol. 20, pp. 1847-1850, OCTOBER 2002.

[13] Gerd Keiser, Optical fiber Communications. McGraw-Hill, 2000.

[14] P. S. Andre et al, "Extraction of Laser Rate Equation Parameters," SPIE proc., vol. 3572, pp. 141-146, August 1999.

[15] D. E. Dodds, and M. J. Sieben, "Fabry-Perot Laser Diode Modeling," IEEE Phot. Tech. Lett., vol. 7, pp. 254-256, March 1995.

[16] R. Sabella, "Performance Analysis of Wireless Broadband Systems Employing Optical Fiber Links," IEEE Trans. Comm., vol. 47, pp. 715-721, May 1999. 
[17] M. Ahmed, M. Yamada, and M. Saito, "Numerical Modeling of Intensity and Phase Noise in Semiconductor Lasers," IEEE JQE, vol. 37, pp. 1600-1610, Dec. 2001.

[18] P. V. M. et al, "Rate-Equation-Based Laser Model with a Single Solution Regime," JLT, vol. 15, pp. 717-730, April 1997.

[19] S. Ghoniemy, RF/Fiber Optical Interface for Microcellular Wireless Transceivers. PhD thesis, Department of Systems and Computer Engineering, Carleton University Ottawa, Ontario, Canada, 2003.

[20] S. Ghoniemy, L. MacEachern, and S. Mahmoud, "Comprehensive Modeling of Semiconductor Lasers Including the Effect of Gain Saturation," IPR'02, July 2002.

[21] S. Ghoniemy, L. MacEachern, and S. Mahmoud, "Enhanced Large Signal Laser Modeling Including Thermal Effects for Analog Communications," IEEE LFNM'02, June 2002.

[22] S. Ghoniemy, L. MacEachern, and S. Mahmoud, "Robust Semiconductor Laser Modeling for Analog Optical Link Simulations," IEEE NUSOD-02, 2002.

[23] S. Ghoniemy, L. MacEachern, and S. Mahmoud, "Extended Robust Semiconductor Laser Modeling for Analog Optical Link Simulations," IEEE JSTQE, vol. 9, June 2003.

[24] Koji Kikushima, Osamu Hirota, Masaaki shindo, and et al, "Properties of Harmonic Distortion of Laser Diodes with Reflected Waves," Journal of Optical Communications, vol. 3, no. 4, pp. 129-132, 1982.

[25] Andreas Czylwik, "Nonlinear System Modeling of semiconductor Laser Based on Volterra Series," Journal of Optical Communications, vol. 7, no. 3, pp. 104-114, 1986.

[26] Jochen Helms, "Intermodulation and harmonic distortions of laser diodes with optical feedback," Journal of Lightwave Technology, vol. 9, pp. 1567 - 1575, Nov 1991.

[27] J. S. Cohen, R. R. Drenten, and B. H. Verbeeck, "The effect of optical feedback on the relaxation oscillation in semiconductor lasers," IEEE Journal of Quantum Electronics, vol. 24, pp. 1989 - 1995, Oct 1988.

[28] G. Agrawal, "Line narrowing in a single-mode injection laser due to external optical feedback," IEEE Journal of Quantum Electronics, vol. 20, pp. 468 - 471, May 1984.

[29] G. P. Agrawal, N. A. Olsson, and N. K. Dutta, "Effect of fiber-far-end reflections on intensity and phase noise in InGaAsP semiconductor lasers," Applied Physics Letters, vol. 45, pp. 597-599, September 1984 .

[30] N. Schunk, and K. Petermann, "Measured feedback-induced intensity noise for $1.3 \mathrm{~m}$ DFB laser diodes," Electronics Letters, vol. 25, pp. 63 - 64, Jan 1989.

[31] N. Schunk, and K. Petermann, "Numerical analysis of the feedback regimes for a single-mode semiconductor laser with external feedback," IEEE Journal of Quantum Electronics, vol. 24, pp. 1242 1247, July 1988.

[32] R. Lang, and K. Kobayashi, "External optical feedback effects on semiconductor injection laser properties," IEEE Journal of Quantum Electronics, vol. 16, pp. 347 - 355, Mar 1980.

[33] O. Hirota, and Y. Suematsu , "Quantum intensity noise of directly modulated laser diode influenced by reflected waves," Institute of Electronics and Communication Engineers of Japan, vol. E65, pp. 94101, Feb 1982.

[34] K. Peterman, and G. Arnold, "Noise and Distortion Characteristics of Semiconductor Lasers in Optical Fiber Communication Systems," IEEE Transactions on Microwave Theory and Techniques, vol. 82 , pp. $389-401$, Apr 1982. 
[35] K. Petermann, "Optical feedback phenomena in semiconductor lasers," 14 th IEEE International Semiconductor Laser Conference, pp. 8-11, Sept 1994.

[36] F. Favre, "Theoretical analysis of external optical feedback on DFB semiconductor lasers," IEEE Journal of Quantum Electronics, vol. 23, pp. 81 - 88, Jan 1987.

[37] W. I. Way, and M. M. Choy, "Optical feedback on linearity performance of 1.3-m DFB and multimode lasers under microwave intensity modulation," Journal of Lightwave technology, vol. 6, pp. 100-108, Jan 1988.

[38] J. Helms, and K. Petermann, "A simple analytic expression for the stable operation range of laser diodes with optical feedback," IEEE Journal of Quantum Electronics, vol. 26, pp. 833 - 836, May 1990.

[39] K. Petermann , "External optical feedback phenomena in semiconductor lasers," IEEE Journal of Selected Topics in Quantum Electronics, vol. 1, pp. 480 - 489, June 1995.

[40] Pan Ming-Wei, Shi Bei-Pei, and R. Gray George, "Semiconductor laser dynamics subject to strong optical feedback," Optics Letters, vol. 22, pp. 166-168, February 1997.

[41] R. J. Jones, P. S. Spencer, J. Lawrence, and D. M. Kane, "Influence of external cavity length on the coherence collapse regime in laser diodes subject to optical feedback," IEE Proceedings-Optoelectronics, vol. 148, pp. 7 - 12, Feb 2001.

[42] Ju Rui, S. Paul, S. Spencer, and K. Alan Shore, "The relative intensity noise of a semiconductor laser subject to strong coherent optical feedback ,"J. Opt. B: Quantum Semiclass. Opt., vol. 6, p. S775S779, Aug 2004.

[43] W. Bludau, and R. Rossberg, "Characterization of laser-to-fiber coupling techniques by their optical feedback," Appl. Opt., vol. 21, pp. 1933-1939, 1st June 1982.

[44] R. S. Vodhanel, and J. S. Ko, "Reflection induced frequency shifts in single-mode laser diodes coupled to optical fibers," Electron. Lett., vol. 20, pp. 973-974, 8th Nov. 1984.

[45] P. Spano, S. Piazolla, and M. Tamburrini, "Theory of noise in semiconductor lasers in the presence of optical feedback," IEEE J. Quant. Electron., vol. QE-20, pp. 350-357, Apr. 1984.

[46] N. Schunk, and K. Petermann, "Minimum bit rate of DPSK transmission for semiconductor laser with a long external cavity and strong linewidth reduction," Journal of Lightwave Technology, vol. 5, pp. 1309-1314, Sep 1987.

[47] John Rogers, and Calvin Plett, Radio Frequency Integrated Circuit Design. Artech House, Incorporated, 2003.

[48] B. Thedrez, O. Gauthier-Lafaye, F. Grillot, and et al, "1.3 $\mu \mathrm{m}$ tapered DFB lasers for isolator-free 2.5 Gbits all-optical networks," Optical Fiber Communication Conference and Exhibit 2001, vol. 3, pp. WC4-1- WC4-3, 2001.

[49] R. T. Sahara, R. A. Salvatore, A. Hohl-Abichedid, and Lu Hanh, "Isolator-free transmission at $2.5 \mathrm{Gbits} / \mathrm{s}$ over $100 \mathrm{~km}$ of single-mode fiber by a $1.55-\mathrm{m}$, AlGaInAs strained-multi-quantum-well, directly modulated distributed-feedback laser diode," IEEE Journal of Quantum Electronics, vol. 38, pp. 620-625, June 2002.

[50] W. S. Ring, I. S. Smith, A. J. Taylor, and et al , "Unisolated 2.5 Gbit/s uncooled gain-coupled 1300 nm DFB lasers for low cost applications," Electronic Components and Technology Conference 1998, pp. 192-197, 1998.

[51] K. Terada, S. Sasaki, K. Tanaka, and et al , "Isolator-free DFB-LD module with TEC control using silicon waferboard," Electrical Performance of Electronic Packaging, 1996., IEEE 5th Topical Meeting 28-30 Oct., pp. 71-73, 1996. 
[52] M. Choy, W. Yeo, T. Eustis, and et al, "High temperature and isolator-free $1310 \mathrm{~nm}$ photonic integrated surface emitter operating at $2.5 \mathrm{~Gb} / \mathrm{s}$ over $25 \mathrm{~km}$ transmission," Optical Fiber Communication Conference and Exhibit, 2002. OFC 2002 17-22 Mar 2002, pp. 719 - 721, Mar 2002.

[53] A. Mokhtar, L. MacEachern, and S. Mahmoud, "Semiconductor Laser Diode Model Implementation Including Optical Back-Rrflection," Electrical and Computer Engineering, 2006. Canadian Conference on, pp. 2146 - 2149, May 2006.

[54] A. Mokhtar, L. MacEachern, and S. Mahmoud, "Isolator-free DFB laser diode operating in feedback regime V," Proc. SPIE, Active and Passive Optical Components for Communications VI, vol. 6389, pp. $63891 \mathrm{~F}-1-63891 \mathrm{~F}-9$, Oct. 22006.

[55] K. O. Hill, and G. Meltz, "Fiber Bragg grating technology fundamentals and overview," Journal of Lightwave Technology, vol. 15, pp. 1263-1276, Aug 1997.

[56] T. Erdogan, "Fiber grating spectra," Journal of Lightwave Technology, vol. 15, pp. 1277 - 1294, Aug 1997.

[57] Andreas Othonos, and Kyriacos Kalli, Fiber Bragg gratings: fundamentals and applications in telecommunications and sensing. Artech House, 1999.

[58] B. F. Ventrudo, G. A. Rogers, G. S. Lick, and et al, "Wavelength and intensity stabilisation of $980 \mathrm{~nm}$ diode lasers coupled to fibre Bragg gratings," Electronics Letters, vol. 30, pp. 2147-2149, December 1994.

[59] T. Iwai, K. Sato, and K. I. Suto, "Signal Distortion and Noise in AM-SCM Transmission System Employing the Feedforward Linearization MQW-EW External Modulator," JLT, vol. 13, pp. $1606-$ 1612, Aug. 1995.

[60] B. Buxton, and R. Vahldieck, "Noise and Intermodulation Distortion Reduction in an optical Feedforward Transmitter," IEEE MTT-S Digest, pp. 1105 - 1108, 1994.

[61] D. Hassin, and R. Vahldieck, "Feedforward Linearization of Analog Modulated Laser Diodes: Theoretical Analysis and Experimental Verification," IEEE Trans MW Theo. and Tech., vol. 41, pp. 2376 - 2382, Dec. 1993.

[62] L. S. Fock, and R. S. Tucker, "Reduction of Distortion in Analog Modulated Semiconductor Lasers by FeedForward Compensation," Elect. Lett., vol. 27, pp. 669 -671, Apr. 1991.

[63] D. Hassin, and R. Vahldieck, "Improved Feedforward Linearization of Laser Diodes - Simulation and Experimental Results," IEEE MTT-S Digest, pp. 727 - 730, 1993.

[64] K. Tsukamoto, S. Fujii, P. Sanjo, S. Komaki, "Fiber-optic Microcellular Communication System using Frequency Modulated LD and Optical Discriminator and its Nonlinear Distortion Suppression," IEEE PIMRC'97, no. 3, pp. 984 - 988, 1997.

[65] J. Tsimbinos, and K. V. Lever, "Nonlinear Compensation using Orthogonal Inverses: Broadband Inputs and Robustness Issues," TENCON '96, vol. 2, pp. 816-821, 1996.

[66] J. Tsimbinos, and K. V. Lever, "The Computational Complexity of Nonlinear Compensators Based on the Volterra Inverse," 8th IEEE Sig. Process. Conf., pp. $387-390,1997$.

[67] H. Gysel, and M. Ramachandran, "Electrical Predistortion to Compensate for Combined Effect of Laser Chirp and Fiber Dispersion," Elect. Lett., vol. 27, pp. 421 - 423, Feb. 1991.

[68] B. Wilson, and I. Darwazeh, "Analogue Optical Fiber Communications," IEE, 1995. London.

[69] L. Roselli, and et al, "Predistortion Circuit Design for II and III Order Simultaneous Linearization in Multiservice Telecommunications Apparatuses," IEEE MTT-S Digest, pp. 1711 - 1714, 2002. 
[70] Y. Chiu, and et al, "Broadband Electronic Linearizer for Externally Modulated Analog Fiber-Optic Links," IEEE Phot. Tech. Lett., vol. 11, pp. 48 - 50, Jan. 1999.

[71] X. J. Meng, A. Yacoubian, and J. H. Bechtel, "Electro-optical Predistortion Technique for Linearization of Mach-Zehnder Modulators,"

[72] G. C. Wilson, "Optimized Predistortion of Over Modulated Mach-Zehnder Modulators with Multicarrier Input," IEEE Phot. Tech. lett., vol. 9, pp. 1535 - 1537, Nov. 1997.

[73] X. N. Fernando, and A. B. Sesay, "Higher Order Adaptive Filter Based Predistortion for Nonlinear Distortion Compensation of Radio over Fiber Links," IEEE Transactions on Electron Devices, vol. 1, pp. $367-371,2000$.

[74] M. Faulkner, and M. johansson, "Adaptive Linearization using Predistortion-Experimental Results," IEEE trans. on Veh. Tech., vol. 43, pp. $323-332$, May 1994.

[75] X. N. Fernando, and A. B. Sesay, "Look-Up Table Based Adaptive Predistortion for Dynamic Range Enhancement in a Radio over Fiber Links," IEEE Conf. on comm., comp. and SP, pp. 26 - 29, 1999.

[76] G. Steiner, S. Hunziker, and W. Baechtold, "Reduction of 3rd Order Intermodulation of a Semiconductor Laser by an Adaptive Low-Cost Predistortion Circuit at $1.8 \mathrm{GHz}$," IEEE LEOS Meeti RF Phot. for CATV and HFC Sys., July 1999.

[77] X. J. Meng, D. T. K. Tong, T. Chau, and M. C. Wu, "Demonstration of an Analog Fiber-Optic Link Employing a Directly Modulated Semiconductor Laser with External Light Injection," IEEE Phot. Tech. lett., vol. 10, pp. 1620 - 1622, Nov. 1989.

[78] D. J. M. Sabido, and et al., "Improving the Dynamic Range of a Coherent AM Analog Optical Link using Cascaded Linearized Modulator," IEEE Phot. Tech. lett., vol. 7, pp. 813 - 815, July 1995.

[79] E. D. Bedrosian, and S. O. Rice, "The output properties of volterra system (nonlinear systems with memory) driven by harmonic and gaussian inputs," Proc. IEEE, vol. 59, pp. 1688-1707, December 1971.

[80] J. J. Bussgang, and L. Ehrman, and J. W. Graham, "Analysis of nonlinear systems with multiple inputs," Proc. IEEE, vol. 62, pp. 1088-1119, August 1974.

[81] M. Schetzen, The Volterra and Wiener Theories of Nonlinear Systems. New York: John Wiley Sons, 1980. 